(C) 2009

Timothy W. Koeth

All Rights Reserved 


\title{
An Observation of a Transverse to Longitudinal Emittance Exchange at the Fermilab A0 Photoinjector \\ by Timothy W. Koeth
}

\author{
A Dissertation submitted to the \\ Graduate School-New Brunswick \\ Rutgers, The State University of New Jersey \\ in partial fulfillment of the requirements \\ for the degree of \\ Doctor of Philosophy \\ Graduate Program in Physics and Astronomy \\ written under the direction of \\ Stephen Schnetzer \\ and approved by
}

New Brunswick, New Jersey

May, 2009 


\author{
ABSTRACT OF THE DISSERTATION \\ An Observation of a Transverse to Longitudinal Emittance \\ Exchange at the Fermilab A0 Photoinjector \\ By TIMOTHY WILLIAM KOETH \\ Dissertation Director: \\ Professor Stephen Schnetzer
}

An experimental program to perform a proof of principle of transverse to longitudinal emittance exchange $\left(\epsilon_{x_{\text {in }}} \Leftrightarrow \epsilon_{z_{\text {out }}}\right.$ and $\left.\epsilon_{z_{\text {in }}} \Leftrightarrow \epsilon_{x_{\text {out }}}\right)$ has been developed at the Fermilab A0 Photoinjector. A new beamline, including two magnetic dogleg channels and a $T M_{110}$ deflecting mode radio frequency cavity, were constructed for the emittance exchange experiment.

The first priority was a measurement of the Emittance Exchange beamline transport matrix. The method of difference orbits was used to measure the transport matrix. Through varying individual beam input vector elements, such as $x_{i n}, x_{i n}^{\prime}, y_{i n}, y_{i n}^{\prime}, z_{i n}$, or $\delta_{i n}$, and measuring the changes in all of the beam output vector's elements, $x_{\text {out }}, x_{\text {out }}^{\prime}, y_{\text {out }}, y_{\text {out }}^{\prime}$, $z_{\text {out }}, \delta_{\text {out }}$, the full $6 \times 6$ transport matrix was measured. The measured emittance exchange transport matrix was in overall good agreement with our calculated transport matrix.

A direct observation of an emittance exchange was performed by measuring the electron beam's characteristics before and after the emittance exchange beamline. Operating with a 14.3 MeV, $250 \mathrm{pC}$ electron bunch, $\epsilon_{z_{i n}}$ of $21.1 \pm 1.5 \mathrm{~mm} \cdot \mathrm{mrad}$ was observed to be exchanged with $\epsilon_{x_{\text {out }}}$ of $20.8 \pm 2.00 \mathrm{~mm} \cdot \mathrm{mrad}$. Diagnostic limitations in the $\epsilon_{z_{\text {out }}}$ measurement did not account for an energy-time correlation, thus potentially returning values larger than the actual longitudinal emittance. The $\epsilon_{x_{i n}}$ of $4.67 \pm 0.22 \mathrm{~mm} \cdot \mathrm{mrad}$ was observed to be exchanged with $\epsilon_{z_{\text {out }}}$ of $7.06 \pm 0.43 \mathrm{~mm} \cdot \mathrm{mrad}$. The apparent $\epsilon_{z_{\text {out }}}$ growth is consistent with calculated values in which the correlation term is neglected. 


\section{Acknowledgements}

A project of this magnitude can only be accomplished by a small army outfitted with talented and dedicated troops. First I acknowledge the Creator whose gifts of this rich and complex universe and our ability to comprehend, be intrigued, and inquire about it all. It is my greatest honor to have been the understudy of Helen Edwards - whose advice, guidance, indefatigable energy, shared love of nature, and friendship has given me the educational experience of a lifetime. Since my first arrival at Fermilab, there has always been a motivating and comforting voice that has emanated from the shadows. This voice of reason belongs Don Edwards; I have enjoyed our numerous conversations about the present experiments as well as the many reminiscences of the golden age of accelerator physics. Leo Bellatoni, above all I thank you for your patience. My issued post-doc: Raymond P. Fliller III. Ray's ability to swing Occum's razor like a finely crafted samurai sword has trimmed my graduate student career to an minimum.

There have been several unwavering members of our team whose kindness will never go unforgotten. Jinhao Ruan, Michael Davidsaver, Jamie Santucci, Elvin Harms, Philipe Piot and Yin-E Sun. Wade Murayni, the A0 lead technician, is that technician that every other group covets! Wade's apprentice, Brad Tennis, is coming along nicely too. I appreciate the entire technical crew's assistance: Rocky, Ryan, Debbie, Jack, and Elias. A special thanks to Bruce Popper, drafter, designer, and artist; much of his art is on display on

the pages to come. I really appreciate the countless hours put in by John Reid, Rene Padilla, Tom Kubicki, Brian Chase, Julien Branlard, Gustavo Cancelo, Danny Snee, Rich Andrews, Larry Bradley, and Brian Smith. Fellow cyclotroneer, Christopher Olsen. I thank Randy Thurman-Keup, Alex Lumpkin and Vic Scarpine for lending their expertise in 'timely' measures. Amber Johnson, thank you and I wish you the best in your graduate studies. Todd \& Mary Lynn Johnson, Kermit and Caron Carlson, and Jenny Lee for your support, encouragement, and friendship. Collectively, the contribution of each of these persons efforts, professionally and personally, is what makes a nation proud of its national accelerator laboratory.

I had another incredible support team at my home institution. First, I thank my thesis advisor Steve for inviting me into his group over a decade ago, and for his support and 
encouragement to pursue this degree. Professors Chuck Joseph, Sunil Somalwar, Gordon Thomson, Tom Devlin, and Mark Croft for inspiration, guidance and education. Professor Eva Halkiadakis and Bob Stone, your friendship means the world to me. Sourabh, Shradha, and Rouven, I have immensely enjoyed being classmates and housemates and friends.

To my parents, Janet and Bill, I tell you that my success is really your success - thank you for everything! My godparents, Aunt Kathleen and Uncle Ralph's support and guidance. Aunt Joan Geigel, Aunt Lois and Uncle Charles Koeth for everything. Mom and Dad Ernst for being so supportive, encouraging, and loving to both your daughter and me throughout this process. My brothers, cohorts, cyclotroneers, and best friends: Stuart, Billy, Dan, and Jimbo; together we will always be known as the 'Mad Sci Guys.' Again, without you guys, I would not have been able to have achieved this goal. Thank you.

Finally, the two influential people to whom this thesis is dedicated. Mrs. Joanne Egli, a high school teacher of 20 years. Through QuarkNet she joined the Rutgers High Energy Physics group for the summer of 2000 in hopes of learning from us and taking fresh knowledge of High Energy Physics back to her high school classroom. After spending several weeks working side by side with me, she pulled me aside and spoke to me just as if I were a student in her classroom. Instantly I was transformed back to the tenth grade. Fiercely glaring over the rim of her glasses she said 'buster, you're just coasting - apply to graduate school now!' I promptly did. Sadly, her life was cut short. I am unable to thank Joanne for that final push, and this accomplishment is part of her legacy of as a true teacher.

My wife, Michelle Marie Koeth, an Electrical Engineer and Intellectual Property Attorney, for seeing in me what I did not see, for believing in me, understanding and supporting me. While many people played key roles in this journey, you were the one person that was there the entire time. Your love and support came in many forms: your mathematical abilities, your programming skills, your patience and impatience alike, braving four Chicago winters, providing me with bread and wine, being the 'cyclotron widow,' and finally awarding me the title of 'Physics Playboy' and all of the rights granted therein. This is ours, let the fun begin! 


\section{Dedication}

To my wife, Michelle Marie Koeth, \& to the memory of JoAnne Egli 


\section{Contents}

Abstract .............................. ii

Acknowledgements ............................ iii

Table of Contents . . . . . . . . . . . . . . . . .

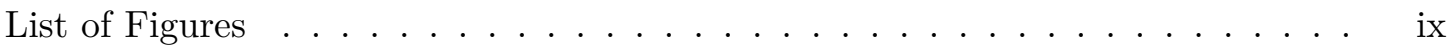

List of Tables . . . . . . . . . . . . . . . . . . . xiii

1 Introduction 1

2 Introductory Beam Physics $r 6$

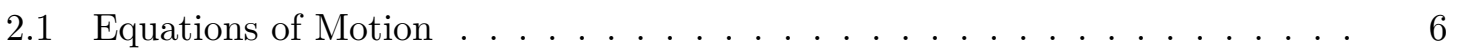

2.1 .1 Coordinates . . . . . . . . . . . . . . . 6

2.1 .2 Acceleration in $\mathrm{RF}$ fields . . . . . . . . . . . . . . . . 7

2.1.3 Transverse Motion and Transport Matrix Convention in 2D . . . . 8

2.1 .4 Closed Form Solution _ . . . . . . . . . . . . . . . . 10

2.1.5 Phase Space \& Emittance . . . . . . . . . . . . . . . . . . 11

2.1 .6 Dispersion . . . . . . . . . . . . . . . . . 14

2.1.7 Longitudinal Emittance . . . . . . . . . . . . . . . . . . . 14

2.1.8 Coupling Between Dimensions . . . . . . . . . . . . 15

2.1 .9 Momentum Compaction . . . . . . . . . . . . . . . 16

2.1 .10 Sigma Matrix . . . . . . . . . . . . . . . 17

3 Emittance Exchange Theory $\quad 19$

3.1 A Simple Approach . . . . . . . . . . . . . . . . . . . . 19

3.2 Thin vs. Thick Lens $T M_{110}$ Mode Cavity . . . . . . . . . . . . . . . . 22

3.2.1 Equations of Motion in a Pillbox Resonator . . . . . . . . . . . . 22 
3.2.2 Transfer Matrix of a Pillbox Resonator . . . . . . . . . . . . . . 24

3.3 Approximation to a Thick Lens . . . . . . . . . . . . . . 28

3.3.1 $T M_{110}$ Mode Cavity Matrix From Field Maps . . . . . . . . . . . . 28

3.4 Trace 3D Simulations . . . . . . . . . . . . . . . . . 29

3.4 .1 Trace 3D Matrix Model . . . . . . . . . . . . . . . . . . 30

3.4.2 Trace 3D Simulation for Initial Beam Conditions . . . . . . . . . . 31

4 Experimental Apparatus \& Diagnostics $\quad 36$

4.1 A0 Photoinjector Front End . . . . . . . . . . . . . . . . . . 36

4.1 .1 The Copper RF Gun . . . . . . . . . . . . . . . . 37

4.1.2 The 9-Cell Superconducting Capture Cavity . . . . . . . . . . . . 40

4.2 Integrated RF Systems . . . . . . . . . . . . . . . . . . . 42

4.2.1 $T M_{110}$ Mode Cavity High Level RF . . . . . . . . . . . . . . . 42

4.2.2 $T M_{110}$ Mode Cavity Low Level RF Control . . . . . . . . . . . . . . 44

4.2.3 $T M_{110}$ Mode Cavity Operator Interface . . . . . . . . . . . . . 49

4.3 EEX Beamline: Apparatus \& Diagnostics . . . . . . . . . . . . . . 50

4.3.1 Bunch Charge Measurement . . . . . . . . . . . . . . . . 50

4.3.2 Magnets of the Photoinjector . . . . . . . . . . . . 52

4.3 .3 Vacuum System . . . . . . . . . . . . . . 56

4.3.4 Beam Position Monitors . . . . . . . . . . . . . . . . 56

4.3.5 Intercepting Viewing Screens . . . . . . . . . . . . . . 59

4.3.6 Slit Method of Transverse Emittance . . . . . . . . . . . . . . 60

4.3.7 Bunch Length Measurements . . . . . . . . . . . . . . . . 62

5 The $T M_{110}$ Mode Cavity $\quad 71$

5.1 Cavity Basics . . . . . . . . . . . . . . . . . . . . 71

$5.2 T M_{010} \& T M_{110}$ Mode Cavity Field Descriptions $\ldots \ldots \ldots \ldots$

5.2.1 The Panofsky-Wenzel Theorem . . . . . . . . . . . . . 77

$5.3 T M_{110}$ Mode Cavity Overview and Requirements . . . . . . . . . . . 79

$5.4 T M_{110}$ Mode Cavity Construction _. . . . . . . . . . . . . . 81

$5.5 T M_{110}$ Mode Cavity Input Power Coupler . . . . . . . . . . . . . . . . . 82 
$5.6 \quad T M_{110}$ Mode Cavity Field Flatness Tuning . . . . . . . . . . . . . . . 85

$5.7 T M_{110}$ Mode Cavity Polarization . . . . . . . . . . . . . . . . . 88

$5.8 T M_{110}$ Mode Cavity Cryogenic Cooling . . . . . . . . . . . . . . . 89

$5.9 T M_{110}$ Mode Cavity First Beam . . . . . . . . . . . . . . . . . . . . 91

5.10 Beam Based Electrical Center . . . . . . . . . . . . . . . . . . . . 92

6 Measurement of EEX Transport Matrix $\quad 94$

6.1 EEX Transport Matrix Measurement Introduction . . . . . . . . . . . 94

6.2 Measurement Scheme . . . . . . . . . . . . . . . . . . . . . 95

6.3 Input Parameter Changes . . . . . . . . . . . . . . . 96

6.4 Data Collection \& Reduction . . . . . . . . . . . . . . . . . 96

6.4.1 Measuring Matrix Elements using BPMs . . . . . . . . . . . . . 96

6.4.2 Measuring Difference Orbits Using the Streak Camera . . . . . . . . 117

6.5 Survey Measurement of Columns $3 \& 4$ and Rows $3 \& 4$. . . . . . . . . . 123

6.6 Compilation of Matrix Measurements . . . . . . . . . . . . . . . . 123

7 Initial Observation of Emittance Exchange $\quad 126$

7.1 Input Beam Parameters . . . . . . . . . . . . . . . . . . 126

7.2 Input Quadrupole Scans . . . . . . . . . . . . . . . . . . 128

7.2.1 Pyroelectric-Quad Scans . . . . . . . . . . . . . . . . 128

7.3 Momentum-Quadrupole Scan . . . . . . . . . . . . . . . . . 129

7.4 Calibrated Output Projected Longitudinal Emittance . . . . . . . . . . . . 131

7.5 Observed Emittance Exchange . . . . . . . . . . . . . . . . 133

7.6 T3D MatLab Calculations . . . . . . . . . . . . . . . . . . . 134

7.7 Conclusions . . . . . . . . . . . . . . . . . . . . . . . . . . 141

8 Conclusions $\quad 142$

8.1 Emittance Exchange Future . . . . . . . . . . . . . . . . . . . . 143

8.1.1 Space Charge \& CSR Studies . . . . . . . . . . . . . . . . . 143

8.1 .2 Spatial to Temporal Modulation . . . . . . . . . . . . 145

8.1.3 'Poor Man's' Deflector . . . . . . . . . . . . . . . . . . . 145 
8.2 A $T M_{110}$ Mode Cavity for Bunch Length Measurement . . . . . . . . . . . 146

A Beam Line Element Locations

A.1 Longitudinal Element Positions w.r.t the Photocathode . . . . . . . . . . 149

B Conditioning of a TESLA Input Coupler 153

B.1 A Low Power Multipactoring Study . . . . . . . . . . . . . . . . . . 154

$\begin{array}{lll}\text { C } & T M_{110} \text { Mode Cavity Operational Notes } & 156\end{array}$

$\begin{array}{ll}\text { D Trace 3D Input File } & 158\end{array}$

Bibliography . . . . . . . . . . . . . . . . 160

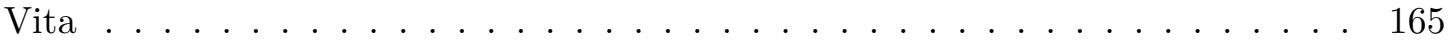




\section{List of Figures}

1.1 Looking upstream from the end of the EEX beamline . . . . . . . . . 5

2.1 Defining Edge Focusing Angles . . . . . . . . . . . . . . . . . . . . 10

2.2 Phase Space Ellipse $\ldots \ldots \ldots \ldots$. . . . . . . . . . . . . . . . . 12

2.3 Beam Envelope \& Ellipse Rotation . . . . . . . . . . . . . . . . . . . . 13

2.4 Longitudinal position change from momentum offset . . . . . . . . . . 17

3.1 Electron Trajectories through a $T M_{110}$ Cavity . . . . . . . . . . . . . 20

3.2 Conceptual EEX schematic . . . . . . . . . . . . . . . . . . . 21

3.3 Calculated EEX Matrix Turn On . . . . . . . . . . . . . . . . 32

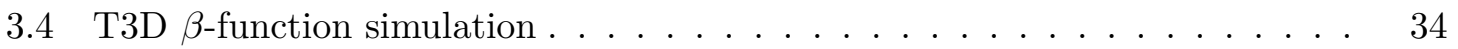

4.1 Cross Sectional View of RF Gun . . . . . . . . . . . . . . . . 38

4.2 Viewing Emittance Compensation in $x-x^{\prime}$ phase space. . . . . . . . . 39

4.3 TESLA 9-Cell Niobium Cavity . . . . . . . . . . . . . . . . . . 40

4.4 TESLA 9-Cell $R_{s}$ as a function of $T_{c} / T$, Niobium $T_{c}$ is $9.2 \mathrm{~K} \ldots \ldots$

4.5 Overview diagram of $3.9 \mathrm{GHz}$ HLRF installation. . . . . . . . . . . 43

4.6 LLRF Down Conversion Chain . . . . . . . . . . . . . . . . . . 44

4.7 Block Diagram of LLRF . . . . . . . . . . . . . . 45

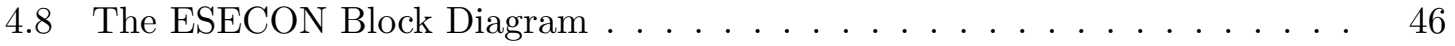

4.9 I \& Q Vector Modulation . . . . . . . . . . . . . . . . . . 48

4.10 LLRF DOOCS display . . . . . . . . . . . . . . . . . . . . . . . 49

4.11 Schematic Beamline Layout . . . . . . . . . . . . . . . 51

4.12 Installation of the Integrating Current Transformer (ICT) . . . . . . . . . 52

4.13 Pole Tip Types . . . . . . . . . . . . . . . . . . . . 53 
4.14 Pole Tip Arrangement . . . . . . . . . . . . . . . . . . . . . . 54

4.15 Magnetic Field Between Dipoles $1 \& 2 \ldots \ldots \ldots \ldots$

4.16 Schematic of a single BPM station system . . . . . . . . . . . 57

4.17 Left-Right or Up-Down BPM Button Equipotential Map . . . . . . . . . 58

4.18 Calculation of BPM Linear Response Region _ . . . . . . . . . . . . 58

4.19 Transverse Emittance Measurement _ . . . . . . . . . . . . . 61

4.20 Obtaining Beam Parameters From Slit Images _ . . . . . . . . . . . . 61

4.21 Principle of the Streak Camera Operation . . . . . . . . . . . 63

4.22 Streak Camera Image . . . . . . . . . . . . . . . . . . . 64

4.23 Comparison of streak Camera $\tau_{r e s}$ and $\tau_{b w} \ldots \ldots \ldots \ldots \ldots \ldots$

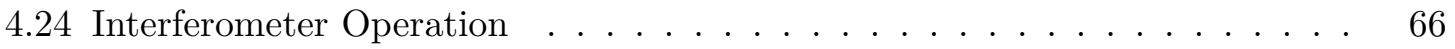

4.25 Example M-P Interferometer Analysis _ . . . . . . . . . . . . . 69

4.26 Streak Camera - Interferometer Comparison . . . . . . . . . . . . . 70

5.1 The magic of resonance $\ldots \ldots \ldots \ldots \ldots \ldots \ldots \ldots$

5.2 E and B Fields of an 'Accelerating' Cavity . . . . . . . . . . . 74

$5.3 \mathrm{E}(\mathrm{a})$ and $\mathrm{B}(\mathrm{b})$ Fields of a 'Deflecting' Cavity $\ldots \ldots \ldots \ldots \ldots \ldots$

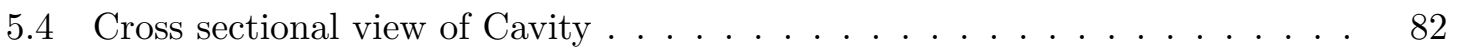

5.5 The fully brazed $T M_{110}$ Mode Cavity $\ldots \ldots \ldots \ldots$. . . . . . 83

5.6 Input Coupler $S_{21}$ Measurement $\ldots \ldots \ldots \ldots \ldots \ldots$

5.7 RF pulse $P_{f w d}, P_{r e f}$, and $P_{\text {trans }}$ signals $\ldots \ldots \ldots \ldots \ldots$

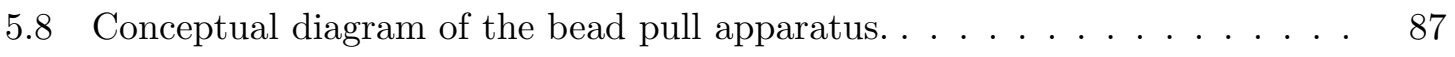

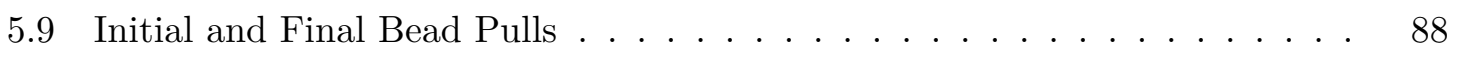

5.10 Final polarization plot $\ldots \ldots \ldots \ldots \ldots \ldots \ldots \ldots$

$5.11 T M_{110} \pi$ mode sensitivity to $l N_{2}$ bath pressure change $\ldots \ldots \ldots \ldots$.

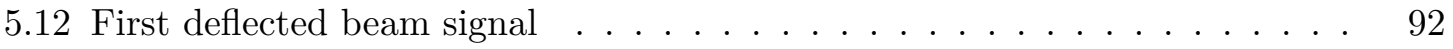

5.13 Beam based determination of $T M_{110}$ Mode Cavity electrical center. . . . . 93

6.1 BPM3 Reading During Angle Scan . . . . . . . . . . . . . . . 97

$6.2 \quad R_{12}$ Data and Analysis . . . . . . . . . . . . . . . . . . 99

6.3 Evolution of the $R_{12}$ Element $\ldots \ldots \ldots \ldots \ldots \ldots \ldots \ldots$ 
6.4 Horizontal \& vertical trajectories for differing input $\Delta x^{\prime}, k=0 \% k_{o} \ldots \ldots$

6.5 Horizontal \& vertical trajectories for differing input $\Delta x^{\prime}, k=25 \% k_{o} \ldots \ldots$

6.6 Horizontal \& vertical trajectories for differing input $\Delta x^{\prime}, k=50 \% k_{o} \ldots \ldots$

6.7 Horizontal \& vertical trajectories for differing input $\Delta x^{\prime}, k=75 \% k_{o} \ldots \ldots$

6.8 Horizontal \& vertical trajectories for differing input $\Delta x^{\prime}, k=100 \% k_{o} \ldots \ldots 107$

6.9 Comparison of two $R_{12}$ measurements f . . . . . . . . . . . . . . 108

6.10 Distribution of Input Angles for $R_{11} \ldots \ldots \ldots$

6.11 Measuring $R_{11} \ldots \ldots \ldots \ldots \ldots \ldots \ldots \ldots$

6.12 Evolution of the $R_{11}$ element $\ldots \ldots \ldots \ldots \ldots$

6.13 BPM30 Vertical Sequence . . . . . . . . . . . . . . . . . 113

$6.14 R_{66}$ Evolution . . . . . . . . . . . . . . . . 115

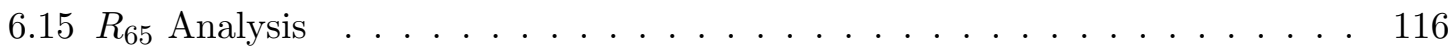

$6.16 R_{65}$ Element Evolution . . . . . . . . . . . . . . . . 117

6.17 Measuring $R_{51} \ldots \ldots \ldots \ldots \ldots \ldots \ldots \ldots \ldots$

6.18 Evolution of $R_{51} \ldots \ldots \ldots \ldots \ldots \ldots \ldots$

6.19 Measuring $R_{52}$ Element $\ldots \ldots \ldots \ldots \ldots \ldots$

6.20 Evolution of $R_{52}$ Element $\ldots \ldots \ldots \ldots \ldots$

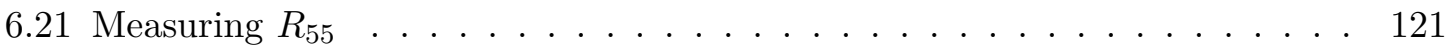

$6.22 R_{56}$ Evolution with $T M_{110}$ Mode Cavity strength . . . . . . . . . . . . . 121

6.23 Survey of vertical matrix elements . . . . . . . . . . . . . . 122

$6.244 \times 4$ EEX Matrix Measurement - Model Comparison . . . . . . . . . . . 125

7.1 Energy Spread as a Function of the 9-Cell Phase . . . . . . . . . . . . . 128

7.2 Output Bunch Length Scan . . . . . . . . . . . . . . . . . . . 129

7.3 Output Momentum Scan . . . . . . . . . . . . . . . . . . . . . 130

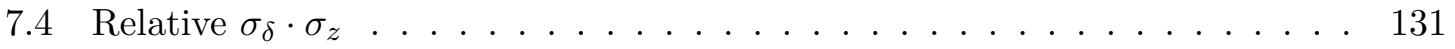

7.5 Calibrated Bunch Length and Momentum Spread Scan . . . . . . . . . . . 132

7.6 Minimum $\sigma_{\delta_{\text {out }}} \cdot \sigma_{z_{\text {out }}}$ product . . . . . . . . . . . . . 132

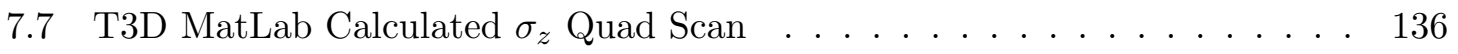

7.8 T3D MatLab Calculated $\sigma_{p}$ Quad Scan $\ldots \ldots \ldots \ldots$ 


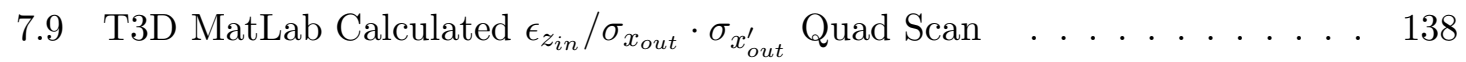

7.10 T3D MatLab Calculated $\epsilon_{x_{\text {in }}} / \sigma_{z_{\text {out }}} \cdot \sigma_{\delta_{\text {out }}}$ Quad Scan $\ldots . . . . . . .138$

7.11 T3D MatLab Calculated $\epsilon_{x_{\text {in }}} / \sigma_{z_{\text {out }}} \cdot \sigma_{\delta_{\text {out }}}$ Quad Scan for Several $\alpha_{x_{\text {in }}} \ldots \ldots$

7.12 Calculated $\epsilon_{z_{\text {in }}} / \sigma_{x_{\text {out }}} \cdot \sigma_{x_{\text {out }}^{\prime}}$ Quad Scan with Thin Lens Cavity . . . . . . 140

7.13 Calculated $\epsilon_{x_{\text {in }}} / \sigma_{z_{\text {out }}} \cdot \sigma_{\delta_{\text {out }}}$ Quad Scan with Thin Lens Cavity $\ldots . . . . .140$

8.1 Longitudinal Space Charge Field Calculation _ . . . . . . . . . . . . . 144

8.2 Using EEX for Spatial to Temporal Conversion . . . . . . . . . . . . . 146

8.3 Bunch Length Measurement With a $T M_{110}$ Mode Cavity . . . . . . . . . . 147

A.1 Schematic Beamline Layout . . . . . . . . . . . . . . . . . 152

B.1 Input Coupler Multipactoring Map . . . . . . . . . . . . . . . . . 155 


\section{List of Tables}

3.1 X3 T3D beam input parameters. . . . . . . . . . . . . . 33

4.1 A0 9-Cell and Beam Parameters . . . . . . . . . . . . . . . 37

4.2 Main dipole edge angles. . . . . . . . . . . . . . . . . . . 54

4.3 Comparison between Chromox, YAG, \& OTR Viewing Methods . . . . . . 60

4.4 Streak Camera Resolution limiting factors. . . . . . . . . . . . . . 65

$5.1 T M_{110}$ Deflecting Mode Cavity Parameters . . . . . . . . . . . . . 80

5.2 HFSS simulation to determine unity coupling . . . . . . . . . . . . . 84

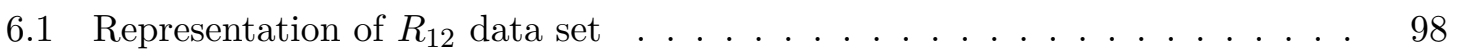

6.2 Summary of Input Angles During $R_{11}$ Measurement _ . . . . . . . . . . . 112

7.1 Summary of Feburary 62009 EEX data . . . . . . . . . . . . . . . 133

7.2 Summary of Feburary 112009 EEX data . . . . . . . . . . . . 133

A.1 Longitudinal position of beamline elements in the straight ahead beamline. 150

A.2 Longitudinal position of beamline elements in the EEX beamline. . . . . . 151 


\section{Chapter 1}

\section{Introduction}

In present day, particle accelerators have found application in fields far beyond High Energy Particle Physics. While still the principle tool for particle physicists, accelerators are used in medicine, chemistry, biological sciences, surface science, materials, engineering, and industrial processes. The requirements placed on accelerators, such as particle type, intensity, maximum energy, vary with the application, but all accelerators have in common the task of imparting energy to charged particles by applying electric and magnetic fields.

The Fermilab A0 Photoinjector is an advanced research and development electron accelerator whose focus is on the manipulation of electron beam properties. The resulting understanding and techniques will enable future accelerators to enhance their operation, reduce costly infrastructure for future installations, and even perform novel tasks not before possible.

In statistical mechanics, the state of motion of a collection of point particles may be characterized by specification of their phase space distribution. The position and motion of a particle can be described by its location and momentum vector. In the accelerator context a figure of merit of a collective group of particles (a Beam) is the phase space volume in three degrees of freedom that contains the beam. In accelerator physics the singular importance of a beam direction leads to a language resembling that of geometrical optics. The state of a particle can be referenced to that of a reference particle that follows a principal trajectory. The state of a particle's motion transversely is given by its displacement from and angle with respect to the principal trajectory. The longitudinal degree of freedom is characterized by the particles position ahead or behind the reference particle and by its momentum difference. For many accelerator applications, the tenet less is more applies for this volume. 
At a fixed energy it is convenient to speak of (unnormalized) emittance, $\epsilon_{x}, \epsilon_{y}, \epsilon_{z}$ related to the two dimensional area (position and angle) occupied by the beam particles in each degree of freedom. Under linear transformation at fixed energy, if the three degrees of freedom are uncoupled, as is usually assumed then $\epsilon_{x}, \epsilon_{y}, \epsilon_{z}$ are each conserved. If coupling does occur, then the full product is conserved. We will be dealing with a coupled system where we will be trying to show complete exchange between the $\mathrm{x}$ and $\mathrm{z}$ coordinates of phase space.

Normalized emittance, $\epsilon_{n}=\gamma \beta \epsilon$, is the term used to denote the area of a beam's phase space in each of its three degrees of freedom. Because it is directly connected with the beam momentum, the normalized emittance takes into account the apparent reduction in beam size or divergence as its momentum is changed.

Many beam applications have stringent emittance requirements for successful operation, and thus are not directly compatible with the beam characteristics of available accelerators. If the situation occurs where emittance ratios, $\epsilon_{n x}: \epsilon_{n y}: \epsilon_{n z}$, of the beam source do not match the application demands, but the total product $\epsilon_{n x} \epsilon_{n y} \epsilon_{n z}$ does, a method for transferring or exchanging the $2 \mathrm{D}$ the emittance between the three degrees of freedom is called for.

The following are just a few applications that will benefit from emittance exchange $(\mathrm{EEX}),[3]$ but as in the case of any emerging technology, the many applications only begin to appear after the announcement of a feasible method. First is a High Gain FEL, which has best performance when the transverse emittance, $\epsilon_{x}$, is less than the product of FEL wavelength, $\lambda$ and electron energy, $\gamma: \epsilon_{n x} \leq \gamma / 4 \pi$. A 1-A FEL driven by a $5-\mathrm{GeV}$ electron beam requires an transverse emittance of $\epsilon_{n x} \approx 0.1 \mathrm{~mm} \cdot \mathrm{mrad}$, an order of magnitude lower than presently achievable. State of the art Photoinjectors can produce emittances $\left(\epsilon_{n x}, \epsilon_{n y}, \epsilon_{n z}\right)$ $=(1,1,0.1) \mathrm{mm} \cdot \mathrm{mrad}$. By means of a beam manipulation technique previously pioneered at the A0 Photoinjector, called Flat Beam Transformation (FBT), the ratio of transverse emittance can be heavily coupled to produce an output emittance of $\left(\epsilon_{n x}, \epsilon_{n y}, \epsilon_{n z}\right)=(10,0.1,0.1)$ $\mathrm{mm} \cdot \mathrm{mrad}$, while still preserving the overall product.[5, 6, 7] Thus, a transverse to longitudinal emittance exchanger would complete the manipulation by performing $\epsilon_{n x} \Leftrightarrow \epsilon_{n z}$, producing the final output emittance of $\left(\epsilon_{n x}, \epsilon_{n y}, \epsilon_{n z}\right)=(0.1,0.1,10) \mathrm{mm} \cdot \mathrm{mrad}$. Current 
light source projects, such as Linac Coherent Light Source [8] and European X-FEL [9] compensate for the larger input emittances by running the driving electron beams at higher currents and greater energies; obviously emittance exchange manipulations would be beneficial.

The proposed International Linear Collider (ILC) requires small transverse emittances for high luminosity. The ILC design calls for emittances of $\left(\epsilon_{n x}, \epsilon_{n y}, \epsilon_{n z}\right)=(8,0.02,3000)$ $\mathrm{mm} \cdot \mathrm{mrad} .[10]$ The present ILC design utilizes two $6 \mathrm{~km}$ damping rings to produce the required transverse emittance. Again, combining FBT with EEX, a Photoinjector could begin with reasonable emittances $\left(\epsilon_{n x}, \epsilon_{n y}, \epsilon_{n z}\right)=(1,1,8) \mathrm{mm} \cdot \mathrm{mrad}$, and finish with $\left(\epsilon_{n x}, \epsilon_{n y}, \epsilon_{n z}\right)$ $=(8,0.02,50) \mathrm{mm} \cdot \mathrm{mrad}$, noting the significantly better $\epsilon_{n z}$ than specified.

In 2002, motivated by the obvious need for emittance exchange, P. Emma and M. Chornachia [1] proposed an ingenious arrangement of a deflecting mode radio frequency cavity within a dispersive magnetic chicane to exchange a transverse emittance, $\epsilon_{x}$, with the longitudinal emittance, $\epsilon_{z}$. In 2006 K. J. Kim and A. Sessler proposed a refinement [2] of the earlier design and is the basis of this experiment. Our goal was to demonstrate a proof of principle for a transverse-longitudinal emittance exchange, specifically $\epsilon_{x}$ with $\epsilon_{z}$. The A0 Photoinjector produces a smaller transverse emittance $\epsilon_{x, y}$ than longitudinal, $\epsilon_{z}$ (the exact ratios are dependent on experimental parameters). Thus, after undergoing the emittance exchange, we expect to see an output longitudinal emittance smaller than the output horizontal, and vice versa. For a complete exchange the ratios of $\epsilon_{x_{\text {in }}}: \epsilon_{z_{\text {out }}}$ and $\epsilon_{z_{i n}}: \epsilon_{x_{\text {out }}}$ should be 1:1. However, there are non-linear effects, such as space charge, and a non-ideal emittance exchanger that leads to some residual $x-z$ coupling and may lead to emittance dilution. It is important to note, that while our proof of principle is by exchanging input emittances that have a larger longitudinal than transverse emittance, $\epsilon_{z_{\text {in }}}>\epsilon_{x_{\text {in }}} \Rightarrow \epsilon_{x_{\text {out }}}>\epsilon_{z_{\text {out }}}$, the EEX process is linear, thus the exchange would hold for the inverse as well, $\epsilon_{x_{\text {in }}}>\epsilon_{z_{\text {in }}} \Rightarrow \epsilon_{z_{\text {out }}}>\epsilon_{x_{\text {out }}}$.

A to scale, three dimensional model of our experimental emittance exchange beamline is presented in Figure 1.1, several key components are labeled. A top view schematic of the A0 Photoinjector facility, its two beamlines, and their pertinent components are shown in Chapter 4's Figure 4.11, it is not to scale, and only indicates relative placement of 
beamline elements. The reader will find both of these figures to be an invaluable atlas to the Photoinjector throughout this document. Exact longitudinal positions of the beamline elements are available in Appendix A.

First we review the necessary beam physics in Chapter 2, upon which Chapter 3 develops the theory of emittance exchange. Chapter 4 describes the apparatus and diagnostic tools of the A0 Photoinjector and emittance exchange beamline. Chapter 5 describes the unique deflecting $T M_{110}$ Mode Cavity constructed for this experiment. After assembling and commissioning the experimental components, a measurement of the emittance exchange transfer function, e.g. the transport matrix, was completed. The transport matrix was determined by examining changes in the beam's centroid's trajectory while changing initial beam conditions and the $T M_{110}$ Mode Cavity strength and is described in Chapter 6. After a verification of the transport matrix, several direct emittance exchange measurements were taken and are reported in Chapter 7. Finally, Chapter 8 concludes this dissertation with a brief discussion on future emittance exchange studies. 


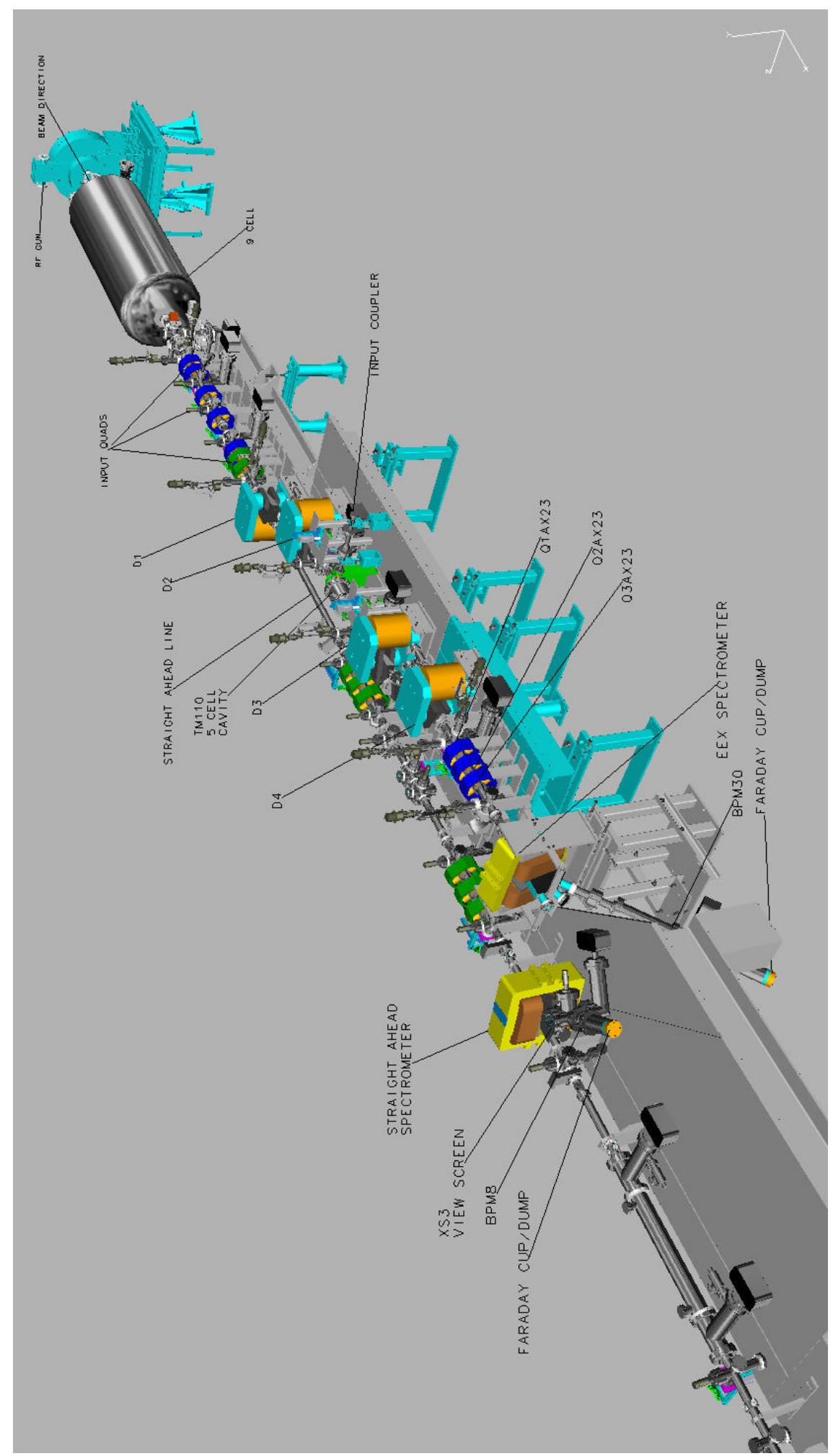

Figure 1.1: A scale model drawing of the A0 Photoinjector and Beam lines configured for the EEX experiment. The view is looking upstream from the XS4 beam dump end. (Note image is rotated $90^{\circ}$ for viewing) 


\section{Chapter 2 Introductory Beam Physics}

In this chapter we will briskly build up the relevant beam physics needed to discuss emittance exchange (EEX), we follow the treatment found in standard texts such as [11], [12], and [13].

\subsection{Equations of Motion}

The force experienced by a charged particle, $q$, traveling in electric and magnetic fields $\vec{E}$ and $\vec{B}$ is governed by the Lorentz Force:

$$
\frac{d \vec{p}}{d t}=q(\vec{E}+\vec{v} \times \vec{B})
$$

where $\vec{p}$ is the relativistic momentum and $\vec{v}$ is the velocity. Typically, but not exclusively (considering Betatrons and Induction Linacs), electric fields at radio frequencies are relegated to acceleration while magnetic fields are left the task of guiding and focusing charged particles beams.

\subsubsection{Coordinates}

Cartesian coordinates are used globally in describing the particle position and motion throughout the beamline. The principle direction of beam motion, $s$, is the longitudinal coordinate, while the two transverse directions are $x$ and $y$, horizontal and vertical respectively; together they form a right handed coordinate system. Coordinate $z$ is reserved for the local treatment of various fields, e.g. the longitudinal electric field in a radio frequency cavity or a particles longitudinal position when compared to a reference (synchronous) 
particle.

The time derivatives of a particle's position give its velocities denoted as $\left(\overrightarrow{v_{x}}, \overrightarrow{v_{y}}, \overrightarrow{v_{s}}\right)$. It's momentum, $\overrightarrow{p_{i}}$, is $\gamma m \overrightarrow{v_{i}}$, where $\gamma$ is the relativistic factor. We are operating with electrons at $14.3 \mathrm{MeV} v \approx c$, the speed of light in this experiment, hence the longitudinal velocity is generally much greater than the two transverse velocities, $v_{x}$ and $v_{y}$, such that $v_{s} \approx|v|$. The ratio of the transverse velocities with respect to the longitudinal velocities, the divergence, are denoted as $x^{\prime}=v_{x} / v_{s}$ and $y^{\prime}=v_{y} / v_{s}$; similarly the second derivatives are denoted as $x^{\prime \prime}=\partial^{2} x / \partial s^{2}$

\subsubsection{Acceleration in RF fields}

Modern day accelerators use radio frequency (RF) electric fields to work around the limitation imposed by Gauss' law [11], consequently the electric fields encountered are necessarily time dependant. Both standing wave and traveling wave resonating structures are employed as accelerating stations. In this experiment we utilize two standing wave accelerating structures, the RF Gun and the Superconducting 9-Cell, as well as a standing wave $T M_{110}$ deflecting mode cavity, known henceforth as the 5-Cell deflecting $T M_{110}$ Mode Cavity (see Chapter 5). Both the accelerating and deflecting cavities posses (harmonically) time varying longitudinal electric fields. As we will see, the magnitude of the deflecting cavity's longitudinal electric field has a transverse dependance, that is $E_{z}(x)$. For now we will consider a fixed longitudinal field, $E_{z}(z, t)$, that only varies with time and longitudinal position. Thus we can express the longitudinal electric field along the cavity axis, $z$, as:

$$
E_{z}(z, t)=E_{o}(z) \cos (\omega t+\phi)
$$

All of the A0 Photoinjector cavities are standing wave, multi-cell resonators operating in the so called $\pi$-mode. That is, the instantaneous phase of the Electric and Magnetic fields are $\pi$-radians $\left(180^{\circ}\right)$ advanced between cells. Since the electrons we will encounter are considered to have $v_{s} \approx c$, the physical length of each cell is manufactured to be $\lambda / 2$ at the operating frequency, thus a $v_{s} \approx c$ particle repeatedly experiences the same electric and magnetic field in each cell modulo $2 \pi$. Thus, we can consider a single cell and apply the 
behavior to an $N$-cell structure. Further, we introduce the synchronous reference particle, the particle that crosses the cavity's center which we now define as $z=0$ at the peak field time $t=0$, equivalently when $\phi=0$. It stands to reason, that a non-synchronous enters the cavity some time before or after the synchronous particle, that is $\phi \neq 0$.

$$
\frac{d p_{z}}{d t}=q E(z) \cos (\omega t+\phi)
$$

Next we must define the transit time factor. Even the synchronous particle only encounters the peak $E_{z}(z, t)$ at $z=0$, otherwise the electric field is ascending to or descending from crest. Thus the synchronous particle just enters the $\lambda / 2$ cell at $E_{z}(z=-\lambda / 4, t) \mid t=-\pi / 2$, thus experiencing no electric field. Thus we can solve for the overall reduction in integrated electric field, a constant known as the transit time factor $T$ :

$$
T=\frac{\int_{-\lambda / 4}^{\lambda / 4} E_{z}(z) \cos \left(\frac{2 \pi}{\lambda} z\right) d z}{\int_{-\lambda / 4}^{\lambda / 4} E_{z}(z) d z}
$$

which, for a uniform $E_{z}(z), \pi$-mode structure is $2 / \pi$. The integrated electric field experienced by an electron crossing the cavity with $\phi$ offset from the synchronous particle is:

$$
V_{o}=\frac{2}{\pi} \cos (\phi) \int_{-\lambda / 4}^{\lambda / 4} E_{z} d z
$$

Thus the synchronous particle, $\phi=0$, is awarded the maximum energy gain.

\subsubsection{Transverse Motion and Transport Matrix Convention in 2D}

Taking $u$ to stand for either $x$ or $y$ and $K(s)$ as the focusing function we write the equation of motion in the form of Hill's equation:[11]

$$
u^{\prime \prime}+K(s) u=0
$$

Equation 2.6 is a second order differential equation which lacks a first derivative term indicating that no dissipative or driving force is encountered. Thus Equation 2.6 is that of a harmonic oscillator except the that restoring force varies longitudinally. Taking $K(s)$ 
$=\kappa$, a constant for a distance $l$, over the interval $s_{i n}<s<s_{\text {out }}$, a piecewise solution to Equation 2.6 can be written in matrix form as:

$$
\left(\begin{array}{c}
u \\
u^{\prime}
\end{array}\right)_{\text {out }}=\left(\begin{array}{ll}
R_{11} & R_{12} \\
R_{21} & R_{22}
\end{array}\right)\left(\begin{array}{c}
u \\
u^{\prime}
\end{array}\right)_{\text {in }}
$$

Where $u_{i n}$ is the initial transverse displacement and $u_{i n}^{\prime}$ is the initial divergence. Units of $m m$ and $m r a d s$, respectively, are used throughout this document. The $2 \times 2$ matrix is known as the the transfer matrix for one degree of freedom, condensing the notation, the 2 $\times 2$ matrix is represented by just $R$.

$$
\left(\begin{array}{c}
u \\
u^{\prime}
\end{array}\right)_{\text {out }}=R\left(\begin{array}{c}
u \\
u^{\prime}
\end{array}\right)_{\text {in }}
$$

Solving Equation 2.7 three interesting scenarios arise: first, where $\kappa<0$, secondly, where $\kappa>0$, and finally, $\kappa=0$ where no focusing force is experienced.

For $\kappa<0$ the results is of a defocusing element:

$$
R=\left(\begin{array}{cc}
\cosh \sqrt{|\kappa| l} l & \frac{\sin \sqrt{|\kappa|} l}{\sqrt{|\kappa|}} \\
\sqrt{|\kappa|} \sin \sqrt{|\kappa|} l & \cos \sqrt{|\kappa|} l
\end{array}\right)
$$

For $\kappa>0$ the result is of a focusing element:

$$
R=\left(\begin{array}{cc}
\cos \sqrt{\kappa} l & \frac{\sin \sqrt{\kappa} l}{\sqrt{\kappa}} \\
-\sqrt{\kappa} \sin \sqrt{\kappa} l & \cos \sqrt{\kappa} l
\end{array}\right)
$$

Finally, for $\kappa=0$ the result is a drift transport matrix of length $l$ :

$$
R=\left(\begin{array}{ll}
1 & l \\
0 & 1
\end{array}\right)
$$

An assembly of many sub-focusing, defocusing, and drifts elements can be described by a single beam transport matrix. These transport matrices are Jacobian matrices, and in the accelerator physics community, are typically referred to as ' $R$ ' matrices. Matrix 
multiplication of each beamline element in the order in which they appear will yield a final transport matrix, i.e. if the elements are experienced in order of $1,2, \ldots, n$, then the final $R$ matrix is $R=R_{n} \times \ldots \times R_{2} \times R_{1}$.

This matrix notation is not confined to focusing elements, other beam line elements are similarly described. For example, the transport matrix of a dipole wedge magnet of bending radius $\rho$ and bending angle $\theta$ is written as:

$$
R=\left(\begin{array}{cc}
\cos \theta & \rho \sin \theta \\
-\frac{\sin \theta}{\rho} & \cos \theta
\end{array}\right)
$$

Dipole magnets whose entrance and exit pole tip edges are not normal to the respective incident and exiting beam trajectories develop focusing terms, inevitably named edgefocusing. The transport matrix in the bend plane of an edge focusing dipole can be disassembled into three matrices, edge-dipole-edge, where $\eta_{\text {enter }}$ and $\eta_{\text {exit }}$ are the respective entrance and exit angles and are depicted in Figure 2.1. In Figure 2.1 $\eta_{\text {enter }}$ and $\eta_{\text {exit }}$ are both defined as positive.

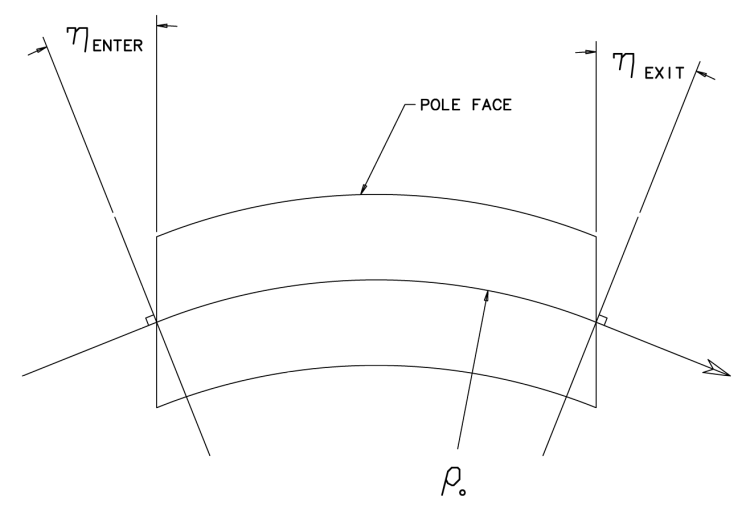

Figure 2.1: Convention used to define edge angles.

$$
R=\left(\begin{array}{cc}
1 & 0 \\
-\frac{\tan \eta_{\text {exit }}}{\rho} & 1
\end{array}\right)\left(\begin{array}{cc}
\cos \theta & \rho \sin \theta \\
-\frac{\sin \theta}{\rho} & \cos \theta
\end{array}\right)\left(\begin{array}{cc}
1 & 0 \\
-\frac{\tan \eta_{\text {enter }}}{\rho} & 1
\end{array}\right)
$$

\subsubsection{Closed Form Solution}

A general solution to 2.6 can be written as: 


$$
u(s)=J \sqrt{\beta_{u}(s)} \cos \left(\psi_{u}(s)+\psi_{o}\right)
$$

Where $J$ is a constant, $\beta(s)$ is the so-called the amplitude function (also referred to as the beta function or $\beta$-function) and $\psi_{u}(s)$ is the phase advance which is calculated by:

$$
\psi(s)=\int_{0}^{s} \frac{d s^{\prime}}{\beta\left(s^{\prime}\right)}
$$

At this point, we introduce two more parameters that relate to the amplitude function

$$
\begin{aligned}
& \alpha(s)=-\frac{1}{2} \frac{d \beta(s)}{d s} \\
& \gamma(s)=\frac{1+\alpha(s)^{2}}{\beta(s)}
\end{aligned}
$$

After taking the appropriate derivatives of 2.14 and substitutions of 2.16 and 2.17 we can write

$$
\alpha(s) u(s)+\beta(s) u^{\prime}(s)=-J \sqrt{\beta_{u}(s)} \sin \left(\psi_{u}(s)+\psi_{o}\right)
$$

Squaring to ablate the trigonometric function and re-arranging, we arrive at the equation of an ellipse:

$$
J^{2}=\gamma(s) u(s)^{2}+2 \alpha(s) u(s) u^{\prime}(s)+\beta(s) u^{\prime}(s)^{2}=\epsilon
$$

The ellipse parameters $\alpha, \beta$, and $\gamma$, are known as the Courant-Synder parameters and describe the shape and rotation of the ellipse.[14]

\subsubsection{Phase Space \& Emittance}

We now write the emittance in terms of the $2 \mathrm{D}$ ellipse's area, A, as:

$$
\epsilon=\frac{A}{\pi}=\gamma(s) u(s)^{2}+2 \alpha u(s) u(s) u^{\prime}(s)+\beta(s) u^{\prime}(s)^{2}
$$




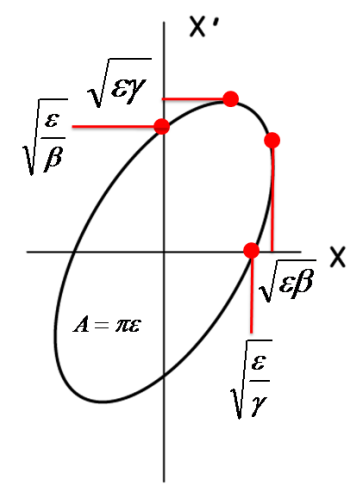

Figure 2.2: A phase space diagram in the $x-x^{\prime}$ plane.

First, we consider a single particle trajectory through a beamline transport. In a repetitive transport lattice, such as a synchrotron, a particle that returns to the same location on successive turns will experience the same $\alpha, \beta$, and $\gamma$ parameters and will lie on the same ellipse but at a different phase. After many turns, the particle will trace out the ellipse for that location. At different locations, the $\alpha, \beta$, and $\gamma$ will be different but the emittance related to that particle will be the same. Thus the ellipse will have a different shape but the same area. The as the particle propagates in $z$ (either in a beamline or in a circular accelerator) it traces out a point in $x-x^{\prime}$ space known as trace space. Thus the beam, an ensemble of particles, is a collection of nested trace space ellipses.

Next, we assume a beam composed of a Gaussian particle distribution in each degree of freedom. Liouville's Theorem states that for non-interactive particles, the six-dimensional volume they occupy is invariant.[11] In practice the 2-D projections of each coordinate phase space pairs, i.e. $x-x^{\prime}, y-y^{\prime}, z-\delta$ describe the ellipse on which they lie. It is then convenient to identify a representative phase space ellipse that bounds a standard fraction of the beam, such as drawn in Figure 2.2. Taking that boundary to contain one standard deviation of the particles, we can, for example, write the phase space occupation in terms of the horizontal unnormalized root mean square (rms) beam emittance as:

$$
\epsilon_{x}=\sqrt{\sigma_{x}^{2} \sigma_{x^{\prime}}^{2}-\left(\sigma_{x x^{\prime}}\right)^{2}}
$$

Where $\sigma_{x}$ and $\sigma_{x^{\prime}}$ are the horizontal $\mathrm{rms}$ beam size and divergence respectively; $\sigma_{x x^{\prime}}$ is the linear correlation between position and divergence for an ensemble of particles, where 


$$
\sigma_{x x^{\prime}}=\frac{1}{N} \sum_{k=1}^{N} x_{k} x_{k}^{\prime}
$$

Figure 2.3 depicts the collective contribution of many individual trajectories to a beam envelope. The transverse rms beam sizes are determined from the amplitude function and emittance by Equation 2.23.

$$
\sigma_{u}=\sqrt{\epsilon_{u} \beta_{u}}
$$

Further, the transverse rms beam divergence can be calculated from the emittance times the Courant-Synder $\gamma$ :

$$
\sigma_{u^{\prime}}=\sqrt{\epsilon_{u} \gamma_{u}}
$$

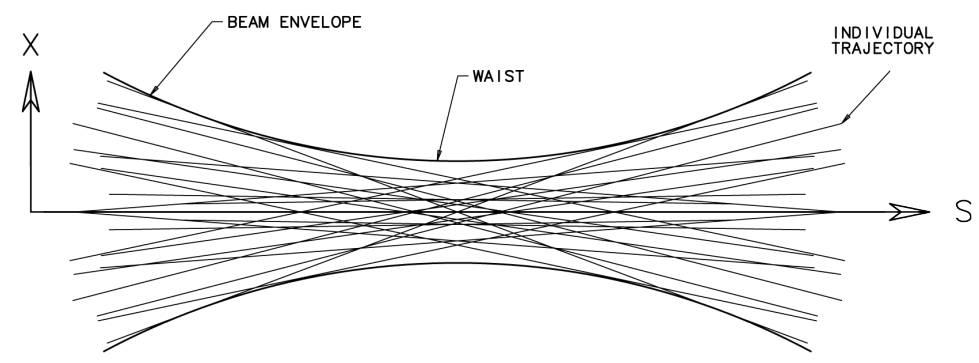

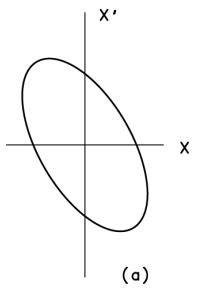

CONVERGENT

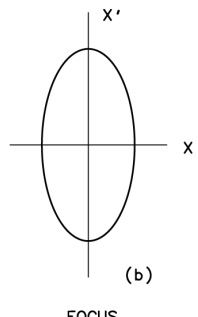

Focus

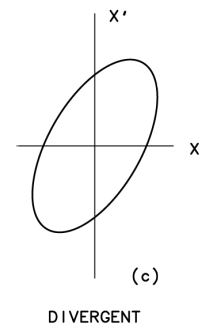

DIVERGENT

Figure 2.3: An illustration of individual particle trajectories which comprise a beam propagating in a drift. Corresponding ellipses are drawn at three interesting phases of the evolution: the beam enters as a convergent beam (a), reaches a focus (b), and again becomes divergent (c). Note that while the ellipse rotates, it's area remains constant.

Normalized emittance, $\epsilon_{n x}$ allows for comparison of the beam at differing momenta, proportional to relativistic $\beta \gamma$, and thus is calculated from $\epsilon_{x}$ by:

$$
\epsilon_{n x}=\beta \gamma \epsilon_{x}
$$


Again, carefully noting that $\beta=v / c$ and $\gamma$ are the relativistic factors.

When the degrees of freedom $\left(x-x^{\prime}, y-y^{\prime}, z-\delta\right)$ are uncoupled, the normalized emittances $\epsilon_{n x}, \epsilon_{n y}, \epsilon_{n z}$ are each conserved. More generally, when coupling between planes occurs the full six dimensional (6D) normalized emittance, $\epsilon_{n 6 D}=\epsilon_{n x} \epsilon_{n y} \epsilon_{n z}$, is conserved.

\subsubsection{Dispersion}

In a beam, a particle that deviates from the central momentum by $\delta \equiv \frac{\Delta p}{p_{o}}$ will suffer a chromatic error when deflected, for instance, by a dipole magnet of bending radius $\rho_{o}$. Assuming $\Delta p \ll p_{o}$ we can write 2.6 with the first order perturbation:

$$
u^{\prime \prime}+K(s) u=\frac{1}{\rho_{o}}(s) \delta
$$

A general solution to 3.46 is:

$$
\left(\begin{array}{c}
u \\
u^{\prime} \\
\delta
\end{array}\right)_{\text {out }}=\left(\begin{array}{ccc}
R_{11} & R_{12} & D \\
R_{21} & R_{22} & D^{\prime} \\
0 & 0 & 1
\end{array}\right)\left(\begin{array}{l}
u \\
u^{\prime} \\
\delta
\end{array}\right)_{\text {in }}
$$

In the case of a horizontal bending magnet, the excursion from the ideal trajectory is now the superposition of the displacement resulting from the transfer function, $R$, and the dispersion function. It is useful to outline that in the case of a horizontal bending magnet the horizontal rms spot size is the quadrature sum of the statistical contributions from the amplitude and dispersion functions.

$$
\sigma_{x}^{2}=\epsilon_{x} \beta_{x}+D_{x}^{2}\left\langle\left(\frac{\Delta p}{p_{o}}\right)^{2}\right\rangle
$$

\subsubsection{Longitudinal Emittance}

A transverse to longitudinal emittance exchange requires a prescription for relating the two dimensions. We assume the longitudinal phase space can be approximated by an ellipse. The longitudinal rms bunch length is reported in millimeters, relates to its longitudinal extent by $z \approx c t$ and the rms fractional momentum spread, reported in parts per mil (thus 
being equivalent to $x^{\prime}$ units of milliradians): $\frac{\sigma_{p}}{\langle p\rangle} \equiv \delta$, facilitating a direct comparison. Thus, the un-normalized longitudinal emittance is

$$
\epsilon_{z}=\sqrt{\sigma_{z}^{2} \sigma_{\delta}^{2}-\left(\sigma_{z \delta}\right)^{2}}
$$

The beam chirp, $\sigma_{z \delta}$, is the linear change in energy along the bunch length. Just as in the transverse case, the normalized longitudinal emittance is arrived at by multiplying $\epsilon_{z}$ by the relativistic factors $\beta$ and $\gamma$ :

$$
\epsilon_{n z}=\beta \gamma \epsilon_{z}
$$

Similarly to 2.23 the longitudinal $\mathrm{rms}$ bunch length is related to the longitudinal parameters in the approximation that the longitudinal phase space is defined by an ellipse:

$$
\sigma_{z}=\sqrt{\epsilon_{z} \beta_{z}}
$$

Equivalent to the $2 \mathrm{D}$ transverse phase space beam divergence, the longitudinal fractional momentum spread, $\delta$, can be determine from:

$$
\sigma_{\delta}=\sqrt{\epsilon_{z} \gamma_{z}}
$$

where $\gamma$ is the Courant-Synder parameter.

\subsubsection{Coupling Between Dimensions}

So far only stwo-dimensional transport matrices have been considered. The extension to $n$-dimensions is natural and necessary when considering coupling between planes. Shown below in Equation 2.33 is a typical $4 \times 4 R$ transport matrix describing both the transverse horizontal and longitudinal dimensions. 


$$
\left(\begin{array}{l}
x \\
x^{\prime} \\
z \\
\delta
\end{array}\right)_{\text {out }}=\left(\begin{array}{llll}
a_{11} & a_{12} & b_{11} & b_{12} \\
a_{21} & a_{22} & b_{21} & b_{22} \\
c_{11} & c_{12} & d_{11} & d_{12} \\
c_{21} & c_{22} & d_{21} & d_{22}
\end{array}\right)\left(\begin{array}{l}
x \\
x^{\prime} \\
z \\
\delta
\end{array}\right)_{\text {in }}
$$

The $\mathbf{A}$ on-diagonal sub block relates the $x_{\text {input }}$-plane parameters contribution to the $x_{\text {out }}{ }^{-}$ plane parameters, similarly the $\mathbf{D}$ relates the input and output $z$-plane parameters. The $\mathbf{B}$ and $\mathbf{C}$ off-diagonal sub blocks describe the $x$ and $z$ inter-plane coupling terms.

We will develop the transport matrix of an emittance exchange beamline whose coupling terms are dominant, that is $\mathbf{B}$ and $\mathbf{C}$ sub-block elements are large compared with $\mathbf{A}$ and D, in Chapter 3

\subsubsection{Momentum Compaction}

A dispersive magnetic channel results in energy-dependent trajectories with differing longitudinal path lengths. This feature can be exploited to generate short bunches by what is known as 'bunch compression.' Consider an electron bunch with a linear energy-time correlation traveling through a magnetic dogleg as depicted in Figure 2.4. Assume the head of the electron bunch has a lower momentum than the bunch tail. It arrives at the first dipole and will be bent harder, taking a longer path. Conversely, the later arriving tail has a higher momentum, thus deflects less through the first magnet and takes a shorter longitudinal path. The longitudinal location of maximum compression can be controlled by tuning the incoming energy-time correlation (chirp). Over-compression occurs when the tail of the bunch longitudinally overtakes the head, resulting in a longitudinal bunch which is longer than the bunch at minimum compression as the tail is now in front of the head.

Now consider an electron that has passed through an accelerating RF cavity and enters the magnetic dogleg with coordinates $\left(s_{i n}, \delta_{i n}\right)$, where $\delta$ is the fractional momentum offset from the synchronous particle. If the bunch length is short compared to the accelerating RF wavelength, $\sigma_{z}<\lambda / 2 \pi$, a linear approximation of the energy-time chirp, $\sigma_{z \delta, i n}$ is justified, and it is satisfactory to describe the longitudinal progression $s_{\text {out }}$ of the non-synchronous electron as: 


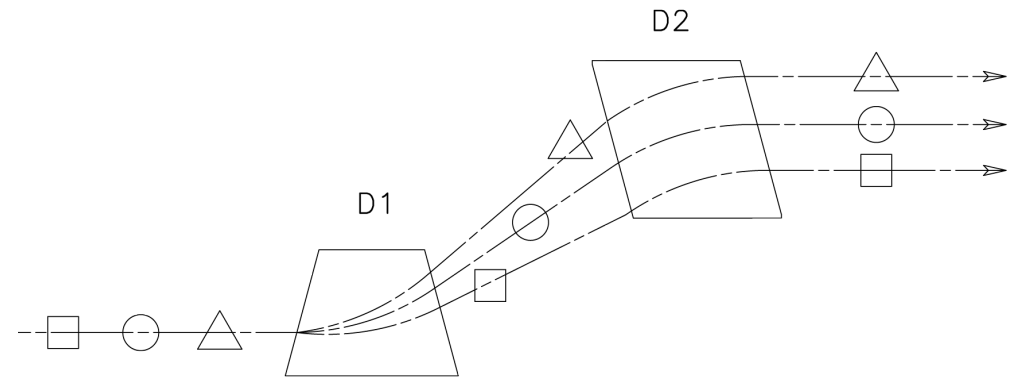

Figure 2.4: A conceptual drawing of momentum compaction. Consider a reference particle, the circle, of the nominal momentum. If the head of the bunch (triangle) has a slightly lower momentum, it bends more taking a longer path, conversely, if the tail of the bunch (square) has a slightly higher momentum it bends less taking a shorter path. Eventually particles of all momenta longitudinally align.

$$
z_{\text {out }}=z_{\text {in }}+R_{56} \delta_{\text {in }}
$$

Where $R_{56} \equiv \partial z_{\text {out }} / \partial \delta_{\text {in }}$ is the so-called momentum compaction factor of the transport matrix. The rms bunch length can be written as:

$$
\sigma_{z, \text { out }}=\sqrt{\left(1+\sigma_{z \delta, \text { in }} R_{56}\right)^{2} \sigma_{z, \text { in }}^{2}+R_{56}^{2} \sigma_{\delta}^{2}}
$$

\subsubsection{Sigma Matrix}

We re-write Equation 2.20 in sigma notation as well as drop the explicit $(s)$ dependence though it is still implied.[34]

$$
\sigma_{22} x^{2}-2 \sigma_{12} x x^{\prime}+\sigma_{11} x^{\prime 2}=\epsilon^{2}
$$

Again, assuming the longitudinal phase space is approximated by an ellipse, an equivalent expression for the $z$ plane can be written with $z$ in place of $x$ in 2.36. A convenient form to write the collective beam parameters, $\epsilon, \alpha, \beta$, and $\gamma$, is in the sigma matrix convention. Below they are related to the now familiar Courant-Snyder parameters as:

$$
\sigma=\left(\begin{array}{ll}
\sigma_{11} & \sigma_{12} \\
\sigma_{21} & \sigma_{22}
\end{array}\right)=\left(\begin{array}{cc}
\epsilon \beta & -\epsilon \alpha \\
-\epsilon \alpha & \epsilon \gamma
\end{array}\right)
$$


The projected emittance can readily be solved for by taking the square root of the determinant of $\sigma$ :

$$
\epsilon_{r m s}=\sqrt{\operatorname{det} \sigma}
$$

In the $4 \mathrm{D}$ treatment the collective beam matrix is [17]

$$
\sigma=\left(\begin{array}{cc}
\sigma_{x} & 0 \\
0 & \sigma_{z}
\end{array}\right)=\left(\begin{array}{cccc}
\epsilon_{x_{o}} \beta_{x_{o}} & -\epsilon_{x_{o}} \alpha_{x_{o}} & 0 & 0 \\
-\epsilon_{x_{o}} \alpha_{x_{o}} & \epsilon_{x_{o}} \gamma_{x_{o}} & 0 & 0 \\
0 & 0 & \epsilon_{z_{o}} \beta_{z_{o}} & -\epsilon_{z_{o}} \alpha_{z_{o}} \\
0 & 0 & -\epsilon_{z_{o}} \alpha_{z_{o}} & \epsilon_{z_{o}} \gamma_{z_{o}}
\end{array}\right)
$$

Recalling the $4 \times 4$ beamline transport matrix $R$,

$$
R=\left(\begin{array}{ll}
A & B \\
C & D
\end{array}\right)=\left(\begin{array}{cccc}
a_{11} & a_{12} & b_{11} & b_{12} \\
a_{21} & a_{22} & b_{21} & b_{22} \\
c_{11} & c_{12} & d_{11} & d_{12} \\
c_{21} & c_{22} & d_{21} & d_{22}
\end{array}\right)
$$

The benefit of the sigma matrix notation is revealed by the simplicity of transporting the beam matrix, $\sigma$, through the transport $R$ relating two positions, $s_{\text {in }}$ and $s_{\text {out }}$ :

$$
\sigma_{\text {out }}=R \sigma_{\text {in }} R^{T}
$$

where $R^{T}$ is the transpose of matrix $R$. Propagating the initial sigma matrix of Equation 2.39 the $R$ matrix of Equation 2.40 the resulting output sigma matrix reads:

$$
\sigma_{\text {out }}=\left(\begin{array}{cc}
A \sigma_{x} A^{T}+B \sigma_{z} B^{T} & A \sigma_{x} C^{T}+B \sigma_{z} D^{T} \\
C \sigma_{x} A^{T}+D \sigma_{z} B^{T} & C \sigma_{x} C^{T}+D \sigma_{z} D^{T}
\end{array}\right)
$$

It is with this equation that we will calculate the ratio of emittance exchange in Chapter 7. 


\section{Chapter 3}

\section{Emittance Exchange Theory}

Using the beam physics tools covered in Chapter 2, we endeavor to develop a beamline that can produce an emittance exchange (EEX) between the horizontal and longitudinal planes. We follow the design of K.-J. Kim, [2] however other EEX beamline arrangements exist, the generalized requirements of an EEX beamline were reviewed by R. Filler.[15]. We see that complete emittance exchange would occur for a beam transport matrix where the on-diagonal sub-blocks elements, $A$ and $D$, are zero, such as the following:

$$
R=\left(\begin{array}{cc}
A & B \\
C & D
\end{array}\right)=M_{E E X}=\left(\begin{array}{cccc}
0 & 0 & b_{11} & b_{12} \\
0 & 0 & b_{21} & b_{22} \\
c_{11} & c_{12} & 0 & 0 \\
c_{21} & c_{22} & 0 & 0
\end{array}\right)
$$

In this chapter we first develop a thin lens model beamline which creates an exact exchange beamline. A thick lens cavity approximation follows, with discussion of the resulting non-zero on-diagonal sub-block elements.

\subsection{A Simple Approach}

We first begin with a simplified presentation which is remarkably accurate. We use thin lens approximations for the $T M_{110}$ Mode Cavity and bending dipoles and assume the electron bunch length is much shorter than the effective RF wavelength, $\sigma_{z} \ll \lambda_{R F}$. We set the phase of $T M_{110}$ Mode Cavity such that the synchronous particle crosses the center of the cavity, $z=0$, at the peak electric field (zero B-field crossing), i.e. $t=0, \phi=0$. The full development of the $T M_{110}$ Mode Cavity fields is performed in Chapter 5. For now it suffices 


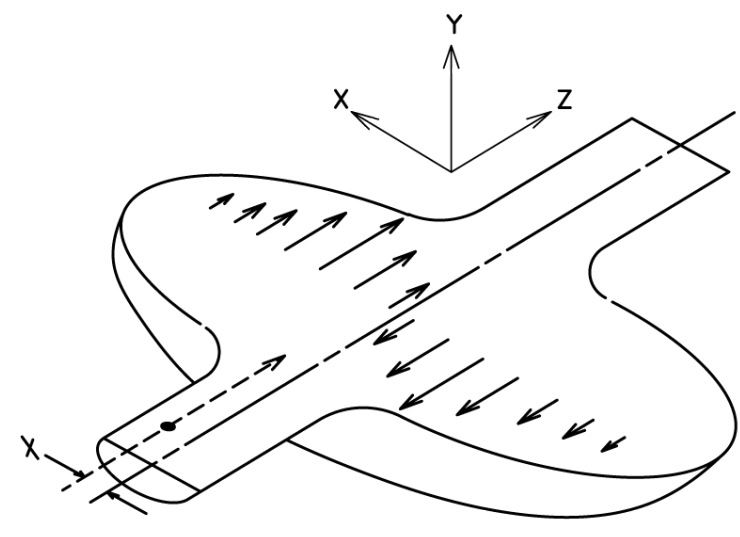

(a)

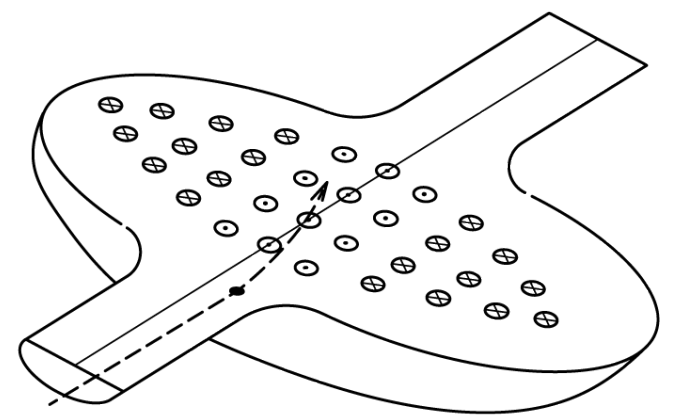

(b)

Figure 3.1: Trajectories of an electron at different phases through the $T M_{110}$ Mode Cavity . (a) depicts the peak $E_{z}$ field in the horizontal plane, while (b) depicts the peak vertical magnetic field which occurs $90^{\circ}$ later in time.

to state that, as depicted in Figure 3.1(a), an electron traveling through the cavity with a horizontal offset $x$ displaced from the horizontal axis will experience a fractional energy change $\delta$ :

$$
\delta \approx \frac{e V_{o}}{E \rho} x=k x
$$

where $V_{o}$ is the potential experienced by a electron of energy $E$ traveling through the cavity at a horizontal displacement $x$ from the axis, and $\rho$ is a constant characteristic of the cavity's geometry. We then define $k$ as the cavity strength, is:

$$
k \equiv \frac{e V_{o}}{E \rho}
$$

Additionally, a non synchronous particle (one that arrives at the center of the cavity before or after the reference particle, peak electric field, i.e. $\phi=0)$ will experience a transverse deflection $\Delta x^{\prime}$ from the non-zero $(\phi \neq 0)$ vertical magnetic field depicted in Figure 3.1(b):

$$
\Delta x^{\prime} \approx \frac{e V_{o}}{E \rho} \Delta z=k \Delta z,
$$

Thus, the thin lens cavity matrix is; 


$$
M_{\text {cavity }}=\left(\begin{array}{cccc}
1 & 0 & 0 & 0 \\
0 & 1 & k & 0 \\
0 & 0 & 1 & 0 \\
k & 0 & 0 & 1
\end{array}\right)
$$

The matrix of a magnetic dogleg is

$$
M_{d}=\left(\begin{array}{cccc}
1 & L & 0 & \alpha L \\
0 & 1 & 0 & 0 \\
0 & \alpha L & 1 & \alpha^{2} L \\
0 & 0 & 0 & 1
\end{array}\right)=\left(\begin{array}{cccc}
1 & \frac{D}{\alpha} & 0 & D \\
0 & 1 & 0 & 0 \\
0 & D & 1 & \alpha D \\
0 & 0 & 0 & 1
\end{array}\right)
$$

where $L$ is the distance between the two thin lens bends of the dogleg, and $\alpha$ is the bending angle. The second matrix of equation 3.6 is written in terms of the dispersion $D=\alpha L$.

Now we construct an emittance exchanger by placing the thin lens $T M_{110}$ Mode Cavity centered in a drift of length $2 l$ between the two identical (not a necessary condition) doglegs as diagrammatically shown in Figure 3.2. The resulting emittance exchange transport matrix is written as:

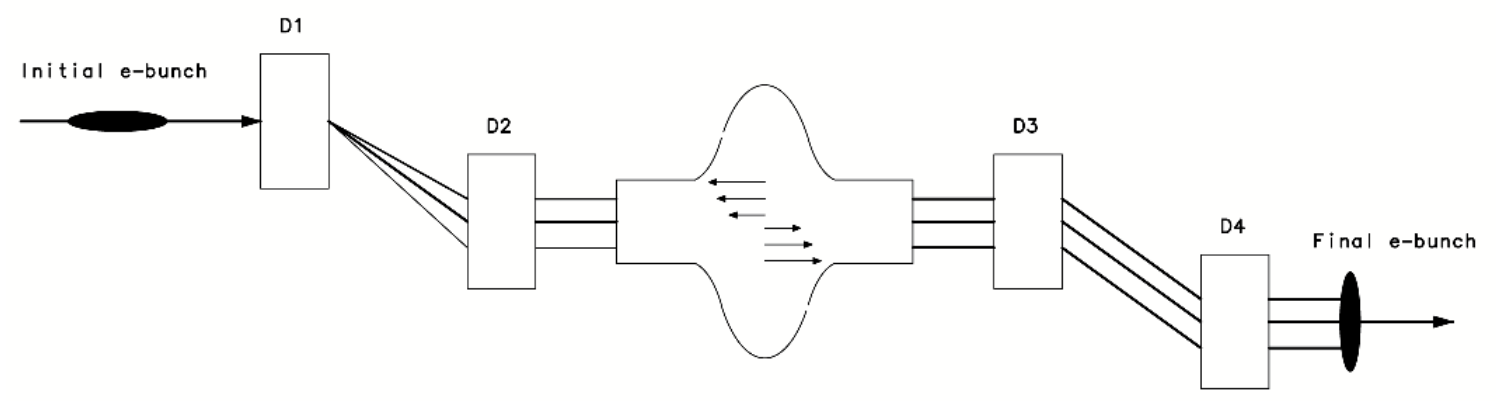

Figure 3.2: Schematic concept of emittance exchange beam line.

$$
M_{E E X}=M_{\text {dogleg }} M_{\text {drift }} M_{\text {cavity }} M_{\text {drift }} M_{\text {dogleg }}
$$

The resulting general emittance exchange matrix reads: 


$$
\left(\begin{array}{cccc}
1+D k & \frac{D}{\alpha}+\frac{D(1+D k)}{\alpha}+l+(1+D k) l+D k\left(\frac{D}{\alpha}+l\right) & k\left(\frac{D}{\alpha}+l\right) & D+D(1+D k)+\alpha D k\left(\frac{D}{\alpha}+l\right) \\
0 & 1+D k & k & \alpha D k \\
\alpha D k & D+D^{2} k+D(1+D k)+\alpha D k l & 1+D k & \alpha D^{2} k+\alpha D(1+D k) \\
k & \frac{D k}{\alpha}+k l & o & 1+D k
\end{array}\right)
$$

Two interesting scenarios come about, first, the trivial $T M_{110}$ Mode Cavity off case where $k=0$, which is just the expected result of two doglegs, each generating dispersion $D$ separated by $2 l$. The more interesting case occurs when $k=\frac{-1}{D} \equiv k_{o}$ the $M_{E E X}$ reads:

$$
M_{E E X}\left(k=k_{o}\right)=\left(\begin{array}{cccc}
0 & 0 & -\frac{1}{\alpha}-\frac{l}{D} & -\alpha l \\
0 & 0 & -\frac{1}{D} & -\alpha \\
-\alpha & -\alpha l & 0 & 0 \\
-\frac{1}{D} & -\frac{1}{\alpha}-\frac{l}{D} & 0 & 0
\end{array}\right)
$$

Notably the on diagonal sub-block elements are zero. Thus $\sigma_{z_{i n}} \cdot \sigma_{z_{i n}^{\prime}}$ phase space area is transported to the $\sigma_{x_{\text {out }}} \cdot \sigma_{x_{\text {out }}^{\prime}}$ area and vice versa for $\sigma_{x_{\text {in }}} \cdot \sigma_{x_{\text {in }}^{\prime}}$ and $\sigma_{z_{\text {out }}} \cdot \sigma_{z_{\text {out }}^{\prime}}$ from which an emittance exchange is apparent: $\epsilon_{x_{\text {in }}} \Rightarrow \epsilon_{z_{\text {out }}}$ and $\epsilon_{z_{\text {in }}} \Rightarrow \epsilon_{x_{\text {out }}}$.

\subsection{Thin vs. Thick Lens $T M_{110}$ Mode Cavity}

Next we must consider the impact of a finite length $T M_{110}$ Mode Cavity on the emittance exchange. First we will derive the equations of motion in a single pillbox resonator from which we will obtain the transfer matrix. Then since the actual $T M_{110}$ Mode Cavity is a 5 -Cell $\pi$-mode structure, we will develop the thick lens cavity from five pillbox resonators, each being $\lambda / 2$ in length, stacked end to end. This closely follows the treatment of Don Edwards [16].

\subsubsection{Equations of Motion in a Pillbox Resonator}

First we define the coordinate system. We use $s$ as the usual longitudinal coordinate and place $s=0$ at the center of the $\lambda / 2$ long resonator. Next we define $X$ as the transverse 
coordinate for the motion, and $X_{r}$ as the transverse position of the reference particle. Thus $x \equiv X-X_{r}$ is the displacement with respect to the reference particle. Motion is only considered in the horizontal and longitudinal directions, therefore $Y$ 's only appearance is to indicate the vertical magnetic field. As was seen above, and more thoroughly described in Chapter 5, the longitudinal electric field has a linear transverse dependence in the region experienced by the particle, thus we define $E^{\prime}=\partial E_{z} / \partial X$ which is then taken to be constant.

$$
\begin{aligned}
& E_{s}(X, t)=E^{\prime} X \cos (\omega t) \\
& B_{Y}(X, t)=\frac{E^{\prime}}{\omega} \sin (\omega t)
\end{aligned}
$$

Applying $\frac{d \vec{p}}{d t}=q(\vec{E}+\vec{v} \times \vec{B})$, we can write:

$$
\begin{gathered}
\frac{d P_{X}}{d t}=-e E^{\prime} \frac{V_{s}}{\omega} \sin (\omega t) \\
\frac{d P_{s}}{d t}=e E^{\prime} X \cos (\omega t)+e E^{\prime} \frac{V_{X}}{\omega} \sin (\omega t)
\end{gathered}
$$

At this point, we can recreate Equation 3.2 from Equation 3.13 in the high frequency limit. First, we make a change of variable, $\theta=\omega t$. In the thin lens, high frequency limit for the $\pi$-mode $(\lambda / 2$ long) cavity, Equation 3.13 becomes:

$$
\frac{d P_{s}}{d \theta}=\frac{e E^{\prime}}{\omega} X \cos (\theta)-\frac{e E^{\prime} V_{s}}{\omega^{2}} \sin (\theta)
$$

Since the second term goes to zero in the limit of high frequency, integration of the remaining first term yields the change in fractional momentum, $\delta \equiv \Delta P_{s} / P_{o}$ with horizontal offset $X$ :

$$
\frac{\Delta P_{s}}{P_{o}}=\frac{2}{\pi} \frac{e E^{\prime}(\lambda / 2)}{p c} X=k X
$$

where $2 / p i$ is the transit time factor for a $/ p i$ mode cavity of length $\lambda / 2$. The cavity strength, $k$, is then defined to be: 


$$
k \equiv \frac{e E^{\prime}}{p c} \frac{\lambda}{\pi}
$$

which is equivalent to the thin lens strength defined above in Equation 3.2, normalized to the central momentum $p c \equiv P_{o}$. We can estimate the change in transverse position through the thick lens cavity. Assume the particle enters the cavity on axis without an initial deflection, i.e. $X=0$ and $d X / d t=0$, such that $V_{X} d \gamma / d t$ is negligible in comparison to $\gamma d V_{X} / d t$, and take $v_{s}=c$, we can write:

$$
\frac{d^{2} X}{d \theta^{2}} \approx-\frac{k \lambda^{2}}{8 \pi^{2}} \sin \theta \rightarrow X=\frac{k \lambda^{2}}{4 \pi^{2}}\left(\frac{1+\sin (\theta)}{2}\right)
$$

thus, at the exit of the cavity, the horizontal displacement can be written as:

$$
X_{\text {exit }}=\frac{k \lambda^{2}}{4 \pi^{2}}
$$

We can estimate the effect in our setup for a single pillbox cavity, by setting the cavity strength to equal the negative reciprocal of the dispersion at the cavity location, $k=-1 / D$, where $D=0.33 \mathrm{~m}$ and $\lambda$ of our $3.9 \mathrm{GHz}$ resonator is $0.077 \mathrm{~m}$. Equation 3.18 yields a horizontal displacement $-0.45 \mathrm{~mm}$. Differences in longitudinal path lengths are significantly smaller.

\subsubsection{Transfer Matrix of a Pillbox Resonator}

$$
\begin{gathered}
E_{s}=E^{\prime} X \cos \left(\omega t-\frac{2 \pi z}{\lambda}\right) \approx E^{\prime} X \cos (\omega t)+E^{\prime} X \frac{2 \pi z}{\lambda} \sin (\omega t) \\
B_{y}=\frac{E^{\prime}}{\omega} \sin \left(\omega t-\frac{2 \pi z}{\lambda}\right) \approx \frac{E^{\prime}}{\omega} \sin (\omega t)-\frac{E^{\prime}}{c} z \cos (\omega t)
\end{gathered}
$$

$E^{\prime}$ defined earlier, change the independent variable to $s=v_{s} t \approx c t$ and use $\omega=\frac{2 \pi c}{\lambda}$, the equations of motion become:

$$
\frac{d P_{X, r}}{d s}=-\frac{e E^{\prime} \lambda}{2 \pi c} \sin \left(\frac{2 \pi}{\lambda} s\right)
$$




$$
\frac{d P_{s, r}}{d s}=e E^{\prime} X_{r} \cos \left(\frac{2 \pi}{\lambda} s\right)+e E^{\prime} V_{X, r} \frac{\lambda}{2 \pi c} \sin \left(\frac{2 \pi}{\lambda} s\right)
$$

Neglecting second order terms, the equations for a neighboring particle are:

$$
\begin{aligned}
& \frac{d P_{X}}{d s}=-\frac{e E^{\prime} \lambda}{2 \pi c} \sin \left(\frac{2 \pi}{\lambda} s\right)+\frac{e E^{\prime}}{c} z \cos \left(\frac{2 \pi}{\lambda} s\right) \\
& \frac{d P_{s}}{d s}=\frac{e E^{\prime}}{c} X \cos \left(\frac{2 \pi}{\lambda} s\right)+\frac{e E^{\prime} \lambda}{2 \pi c^{2}} V_{x} \sin \left(\frac{2 \pi}{\lambda} s\right)
\end{aligned}
$$

We take the difference of Equations 3.21-3.23 and Equations 3.22-3.24, and write:

$$
\begin{gathered}
\frac{d p_{x}}{d s}=\frac{e E^{\prime}}{c} z \cos \left(\frac{2 \pi}{\lambda} s\right) \\
\frac{d p_{s}}{d s}=\frac{e E^{\prime}}{c} x \cos \left(\frac{2 \pi}{\lambda} s\right)+\frac{e E^{\prime} \lambda}{2 \pi c} \frac{d x}{d s} \sin \left(\frac{2 \pi}{\lambda} s\right)
\end{gathered}
$$

The equations of motion are finalized by

$$
\frac{d x}{d s}=\frac{p_{x}}{p_{i n}}
$$

where $p_{i n}$ is the incoming longitudinal momentum,

$$
\frac{d z}{d s}=\frac{e B_{y}}{p_{i n}} X \approx \frac{e E^{\prime} \lambda}{p_{i n} 2 \pi c} x \sin \left(\frac{2 \pi}{\lambda} s\right)
$$

Integrating Equation 3.25:

$$
p_{x}(s)=p_{x}(-\lambda / 4)+\frac{e E^{\prime} \lambda}{2 \pi c} z\left[\sin \left(\frac{2 \pi}{\lambda} s\right)+1\right]
$$

Using our definition of $x^{\prime}$, we write:

$$
x^{\prime} \equiv \frac{p_{x}}{p_{s}}=\frac{\gamma m v_{x}}{\gamma m v_{s}}=\frac{1}{v_{s}} \frac{d x}{d t}=\frac{d x}{d s}
$$

Equation 3.29 can be written as: 


$$
x^{\prime}(s)=x_{i n}^{\prime}+\frac{1}{2} k z \sin \left(\frac{2 \pi}{\lambda} s\right)+\frac{k z}{2}
$$

where $x_{i n}^{\prime}$ is the transverse input angle when entering the cavity. Accordingly, integration of Equation 3.31 yields:

$$
x s=x_{i n}+\left(x_{i n}^{\prime}+\frac{k}{2} z\right)\left(s+\frac{\lambda}{4}\right)-\frac{k \lambda}{4 \pi} z \cos \left(\frac{2 \pi}{\lambda} s\right)
$$

Using the results of Equations 3.31 and 3.32 we can integrate Equation 3.26 to solve for the change in $\Delta p / p_{o}$, where again $p_{o}$ is the central momentum, which is taken to be the momentum of the reference particle.

$$
\begin{aligned}
\frac{\Delta p}{p_{o}} \equiv \frac{p_{s}-p_{o}}{p_{o}} & =\left(\frac{\delta p}{p}\right)_{i n}+x_{i n} \frac{k}{2}\left[1+\sin \left(\frac{2 \pi}{\lambda} s\right)\right] \\
& +x_{i n}^{\prime} \frac{k}{2}\left[\left(s+\frac{\lambda}{4}\right) \sin \left(\frac{2 \pi}{\lambda} s\right)\right] \\
& +z_{i n} \frac{k^{2}}{4}\left[\left(s+\frac{\lambda}{4}\right) \sin \left(\frac{2 \pi}{\lambda} s\right)+\frac{1}{k} \cos \left(\frac{2 \pi}{\lambda} s\right)-\frac{\lambda}{4 \pi} \sin \left(\frac{4 \pi}{\lambda} s\right)\right](3.3
\end{aligned}
$$

We can represent Equations 3.30 through 3.35 as:

$$
\left(\begin{array}{c}
x \\
x^{\prime} \\
z \\
(\delta p / p)
\end{array}\right)_{\text {out }}=\left(\begin{array}{c}
x_{i n}+\frac{\lambda}{2} x_{i n}^{\prime}+\frac{\lambda}{4} k z_{i n} \\
x_{i n}^{\prime}+k z_{i n} \\
z_{i n} \\
(\delta p / p)_{i n}+k x_{i n}+\frac{k \lambda}{4} x_{i n}^{\prime}+\frac{k^{2} \lambda}{8} z_{i n}
\end{array}\right)
$$

from which we can extract the thick lens single pillbox cavity:

$$
M_{\text {cavity,pillbox }}=\left(\begin{array}{cccc}
1 & \lambda / 2 & k \lambda / 4 & 0 \\
0 & 1 & k & 0 \\
0 & 0 & 1 & 0 \\
k & k \lambda / 4 & k^{2} \lambda / 8 & 1
\end{array}\right)
$$

Next we assemble a five cell cavity composed of five $\lambda / 2$ pillboxes, each at a strength of $k / 5$, stacked end-to-end: 


$$
M_{\text {cavity,5pillbox }}=\left(\begin{array}{cccc}
1 & 5 \lambda / 2 & 5 k \lambda / 4 & 0 \\
0 & 1 & k & 0 \\
0 & 0 & 1 & 0 \\
k & 5 k \lambda / 4 & 17 k^{2} \lambda / 40 & 1
\end{array}\right)
$$

This is the matrix that will be used in the Trace 3D-MatLab Calculations described in Section 3.4. But first, we build the emittance exchange beamline of the dogleg matrix $M_{d}$, followed by a drift matrix $M_{l 1}$ of length $l_{1}$, the the 5 -Cell pillbox cavity matrix, $M_{c a v}^{5}$, another drift matrix $M_{l 2}$ of length $l_{2}$, and finally the second dogleg matrix $M_{d}$. As was done in the thin lens case, we set the strength of the cavity to be the negative reciprocal of the dispersion at the cavity, $k=-1 / D$, and write:

$$
M_{E E X} \equiv M_{d} M_{l_{2}} M_{c a v}^{5} M_{l_{1}} M_{d}=\left(\begin{array}{cccc}
0 & \frac{17 \lambda}{40} & -\frac{1}{\alpha}-\frac{33 \lambda}{40 D}-\frac{l_{2}}{D} & -\frac{33 \alpha \lambda}{40}-\alpha l_{2} \\
0 & 0 & -\frac{1}{D} & -\alpha \\
-\alpha & -\frac{33 \alpha \lambda}{40}-\alpha l_{1} & \frac{17 \alpha \lambda}{40 D} & \frac{17 \alpha^{2} \lambda}{40} \\
-\frac{1}{D} & -\frac{1}{\alpha}-\frac{33 \lambda}{40 D}-\frac{l_{1}}{D} & \frac{17 \lambda}{40 D^{2}} & \frac{17 \alpha \lambda}{40 D}
\end{array}\right)
$$

It is interesting to numerically evaluate this emittance exchange matrix for comparison later, as shown in Equation 3.40. The drifts $l_{1}$ and $l_{2}$ are taken as the actual experiment values of $640 \mathrm{~mm}$. With the exception of the $R_{43}$ element we arrive at a matrix very close to the desired $M_{E E X}$ form. Additionally, this is very close to thin lens cavity calculation, but the finite length of the cavity causes the $R_{43}$ to become populated.

$$
M_{d} M_{l_{2}} M_{c a v}^{5} M_{l_{1}} M_{d}=\left(\begin{array}{cccc}
0.000 & 0.033 & 4.68 & -0.276 \\
0.000 & 0.000 & 3.030 & -0.393 \\
-0.393 & -0.276 & 0.039 & 0.005 \\
-3.030 & -4.679 & 0.301 & 0.039
\end{array}\right)
$$




\subsection{Approximation to a Thick Lens}

It is interesting to compare the above 5-cell pillbox result, 3.38, with a thick lens approximated by a structure composed of five thin lens cavities, Equation 3.5, each of strength $k / 5$ separated by a drift $\lambda / 2$, and with end-drifts $\lambda / 4$ on each side 3.41 . Other than the $M_{\text {cav }}$ 's $(4,3)$ element, they are identical, and the $M_{\text {cav:4,3 }}$ only varies by $6.3 \%$.

$$
M_{\text {cavity,5thinlens }}=\left(\begin{array}{cccc}
1 & 5 \lambda / 2 & 5 k \lambda / 4 & 0 \\
0 & 1 & k & 0 \\
0 & 0 & 1 & 0 \\
k & 5 k \lambda / 4 & 2 k^{2} \lambda / 5 & 1
\end{array}\right)
$$

\subsection{1 $T M_{110}$ Mode Cavity Matrix From Field Maps}

Finally, a cavity transport matrix was obtained by simulation of our $T M_{110}$ Mode Cavity geometry. This was the work of A0 Photoinjector summer student E. Branlard.[18] A complete set of $E$ and $B$ field maps were generated by the 4-D electromagnetic solver code HFSS, [54]. The field maps were imported into Astra [23] and scaled to match the EEX operating gradient for our experiment. Electrons of differing input conditions were flown in the $T M_{110}$ Mode Cavity fields and the changes in the electron's exit trajectories were recorded. Noting the difference between the input and output beam parameters the transport matrix was deduced. This procedure is equivalent to the method of difference orbits used in measuring the transport matrix of the actual beamline, and is thoroughly discussed in Chapter 6. Branlard's results are displayed in Equation 3.42:

$$
M_{\text {cavity,HFSS }}=\left(\begin{array}{cccc}
1.003 & 0.192 & 0.286 & 0.001 \\
0.020 & 1.004 & 2.991 & 0.000 \\
0.001 & 0.001 & 0.998 & 0.000 \\
3.000 & 0.288 & 0.275 & 0.999
\end{array}\right)
$$

This compares with the numerical evaluations of both Equations 3.38 and 3.41: 
First Equation 3.38 evaluated:

$$
M_{\text {cav,5pillbox }}=\left(\begin{array}{cccc}
1.000 & 0.192 & 0.291 & 0.000 \\
0.000 & 1.000 & 3.030 & 0.000 \\
0.000 & 0.001 & 1.00 & 0.000 \\
3.030 & 0.291 & 0.300 & 1.000
\end{array}\right)
$$

and Equation 3.41 when evaluated:

$$
M_{\text {cav,5thinlens }}=\left(\begin{array}{cccc}
1.000 & 0.192 & 0.291 & 0.000 \\
0.000 & 1.000 & 3.030 & 0.000 \\
0.000 & 0.001 & 1.000 & 0.000 \\
3.030 & 0.291 & 0.283 & 1.000
\end{array}\right)
$$

There is remarkable agreement between the thick lens built up from five thin lenses, the 5-Cell pillbox model, and the calculated fields of the actual $T M_{110}$ Mode Cavity geometry.

\subsection{Trace 3D Simulations}

Trace 3D (T3D) is a Matrix Calculation code developed by Los Alamos to aid in the design of a beam transport system. A user defined beamline can be constructed from T3D's library of beam elements, e.g. dipole magnets, quadrupole magnets, and standard accelerating cavities. The reader is referred to Figure 4.11 for placement of beamline elements. A standard T3D input file is listed in Appendix D. Unfortunately, our $T M_{110}$ Mode Cavity is not included in this library and thus had to be externally implemented as described below. The T3D elements are treated as thick lenses; the user defined magnetic elements provide a soft edge approximation to account for fringe fields.

Full 3-D input beam parameters, such as the emittance and Courant-Synder Parameters and beam charge density, can be specified at the start of the beamline and propagated through the beamline model. The evolution of the beam can be plotted at the exit of each element. T3D includes a linear space charge algorithm to approximate beam defocusing and emittance growth due to internal space charge forces. A T3D model of the A0 Photoinjector 
beamline was generated and used by the author as a guiding tool during the measurement of the EEX matrix as well as the observation of the emittance exchange. Others [21] have completed thorough EEX simulations which have also provided guidance for this experiment. We will conclude this conceptual discussion of emittance exchange with the T3D simulations.

\subsubsection{Trace 3D Matrix Model}

A description of the A0 EEX hardware has been developed using a hybrid Trace-3D MatLab model.[19],[20] The optics model matches the physical hardware from which the matrix is measured. As fully described in Chapter 6, we define the EEX matrix to include a drift of 2.2 meters prior to the first dipole and 0.2 meters following the fourth dipole - that is, all focusing quadrupole elements in the beamline are off for the matrix measurement.

This T3D model includes a bending radius of $900 \mathrm{~mm}$ for each of the four $22.5^{\circ}$ bending dipoles and includes the soft edge approximation of a linear field drop to $50 \%$ at a distance of $1 / 2$ the air gap height away from the physical pole tip edge. During nominal operation the dipoles have a measured peak vertical field of $600 \pm 10$ Gauss in the central region of the poletip. The magnetic rigidity, $\mathrm{B} \rho$, for a $14.3 \mathrm{MeV} / \mathrm{c}$ beam is $0.047 \mathrm{~T} \cdot \mathrm{m}$, thus in an ideal magnet, void of fringe fields, the bending radius is $783 \mathrm{~mm}$, imparting an $\int B d l=$ $0.018 \mathrm{~T} \cdot \mathrm{m}$. However as discussed in Chapter 4 the greatly extending fringe fields measured between the first and second dipoles indicate a distributed bending region that greatly exceeds the pole tip boundaries. Since T3D does not have a capacity for complicated field geometries, our T3D model incorporates an ideal magnet with a bending radius of $\rho=900$ $\mathrm{mm}$, bending through $22.5^{\circ}$, which has the reduced average vertical magnetic field of 522 gauss to accomplish an $\int B d l=0.018 \mathrm{~T} \cdot \mathrm{m}$.

All of the static beamline elements were modeled in Trace 3D, using a drift of the $T M_{110}$ Mode Cavity length in place of the cavity - it is replaced by a cavity matrix in the MatLab code. A pertinent sequence of matrices describing the entire beamline was generated by T3D and imported into MatLab code. The MatLab model simply replaces the T3D drift length with the previously described thick lens cavity approximated by five pillbox resonators stacked end-to-end. The complete beamline matrix calculations, including the 
$T M_{110}$ Mode Cavity of strength $k$, are performed in the MatLab code. Equation 3.45 is the numerical $M_{E E X}$ calculated by this T3D-MatLab with the cavity on at $100 \% k \equiv k_{o}$. The $4 \times 4$ elements of the $M_{E E X}$ were then plotted against a $T M_{110}$ Mode Cavity of varied strength in Figure 3.3. In Figure 3.3, the cavity off condition, $k=0$ is denoted as $0 \%$, and the ideal emittance exchange $k=k_{o}$ denoted as $100 \%$. Most notably all of the $A$ and $D$ sub-block elements, except $R_{43}$, tend to zero.

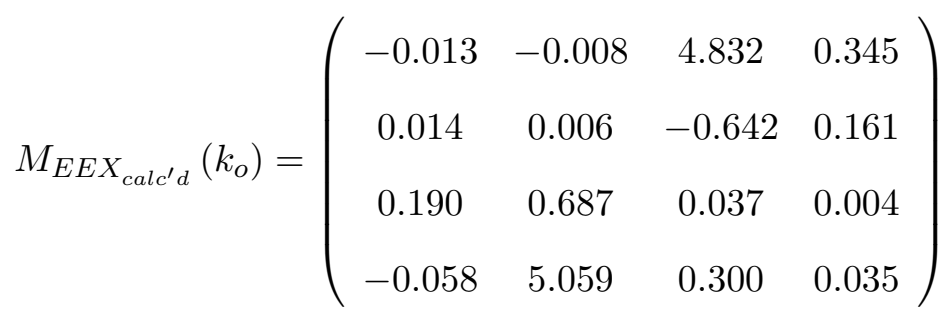

A cautionary note, because of the thick lens bending dipoles and their edge focusing terms, this matrix is not directly comparable to the matrices calculated in Equation 3.40. However, it is interesting to note that the $R_{43}, \partial \delta_{\text {out }} / \partial z_{\text {in }}$, term is the same.

\subsubsection{Trace 3D Simulation for Initial Beam Conditions}

In preparation for the direct emittance exchange measurements, discussed in Chapter 7, a cathode-to-dump ASTRA-ELEGANT simulation was performed by Fliller [21] for a bunch charge of $1 \mathrm{nC}$. Ultimately the EEX experiment was performed at $0.25 \mathrm{nC}$ hence the input optics were different, but the $1 \mathrm{nC}$ simulations provided a reasonable starting point.

During the $250 \mathrm{pC}$ experimental runs, this author used T3D with the space charge calculations turned on as guidance for input quadrupole settings used for the exchange.

In the following simulation, the beamline starts at $\mathrm{X} 3$ and terminates at the exit of the emittance exchange beamline, defined to be BPM28. Again, the cavity is represented by a drift space, and thus is considered off. The choice T3D input beam parameters were based on actual input beam measurement. During operation the electron bunch charge was selected to be $250 \mathrm{pC}$, the $\mathrm{RF}$ Gun solenoids were adjusted to provide a minimum spot size on X3 viewing screen. Transverse spot size and slit emittance measurements at $\mathrm{X} 3$ yielded the Courant-Synder $\beta_{x, y}$ and $\alpha_{x, y}$. Finally, the longitudinal beam parameters were obtained from the off-crest phase value of the 9-Cell which gave maximum bunch 

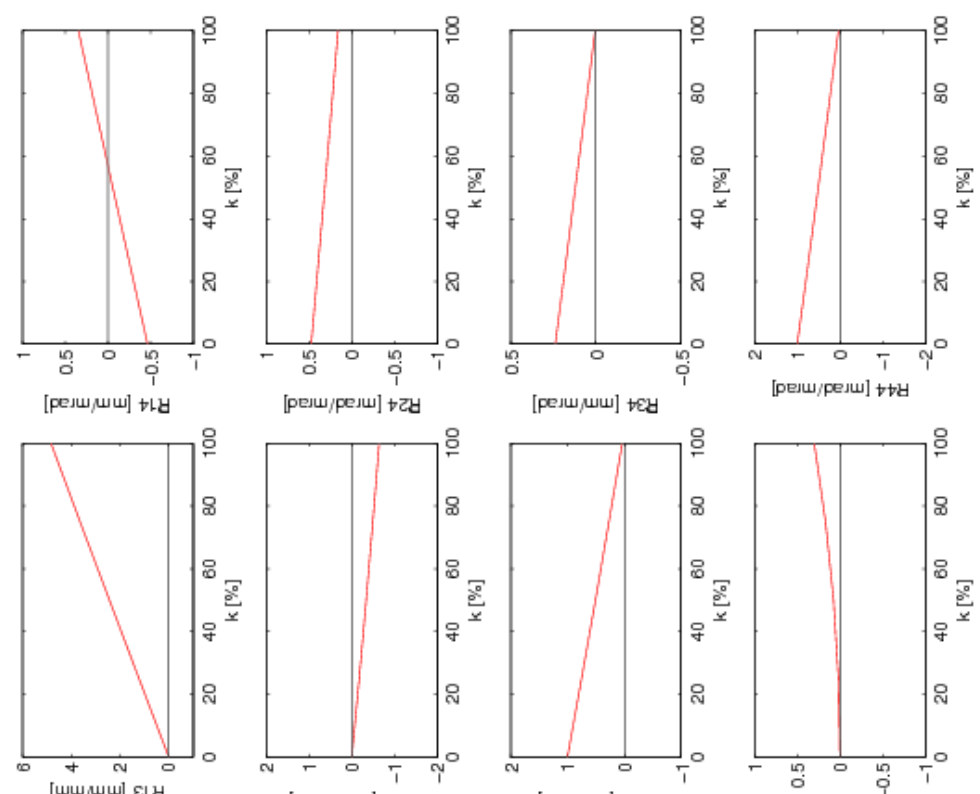

[релш/релш] $\downarrow$ ¿乙4

[релш/шس] tге
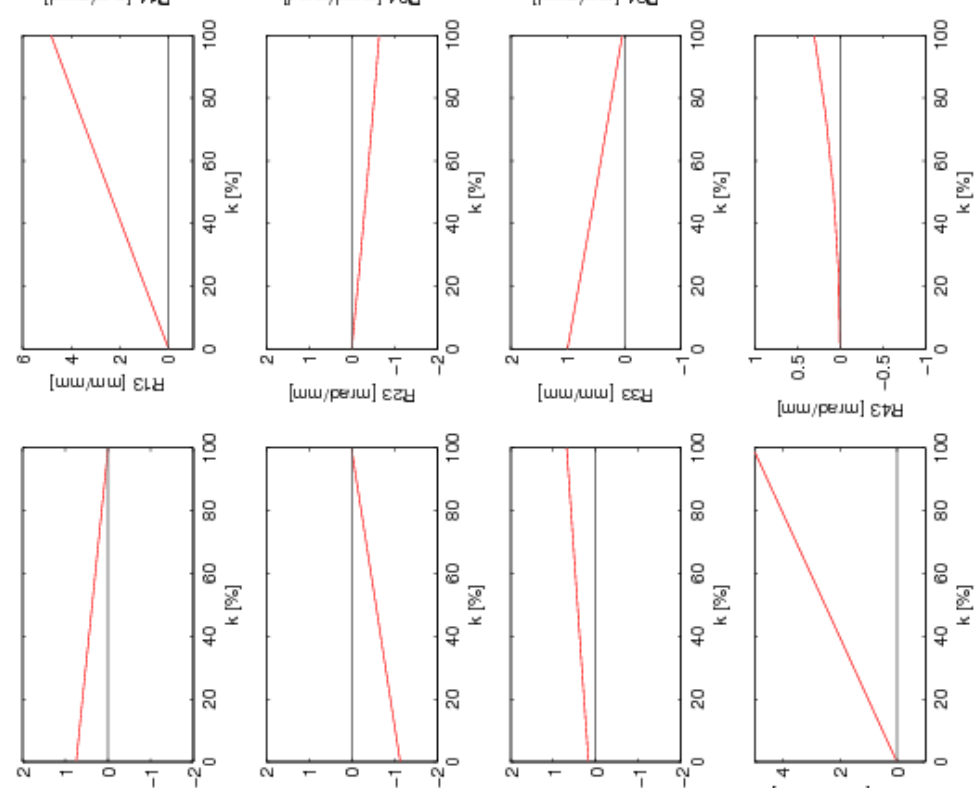

[релш/шس] 난
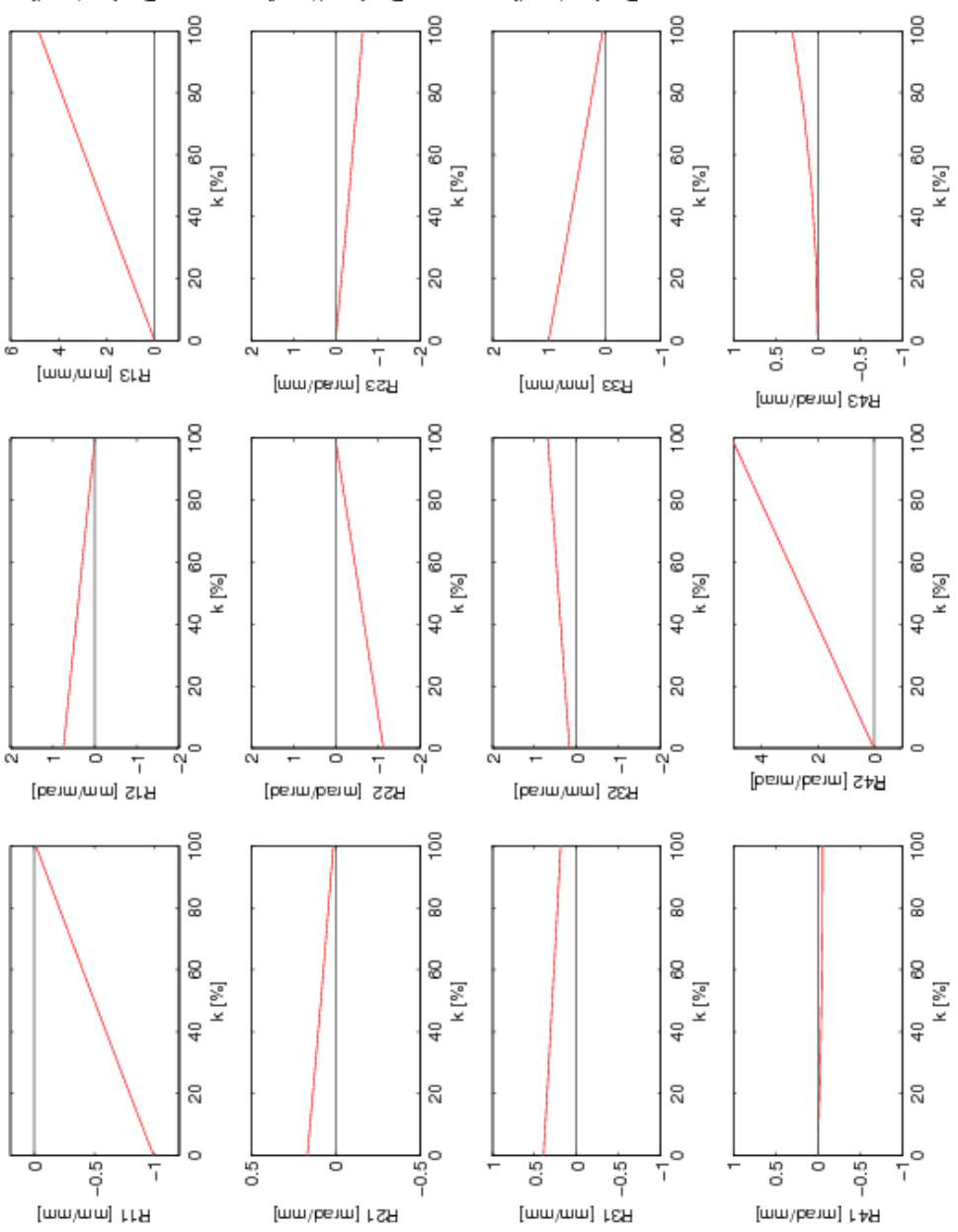

Figure 3.3: Our standard Trace 3D / MatLab EEX beamline transport matrix, starting from HTBX03 and ending at BPM29 (Refer to Figure 4.11) for cavity strengths $k=0$, off, to $k_{o}=100 \% k$, the ideal emittance exchange strength.(Note image is rotated $90^{\circ}$ for viewing) 


\begin{tabular}{|c|c|c|}
\hline X3 Input Parameter & Value & T3D unit \\
\hline$\alpha_{x}$ & 0 & \\
\hline$\beta_{x}$ & 0.17 & $\mathrm{~mm} / \mathrm{mrad}$ \\
\hline$\alpha_{y}$ & 0 & \\
\hline$\beta_{y}$ & 0.17 & $\mathrm{~mm} / \mathrm{mrad}$ \\
\hline$\alpha_{\phi}$ & -3.0 & \\
\hline$\beta_{p h i}$ & 0.0842 & $\mathrm{deg} / \mathrm{keV}$ \\
\hline Momentum & 14.3 & $\mathrm{MeV} / \mathrm{c}$ \\
\hline$\epsilon_{x}$ & 3.5 & $\mathrm{~mm} \cdot \mathrm{mrad}$ \\
\hline$\epsilon_{y}$ & 3.5 & $\mathrm{~mm} \cdot \mathrm{mrad}$ \\
\hline$\epsilon_{z}$ & 482 & $\mathrm{deg} \cdot \mathrm{keV}$ \\
\hline RF Frequency & 1300 & $\mathrm{MHz}$ \\
\hline
\end{tabular}

Table 3.1: X3 T3D beam input parameters.

compression at X24. Momentum and momentum spread measurements were obtained from the XS3 Spectrometer. The input conditions in T3D units are summarized in Table 3.1.

It is useful to first propagate the input beam through the beamline with all of the focusing quadrupole magnets off to demonstrate the inherent focusing properties of the bending dipole's edge angles.

Next, we supplement the edge focusing with the use of the first three quadrupoles in the beamline after X3, namely Q1AX03, Q1AX05, and Q2AX06. For the simulation run displayed in Figure 3.4, the input beam momentum spread is fictitiously set to zero to obtain the $\beta$-function's contribution to the beam envelope, i.e.:

$$
\sigma_{x}=\sqrt{\epsilon_{x} \beta_{x}+D_{x}^{2}\left\langle\left(\frac{\Delta p}{p_{o}}\right)^{2}\right\rangle}=\sqrt{\epsilon_{x} \beta_{x}+0}
$$

With the choice of Q1AX03=- 0.23 amperes, Q1AX05 $=-0.76$ amperes, and Q2AX06 $=0.35$ amperes, a beam waist in the horizontal plane developed in the center of the $T M_{110}$ Mode Cavity . Next, the momentum spread was restored and the contribution from the momentum spread was noted.

The three quadrupoles used to adjust the transverse input condtions: Q1AX03, Q1AX05, and Q2AX06. The first quadrupole, Q1AX03, was held fixed based on Fliller's simulation. Only second two were scanned to limit the parameter space. With the beam's momentum spread zeroed in the simulation to isolate the $\beta$-function, last two quadrupoles were scanned to minimize the horizontal spot at the center of the $T M_{110}$ Mode Cavity . Having 


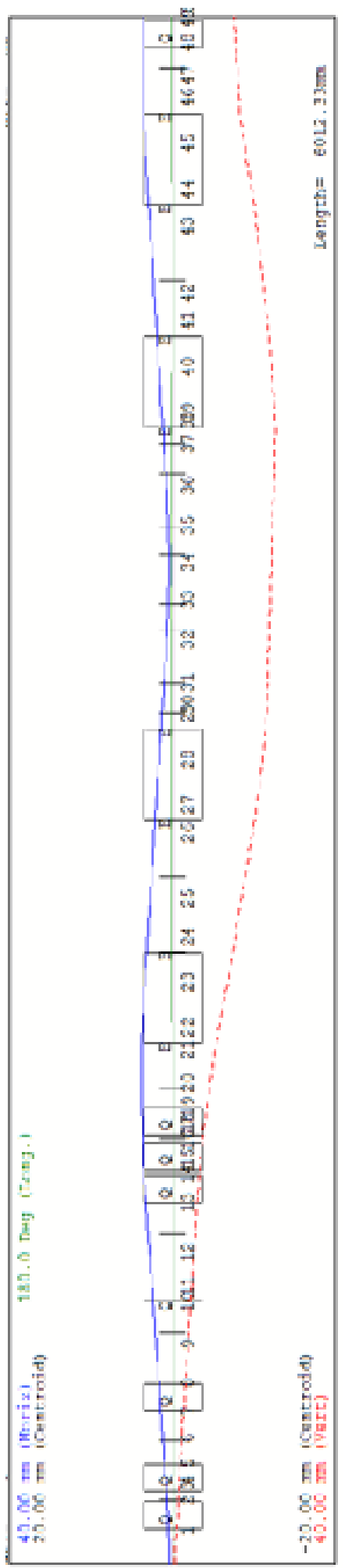

Figure 3.4: T3D Simulation plotting the horizontal beam envelope along the EEX beamline of a mono-energetic beam. The blue trace is the horizontal beam envelope, while the red trace is the vertical beam envelope. Note image is rotated 90 degrees for ease of viewing. 
the particles' transverse offset at the $T M_{110}$ Mode Cavity dominated by momentum offset, an energy- $\beta$-function correlation is reduced. 


\section{Chapter 4}

\section{Experimental Apparatus \& Diagnostics}

\subsection{A0 Photoinjector Front End}

The A0 Photoinjector, partially named for the Fermilab building in which it resides, is an advanced accelerator research and development facility with a concentration on beam manipulations. The Photoinjector began operation in 1998 and has been the source of many accelerator physics PhD's. In addition to numerous beam physics theses, the A0 Photoinjector has brought the technology of superconducting radio frequency to Fermilab, which as a result, is now positioned to significantly contribute to the design and construction of a superconducting linac.

The principle components that define the A0 Photoinjector are a frequency quadrupled UV laser that illuminates a photocathode located in a 1.5 cell $1.3 \mathrm{GHz}$ normal conducting RF photoelectron Gun [24]. The RF gun is followed by a 9-cell $1.3 \mathrm{GHz}$ superconducting TESLA style accelerating cavity [31]. The remainder of the facility is a versatile experimental area whose beamlines can be configured to suit the needs of a particular experiment. An outline of a few past experiments includes plasma wakefield acceleration [32], channeling radiation [33], round-to-flat beam transformation [6], low emittance studies [37].

Again, the reader is referred to Figure 4.11, the schematic layout of the Photoinjector. It displays the relative locations of beamline elements, and will greatly aid the reader during the discussion in this chapter. Tables in Appendix A provide the longitudinal positions for all elements in both EEX and straight ahead beamlines. Table 4.1 summarizes the Photoinjector's major operating parameters. 


\begin{tabular}{|c|c|c|}
\hline Parameter & Value & unit \\
\hline Photocathode & $\mathrm{Cs}_{2} T e$ & \\
\hline Photocathode Quantum Efficiency (typical) & 0.75 & $\%$ \\
\hline Laser (UV) $\lambda$ & 263 & $\mathrm{~nm}$ \\
\hline Laser Fire Phase (wrt gun crest) & 45 & $\mathrm{deg}$. \\
\hline Laser Pulse duration & 2.2 & $\mathrm{ps}(\mathrm{rms})$ \\
\hline Laser on cathode spot size & $3.4(\mathrm{typ})$ & $\mathrm{mm}$ \\
\hline Charge per pulse (tunable) & $0.1-4$ & $\mathrm{nC}$ \\
\hline Gun on Cathode Gradient & 35 & $\mathrm{MV} / \mathrm{m}$ \\
\hline 9-Cell max. accel. gradient & 13 & $\mathrm{MV} / \mathrm{m}$ \\
\hline Bunch spacing & 1 & $\mu s$ \\
\hline RF pulse rep. rate & 1 & $\mathrm{~Hz}$ \\
\hline Transverse norm. emittance $(0.25 \mathrm{nC})$ & 4 & $\mathrm{~mm} \cdot \mathrm{mrad}$ \\
\hline Longitudinal norm. emittance $(0.25 \mathrm{nC})$ & 22 & $\mathrm{~mm} \cdot \mathrm{mrad}$ \\
\hline
\end{tabular}

Table 4.1: A0 9-Cell and Beam Parameters

\subsubsection{The Copper RF Gun}

The second half of the A0 Photoinjector namesake is the RF Photoelectron Gun which consists of a photocathode located inside an RF cavity. The RF structure is a copper 1.5-cell $1.3 \mathrm{GHz}$ resonator operating in the $T M_{010} \pi$-mode. RF power is inductively coupled into the full cell via a side coupled waveguide matching network. The accelerating longitudinal electric field terminates on the back wall of the half cell where the photocathode resides. The on-cathode gradient that develops as function of power has been, calculated by Y.E. Sun [6] and others, shown to be:

$$
E_{\text {cath }}[M V / m]=23.7 \sqrt{P_{\text {incident }}[M W]}
$$

Typical operating conditions are 2.2 MW resulting in a gradient of $35 \mathrm{MV} / \mathrm{m}$. The photocathode is a $\mathrm{Cs}_{2} \mathrm{Te}$ coated Molybdenum plug. An ultraviolet laser illuminates the photocathode generating short, intense electron emission of current densities on order of $\mathrm{kA} / \mathrm{cm}^{2}$, two orders of magnitude greater than achievable with thermionic emitters [39]. The electrons are then rapidly accelerated by the strong longitudinal field, at exit of the gun, the electron momentum is about $3.5 \mathrm{MeV} / \mathrm{c}$.

In addition to providing high charge electron beams, a phase space manipulation early at the RF Gun can greatly compensate for space charge emittance growth, permitting the 


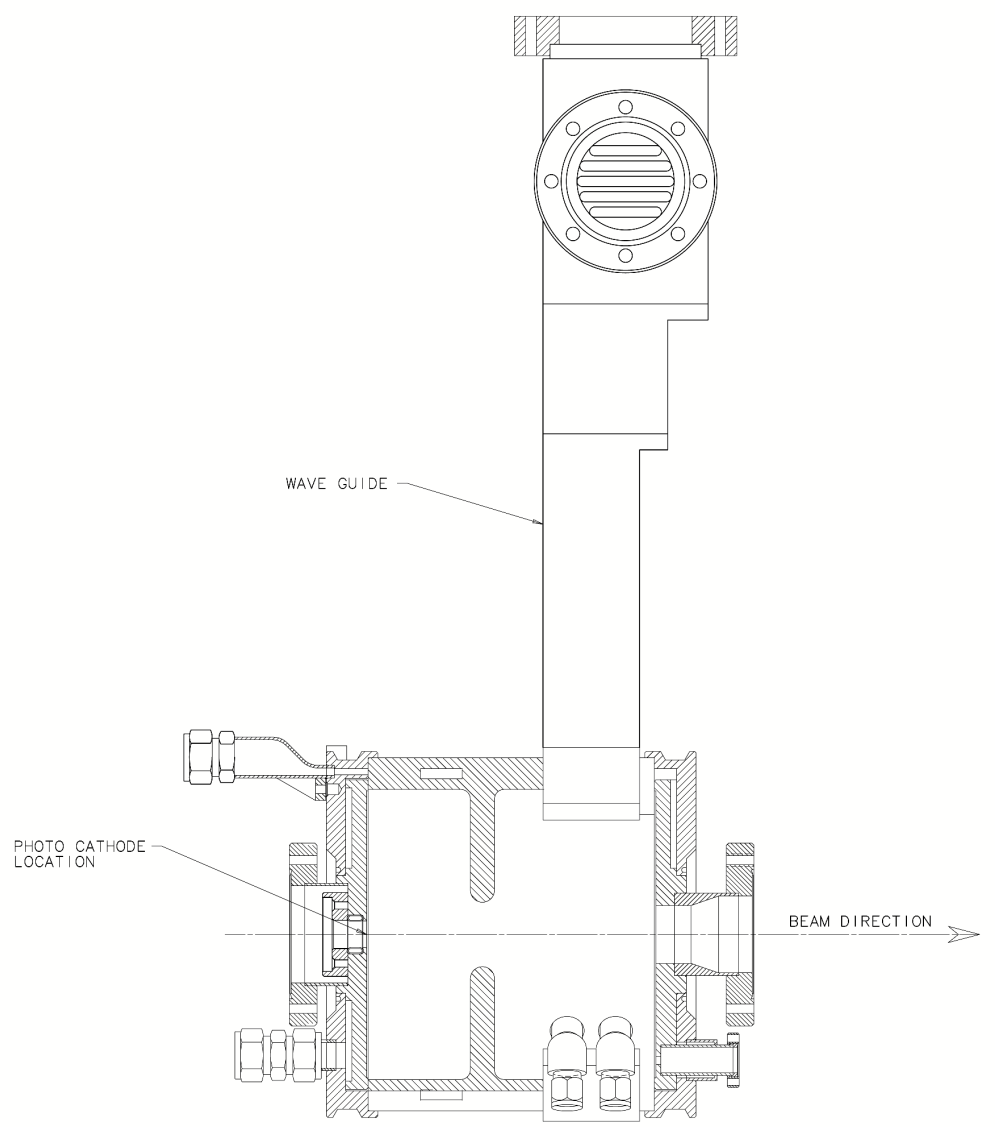

Figure 4.1: Cross Sectional View of RF Gun 

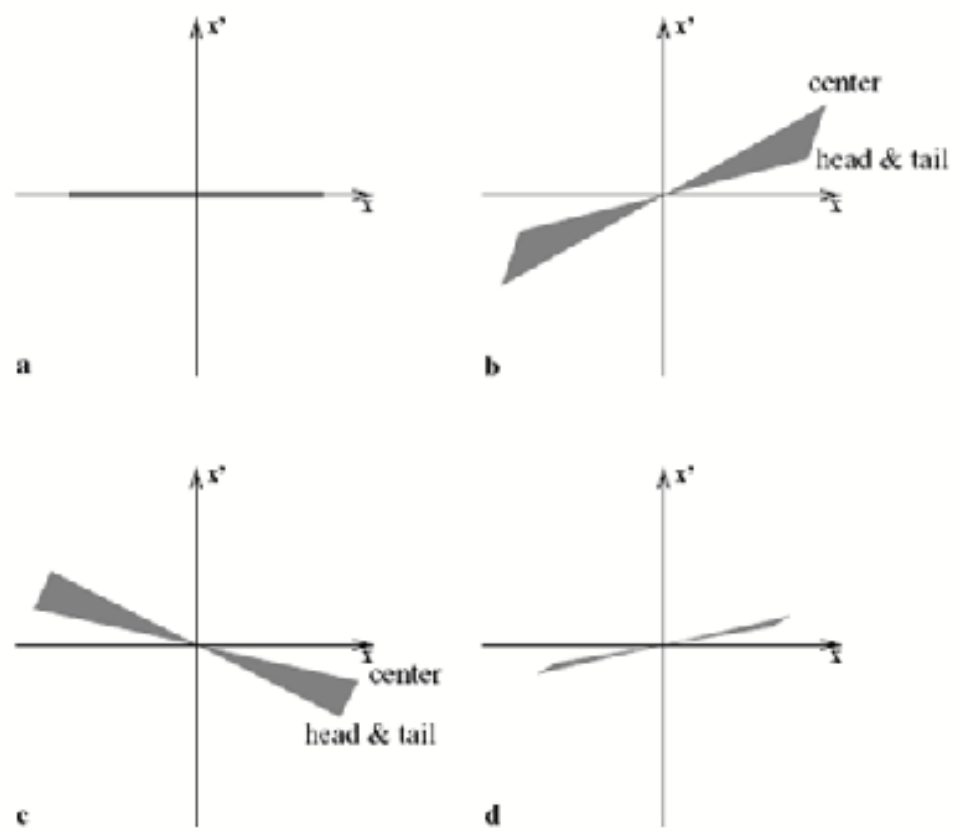

Figure 4.2: Viewing Emittance Compensation in $x-x^{\prime}$ phase space. [38]

generation of extremely low transverse emittance beams. [26]

Several factors contribute to the growth of transverse emittance in an RF gun.[38] The first two are the thermal emittance of the electrons as they are born from the cathode and RF focusing fields of the RF gun. These can be controlled by the laser wavelength and laser spot size on the cathode. However, the more dominant deleterious effect is from internal space charge fields of the high-charge bunches created. The emittance growth of the space charge fields can be compensated by the focusing action of a solenoid field. Thus, the RF Gun is nested in three externally placed solenoids. The space charge forces introduce a radial phase space correlation with $z$ position within the bunch. The central portion of an electron bunch experiences greater radial forces than does the head or tail, thus a central disk of the bunch will have a greater $p_{r}$. A judiciously placed solenoid field can introduce a radial focusing kick that reverses the radial space charge defocusing. After a short drift the transverse projected emittances drops to a minimum, after which the space charge forces would again cause the emittance to grow. At this juncture it is imperative to introduce a strong accelerating gradient to capture and maintain the beam at its lowest projected emittance, hence the naming of the superconducting 9-Cell as Capture Cavity I. 
The emittance compensation process can be viewed in the $x-x^{\prime}$, or equivalently the $y-y^{\prime}$, phase space planes, Figure 4.2. Consider the phase space plot as though one was viewing a ribbon coming edge on; all the viewer can see is the transverse projection of the ribbon - thus at the extremes one sees either a sliver if the ribbon is flat, or a circle when it is completely twisted. As the electron bunch departs the photocathode the phase space plot is narrow (Figure 4.2a), but as the defocusing space charge forces build up and take effect the phase space plot rotates. Although the instantaneous phase space is a thin ellipse, the viewer sees the circle being filled in by the rotation (Figure $4.2 \mathrm{~b}$ ), hence an apparent 'emittance growth.' However, the solenoidal field reverses this twist (Figure 4.2c) and for a moment the projection returns to that of a flat ribbon (Figure $4.2 \mathrm{~d}$ ). The beam is then quickly accelerated to energies where the space charge forces are reduced.

\subsubsection{The 9-Cell Superconducting Capture Cavity}

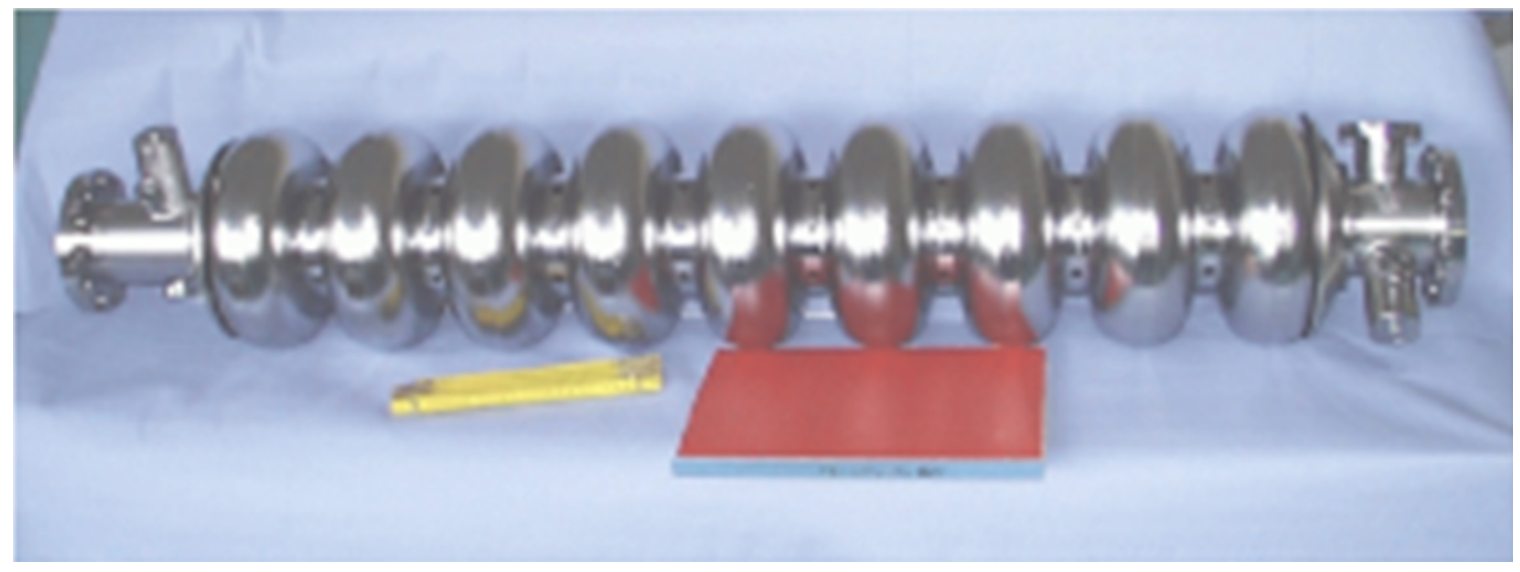

Figure 4.3: TESLA 9-Cell Niobium Cavity

The RF Gun is followed by an early model 9-cell TESLA style superconducting RF cavity [31]. This cavity holds several names, such as Capture Cavity I, the booster cavity, or simply the 9-Cell. The 9-Cell operates in the standing wave $T M_{010}$ 'accelerating' mode. The bare 9-Cell cavity came to Fermilab as part of the TESLA TTF collaboration. It was installed into a cryovessel and was brought into operation during 1998. The maximum achievable accelerating gradient is $13 \mathrm{MV} / \mathrm{m}$. The 9-Cell length is $1028 \mathrm{~mm}$, therefore the maximum final electron beam momentum is $16 \mathrm{MeV} / \mathrm{c}$. The advantage of a superconducting 
cavity becomes apparent in that the 9-cell $\mathrm{RF}$ power requirement is a meager $200 \mathrm{~kW}$, in contrast to the $3 \mathrm{MW}$ consumed by the normal conducting RF gun. As Equation 4.2 demonstrates,

$$
\frac{P}{L}=\frac{E_{a c c}^{2}}{\frac{R}{Q} Q_{o}},
$$

the RF power per unit length is inversely proportional to the cavity's quality factor, $Q_{o}$, for a given accelerating gradient $E_{a c c}$ and cavity shunt impedance, $\frac{R}{Q}$ - a geometrical factor. As will be discussed in Chapter 5 , the $Q_{o}$ is proportional to the cavity wall's surface resistance $R_{s}(f)$. In the case of superconductivity the surface resistance, $R_{s}=R_{B C S}+R_{\text {residual }}$ precipitously drops with temperatures below $T_{c}$ :

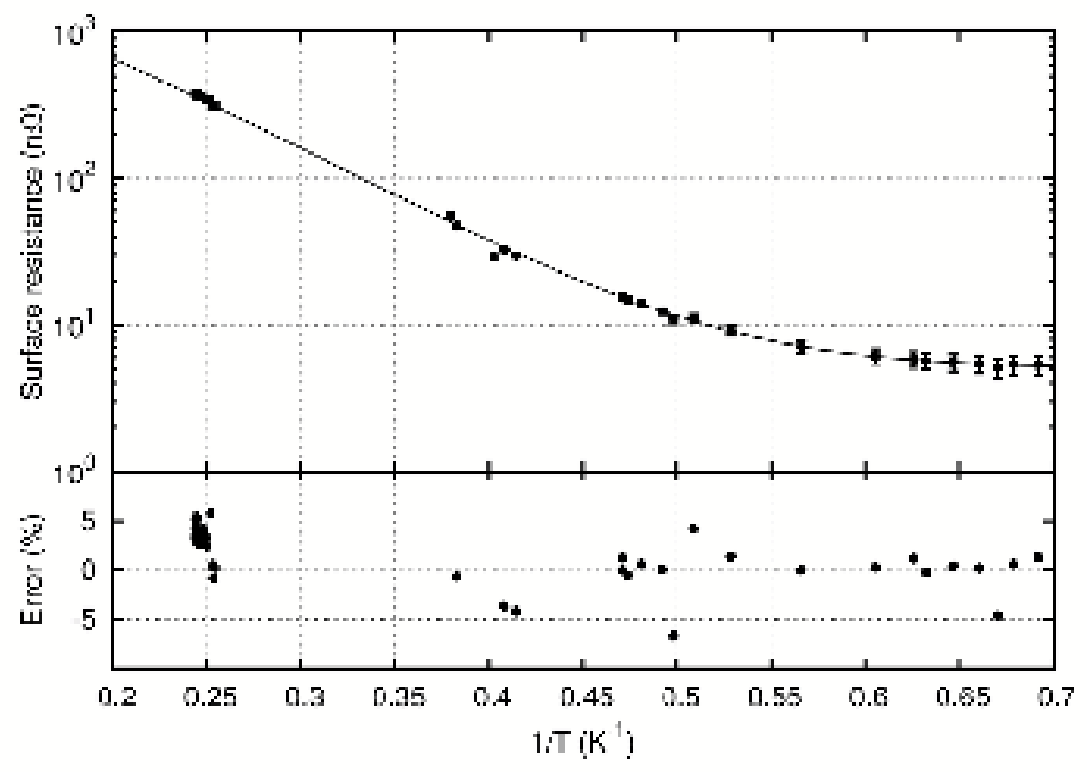

Figure 4.4: TESLA 9-Cell $R_{s}$ as a function of $T_{c} / T$, Niobium $T_{c}$ is $9.2 \mathrm{~K}$. [27]

$$
R_{B C S} \propto \frac{f^{2}}{T} \exp \left(-1.76 T_{c} / T\right)
$$

Where $f$ is the operating frequency. The cavity is manufactured out of pure Niobium, which transitions to superconductivity at 9.2 K. Further, as demonstrated by Equation 4.3, $R_{s}$ drops from $800 \mathrm{n} \Omega$ at $4.2 \mathrm{~K}$ to $15 \mathrm{n} \Omega$ at $2 \mathrm{~K}$, see Figure 4.4. The reduction in $R_{s}$ of Equation 4.3 improves the resonator's $Q_{o}$ to values of $10^{10}$ or better. The $Q_{\text {loaded }}$ is 
determined by the cavity input coupler. Its coupling is chosen on the basis of cavity fill time, RF regulation, anticipated beam current, etc. For more information on the 9-Cell input coupler, see Appendix B.

\subsection{Integrated RF Systems}

The line between High Level RF (HLRF) and Low Level RF (LLRF) responsibilities is approximately between the output of the LLRF controller, at a power of $\approx 10 \mathrm{dBm}$ $(30 \mathrm{~mW})$, and the input to the power amplifier chain. However, at Fermilab, there frequently is a collegial overlap and exchange of talent between the two sub-disciplines. A significant infrastructure of both HLRF and LLRF is needed to safely and reliably operate the RF Gun, the Superconducting 9-Cell, and the 3.9 GHz Klystron. The following subsections are an overview of the components supporting the $T M_{110}$ Mode Cavity operation, germane to the EEX beamline; However, they are also representative of the RF Gun and 9-Cell HLRF and LLRF systems.

\subsection{1 $T M_{110}$ Mode Cavity High Level RF}

The six principle components of the HLRF system include the charging supply and capacitor bank, the series-tube modulator, the Klystron, the transmission line, the input coupler, and an extensive machine-protection system - these are schematically diagrammed in Figure 4.5. Because of this unique application, most of the systems are designed and built in-house, however, standard components, such as waveguide and directional couplers are purchased radar surplus items. The expensive vacuum tubes, including the Klystron and the 4CW100,100D have to be purchased new or rebuilt.

During operation, the Klystron gun is pulsed with $38 \mathrm{kV}$ by a series tube modulator. The pulse, up to $1.4 \mathrm{~ms}$ long, is generated by a EIMAC 4CW100,000D tetrode wired in series between a $40 \mathrm{kV}, 6 \mu \mathrm{F}$ capacitor bank and the Klystron gun. The Klystron $38 \mathrm{kV}$, 6 Ampere pulse is formed by control circuitry biasing the control grid of 4CW100,000D sufficiently to send the tube into conduction. Additionally, during the modulator pulse, a feedback circuit adjusts the bias on the 4CW100,000D control grid, which in turn adjusts 
the tetrode's series impedance. Reduction in the tube's series impedance compensates for the voltage droop of the capacitor bank. The initial $2 \mathrm{kV}$ difference of the capacitor bank provides the needed overhead for regulation during the pulse.

The Klystron's output RF power is transported into the A0 Photoinjector's bunker by WR284 waveguide. The Klystron is reverse power protected by a ferrite circulator inserted into the waveguide. Several directional couplers for measuring forward and reflected power are installed in the waveguide run. There is a directional coupler immediately before the cavity's input coupler which is used for measuring the $P_{f w d}$ and $P_{r e f}$ during the $T M_{110}$ cavity operation. As a personnel safety feature, the WR284 size waveguide run between the Klystron and the cavity is hermetically sealed, slightly pressurized with dry nitrogen, and interlocked to inhibit the modulator during loss of pressure. Thus RF can not be generated if the waveguide is not sealed. Other HLRF equipment protection features include fast acting RF inhibits triggered by electron emission probes in the input coupler, wave guide spark detectors, reflected power profiles that exceed prescribed limits and RF waveguide leaks.

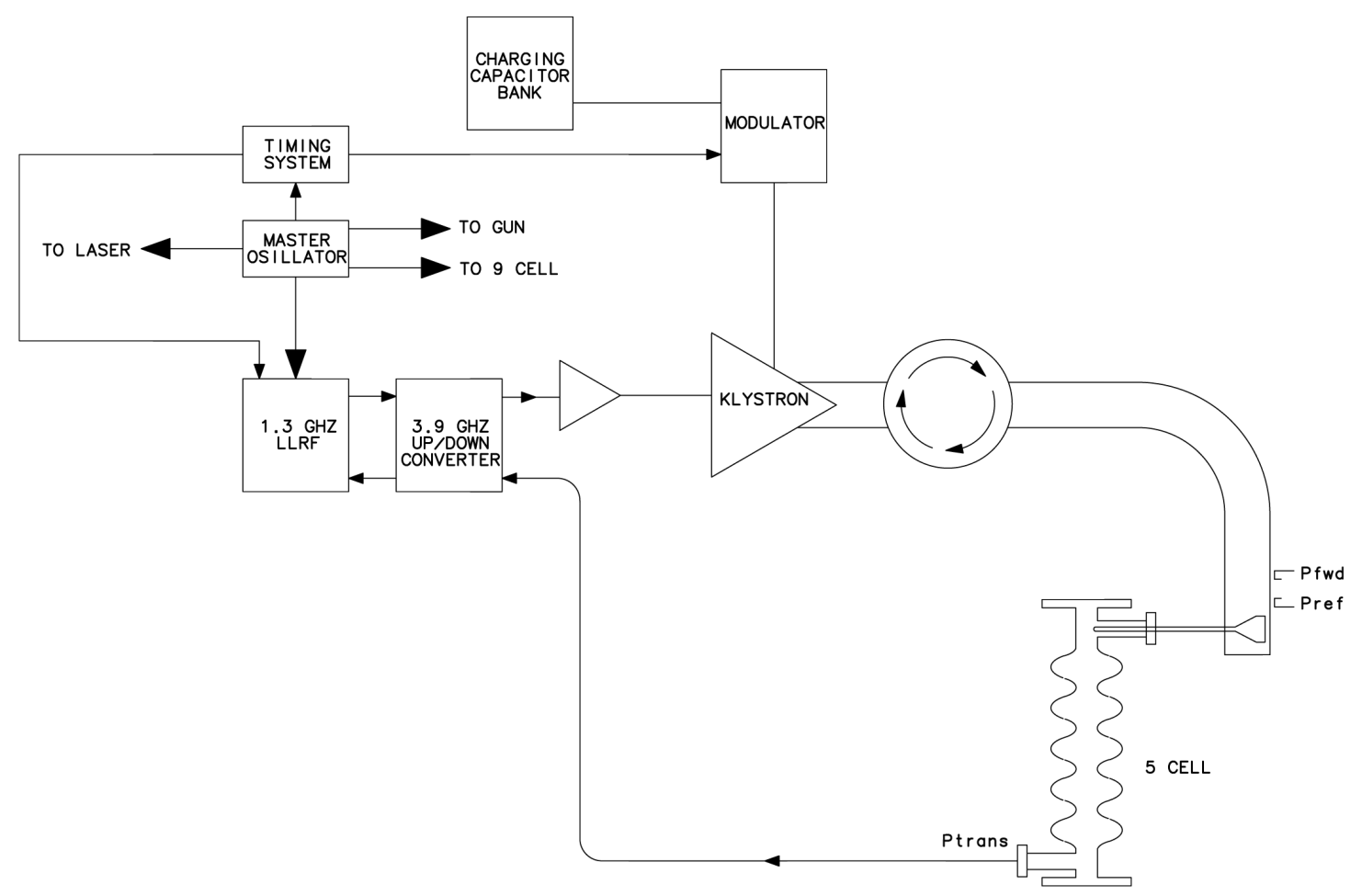

Figure 4.5: Overview diagram of $3.9 \mathrm{GHz}$ HLRF installation. 


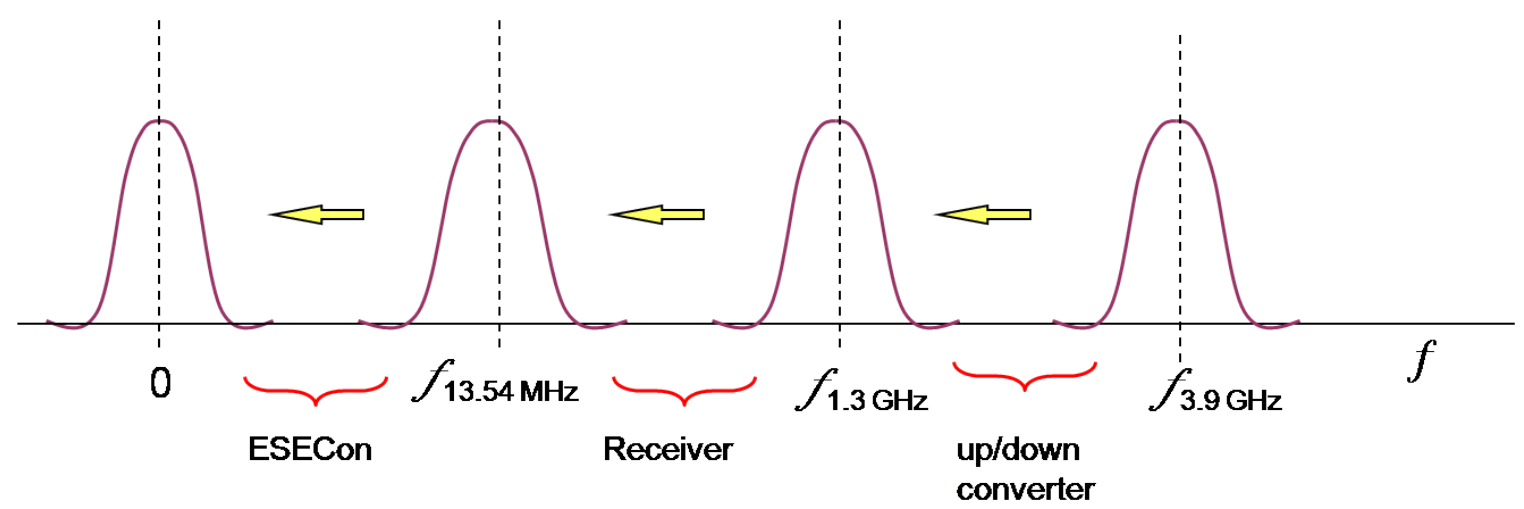

Figure 4.6: A total of three frequency down conversion stages bring the $3.9 \mathrm{GHz} T M_{110}$ Mode Cavity pickup probe signal to 'baseband' for regulation in the ESECON controller.

\subsection{2 $T M_{110}$ Mode Cavity Low Level RF Control}

Nominally the $T M_{110}$ Mode Cavity resonates at $3.9 \mathrm{GHz}$, however, during operation transient environmental effects detune the cavity. Phase, $\phi_{\text {cav }}$, frequency $\left(d \phi_{\text {cav }} / d t\right)$, and amplitude variations in the cavity fields occur, therefore we discuss an error 'bandwidth,' $\Delta f$, about $f_{R F}$. The $T M_{110}$ Mode Cavity gradient amplitude and phase information is sampled by the cavity's pickup probe and is used to determine the error signal. The regulation of our $T M_{110}$ Mode Cavity phase and amplitude is performed by two two stages of analog frequency conversion and a digital low level RF controller named ESECON.

ESECON is a Fermilab designed FPGA based general RF controller inspired from the DESY SimCon system used at TTF/Flash.[28] Its motivation was for application in the linac of a superconducting linear collider as well as in the Free Electron Laser. Since several versions of ESECON were already controlling superconducting $1.3 \mathrm{GHz}$ cavities, there was an analog $1.3 \mathrm{GHz}$ receiver-driver set associated with the ESECON controller. Thus, to make the existing hardware compatible with our $3.9 \mathrm{GHz} T M_{110}$ cavity, a 1.3 to $3.9 \mathrm{GHz}$ up/down converter was built. The frequencies of the LLRF down conversion stages are outlined in Figure 4.6.

The frequency conversion method known as mixing, or heterodyning, is utilized in both the analog and digital portions of the LLRF system. Mixing is the multiplication of two sinusoidal signals, $\omega_{1}$ and $\omega_{2}$, to produce intermediate frequencies $\omega_{1}+\omega_{2}$ and $\omega_{1}-\omega_{2}$; this can be seen from the trigonometric identity: 


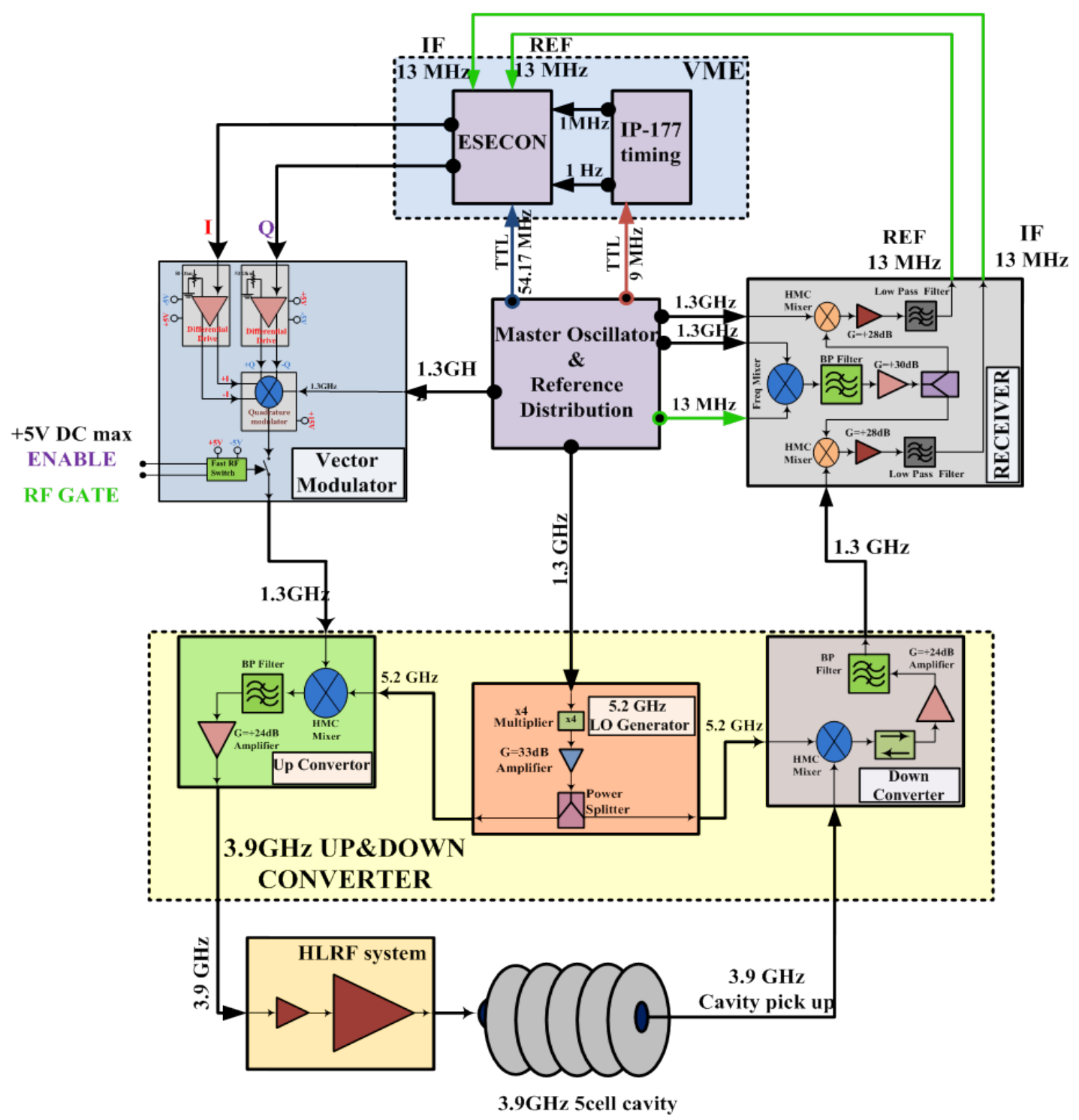

Figure 4.7: Block diagram of LLRF components. ESECON LLRF controller, analog updown conversion, and $1.3-3.9 \mathrm{GHz}$ up-down converter. 


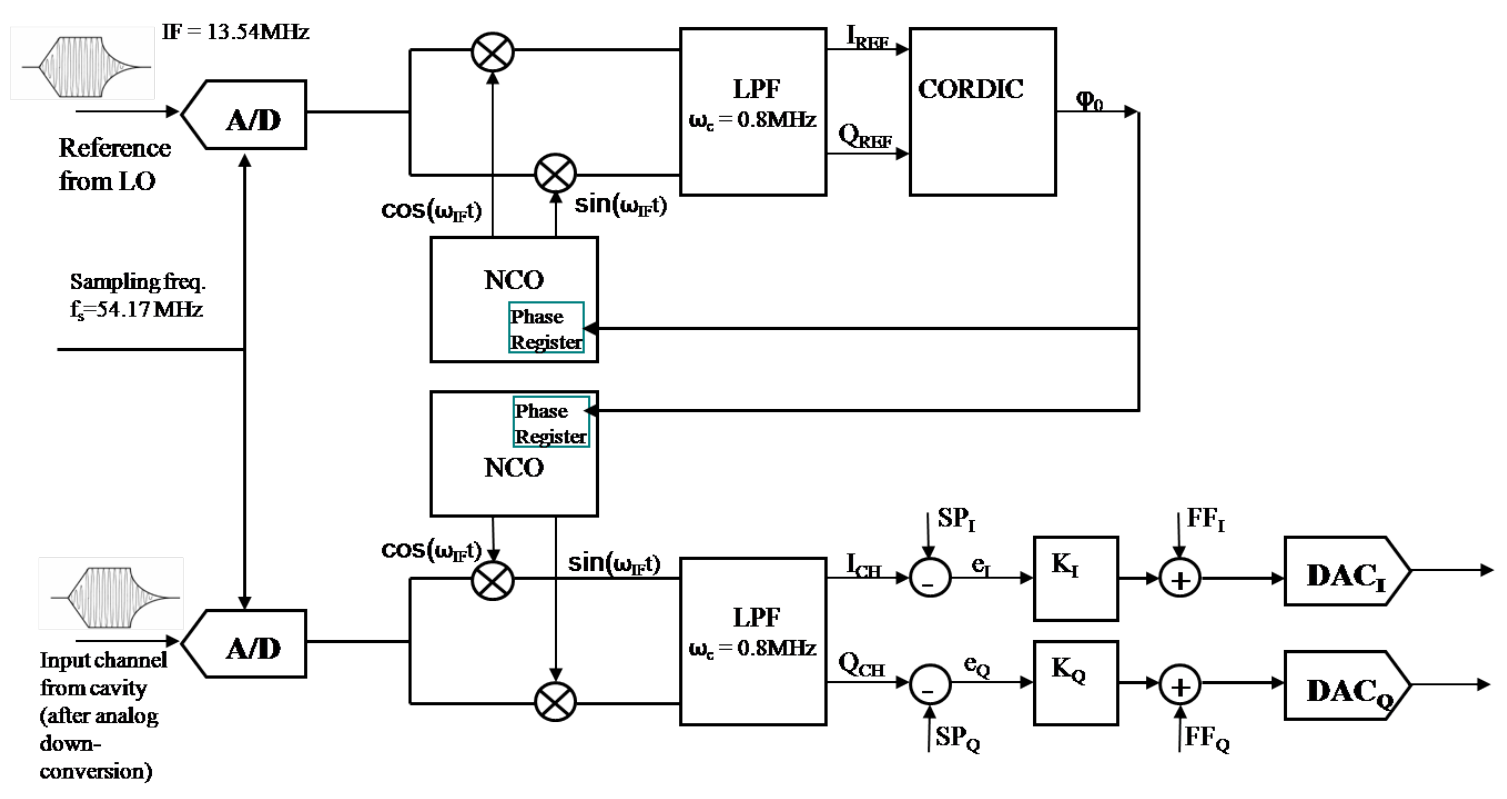

Figure 4.8: The ESECON Block Diagram

$$
\cos \left(\omega_{1} t\right) \cos \left(\omega_{2} t\right)=\frac{1}{2} \cos \left(\omega_{1} t+\omega_{2} t\right)+\frac{1}{2} \cos \left(\omega_{1} t-\omega_{2} t\right)
$$

Through the selection of a 'local oscillator' frequency, $f_{L O}$, the phase and amplitude characteristics of an $\mathrm{RF}$ signal, $f_{R F}$, can be shifted to intermediate frequencies, $f_{I F}=$ $f_{R F} \pm f_{L O}$. Therefore mixing can be used for both up and down conversion, and a filter can be used to remove the other, unwanted, $f_{I F}$. Figure 4.5 outlines the components of our 1.3$3.9 \mathrm{GHz}$ up/down converter, and their implementation between the $3.9 \mathrm{GHz} T M_{110}$ cavity and the analog section of $1.3 \mathrm{GHz}$ LLRF controller. A $1.3 \mathrm{GHz}$ master oscillator signal is quadrupled to $5.2 \mathrm{GHz}$ for the $f_{L O}$ and distributed to both the up and down converting sections. The $f_{L O}$ choice of $5.2 \mathrm{GHz}$ provided synchronization between the up and down channels yet avoided generation of intermodulation products. In the down converter, the mixed $5.2 \mathrm{GHz} f_{L O}$ and $3.9 \mathrm{GHz}$ cavity pickup probe signals are amplified and filtered to extract the $1.3 \mathrm{GHz}$ component. The $1.3 \mathrm{GHz}$ down converter output is feed into the analog receiver of the $1.3 \mathrm{GHz}$ controller. Conversely, the LLRF controller's $1.3 \mathrm{GHz}$ drive signal is mixed with the $5.2 \mathrm{GHz} f_{L O}$ and filtered to produce the $3.9 \mathrm{GHz}$ drive for HLRF system.

The function of the LLRF receiver is to provide the ESECON board both a downconverted $13.54 \mathrm{MHz}$ cavity probe signal and a phase stable $13.54 \mathrm{MHz}$ reference signal. 
The receivers components and signal flow are indicated in Figure 4.7. The receiver's 1.3 $\mathrm{GHz}$ down-conversions to $13.54 \mathrm{MHz}$ utilize a $1.313 \mathrm{GHz} f_{L O}$. The $1.31354 \mathrm{GHz} f_{L O}$ is locally generated in the receiver from mixing phase stable $13.54 \mathrm{MHz}$ and $1.3 \mathrm{GHz}$ signals from the master oscillator. Then the cavity's $1.3 \mathrm{GHz}$ pickup probe signal is mixed with the $1.31354 \mathrm{GHz} f_{L O}$ to generate the down converted $13.54 \mathrm{MHz}$ signal which is digitized by ESECON. An additional phase-stable master oscillator $1.3 \mathrm{GHz}$ 'reference' signal undergoes a parallel down conversion to $13.54 \mathrm{MHz}$ in a second receiver channel. The second 13.54 $\mathrm{MHz}$ down-converted signal is also digitized by ESECON and provides the reference phase signal against which the cavity pickup probe signal is compared.

The ESECON digitizes the pickup probe and reference $13.54 \mathrm{MHz}$ signals each with a 14-bit $\mathrm{ADC}$ at a sampling frequency, $f_{s}$ of $54.17 \mathrm{MHz}$. After the digitization both the cavity probe and reference signals are split to undergo a final down-conversion, digitally. The split $f_{R F}$ pairs are each mixed to 'baseband' with a $13.54 \mathrm{MHz} f_{I F}$ frequency generated from a digital numerically controlled oscillator (NCO). However, the two $f_{I F}$ are shifted by $90^{\circ}$, resulting in two orthogonal signals labeled I (in-phase) and Q (quadrature) which can be vectorially represented on the complex plane, as shown in Figure 4.9. Plotting the cavity's $\mathrm{I}_{\text {cav }}, \mathrm{Q}_{\text {cav }}$ on the complex plane, we see that a rotating vector can map out a circle of radius $A$. This procedure is know as quadrature de-modulation and is identically done for both the reference signal and the cavity probe signal. After the demodulation, all four signals, $\mathrm{I}_{c a v}, \mathrm{Q}_{c a v}, \mathrm{I}_{r e f}, \mathrm{Q}_{r e f}$ are low-pass filtered with a cutoff of $3 \mathrm{MHz}$. A phase detection algorithm, CORDIC, establishes a reference phase from $\mathrm{I}_{r e f}, \mathrm{Q}_{r e f}$. The reference phase is used in the digital phase lock loop for the reference NCO and synchronously locks the cavity pickup probe's NCO. With the phase reference signal we establish a $\phi_{o}$, inevitably named the 'reference phase,' with which to compare the cavity phase. In Figure 4.9 we define $\phi_{o}=0$, and for illustration we reconstruct the amplitude, A, and cavity phase offset:

$$
\begin{gathered}
A_{c a v}=\sqrt{I^{2}+Q^{2}} \\
\phi_{c a v}=\tan ^{-1}\left(\frac{Q}{I}\right)
\end{gathered}
$$




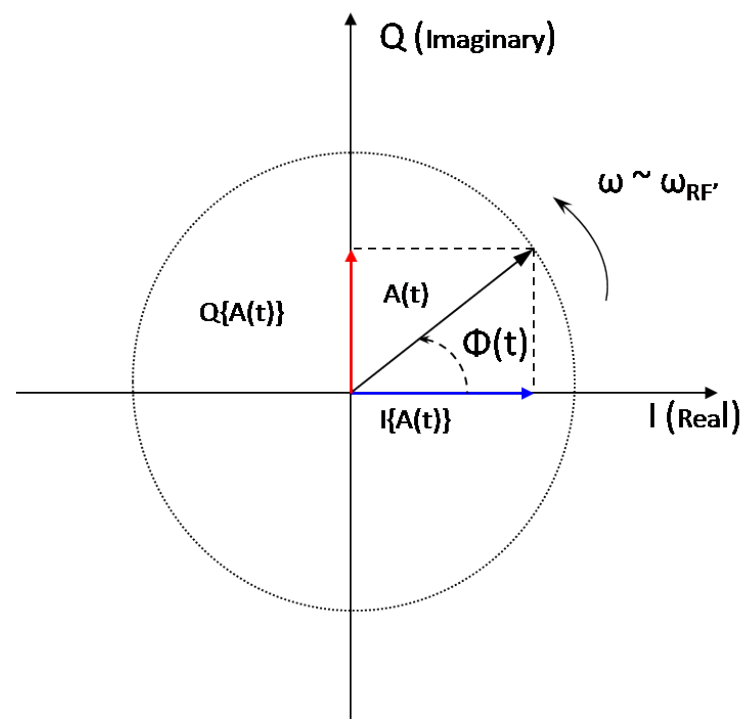

Figure 4.9: Cavity phase and amplitude representation in the complex plane by I \& Q vector decomposition.

Returning to the ESECON operation, after the $3 \mathrm{MHz}$ digital low pass filter, the $\mathrm{I}_{\text {cav }}, \mathrm{Q}_{\text {cav }}$ values are compared to setpoint values $\mathrm{SP}_{I}, \mathrm{SP}_{Q}$, their differences are the error signals. The error signals are amplified by Integral and Proportional Gain constants $\mathrm{K}_{I_{I}}$, $\mathrm{K}_{Q_{I}}, \mathrm{~K}_{I_{P}}, \mathrm{~K}_{Q_{P}}$ and summed with the programmed 'feed forward' tables, $\mathrm{FF}_{I}, \mathrm{FF}_{Q}$. The resulting signals are then returned to the analog realm by two 14-bit DACs.

A Proportional-Integral (PI) feedback algorithm is implemented. The proportional term adjusted the drive proportionally to the instantaneous error signal, while the integral term adjusts the drive signal according to the integrated error signal. Optimal control is achieved by the proper selection of these two parameters for minimum gradient and phase excursions.

The output analog I and Q signals drive an analog quadrature vector modulator which, using equations 4.5 and 4.6 impart amplitude and phase modulation to another synchronous phase stable $1.3 \mathrm{GHz}$ carrier. The modulated $1.3 \mathrm{GHz}$ signal is fed into the up conversion section of the 1.3-3.9 GHz up-down converter. The up converted $3.9 \mathrm{GHz}$ signal then drives the HLRF power amplifier chain which powers the $T M_{110}$ Mode Cavity gradient. Which completes the path through the $T M_{110}$ Mode Cavity RF loop. The combined performance of these systems (in an superconducting $3.9 \mathrm{GHz}$ cavity) have been measured: the rms jitter in gradient amplitude has been measured to be $2.5 \times 10^{-4}$ and an rms phase jitter of $0.5^{\circ}$ measured over a period of several minutes. 


\subsection{3 $T M_{110}$ Mode Cavity Operator Interface}

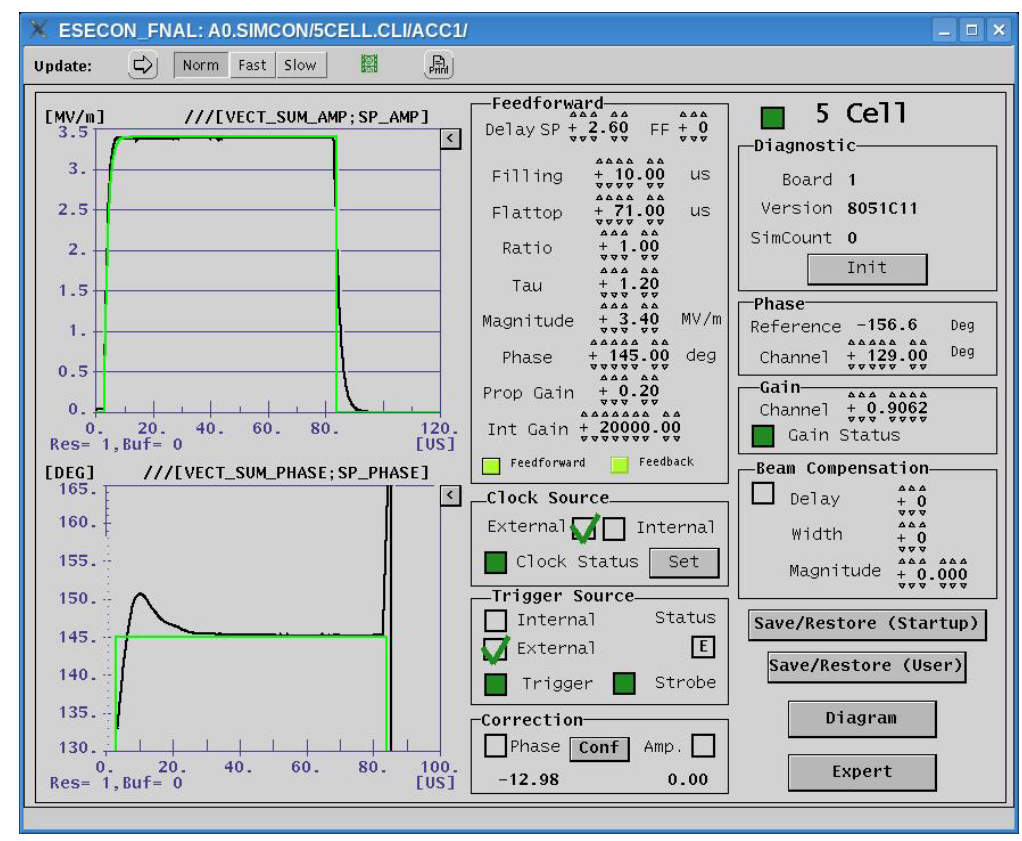

Figure 4.10: A screen shot of the typical operating parameters and response of the $T M_{110}$ Mode Cavity llrf operators interface.

The VME ESECON RF controller board is interfaced with a Linux based processor via the VME crate back plane bus. The processor has a small server that communicates with the ESECON's DAQ, memory and registers. This server can communicate with MatLab present on the Linux environment for development or with the DESY based control software, DOOCS, for control during beam operation.[28] A typical DOOCS operator's panel is displayed in Figure 4.10. 


\subsection{EEX Beamline: Apparatus \& Diagnostics}

Superficially, the A0 Photoinjector Emittance Exchange experimental apparatus consists of a $3.9 \mathrm{GHz} T M_{110}$ Mode Cavity located between two 'dogleg' magnetic channels. In actuality, the beam line is outfitted with a number of diagnostic devices to measure the electron beam parameters before, throughout and after the EEX beamline section. There are ten Beam Position Monitors (BPM) which provide transverse beam centroid position information. The transverse beam profiles can be measured by eight Optical Transition Radiation (OTR) viewing screens along the beamline. Two sets of tungsten slits and YAG viewing screen pairs measure the transverse beam divergence before and after the exchange beamline. EEX input and output central momenta and momentum spreads are measured by two spectrometer magnets and viewing screens. A single Hamamatsu C5680 streak camera outfitted with a synchroscan unit provides a measurement of the laser pulse length, electron bunch lengths at the input and output of the exchanger, and changes in bunch time-of-arrival at the picosecond level. Finally, a Martin-Puplett interferometer is installed at the end of the EEX beamline to perform sub-picosecond bunch length measurements.

This section will give a brief overview of the exchange apparatus and diagnostics, with the exception of the $T M_{110}$ Mode Cavity as Chapter 5 is reserved for a more thorough treatment of it. The reader is referred to Figure 4.11 for assistance in understanding the relative location of elements discussed.

\subsubsection{Bunch Charge Measurement}

Electron bunch charge can be measured by intercepting the beam with a retractable Faraday collector located just after the gun or with stationary Faraday collectors that form beam dumps at the end of each beamline. The Faraday cups are copper slugs with thickness about 1 inch [34] electrically separated from earth ground by a low resistance, $R$, typically $50 \Omega$. The voltage that develops across $R$ is brought into the control room via a coaxial cable and displayed and integrated by a digitizing oscilloscope. The resulting charge can be calculated from: 


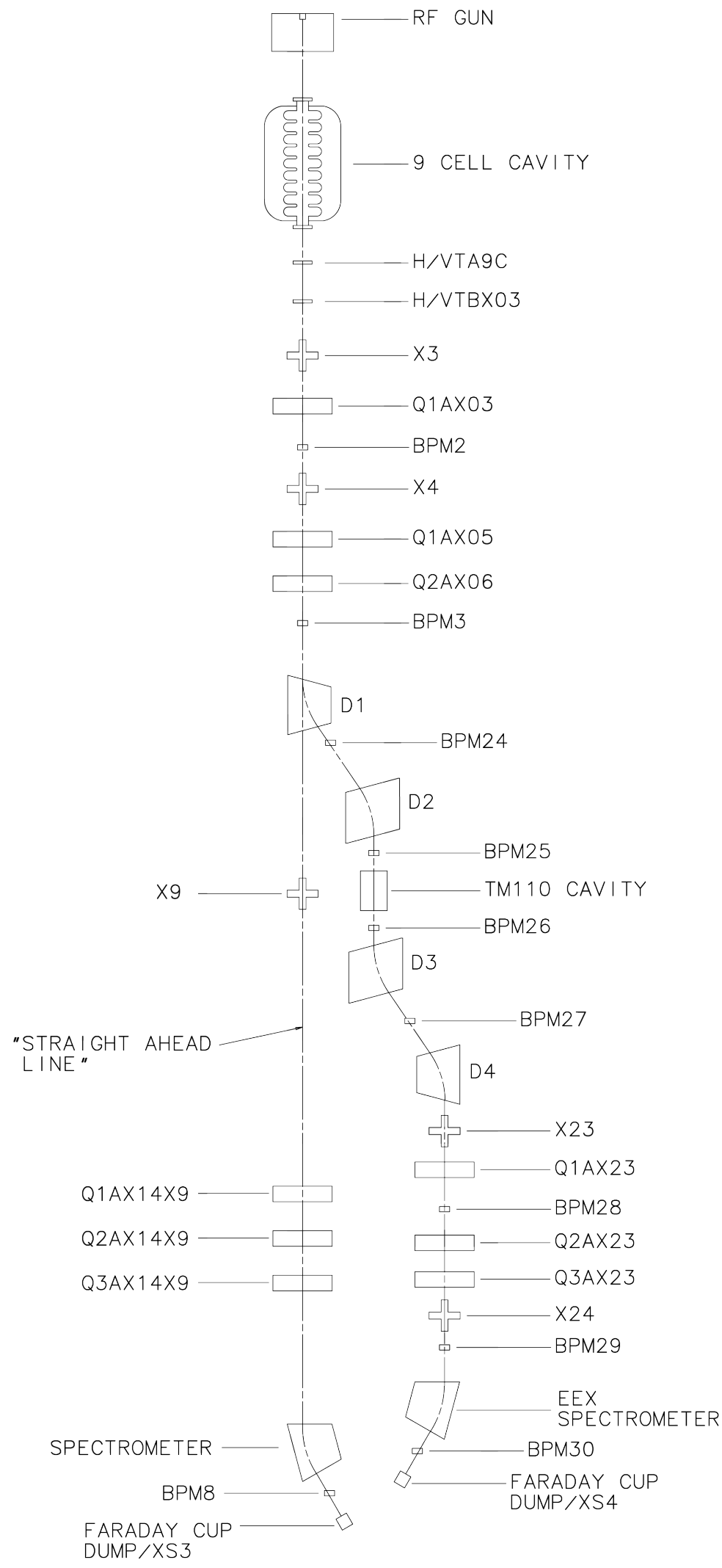

Figure 4.11: A schematic layout of pertinent beamline components and their relative positions (Not to scale) that will be heavily referred to during this chapter 

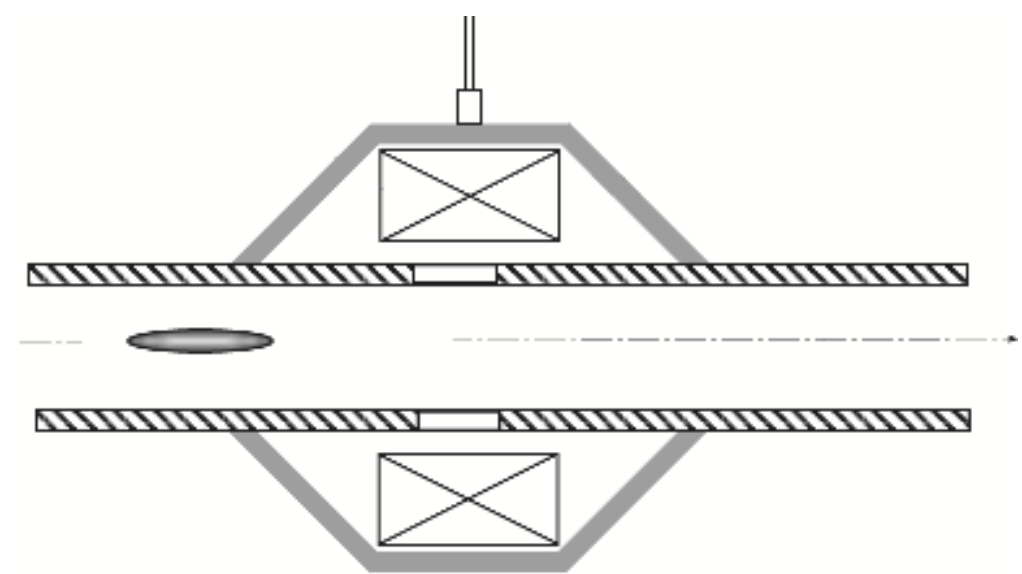

Figure 4.12: Installation of the Integrating Current Transformer (ICT)

$$
Q=\frac{1}{R} \int V(t) d t
$$

Additionally, a high frequency Integrating Current Transformer (ICT) provides a nondestructive method for measuring the bunch charge. The ICT, model ICT-122-070-20:1SMA, was purchased from Bergoz [42], and mounted outside of beam pipe over a short ceramic section forming a transformer of turns ratio of $N=20: 1$. A copper shroud, blanketing the ICT, maintains the electrical continuity of the beam pipe, as shown in Figure 4.12. The ICT's $50 \Omega$ termination is matched at the measuring oscilloscope with $50 \Omega$, thus the effective load is $25 \Omega$. The ICT's output $V(t)$ is integrated by the digitizing oscilloscope yielding the bunch charge as:

$$
Q=\frac{N}{R} \int V(t) d t=0.8 \int V(t) d t
$$

\subsubsection{Magnets of the Photoinjector}

The Photoinjector uses iron dominated magnets for deflection and focusing. Large dipoles are used to generate the dispersion and compression for the emittance exchange experiment, dispersion for the magnetic spectrometer, and small dipoles are used as for slight steering corrections throughout the beamline. Transverse focusing is accomplished by quadrupole magnets. Each magnet is independently controlled by current regulated 


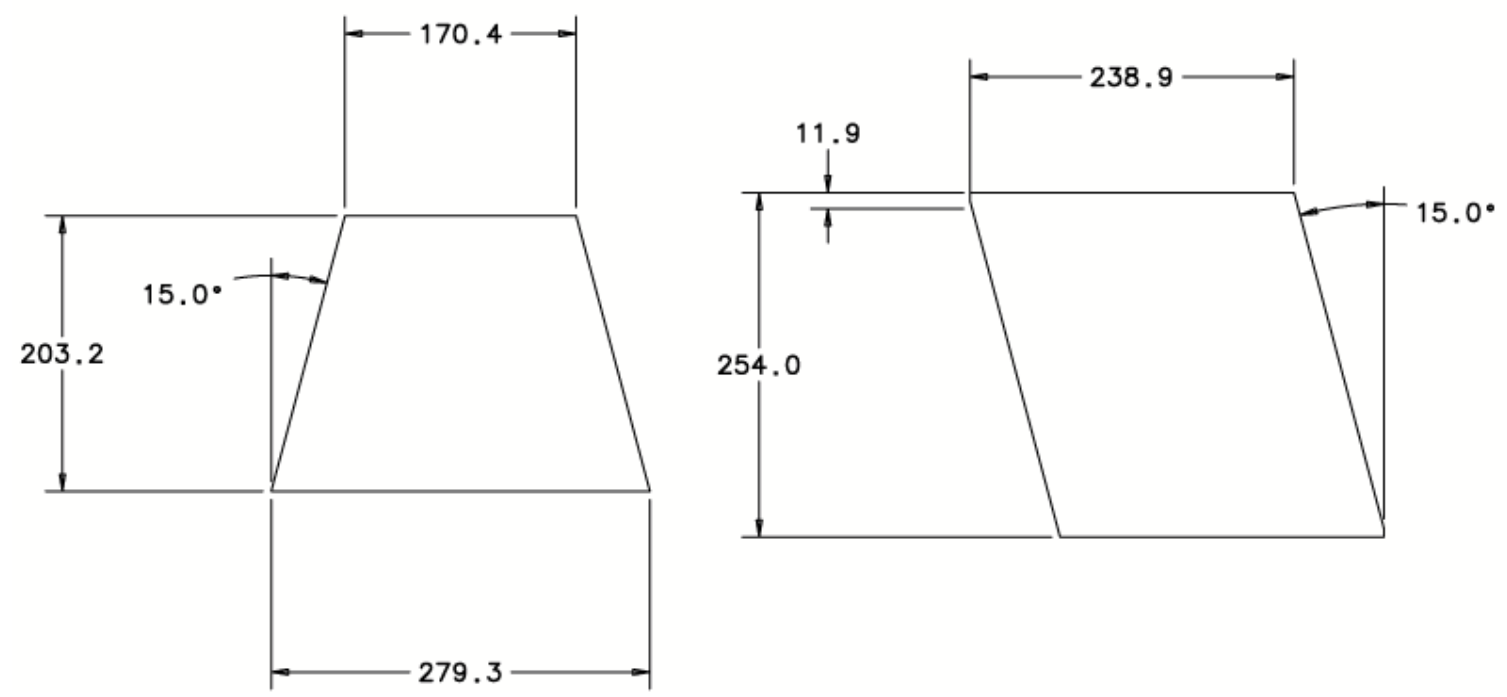

Figure 4.13: Dimensions of the parallelogram and trapezoidal pole tips

power supplies. Hysteresis is common to all of the magnets, thus for reproducibility are degaussed prior to beam operation.

\section{EEX Dipole Magnet}

The four dipole magnets that comprise the two magnetic doglegs have been recycled from a bunch compressor of a previous experiment. They are of C-frame construction making their insertion and removal without requiring a vacuum break possible. With exception of the two types of pole tips, trapezoidal and parallelogram, the magnets are identical in construction. The single, air cooled excitation coil is an 18 layer 87 turn coil wound about the vertical return yoke. They made use of edge focusing, hence the trapezoidal and parallelogram pole tip faces, to better equalize the horizontal and vertical focusing terms than that given by pure wedge magnet poles. The edge angles, outlined in Figure 4.14, were chosen such that the dispersion growth was zero, $D^{\prime}=0$, between Dipoles 2 and 3 , between which was the $T M_{110}$ Mode Cavity location. zero. In both pole tip cases the air gap is 53 $\mathrm{mm}$, the area of the parallelogram pole tips is $60,700 \mathrm{~mm}^{2}$ while the area of the trapezoidal poles tips is $45,700 \mathrm{~mm}^{2}$. The edge angles are summarized in table 4.2 using the CERN handbook [41] convention and an illustration defining the input and output edge angles is shown in Figure 2.1.

A measurement of the vertical (bending) component of the fringe field in the region 


\begin{tabular}{|c|c|c|}
\hline Dipole & Input Angle & Output Angle \\
\hline 1 & $15^{\circ}$ & $-7.5^{\circ}$ \\
\hline 2 & $7.5^{\circ}$ & $15^{\circ}$ \\
\hline 3 & $-15^{\circ}$ & $-7.5^{\circ}$ \\
\hline 4 & $7.5^{\circ}$ & $-15^{\circ}$ \\
\hline
\end{tabular}

Table 4.2: Main dipole edge angles.

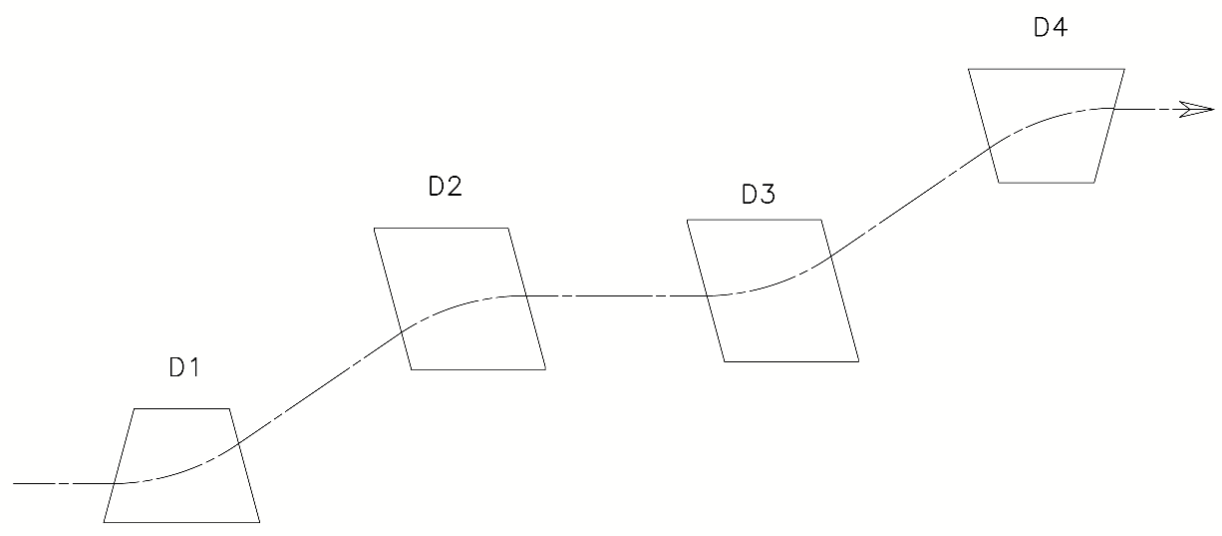

Figure 4.14: Top view of pole tip arrangement indicating edge angles

between the Dipole 1 and Dipole 2 is displayed in Figure 4.15. The investigation was prompted after current corrections to Dipole 1 were needed in order to keep the beam horizontally positioned at BPM24 after Dipole 2 was energized. Figure 4.15 indicates that the fringe fields are extending further than expected and are having a canceling effect. The edge of the magnet's physical poletips are depicted as solid black lines, while the $50 \%$ at $1 / 2$ the gap height soft edge approximation is indicated by the vertical green lines. In light of this finding, a spare of each magnet type is being assembled on the bench without the obstruction of a beam pipe for a thorough field mapping.

\section{Magnetic Spectrometers}

Both the straight ahead and emittance exchange beamlines are terminated by magnetic spectrometers to measure the beam's momentum and momentum spread. The two spectrometers are Fermilab NDA correction dipole magnets supplemented with trapezoidal pole tips [25]. A thorough analysis of the straight ahead beamline spectrometer was performed by Bishopberger [29]. An electron of momentum $p_{z}$ is bent through $45 \mathrm{deg}$ when the magnet is energized according to: 


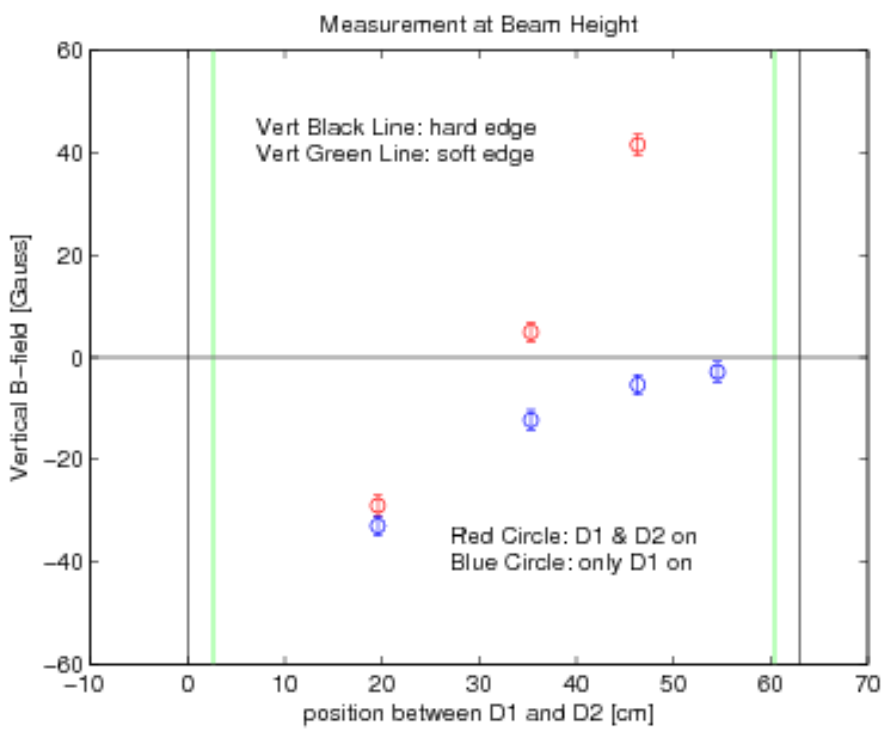

Figure 4.15: A measurement of the vertical magnetic field between Dipoles $1 \& 2$

$$
\begin{gathered}
p_{z}[M e V / c]=1.619 i[A] \\
D_{\text {spect }}=\left(\rho_{o}+L \tan \delta\right)(1-\cos \theta)+L \sin \Theta
\end{gathered}
$$

where $\rho_{o}$ is the bending radius, $\approx 370 \mathrm{~mm}$, the bending angle $\Theta$ is $45^{\circ}$, and the exit edge angle, $\delta$ is $22.5^{\circ}$. Thus the dispersion of the straight ahead viewing screen, located a distance $L=253 \mathrm{~mm}$, calculates to $318 \mathrm{~mm}$ and was measured to be $324 \pm 5 \mathrm{~mm}$. The dispersion at BPM8, located prior to the straight ahead viewing screen, was measured to be $240 \pm 5 \mathrm{~mm}$. Similarly, the vertical dispersion of the spectrometer in the EEX line was measured to be $940 \mathrm{~mm}$ at the the viewing screen and $865 \mathrm{~mm}$ at BPM30.

\section{Quadrupole Magnets}

Two versions of quadrupole magnets are found in the A0 Photoinjector, they slightly differ in construction and strength calibration. The types are distinguished by their painted color: blue and green, and placement is depicted in Figure 1.1. For a given energizing current, $i[A]$, strength of the green quadrupoles are $0.778\left[\frac{T}{m \cdot A}\right]$ while the blue quadrupoles are $0.461\left[\frac{T}{m \cdot A}\right]$. For both quadrupole types the physical longitudinal length is $86 \mathrm{~mm}$ while 
the effective length is $106 \mathrm{~mm} .[40]$.

To relate the quadrupole gradient to the strength, $\kappa$, of equations 2.9 and 2.10 we introduce the so-called magnetic rigidity $\mathbf{B} \rho$.

$$
B \rho=3.33 \times 10^{-3} \frac{p_{s}}{e}[M e V / c]
$$

where $p_{s}$ is the particle momentum $e$ is the electron charge, thus for $14.3 \mathrm{MeV}$ electrons is $0.048 \mathrm{~T} \cdot \mathrm{m} . \mathbf{B} \rho$ relates the quadrupole gradient to $\kappa$ by:

$$
\kappa=\frac{1}{B \rho} \frac{d B_{y}}{d x}
$$

\subsubsection{Vacuum System}

Because of the installation of a superconducting RF cavity, the quality requirements of vacuum and internal cleanliness at the A0 Photoinjector is very stringent. Gate valves

allow the beamline to be isolated into six separate volumes. All vacuum based beamline components are cleaned in ultrasonic baths with ultra-pure water in a class 1000 clean room. All subcomponents are blown with dry nitrogen until a particulate count of $10 \mu \mathrm{m}$ falls below background in a class 10 clean room. As much as possible, sub-components are assembled in the class 10 clean room. Final in-situ connections are made under a portable clean room. Once vacuum tight, each of the beam lines volumes are pumped with oil free mechanical backed turbomolecular pumps to pressures of $1 \times 10^{-7}$ Torr or better. Below $1 \times 10^{-7}$ Torr, the mechanical pumps are isolated and ion pumps are engaged. Beam operation commences once a pressure at the ion pumps reach a few $10^{-9}$ Torr or below.

\subsubsection{Beam Position Monitors}

The A0 Photoinjector straight ahead and EEX beam lines are outfitted with a total of 14 four-button type beam position monitors (BPM) pickup stations. The four buttons signals (up, down, left, and right) of an individual BPM station are combined through a network of coaxial delay lines, as shown in Figure 4.16 resulting in a time-separated pulse train on a single signal cable. Each combined BPM station signal is then read out by a 


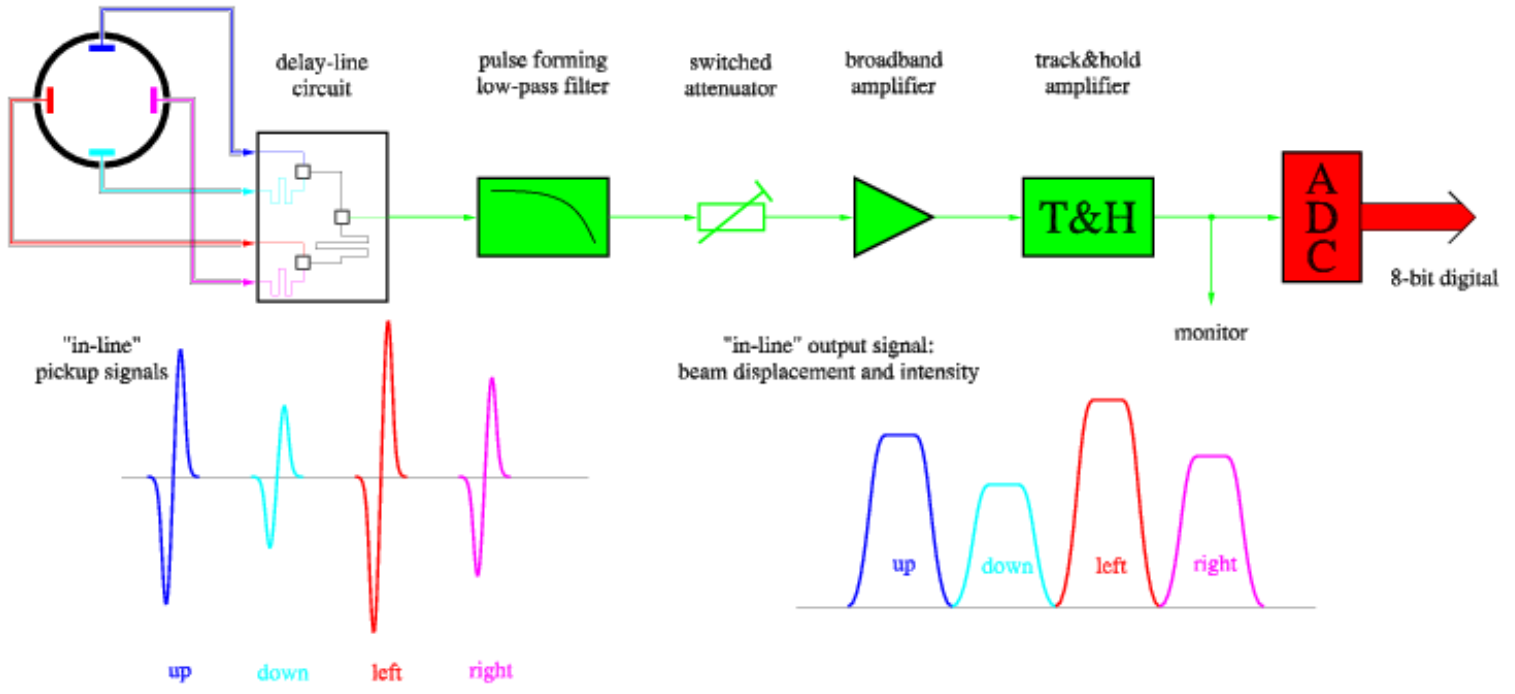

Figure 4.16: Schematic of a single BPM station system

single ADC. As the electron bunch passes through the interior of the BPM housing, offset from the electrical axis, the induced signals on the buttons are different. An absolute bunch centroid can then be deduced from the signal difference divided by the signal sum. 


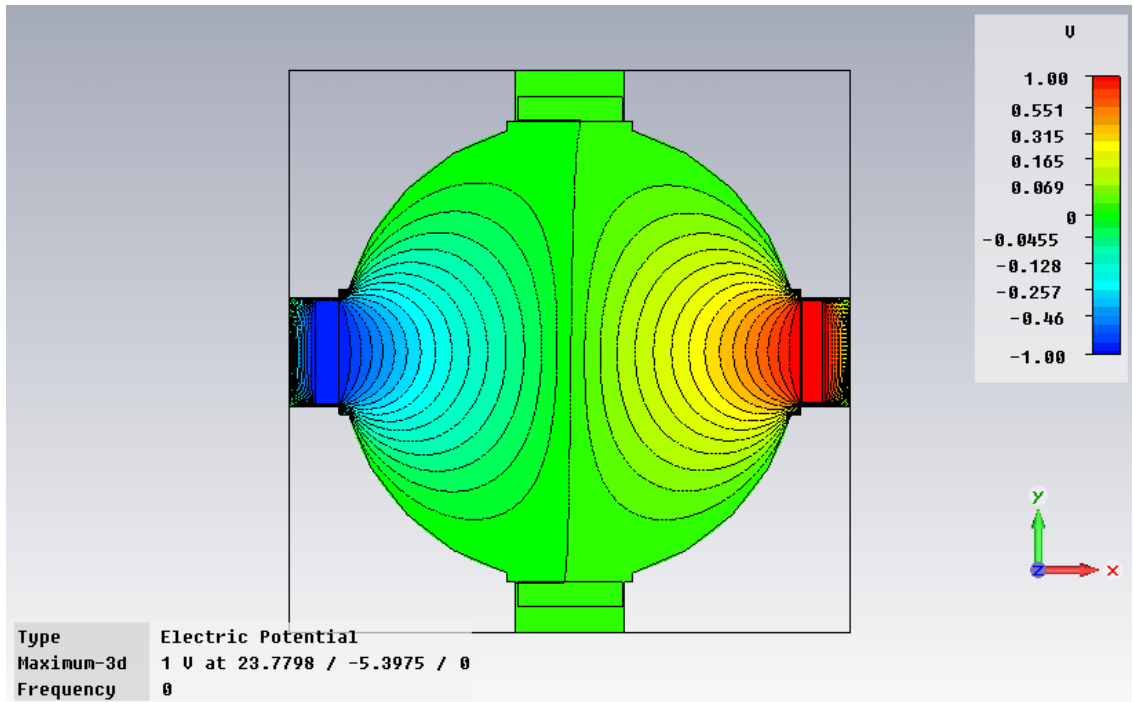

Figure 4.17: Equipotential maps between left and right (up and down) buttons for a two inch housing

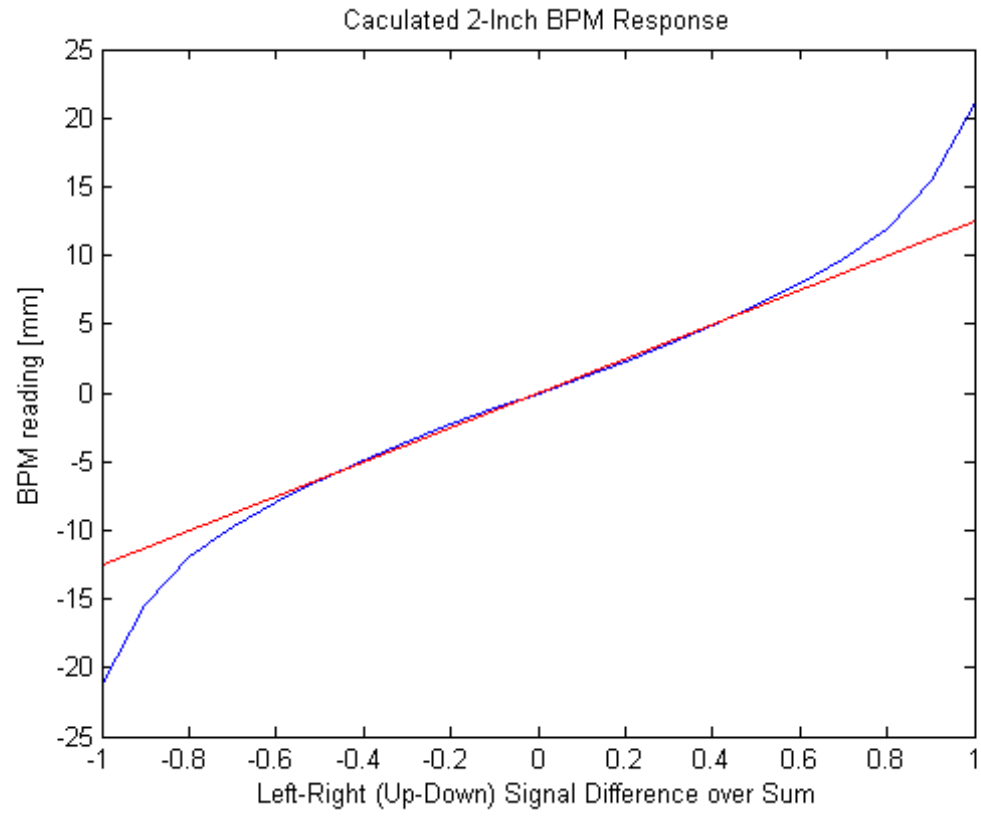

Figure 4.18: A linear fit (red line) to the linear region of a two inch BPM housing response (blue). 
A calculated equipotential map for a two inch BPM housing (BPM8 and BPM30 are four inch housings) is shown in Figure 4.17. A fit through the linear portion of the response yields the calibration constant of $12.54 \mathrm{~mm}$, as shown in Figure 4.18. There were insufficient ADC modules to dedicate an individual ADC channel to each of the 14 BPM stations. Thus, each of the BPM stations after the first EEX dipole share an ADC readout channel through yet another signal combiner. Since the beam propagation is either through the straight ahead line or the EEX line, the only inconvenience is a matter of bookkeeping, and is recorded in Appendix A. The BPM system only measures the centroid of the first bunch of a bunch train per RF pulse.

\subsubsection{Intercepting Viewing Screens}

Transverse beam profiles are destructively obtained by intercepting the beam with Optical Transition Radiation (OTR) screens and phosphor YAG:Ce $\left(\mathrm{Y}_{3} \mathrm{Al}_{5} \mathrm{O}_{12}\right.$ :Ce) screens. [35] In all cases, the viewing screens are placed at a $45^{\circ}$ angle with respect to the incident beam, and are viewed by Sony model XCD-X710 firewire based digital cameras. The cameras also view the screens at $45^{\circ}$, their axis forming $90^{\circ}$ with the beam, resulting in a 1:1 viewing projection ratio. The physical arrangement permits shielding the cameras within lead sarcophagi.

When an electron beam transitions between two media of differing indexes of refraction, transition radiation (TR) is produced. As the name suggests, Optical Transition Radiation is TR radiation in the visible spectrum. Our OTR screens are aluminum plated mirrors, thus transitioning between vacuum of permittivity $\varepsilon=1$ and a metal with permittivity $\varepsilon=\infty$. The electron beam is incident on and the OTR light viewed from the metallized surface of the mirror. The total spectral and spatial radiation energy per electron is then given by [34]:

$$
\frac{d^{2} U}{d \omega d \Omega}=\frac{r_{e} m_{e} c^{2}}{\pi^{2} c} \frac{\beta^{2} \sin ^{2}(\theta)}{\left(1-\beta^{2} \cos ^{2}(\theta)\right)^{2}}
$$

where $r_{e}$ is the radius of the electron, $m_{e}$ is the electron mass. Maximum emission occurs at $\theta=1 /(\beta \gamma)$. In our case $\gamma$ for $14.3 \mathrm{MeV}$ electrons is 28.4. 


\begin{tabular}{|c|c|c|}
\hline Screen & Spatial Resolution, $\sigma[\mu \mathbf{m}]$ & Temporal Response \\
\hline \hline Chromox $(0.25 \mathrm{~mm})$ & 200 & $300 \mathrm{~ms}$ \\
\hline YAG $(0.5 \mathrm{~mm})$ & $<100$ & $80 \mathrm{~ns}$ \\
\hline OTR & $<10$ & $10 \mathrm{fs}$ \\
\hline
\end{tabular}

Table 4.3: A comparison between Chromox, YAG, \& OTR viewing screens. It is interesting to note the large range of response times for the differing screens.

An experimental comparison of these three viewing materials was performed by A. Lumpkin et al. at the Argonne Advanced Photon Source.[36] with $600 \mathrm{MeV}$ electrons. The results are repeated in Table 4.3, and it is interesting to note the widely varying response times for the different screens. The benefit of the OTR light is its high resolution and prompt production and extinction, making temporal measurements of short $(<1 \mathrm{ps})$ high energy electron bunches possible.

\subsubsection{Slit Method of Transverse Emittance}

Our transverse emittances are measured using the 'slit' method, described in [43], and is schematically depicted in Figures 4.19 and 4.20. By means of a stepper motor actuator, the electron beam can be intercepted either by a viewing screen, or by either a set of vertical or horizonal $1 \mathrm{~mm}$ wide tungsten blocks separated by $50 \mu \mathrm{m}$, thus forming slits. The viewing screen yields the rms transverse spot size, $\sigma_{x, y}$, while the slits measure the uncorrelated beam divergence, $\sigma_{x^{\prime}, y^{\prime}}$. The slits admit only thin slivers of the beam which, after a drift $L$, are imaged on an OTR or more sensitive YAG screen. If the width of the slit images are significantly larger than $50 \mu \mathrm{m}$ slits width, it can be ignored. The width of each of the resulting striped images divided by the drift length yields the angular divergence, $\sigma_{x^{\prime}, y^{\prime}}$. The product of the rms spot size and the rms angular divergence is a measure of the unnormalized $\mathrm{rms}$ emittance, $\sigma_{x, y} \cdot \sigma_{x^{\prime}, y^{\prime}}=\epsilon_{x, y}$. 


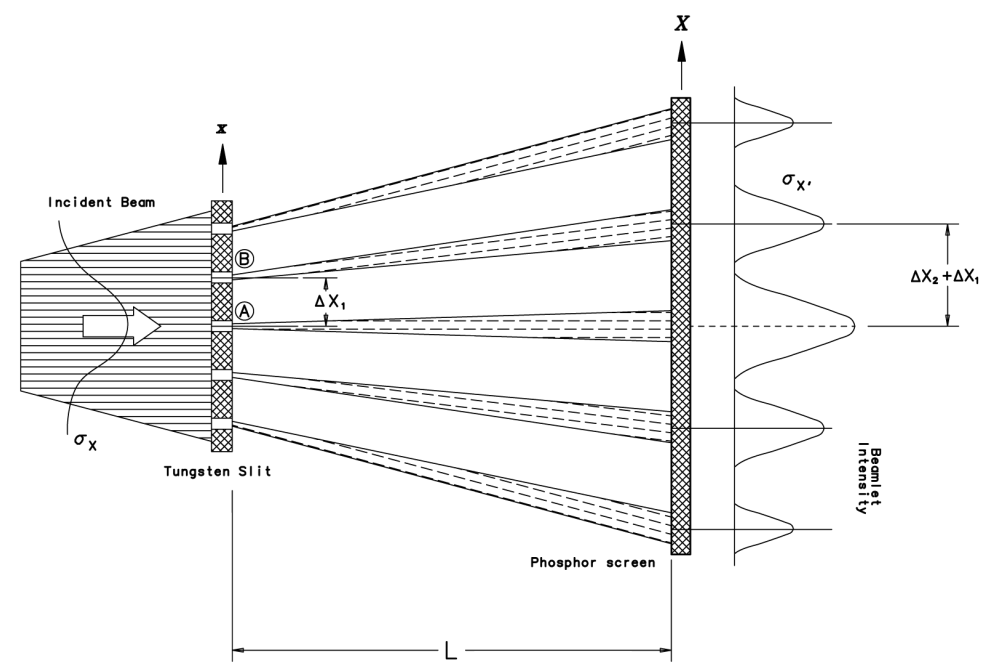

Figure 4.19: Slit method of measuring transverse emittances

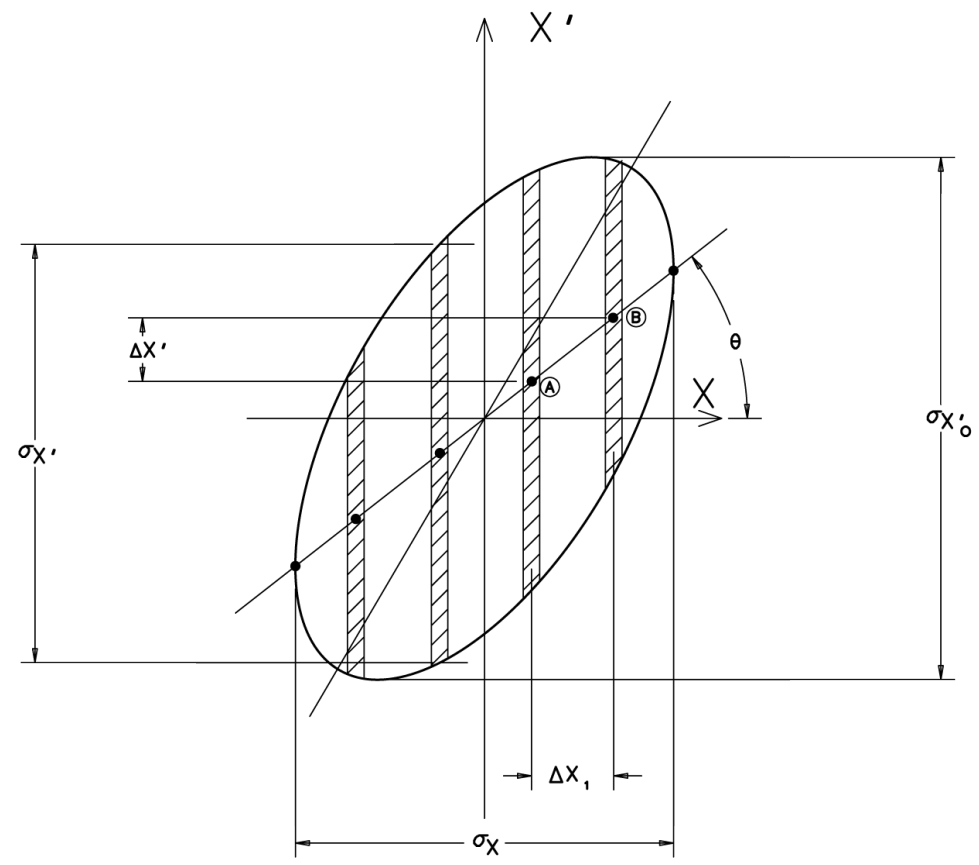

Figure 4.20: Slits emittance measurement yielding slice emittances, note markers A and B relating slits from above image. 
This slit technique has the benefit of not requiring the measurement to be located at a beam waist. Moreover, the spacing between the beamlet images compared to the spacing between the intercepting slits, indicates if the beam is converging, diverging, or at a focus. Figure 4.20 demonstrates that by measuring the spacing between beamlet, $\Delta X_{2}+\Delta X_{1}$, subtracting off the initial slit spacings, $\Delta X_{1}$, and dividing by the drift length, $L$, the change in angle, $\Delta X^{\prime}$, is calculated. Finally, the ratio of $\Delta X^{\prime} / \Delta X_{1}$ is the angle denoted as $\Theta$, which is equivalent to the ratio of Courant-Synder Parameters $-\alpha_{x, y} / \beta_{x, y}$. The $\beta_{x, y}$ can be calculated from the previous measurements of $\epsilon_{x, y}$ and $\sigma_{x, y}$, thus $\alpha_{x, y}$ can be determined. The individual beamlet images are samples, or slices, of the phase space through the bunch.

\subsubsection{Bunch Length Measurements}

\section{Streak Camera}

A Hamamatsu C5680 streak camera provides picosecond level measurement of the UV drive laser pulse length and electron bunch lengths at both the input and output of the exchanger. Our C5680 has recently been retrofitted with a synchroscan module allowing the streak camera to synchronously average low intensity, but repetitive, images.[47] The principle of operation of the streak camera is detailed in Figure 4.21. Incident photons, either from the UV drive laser or electron beam generated OTR screens, are optically transported to the streak camera input slit. Input optics focus the light onto the photocathode (4.21(A)) of the streak tube. A longitudinal static electric field accelerates the photoelectrons towards a multichannel plate $(\mathrm{MCP})$, while a transverse time varying electric field $(\mathrm{C})$ sweeps the photo electrons across the MCP. The MCP (D) increases the photoelectron current a thousand fold, and illuminates a phosphor screen (E). Thus, the position at which final electron signal impinges the phosphor screen is dependent on their generation time, or the initial image photons arrival time, as illustrated by two incident pulses of light in Figure 4.21. The bunch length is calculated by a LabView image capture and analysis program that operates a standard CCD camera which is focused on the streak camera's phosphor image screen.

Figure 4.22 is a screen shot of a typical digitized image and the on-line analysis software; raw images can be saved for further post processing. The profile of the CCD camera's image 


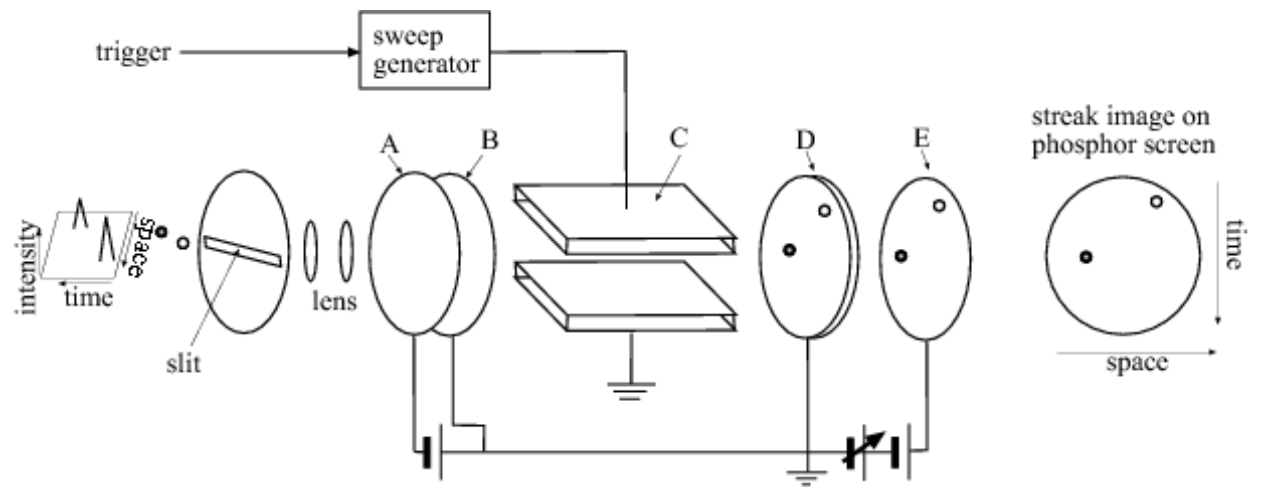

Figure 4.21: Principle of the streak camera operation

displayed in Figure 4.22 is the observed bunch length $\tau_{o b s}$, however, to obtain the actual bunch length, $\tau_{\text {act }}$ we must take into account the streak camera tube's resolution, $\tau_{r e s}$, the bandwidth $\left(\tau_{b w}\right)$ chromatic temporal dispersion effects from the broad band OTR screen, and the collective jitter effects of the electron beam, camera trigger signal, and laser jitter which are quadrature summed into one term, $\tau_{j}$. All of these uncorrelated contribution to $\tau_{o b s}$ are added in quadrature:

$$
\tau_{\text {obs }}^{2}=\tau_{\text {act }}^{2}+\tau_{\text {res }}^{2}+\tau_{b w}^{2}+\tau_{j}^{2}
$$

Therefore the actual bunch length is calculated by:

$$
\tau_{a c t}=\sqrt{\tau_{o b s}^{2}-\tau_{r e s}^{2}-\tau_{b w}^{2}-\tau_{j}^{2}}
$$

An investigation by A. Lumpkin, J. Ruan, and R. Thurman-Keup [44] into the performance of our streak camera details the measurement of the limiting factors, their uncertainties, and the calculation of the actual bunch length. Their findings applied to the EEX measurements are summarize in Table 4.4. As can be seen in Table $4.4 \tau_{j}$ is comparatively small and can be neglected. The dominant uncertainty is from the chromatic temporal dispersion. However, this term is not present when measuring the monochromatic UV laser pulse. Figure 4.24 gives the percent errors due to dispersion and resolution as a function of the corrected $\tau_{\text {act }}$ bunch length. 


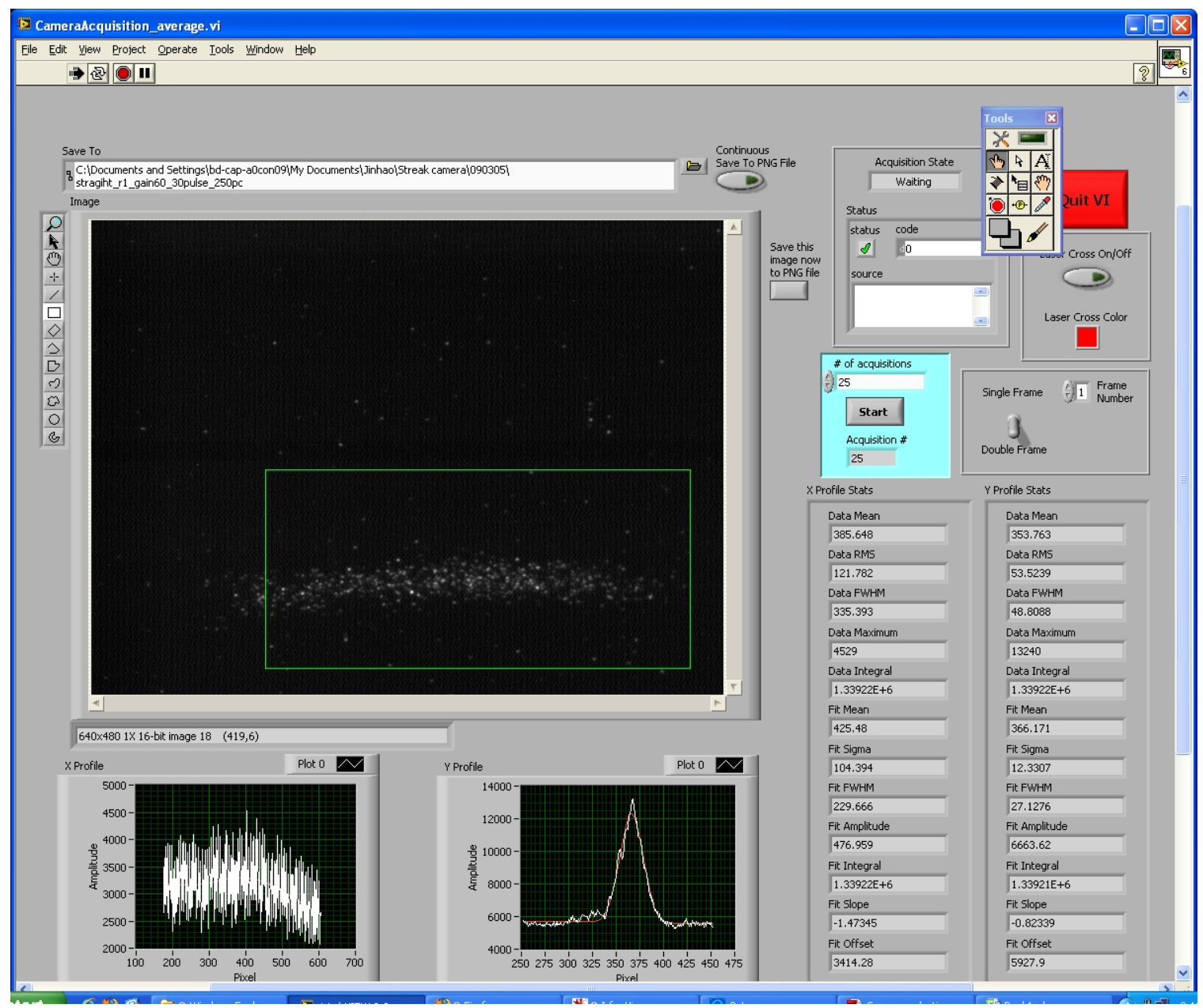

Figure 4.22: Streak camera image shown within LabView control and online analysis interface.

\section{Martin-Puplett Interferometer}

As describes above, transition radiation is generated by an electron beam transitioning between two regions of differing indexes of refraction. In our case, this is from a vacuum to a metal. Of the full spectrum of Transition Radiation (TR) generated, those wavelengths approximately the same as or greater than the electron bunch length are considered to be coherent. That is, the separation of head and the tail of the electron bunch is small compared to the TR wavelength, such that the radiated TR power is coherently enhanced. Obviously then, the shorter the bunch length the shorter the wavelength at which the coherent spectrum begins. For the case of our EEX experiment, where electron bunch lengths of order $300 \mu \mathrm{m}$ are anticipated, the peak CTR spectrum occurs in the infrared. A 


\begin{tabular}{|c|c|}
\hline Parameter & Contribution [rms ps] \\
\hline$\tau_{r e s}$ & $0.64 \pm 0.03$ \\
\hline$\tau_{b w}$ & $1.12 \pm 0.11$ \\
\hline$\tau_{j}$ & $<0.2$ \\
\hline
\end{tabular}

Table 4.4: Streak Camera Resolution limiting factors.

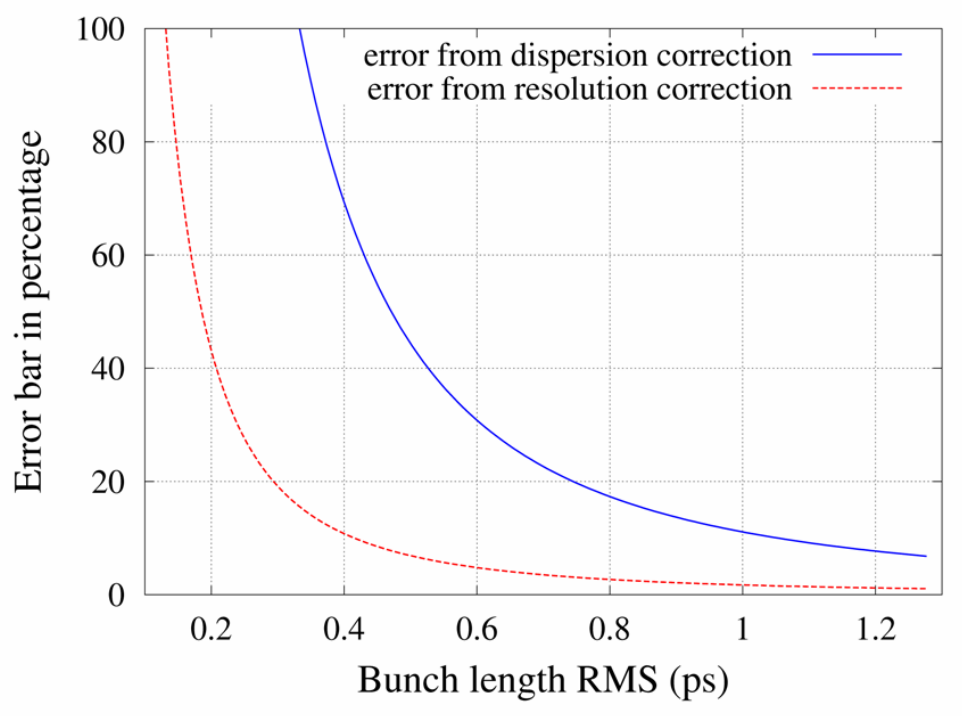

Figure 4.23: Calculation comparing contributions of $\tau_{r e s}$ and $\tau_{b w}$ to uncertainty of $\tau_{a c t}$ in percentage.

Martin-Puplett interferometer is used to measured the CTR spectrum, from which a Fourier decomposition is used to reconstruct the bunch length.

The Martin-Puplett interferometer, outlined in Figure 4.24, is a polarizing type interferometer that is used to measure the CTR spectrum, from which the electron bunch length can be calculated.[45, 46] The polarizing beam splitter consists of a wire grid with $15 \mu \mathrm{m}$ wires every $45 \mu \mathrm{m}$ and produces orthogonal signals at the two detecting elements. The difference in the two detectors is the interference signal and the sum is the incoming intensity and is used to normalize the measurement. From this, the magnitude and part of the phase of the spectrum generated by the bunch can be obtained. However, because of the finite size of the TR screen, vacuum view port aperture, and frequency response of the detecting elements, wavelengths longer than $100 \mathrm{GHz}$ are cut from the spectrum and have to be inferred. The missing part of the phase and the poor low frequency response of typical detecting elements cause unreliable measurements at bunch lengths above 1-2 ps. 


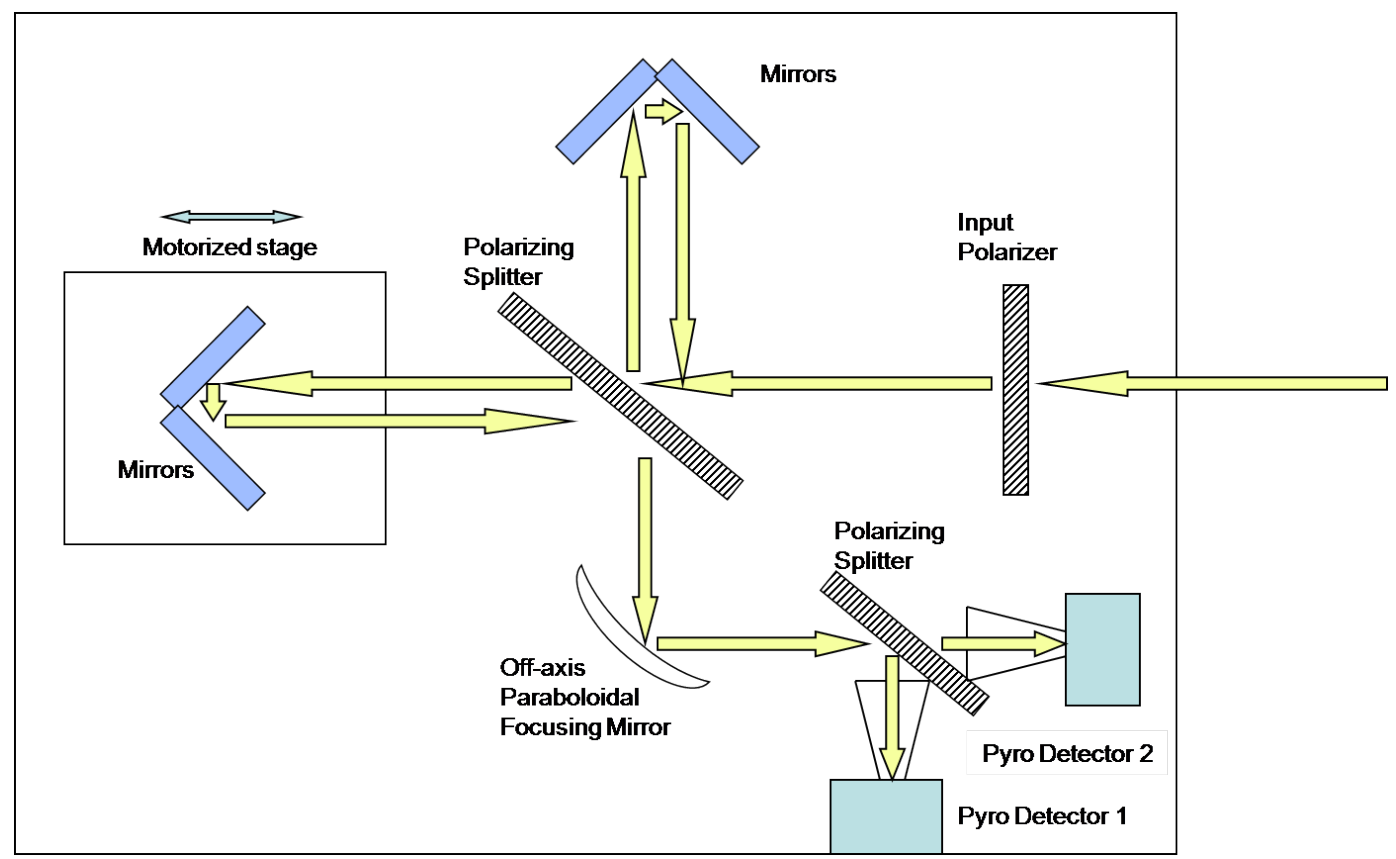

Figure 4.24: Interferometer Operation

The intensity is only a function of the magnitude of $F(\omega)$, the Fourier transform of the longitudinal bunch profile, and since the actual charge distribution is unknown, it is approximated to be gaussian.

The intensity of the CTR is given by:

$$
I(\omega)=I_{o}(\omega) \cdot\left(N+N(N-1)|F(\omega)|^{2}\right)
$$

where $I_{o}(\omega)$ is the spectrum from a single particle, and $N$ is the number of particles, and the complex factor $F(\omega)$ the the Fourier Transform of the longitudinal charge distribution:

$$
F(\omega)=\frac{1}{Q} \int \rho(z) e^{-i \omega z} d z
$$

We will review the operation of the interferometer. The CTR light exits the beamline through a quartz window and is directed to the interferometer through an optical transport of mirrors. The light incident on the interferometer enters through a polarizer resulting in a horizontally polarized wave.

$$
E(t)=E_{o} \sin (\omega t) n_{h}
$$


The wave is split by a diagonally positioned beam splitter creating two polarizations:

$$
\begin{aligned}
& E_{1}(t)=\frac{E_{o}}{2} \sin (\omega t)\left(n_{h}+n_{v}\right) \\
& E_{2}(t)=\frac{E_{o}}{2} \sin (\omega t)\left(n_{h}-n_{v}\right)
\end{aligned}
$$

which travel the length of the interferometer arms and arrive back at the splitter with a phase difference dependent on the path difference

$$
\begin{gathered}
E_{1}(t)=\frac{E_{o}}{2} \sin (\omega t-\omega \tau)\left(n_{h}+n_{v}\right) \\
E_{2}(t)=\frac{E_{o}}{2} \sin (\omega t)\left(n_{h}-n_{v}\right)
\end{gathered}
$$

The path length of one of the interferometer's arms is varied by a computer controlled motorized stage. The 'roof' mirrors rotate the polarization such that the initially reflected wave is then transmitted, and conversely, the initial transmitted wave is then reflected, such that the two are then recombined:

$$
E(t, \tau)=\frac{E_{o}}{2}\left[(\sin (\omega t-\omega \tau)+\sin (\omega \tau)) n_{h}+(\sin (\omega t-\omega \tau)-\sin (\omega \tau)) n_{v}\right]
$$

The recombined wave is then passed through another splitter directing the two polarizations to their respective pyroelectric sensors. The average intensity detected by each pyroelectric sensor is

$$
\begin{aligned}
& I_{h}(\omega, \tau) \propto \frac{E_{o}^{2}}{2} \cos ^{2}\left(\frac{\omega \tau}{2}\right) \\
& I_{v}(\omega, \tau) \propto \frac{E_{o}^{2}}{2} \sin ^{2}\left(\frac{\omega \tau}{2}\right)
\end{aligned}
$$

The interferogram of a given frequency, $\omega$, is generated by the difference of the intensities 
divided by their sum.

$$
S(\omega, \tau)=\frac{I_{h}-I_{v}}{I_{h}+I_{v}}=\cos (\omega \tau)
$$

The measured intensities are integrals over all frequencies:

$$
S(\tau)=\frac{\int I(\omega) \cos (\omega) d \omega}{\int I(\omega) d \omega}
$$

From which the frequency spectrum can be reconstructed from the real part, $\Re$ of the inverse Fourier transform, $F^{-1}$ :

$$
I(\omega)=\left|\Re\left[F^{-1}\{S(\tau)\}\right]\right|
$$

Finally, the longitudinal bunch profile is reconstructed from:

$$
\rho(t) \propto F^{-1}\left\{|\sqrt{I(\omega)}| e^{\left.i \frac{2 \omega}{\pi} \int_{0}^{\infty} d \omega^{\prime} \frac{\ln \frac{|\sqrt{I(\omega)}|}{\mid \sqrt{I(\omega) \mid}}}{\omega^{2}-\omega^{\prime 2}}\right\}}\right.
$$

Examples of the collected data are shown in Figure 4.25. The inferred data of the cutoff longer wavelengths is noted in the middle plot of Figure 4.25. Two scenarios are considered, an 'optimistic' and 'pessimistic' calculation of the spectrum and the reconstruction of the missing longer wavelengths. The rms bunch length profiles are then calculated for both scenarios. We report the mean value between the two scenarios, and their uncertainties to be the difference between the 'optimistic' and 'pessimistic' cases. 

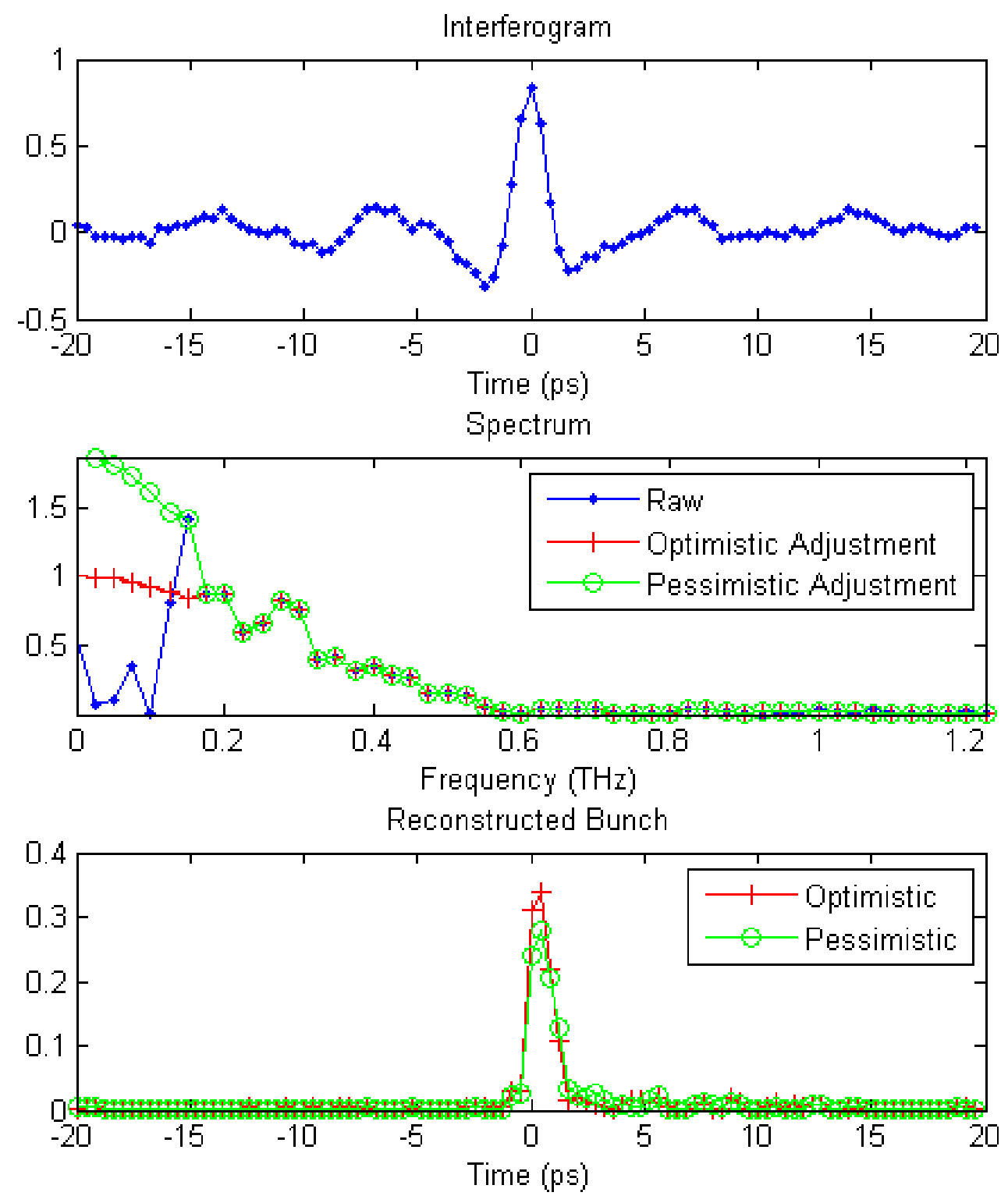

Figure 4.25: Example M-P Interferometer Analysis: First an interferogram (upper), the inferred spectrum including optimistic and pessimistic reconstruction of the missing longer wavelengths (middle), and the calculated bunch lengths of both optimistic and pessimistic spectrums (lower). The uncertainties assigned are then the variation between the optimistic and pessimistic values of bunch length. 


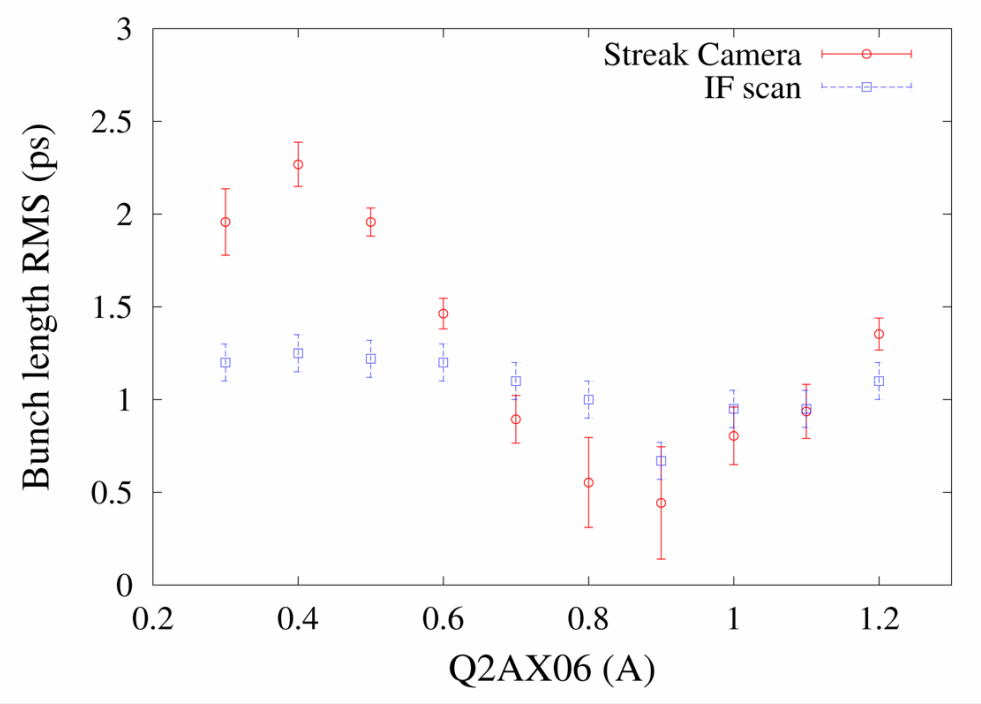

Figure 4.26: A comparison between the Interferometer and Streak Camera using short bunches produced by the EEX beamline. The bunch length was varied by scanning input quadrupole Q2AX06.

\section{Streak Camera-Interferometer Comparison}

Utilizing the short bunch lengths that are generated from the EEX beamline, a side by side comparison of the streak camera and interferometer was performed.[44] It is clear that within uncertainties the streak camera and interferometer are agreement for bunch lengths shorter than $\approx 1 \mathrm{ps} r m s$, however the streak cameras uncertainties become large for the short bunch lengths. Greater than $\approx 1$ ps rms the streak cameras uncertainties become practical, while the long wavelength cut-off effects become pronounced in the interferometer. Therefore the streak camera is reserved for bunch length measurements on order of 1 ps rms or greater, while the M-P interferometer is put into service for bunch lengths on order of 1 ps rms or less. 


\section{Chapter 5 The $T M_{110}$ Mode Cavity}

Exploiting high voltage build up in resonant radio frequency (RF) circuits for the acceleration of charged particles dates back to the dawn of accelerator physics itself. Nearly a century later resonant RF cavities are not only the engines of accelerators but have found application in the intricate manipulations of high energy particle beams. The success of this thesis owes itself to the utilization of the unique electric and magnetic fields delivered by the $T M_{110}$ Mode Cavity .

\subsection{Cavity Basics}

A simple model of a standing wave cavity resonator is a volume whose boundaries are distanced such that reflections between the boundaries develop standing waves at discrete [resonant] frequencies. The amplitudes of the multiply-reflected waves, electrical, mechanical, or otherwise, constructively interfere; resulting in amplitudes far exceeding the amplitude of the driving input. This amplitude build up at resonant frequencies is what fuels majestic baroque pipe organs and has empowered a millennia of children to hear the voice of the sea in conch shells. Figure 5.1 illustrates a gentleman scientist observing an enhanced [resonant] tone selected from a spectrum of background noise [51]. The integrated time averaged energy of these waves over the resonator's volume is the stored energy. In any non-ideal system, a portion of the stored energy will be lost to the resonator's walls; in the electrical case, power is dissipated from induced currents on the cavity's resistive walls. A resonator's figure of merit is its quality factor, $Q$, defined as the ratio of stored energy to the dissipated energy per radian: 


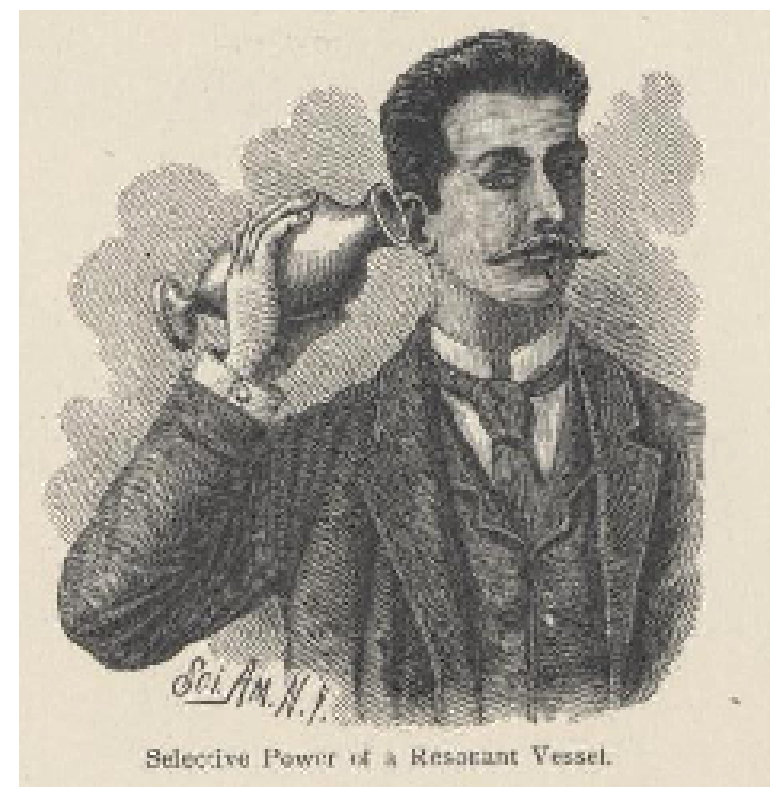

Figure 5.1: The magic of resonance as illustrated from this 1929 Sci. Am. Article

$$
Q \equiv \frac{2 \pi f U}{\left\langle P_{\text {dis }}\right\rangle}
$$

A consequence of non-ideal boundaries is the ability of the resonator to host a spectrum of frequencies about a resonant frequency. The width of this spectrum is inversely related to the quality factor; the so-called full-width at half-maximum (FWHM) bandwidth is given by:

$$
\frac{\Delta f_{F W H M}}{f} \equiv \frac{1}{Q}
$$

A completely sealed cavity resonator has a natural $Q$, denoted as $Q_{o}$, however inputting power at the resonant frequencies requires that a coupling port puncture the sealed cavity wall. Such a port is a reciprocal device, meaning that both energy can be supplied and drained from the resonators volume. Then, in addition to the resistive losses of the cavity walls, the coupling port presents itself as another energy sink with an external quality factor $Q_{e}$. The combined loaded quality factor, written as $Q_{L}$ is the reciprocal sum of $Q_{o}$ and $Q_{e}$ :

$$
\frac{1}{Q_{L}}=\frac{1}{Q_{e}}+\frac{1}{Q_{o}}
$$


Any coupling port will similarly contribute to the final $Q_{L}$ reciprocal sum, however, in the case of our $T M_{110}$ Mode Cavity, the only significant $Q_{e}$ encountered is the main power input coupler. The $Q_{e}$ of the pickup probe is insignificant and is neglected.

The coupling coefficient, $\beta$, is define as the ratio of $Q_{o}$ to $Q_{e}$ :

$$
\beta=\frac{Q_{o}}{Q_{e}}
$$

It can be easily shown that maximum power transfer occurs for $\beta=1$, unity coupling.

Using Equation 5.1, the $Q_{o}$ of a resonator can be determined from the ratio of the stored energy:

$$
U_{\text {total }}=\frac{1}{2} \mu_{o} \int_{V}|H|^{2} d v=\frac{1}{2} \epsilon_{o} \int_{V}|E|^{2} d v
$$

to the power dissipated, $P_{d i s s}$, by the resonator's walls:

$$
P_{\text {diss }}=\frac{1}{2} R_{s} \int_{s}|H|^{2} d s
$$

so,

$$
Q_{o}=\frac{2 \pi f_{o} \mu_{o} \int_{V}|H|^{2} d v}{R_{s} \int_{s}|H|^{2} d s}
$$

where $\mu_{o}$ is the permeability of vacuum and $R_{S}$ is the resonator's uniform surface resistance calculated by:

$$
R_{s}=\sqrt{\frac{\pi f \mu_{o}}{\sigma}}=\frac{1}{\sigma \delta_{\text {skin }}}
$$

where $\sigma$ is the conductivity of the wall material. The right most part of Equation 5.8 relates $R_{s}$ to the familiar microwave skin-depth $\delta_{\text {skin }}$. The task of determining $Q_{o}$ and other electrical parameters for resonators of complicated geometry is left to cavity design codes, such as Poisson SuperFish [52], MAFIA [53], and HFSS [54].

Presently, the application of superconducting materials to resonant RF structures, with $Q_{o} \approx 10^{10}$, is the state of the art in high gradient and high intensity accelerators, details 


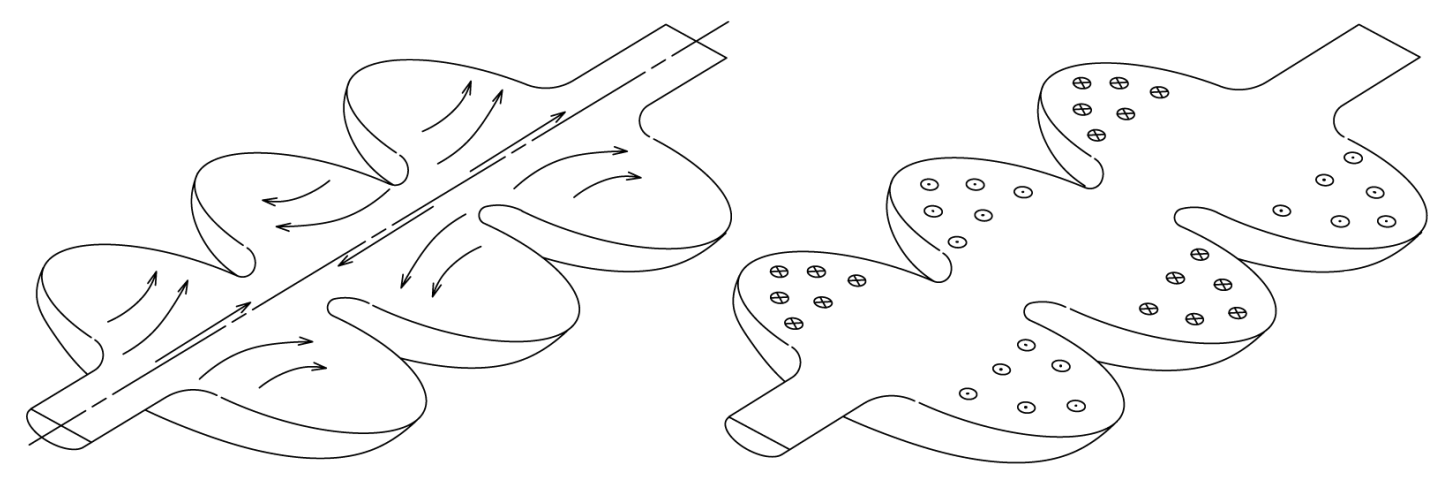

(a)

(b)

Figure 5.2: A conceptual drawing of the $\mathrm{E}(\mathrm{a})$ and $\mathrm{B}(\mathrm{b})$ fields of an 'accelerating,' $T M_{010}$, $\pi$ mode cavity.

can be found in references such as [55], [56], and [57].

\section{$5.2 T M_{010} \& T M_{110}$ Mode Cavity Field Descriptions}

Exemplified by this chapter's title, a resonant cavity's electric and magnetic fields of the 'mode of vibration' are often described by the resonating mode's transverse field type, transverse magnetic $-T M$, or transverse electric $-T E$, followed by three indices, $m, n, p$. The indices decompose the fields into the three orthogonal spatial vectors. The subscript $m$ indicates the number of complete rotations of the azimuthal components, subscript $n$ is the number less one of half periods in the radial direction of the axial components, and finally $p$ is the number of longitudinal half periods less one.

In the simplest case we consider a single cell pillbox resonator - a cylinder of radius $R$ and length $l$, whose volume is bounded by perfectly conducting walls. We do not consider the perturbation introduce by beam pipes. In cylindrical coordinates the wave equation is $[56]:$

$$
\frac{\partial^{2} E_{z}}{\partial z^{2}}+\frac{1}{r} \frac{\partial E_{z}}{\partial r}+\frac{\partial^{2} E_{z}}{\partial r^{2}}-\frac{1}{c^{2}} \frac{\partial E_{z}^{2}}{\partial t^{2}}=0
$$

Solutions for $E_{z}, E_{r}, E_{\phi}, B_{z}, B_{r}$, and $B_{\phi}$ for the complete set of pillbox $T M_{m, n, p}$ modes can be found in accelerator texts such as [55]. For illustration, we will evaluate only two. The 
most familiar solution is for the $T M_{010}$ mode cavity, where the strong axial component, $E_{z}$ is used for acceleration, Figure 5.2. This mode is typically called the 'accelerating mode.'

$$
\begin{aligned}
& E_{z}=E_{o} J_{o}\left(\frac{2.405 r}{R}\right) \cos (\omega t) \\
& B_{\phi}=\frac{E_{0}}{Z_{o}} J_{o}\left(\frac{2.405 r}{R}\right) \sin (\omega t)
\end{aligned}
$$

where $R$ is the radius of the pillbox, $r$ is the radial displacement from the axis, $J_{0}, J_{1}$ are zeroth- and first-order Bessel functions respectively, and $Z_{o}=377 \Omega$ is the impedance of free space. The $T M_{010}$ mode is the lowest resonant frequency mode of the pillbox; the frequency is:

$$
\omega_{010}=\frac{2.405 c}{R}
$$

It is interesting to note that the $T M_{010}$ cavity's frequency is independent of the longitudinal length, and only depends on the radius. Next, for $m=1$, the resulting dipole $T M_{110}$ mode, the fields for the pillbox cavity are [16]:

$$
\begin{gathered}
E_{z}=E_{o} J_{1}\left(\frac{k_{1} r}{R}\right) \cos \phi \cos (\omega t) \\
E_{r}=0 \\
E_{\phi}=0 \\
B_{z}=0 \\
B_{r}=E_{0} \frac{\omega R^{2}}{Z_{o} c k_{1}^{2} r} J_{1}\left(\frac{k_{1} r}{R}\right) \sin \phi \sin (\omega t)
\end{gathered}
$$




$$
B_{\phi}=E_{0} \frac{\omega R}{Z_{o} c k_{1}} J_{1}^{\prime}\left(\frac{k_{1} r}{R}\right) \cos \phi \sin (\omega t)
$$

where $k_{1}=3.832$ is the first root of the $J_{1}(x)$ Bessel function, and $R$ is the cavity radius. These fields earn the names 'dipole mode' or 'deflecting mode' due to their transverse deflection of particles traveling parallel to the axis. The combined vertical magnetic field and longitudinal transverse electric field gradient $E_{z}^{\prime} \equiv \frac{d E_{z}}{d x} \neq 0$ of this $T M_{110}$ structure imparts a transverse force to axially traversing particles. This is the general statement of the Panofsky-Wenzel Theorem. [58]. For those more familiar with accelerating mode cavities than deflecting mode cavities, we will develop the Panofsky-Wenzel theorem in the next subsection.

First we will approximate the fields near the central region and on the horizontal, $x$, plane, $\phi=0$, of the pillbox resonator. Equations 5.13 through 5.15 approximate to:

$$
\begin{gathered}
E_{z} \approx E_{o} \frac{x}{R} \cos (\omega t) \\
E_{r}=E_{\phi}=0
\end{gathered}
$$

Then by applying Maxwell's equation, $\vec{\nabla} \times \vec{E}=-\frac{\partial \vec{B}}{\partial t}$, to 5.19 we see that:

$$
\begin{gathered}
B_{y}=\frac{E_{o}}{R \omega} \sin (\omega t) \\
B_{x}=B_{z}=0
\end{gathered}
$$

Thus it can be seen that an electron traversing the cavity a distance $x$ from the axis $(|x| \ll$ $R$ ) will experience a fractional energy change of:

$$
\delta \approx x \frac{e V_{o}}{E \rho} \cos (\omega t) \approx k x
$$

where $E$ is the beam energy, $V_{o}$ is the integrated electric field at a reference radius $\rho$ constrained to the region where $E_{z}^{\prime} \equiv \frac{d E_{z}}{d x}$ is assumed linear, and $k$ is the cavity strength. 


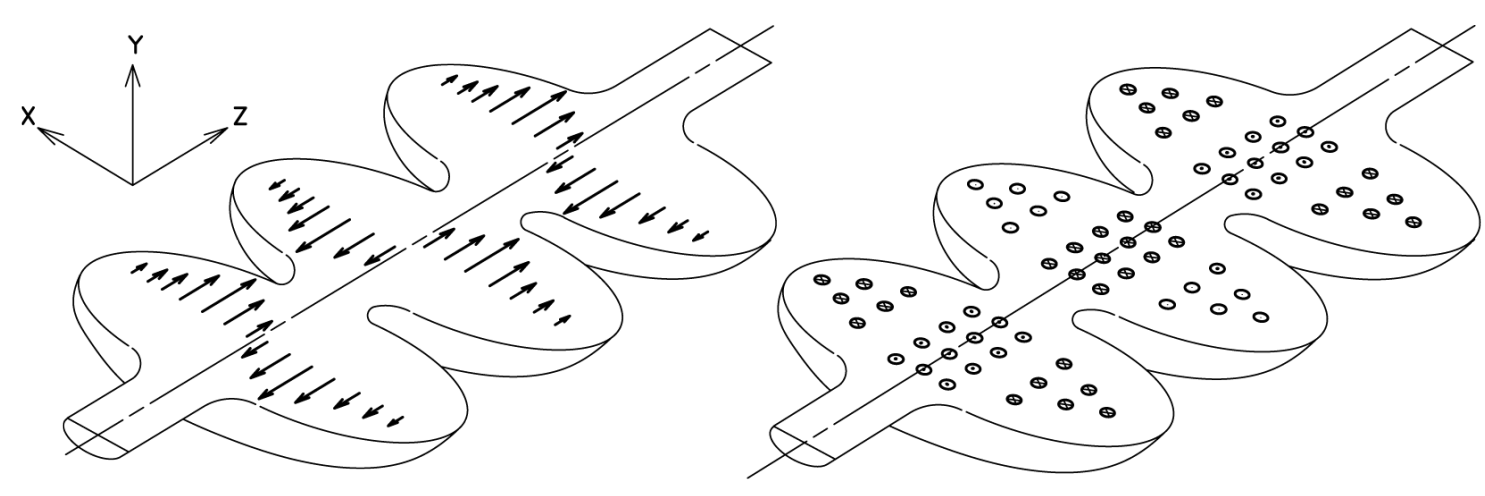

(a)

(b)

Figure 5.3: A conceptual drawing of the $\mathrm{E}(\mathrm{a})$ and $\mathrm{B}(\mathrm{b})$ fields of a deflecting, $T M_{110}, \pi$ mode cavity.

Similarly, by taking $\sin (\omega t) \approx \omega t$ near $t=0$ we can see that a particle entering slightly before or after the synchronous (crossing the center of the cavity, $z=0$, at $t=0$ ) particle will experience a transverse deflection, $\Delta x^{\prime}$ :

$$
\Delta x^{\prime}=\frac{e V_{o}}{E \rho} \frac{1}{\omega} \sin (\omega t) \approx \frac{e V_{o}}{E \rho} \Delta z=k \Delta z
$$

These are the results used to derive the thin lens cavity transport matrix 3.5.

\subsubsection{The Panofsky-Wenzel Theorem}

The Panofsky-Wenzel Theorem [58] calculates the transverse momentum change, $\Delta p_{\perp}$, for a particle of charge $q$ and velocity $v$ traveling longitudinally through an RF field of a cavity of length $d$. The following development of the Panofsky-Wenzel theorem follows the treatment by T. Wangler given in his text on RF Linear Accelerators.[56]. Assume the particle is parallel to the cavity axis and does not undergo a significant transverse displacement while inside the cavity. The change in transverse momentum is a result of the combined time dependent $E$ and $B$ fields, which are typically not in phase. Thus the change in transverse momentum is approximately:

$$
\Delta p_{\perp}=\frac{q \int_{z=0}^{d}\left[E_{\perp}+(v \times B)_{\perp}\right] d z}{v}
$$


We can describe the cavity's electric and magnetic fields in terms of the vector potential, $\vec{A}:$

$$
\vec{E}=-\frac{\partial \vec{A}}{\partial t}, \quad \vec{B}=\vec{\nabla} \times \vec{A}
$$

Defining $\nabla_{\perp}=\vec{\nabla}-\frac{\partial}{\partial z} \hat{z}$ we write:

$$
(v \times B)_{\perp}=[\vec{\nabla}(\vec{v} \cdot \vec{A})-(\vec{v} \cdot \vec{\nabla}) \vec{A}]_{\perp}=\nabla_{\perp}(\vec{v} \cdot \vec{A})-(\vec{v} \cdot \vec{\nabla}) A_{\perp}
$$

Substituting Equations 5.26 and 5.27 and taking $v_{z}$ to be constant we can write:

$$
\Delta p_{\perp}=q \int_{z=0}^{d}\left[-\left(\frac{1}{v} \frac{\partial A_{\perp}}{\partial t}+\frac{\partial A_{\perp}}{\partial z}\right)+\nabla_{\perp} A_{z}\right] d z
$$

Taking the total derivative of $A_{\perp}$ :

$$
\frac{d A_{\perp}}{d z}=\left(\frac{1}{v} \frac{\partial A_{\perp}}{\partial t}+\frac{\partial A_{\perp}}{\partial z}\right)
$$

and substituting into Equation 5.28 we can write:

$$
\Delta p_{\perp}=-q \int_{A_{\perp(z=0)}}^{A_{\perp(z=d)}} d A_{\perp}+q \int_{0}^{d} \nabla_{\perp} A_{z} d z
$$

Taking the Electric field of Equation 5.26 to harmonically oscillate with frequency $\omega, A=$ $e^{i \omega t}$ now allows us to write:

$$
\Delta p_{\perp}=-q \frac{i q}{\omega} \int_{A_{\perp(z=0)}}^{A_{\perp(z=d)}} d E_{\perp}+\frac{i q}{\omega} q \int_{0}^{d} \nabla_{\perp} E_{z} d z
$$

In either the pillbox cavity with perfectly conducting end walls or in a real world cavity with sufficiently long beam pipes, $E_{\perp}$ becomes zero, thus the first term of Equation 5.31 becomes zero. This leaves us with the remarkable result that the total transverse momentum kick, $\Delta p_{\perp}$ can be ascertained from just the transverse gradient of the longitudinal electric field.

$$
\Delta p_{\perp}=\frac{i q}{\omega} \int_{0}^{d} \nabla_{\perp} E_{z} d z
$$


The Panofsky-Wenzel theorem is most popularly used to evaluate the degree of [undesired] transverse momentum kick incurred in real-world accelerating cavities. A non-zero $\nabla_{\perp} E_{z}$ occurs off-axis in accelerating mode cavities, as can be seen in Figure 5.2; and obviously, a large value of $\nabla_{\perp} E_{z}$ occurs in the appropriately named $T M_{110}$ deflecting mode cavity, as seen in Figure 5.3.

\section{3 $T M_{110}$ Mode Cavity Overview and Requirements}

In Chapter 3 it was shown that in order to satisfy the EEX condition for our beamline a $T M_{110}$ Mode Cavity was required to provide a 'shear' gradient whose integrated longitudinal electric field at a reference of $1 \mathrm{~mm}$ off axis was $45 \mathrm{kV}, k_{o}=\frac{-1}{D}$, where $D$ is the dispersion, of $330 \mathrm{~mm}$, at the $T M_{110}$ Mode Cavity location. The efforts of Fermilab's superconducting RF program had brought a $3.9 \mathrm{GHz}$ superconducting $T M_{110}$ Mode Cavity to a prototype level $[63,59]$. The initial purpose of the $T M_{110}$ Mode Cavity was the generation of an RF separated Kaon beam in a fixed target experiment. Several superconducting versions of the cavity had been manufactured and tested with RF power, however, none were designed with the intention of installing into a beamline; further, neither did a beam-tube capable cryostat or a sufficient liquid helium supply exist. Since the emittance exchange field requirement was modest it was decided to construct the $T M_{110}$ Mode Cavity out of oxygen free, high conductivity (OFHC) copper.[60] A slightly higher $Q_{o}$ was required than what was achievable at room temperature with the OFHC copper. Referring back to Equations 5.7 and 5.8 we see that $Q_{o}$ is proportional to the square root of the copper's bulk conductivity. The conductivity of pure copper $\sigma_{c u}$, increases from $5.7 \times 10^{7}$ siemens/meter at room temperature to $9.6 \times 10^{7}$ siemens/meter at $l N_{2}$ temperature. A $Q_{o} 2.4$ times greater was achieved by simply incorporating a $l N_{2}$ cryogenic jacket into the design.

The integrated longitudinal acceleration of a particle traveling at the speed of light in this cavity is described by:

$$
V_{L}(r)=\frac{x \omega}{c} \sqrt{2\left(\frac{R}{Q}\right)^{\prime} P Q_{o} \frac{4 \beta}{(1+\beta)^{2}}}
$$




\begin{tabular}{|c|c|c|}
\hline Parameter & Value & Unit \\
\hline$f_{o}$ & 3.9 & $\mathrm{GHz}$ \\
\hline mode & $\pi, T M_{110}$ & \\
\hline body cell iris diameter & 30 & $\mathrm{~mm}$ \\
\hline end cell iris diameter & 36 & $\mathrm{~mm}$ \\
\hline equator diameter & 95.8 & $\mathrm{~mm}$ \\
\hline cell length & 38.4 & $\mathrm{~mm}$ \\
\hline$(R / Q)^{\prime}$ & 27.3 & $\Omega /$ cell \\
\hline Number of Cells & 5 & \\
\hline$Q_{o} @ 273 \mathrm{~K}$ & 14800 & \\
\hline$Q_{o} @ 80 \mathrm{~K}$ & 35600 & \\
\hline$Q_{L} @ 80 \mathrm{~K}$ & 20900 & \\
\hline$\Delta f_{f w h m} @ 80 \mathrm{~K}$ & 187 & $\mathrm{kHz}$ \\
\hline
\end{tabular}

Table 5.1: $T M_{110}$ Deflecting Mode Cavity Parameters

where $\left(\frac{R}{Q}\right)^{\prime}$ is the shunt impedance per unit length, $P$ is the incident RF power from the klystron, $Q_{o}$ is the natural $Q$ of the cavity, $\beta$ is the the coupling constant, $r$ is the distance off axis, $\omega$ is $2 \pi$ times the resonant frequency, and $c$ the speed of light in vacuo. Table 5.1 summarizes the relevant $T M_{110}$ Mode Cavity parameters.

Since a new, pulsed 80 kW, 3.9 GHz Varian Klystron (model VA-908K2) [64] was available, the copper $T M_{110}$ Mode Cavity was designed taking this Klystron's power constraint into consideration. A significant safety factor was built into the cavity structure in the event that the Klystron did not perform at the advertised $80 \mathrm{~kW}$, but rather as low as $50 \mathrm{~kW}$; estimates for losses in the circulator and waveguide were also taken into account. At 300 $\mathrm{K}$ the resonator would have required seven cells - however the mode spacing of a seven cell structure is too small causing undesired excitation of the next nearest mode, contaminating the $\pi$ mode. At $80 \mathrm{~K}$ the structure's $\mathrm{Q}$ increased by a factor of 2.4, reducing the overlap of the next nearest mode by both reducing the number of cells needed and by narrowing the resonances of each of the five $T M_{110}$ passband modes. In principle a three cell structure would have been sufficient but a five cell was built - the two extra cells providing the safety margin.

Selection of $f_{o}=3.9 \mathrm{GHz}$, being the $3^{r d}$ harmonic, minimized the effort to synchronize the $T M_{110}$ Mode Cavity with the A0 Photoinjector base operating frequency. In addition, since the longitudinal gradient scales with the frequency, a factor of three was gained by 
operating at the $3^{r d}$ harmonic. Since the maximum gradient for a given incident power occurs at unity coupling, $\beta \equiv Q_{o} / Q_{e}=1$, the coupling was set as close to unity as possible. The final $\beta$ achieved was 0.7 .

\section{$5.4 T M_{110}$ Mode Cavity Construction}

With the exception of one of the end half-cells, all the half cells were formed by stamping $2.2 \mathrm{~mm}$ thick OFHC copper disks at Advanced Energy Systems [48] using the same dies used in production of the Fermilab Niobium type C15a cavities. [63] The half cells were vacuum brazed at the iris, forming dumbbells. The dumbbells were electrically characterized and trimmed at the equator for the $\pi$ mode to resonate at $3.885 \mathrm{GHz}$ at $300 \mathrm{~K}$ in anticipation that the frequency would rise $12 \mathrm{MHz}$ due to thermal contraction at liquid nitrogen temperature as well as provide $3 \mathrm{MHz}$ for field flatness tuning. After trimming to the proper frequencies, each cell was found to be about 4 percent longer than the free space half wavelength at 3.9 $\mathrm{GHz}$, which corresponds to a 7 degree phase slip per cell for a relativistic particle. This did not pose a serious problem for EEX operation, as proper low level RF phase control and increased RF power compensated for it. The longer, suboptimum, cell length is a result of the different thermal contraction between copper $\left(\approx 17 \times 10^{6} /{ }^{\circ} \mathrm{C}\right)$ and niobium $\left(\approx 8 \times 10^{6} /{ }^{\circ} \mathrm{C}\right)$ and the necessity to chemically etch the niobium cavity but not the copper. Both effects led to a requirement to tune this cavity to lower frequency. A final vacuum braze of the dumbbells and end assemblies completed the structure.

The close proximity of the centerline of the coaxial input coupler $(20 \mathrm{~mm})$ to the iris of the end cell, required that the liquid nitrogen jacket, input coupler, and beam pipe all be machined into a single piece of copper. An RF pick-up probe was mounted on the beam pipe on the non-input coupler end. In order to protect their sealing surfaces, all of the stainless steel flanges were welded to previously brazed copper-to-stainless steel transitions only after the final braze. The thin walled, fully annealed, copper structure needed careful handling and constraints when pulling vacuum to prevent deformation and de-tuning. The liquid nitrogen jacket is a simple demountable stainless steel vessel which incorporates a bellows bridged by three pairs of lugs stiffened by threaded rods. The threaded rods provide both 


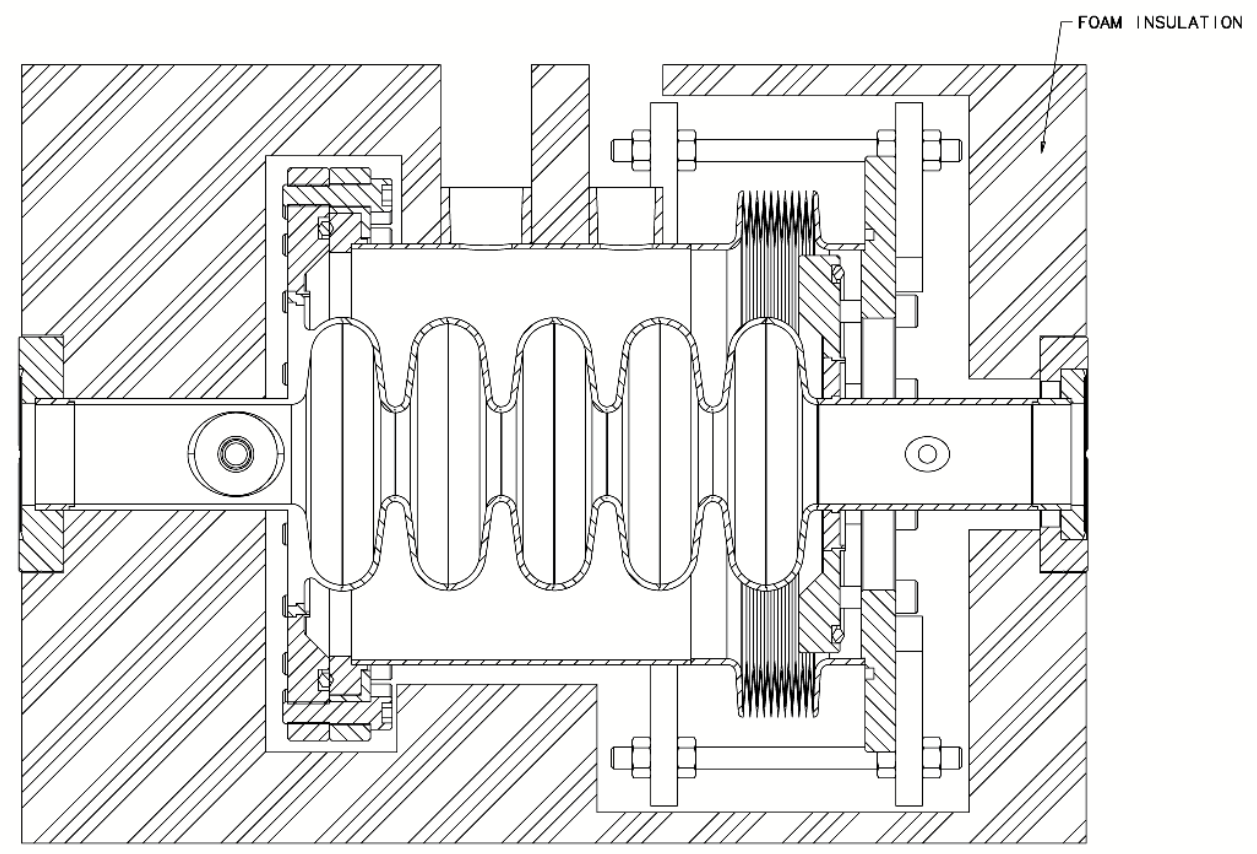

Figure 5.4: Cross sectional view of the $T M_{110}$ Mode Cavity with $l N_{2}$ jacket installed within the foam insulation

a method of fine tuning the cavity and the rigidity to constrain the cavity against vacuum forces. The liquid nitrogen jacket is sealed to the liquid nitrogen flanges with all metal aluminum diamond cross-section seals. Thermal insulation is provided by three layers of polyethylene foam cut to intimately contour the liquid nitrogen jacket, stiffening lugs, and plumbing fixtures. A polyethylene sheet is wrapped around the assembly, hermetically sealed with tape, and purged with dry nitrogen to prevent ice build-up.

\section{5 $T M_{110}$ Mode Cavity Input Power Coupler}

Since the joint FNAL/DESY $3.9 \mathrm{GHz} 3^{r d}$ Harmonic Cavity input coupler [50] has a power handling capacity of $80 \mathrm{~kW}$ pulsed, a spare was retrofitted for use with the EEX copper $T M_{110}$ Mode Cavity . The longitudinal location on the beam pipe and penetration into the beam pipe determines the coupling coefficient. The lower the cavity $Q_{o}$ the lower the $Q_{\text {ext }}$ must be, and hence the greater the coupling must be to realize a $\beta=1$. A coupling study was performed using HFSS to determine the placement of the 3rd Harmonic input 


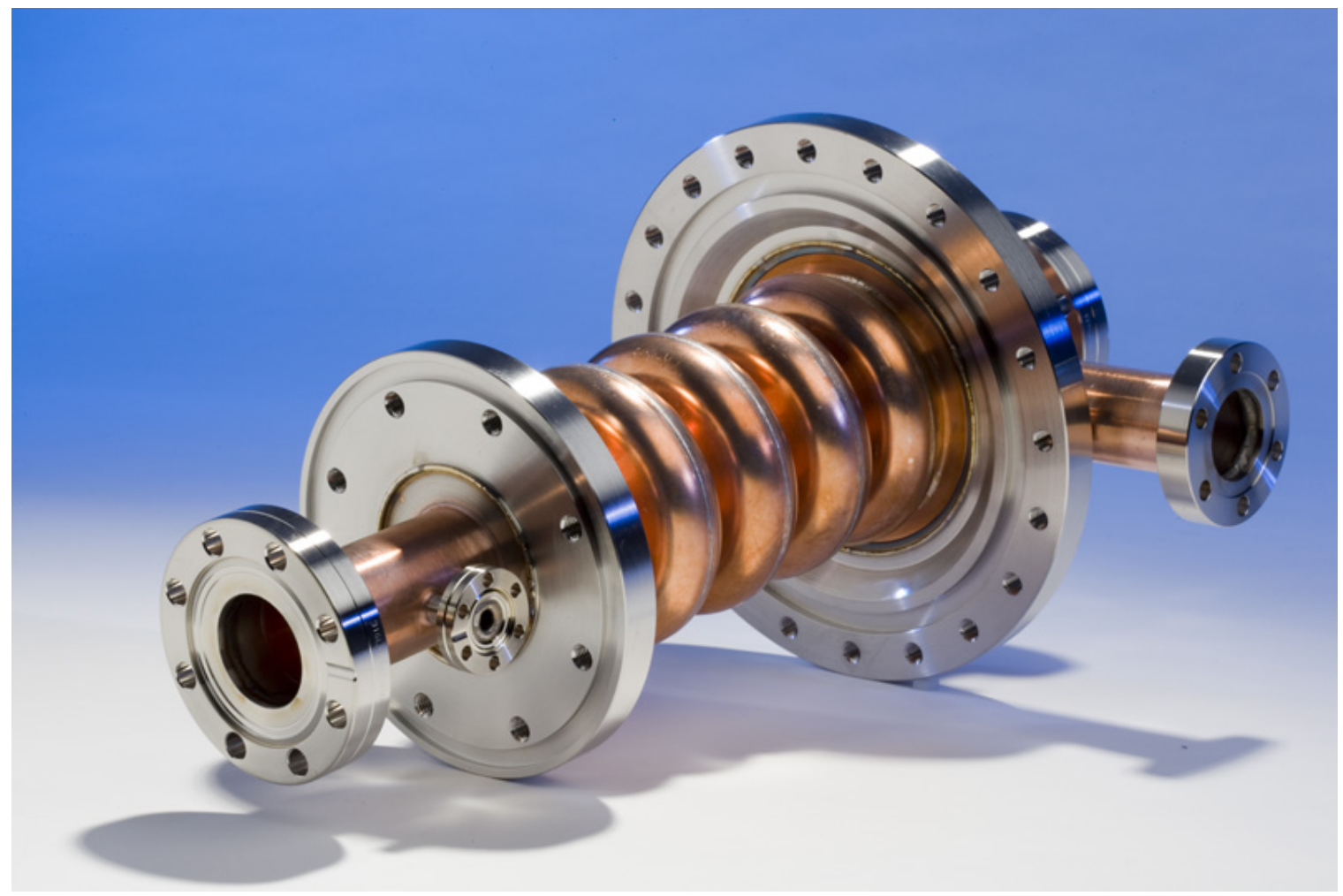

Figure 5.5: The fully brazed $T M_{110}$ Mode Cavity without its liquid nitrogen jacket

coupler for critical matching [54]. A five cell cavity with long beam pipes was modeled in the HFSS 3-D modeler. Two input couplers were incorporated to thoroughly simulate the input and pickup couplers through transmission measurements. To reduce the computation time, the modeled cavity structure was taken to be superconducting thus taking advantage of the ideal condition: $Q_{L}=Q_{\text {ext }}$. Since the copper structure $Q_{o}$ was anticipated to be 36,000 , the input coupler location was optimized for an transmission measurement reporting a $Q_{L}$ (thus $Q_{\text {ext }}$ ) of 36,000 . It was concluded that if the centerline of the input coupler resided $20 \mathrm{~mm}$ longitudinally away from the edge of the end cell, when the input coupler's tip was almost flush with the interior of the beam pipe wall, near unit coupling, $\beta \approx 1$, would occur. Slight empirical adjustment in the radial direction (penetration into the beam pipe) provided for fine tuning to $\beta=1$.

During initial low power RF testing the $T M_{110}$ Mode Cavity was outfitted with an adjustable model of the input coupler. The test input coupler had sufficient radial adjustment to go through unity coupling at both warm and cold temperatures. Unity coupling was empirically determined by noting the penetration that caused the minimum insertion loss 


\begin{tabular}{|c|c|c|}
\hline Radial Position of Tip [mm] & HFSS Simulated $Q_{\text {loaded }}$ & Calculated $\beta$ \\
\hline 14 & 12,300 & 2.92 \\
\hline 16 & 20,370 & 1.77 \\
\hline 18 & 34,190 & 1.05 \\
\hline 20 & 58,160 & 0.62 \\
\hline 22 & 100,600 & 0.36 \\
\hline 24 & 172,600 & 0.21 \\
\hline
\end{tabular}

Table 5.2: HFSS simulation to determine input coupler tip's radial penetration to reach unity coupling, $\beta=1$. The cavity $Q_{0}$ was taken to be $\infty$, thus $Q_{\text {loaded }}=Q_{\text {external }}$.

in an $S_{21}$ transmission measurement, where $S_{21}$ is the 2,1 element of the 'scattering matrix.' The scattering matrix is composed of four elements; $S_{11}$ and $S_{22}$ are measures of reflected signals originating from from ports 1 and 2 respectively, while $S_{21}$ and $S_{12}$ are measures of forward and reverse signal transmission through the device under test. For our discussion, $S_{21}$ simply is the ratio of the power transmitted through the cavity to the power incident on the cavity:

$$
S_{21}=\frac{P_{\text {transmitted }}}{P_{\text {incident }}}
$$

One can view $S_{21}$ as a measurement of insertion loss. The insertion loss is a minimum where $S_{21}$ is a maximum, thus the point of maximum power transfer. Figure 5.6 shows the data taken both warm and cold. $Q_{o}=14,800$ at $293 \mathrm{~K}$ and increased to 35,600 at $l N_{2}$ temperature. This increase is in agreement with the change in copper's conductivity between room temperature and $80 \mathrm{~K}$. The coupler's tip location for $\beta=1$ was found to be at $r=18$ $\mathrm{mm}$ cold, in excellent agreement with the calculation shown in Table 5.2. The location of $\beta=1$ cold corresponded to $\beta=0.4$ warm, also as expected from $\beta_{\text {warm }}=14,800 / 35,600$.

An OFHC center conductor extension was soldered to the spare high power DESY coupler to bring the tip to $r=18 \mathrm{~mm}$. During high power RF testing the coupling constant was again measured, using the steady state relationship of Equation 5.35.

$$
\beta=\frac{1 \pm \sqrt{\frac{P_{r e f}}{P_{f w d}}}}{1 \mp \sqrt{\frac{P_{r e f}}{P_{f w d}}}}
$$

with the upper sign for $\beta>1$ and the lower sign for $\beta<1$. Identifying from 5.7 that 


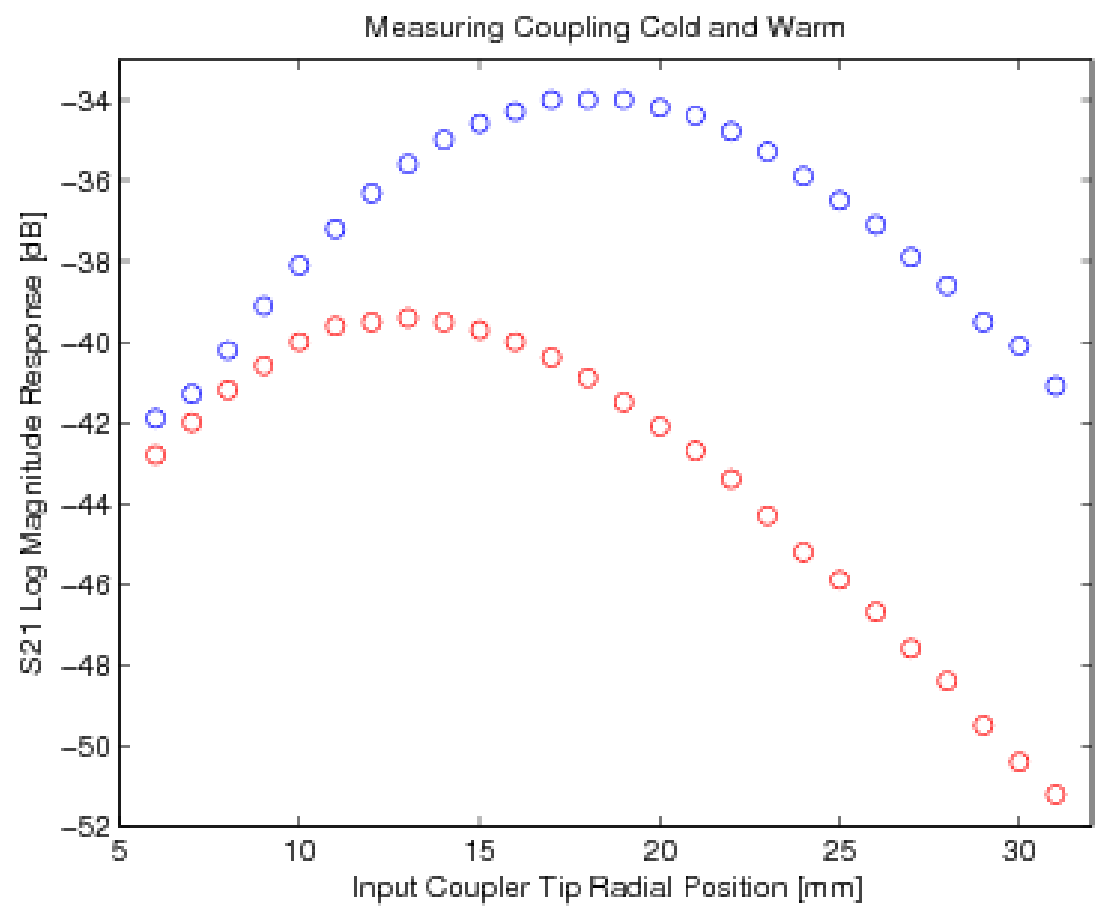

Figure 5.6: $S_{21}$ response against input coupler penetration at room temperature (red) and at $l N_{2}$ temperature (blue).

the cavity is slightly under-coupled [55], we calculated the coupling constant to be $\beta=$ $0.7 \pm 0.02$. The value of $\beta$ less than unity can be likely explained by several factors. First, the fixed length of the high power input coupler prevents fine adjustment of the couple radial penetration, and although careful specification of the coupler tip extension was executed, the variation in compression of the sealing gasket could not be anticipated. Further, since the field flatness of the structure cold is unmeasured, a slight reduction in field flatness is suspected.

\section{6 $T M_{110}$ Mode Cavity Field Flatness Tuning}

In order completely realize the maximum gradient to power efficiency, the $T M_{110}$ Mode Cavity structure was fine tuned such that each cell nearly equally contributed to the overall structure's gradient. The iterative perturbation technique of bead pulling was employed to reach an equal energy distribution in each cell, so called field flatness.

Slater's theorem relates the fractional change in a resonant cavity's frequency due to a 


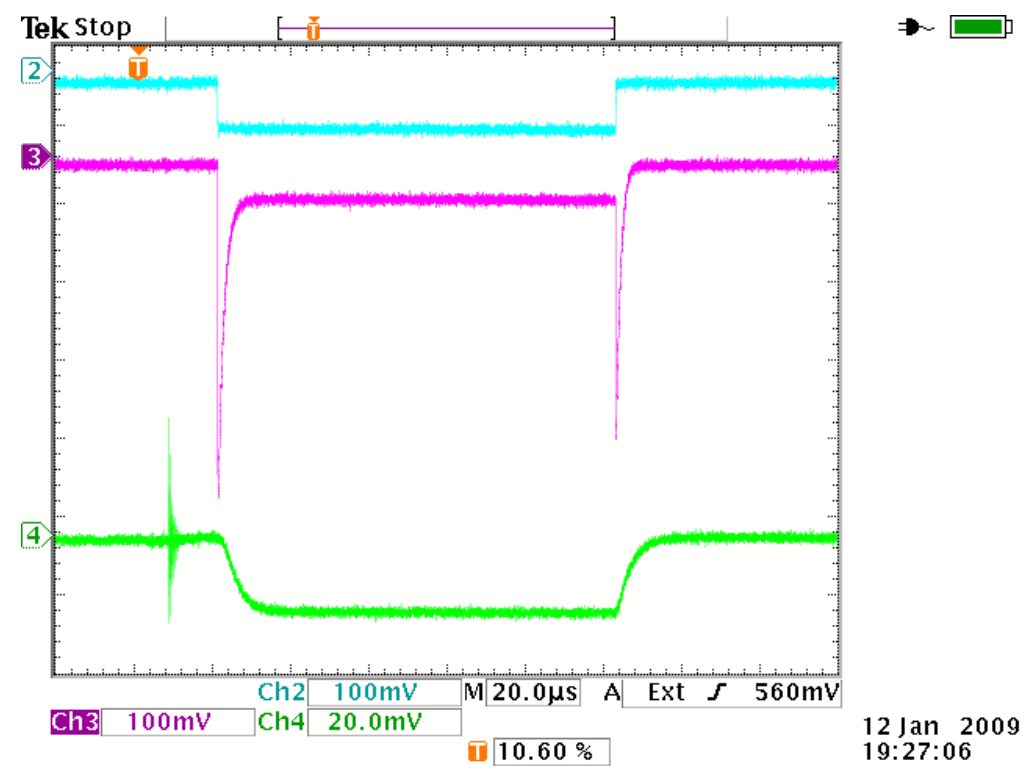

Figure 5.7: Oscilloscope traces of $T M_{110}$ Mode Cavity $P_{f w d}$ (blue), $P_{r e f}$ (red), $P_{\text {trans }}$ (green) diode signals during RF pulse.

small change in the resonators volume, $\Delta V$ by [61]:

$$
\frac{\Delta f_{o}}{f_{o}}=\frac{\int_{\Delta V}\left(\mu_{o} H^{2}-\epsilon_{o} E^{2}\right) d v}{\int_{V}\left(\mu_{o} H^{2}+\epsilon_{o} E^{2}\right) d v}=\frac{\Delta U_{\text {mag }}-\Delta U_{\text {elec }}}{U_{\text {total }}}
$$

where $U_{\text {total }}=\int_{V}\left(\mu_{o} H^{2}+\epsilon_{o} E^{2}\right) d V$ is the total of the stored time averaged magnetic and electric energy in the unperturbed resonator. $\Delta U_{m a g}=\frac{1}{4} \int_{\Delta V} \mu_{o} H^{2} d V$ and $\Delta U_{\text {elec }}=$ $\frac{1}{4} \int_{\Delta V} \epsilon_{o} E^{2} d V$ are the respective displaced, time averaged, stored magnetic and electric energies. In the case of a metallic bead of volume $\Delta V$ the fractional frequency change is found to be:

$$
\frac{\Delta f_{o}}{f_{o}}=-\frac{3 \Delta V}{4 U_{\text {total }}}\left(\epsilon_{o} E^{2}-\frac{\mu_{o} H^{2}}{2}\right)
$$

while in the case of a dielectric bead, where $\mu=1$ of volume $\Delta V$ :

$$
\frac{\Delta f_{o}}{f_{o}}=-\frac{3 \Delta V}{4 U_{\text {total }}} \frac{\epsilon-1}{\epsilon+2} \epsilon_{o} E^{2}
$$

A measurement of each cell's contribution is made by presenting an identical perturbation to each cell while monitoring the $T M_{110}$ Mode Cavity $\pi$ mode resonant frequency, as illustrated in Figure 5.8. Encoded in the resulting frequency shift is the direction and amount of tuning 
each cell required to bring the structure into field flatness at the resonant frequency. Since the goal was to obtain equal stored energy in each cell, determination of the absolute stored energy was not necessary, only each cell's relative frequency shift.

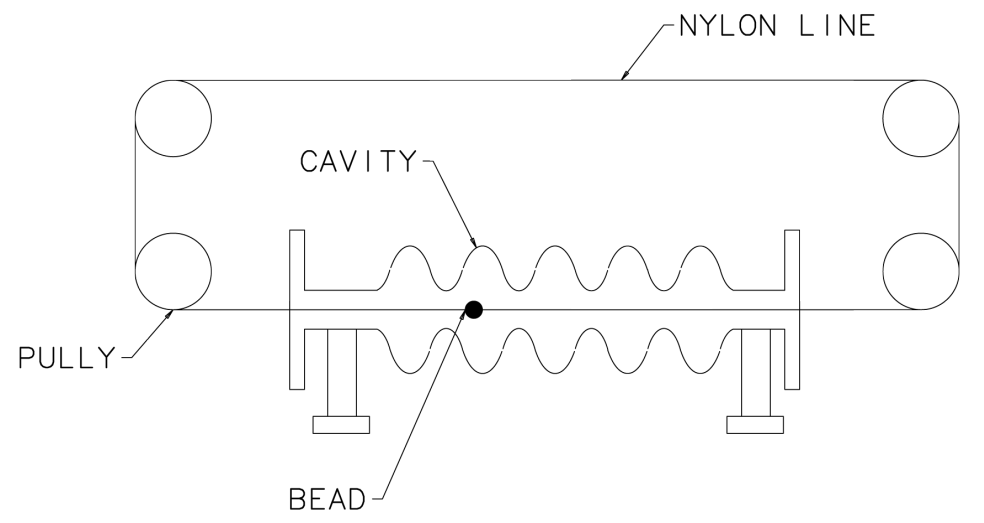

Figure 5.8: Conceptual diagram of the bead pull apparatus.

The apparatus used to perform the field flatness tuning was a resurrected Fermilab LINAC Bead Puller [49]. It consisted of a control computer interfaced with an HP8510 network analyzer and a stepper motor. Under computer control, the stepper motor assembly pulled a $1.3 \mathrm{~mm}$ diameter metallic bead along the longitudinal axis of the $T M_{110}$ Mode Cavity structure while the HP8510 recorded the relative phase shift near resonance of the $\pi$ mode. The HP8510 Network Analyzer inherently measures small changes in phase shift better than small changes in resonant frequency. The fractional change in frequency is determined by the relationship [62]:

$$
\frac{\Delta f}{f_{o}}=\frac{f-f_{o}}{f_{o}}=\frac{\tan \theta}{2 Q_{\text {loaded }}}
$$

The initial rms field flatness was about 20 percent. In principle, field flatness can be achieved by appropriately increasing and decreasing the resonant frequency of each cell. However, in our $T M_{110}$ Mode Cavity case, practical limitations only permitted tuning by squeezing the equator - resulting in an increase in frequency. Thus, the $3 \mathrm{MHz}$ overhead designed into the cavity's construction allowed for the field flatness condition to be approached from a frequency lower than the desired operating frequency. Again, since tuning was a one-way process, small tuning increments were made. After sixty some iterations field flatness was 
declared with less than a 2.6 percent rms spread in peak electric field, which was within the measurement system's resolution. After several thermal cycles between room temperature and $80 \mathrm{~K}$, the $T M_{110}$ Mode Cavity field flatness was remeasured. A slight reduction in flatness occurred, rising to $4.5 \%$ rms, but was still considered acceptable.

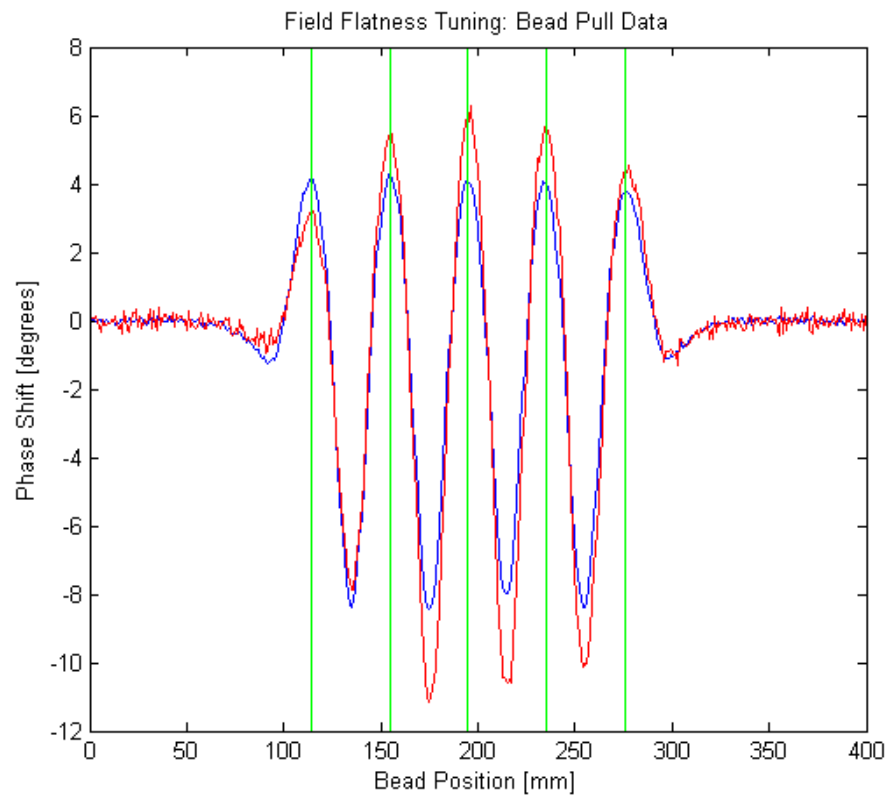

Figure 5.9: Overlay of initial (red) and final (blue) field flatness bead pulls. The vertical green lines indicate the equatorial plane of each cell.

\section{7 $T M_{110}$ Mode Cavity Polarization}

Unlike typical accelerating cavities that have azimuthally symmetric fields, the dipole mode of the $T M_{110}$ Mode Cavity structure necessarily has two polarizations which are azimuthally dependent. Their orientations need to be fixed and their frequencies separated. Typically, to orient the polarization a dimple is placed in a row on each cell, however, it was found that the input coupler perturbation of the structure was sufficient to force the orientation of the mode. The frequency separation of each polarization's $\pi$ mode is greater than $5 \mathrm{MHz}$. Generally the lower frequency polarization is used, as to avoid having neighboring modes at frequencies both higher and lower; however, the dominant polarization forced by the input coupler was the higher frequency mode, and the neighboring mode was considered to be far enough at $2.5 \mathrm{MHz}$ away. Again, the operating frequency could have 


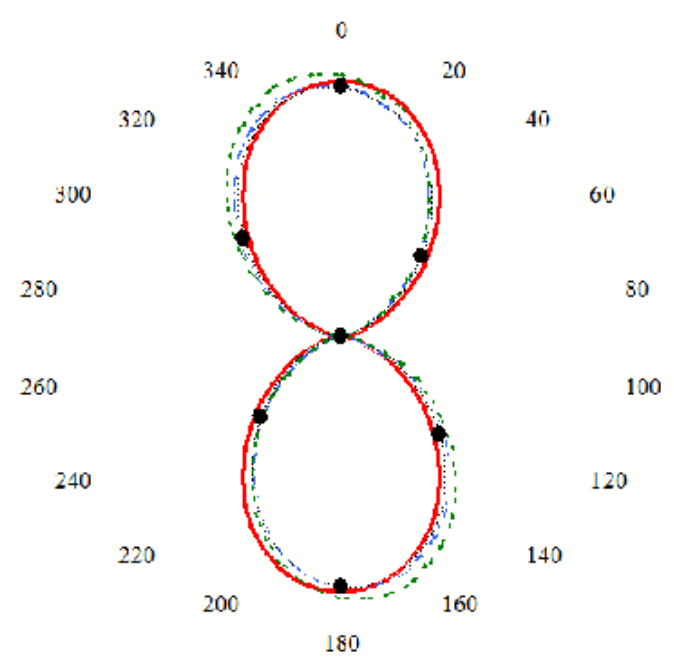

Figure 5.10: Final polarization plot. Red: ideal, black (shaded): fit Cell 2, blue (dashed): fit Cell 3, green (dots): fit Cell 4.

been lowered past that of the undesired polarization by dimpling, but at the risk of cracking a braze joint.

The cell to cell polarization was also measured through a bead pull technique. First the electrical center of the cavity was determined by a series of dielectric bead pulls in the plane of the peak $E_{z}$ field. Then the dielectric bead was pulled through the structure at eight locations $10 \mathrm{~mm}$ offset from the electrical center in a $45^{\circ}$ incremental circular pattern. The bead perturbations induced phase shifts that were measured and fit to the function $E^{2} \cos ^{2}(\theta)$. Figure 5.10 shows the resulting fit of the 8 points to cells 2,3 , and 4 . Since the off axis electric energy density does not go through a minimum in the equatorial plane of the end cells, the polarization could not be determined by this bead pull technique; their orientations were directly measured with small magnetic loop probes inserted on axis through their respective beam pipes. The end cell polarizations were found to be aligned with the body cells.

\section{$5.8 T M_{110}$ Mode Cavity Cryogenic Cooling}

Initial cavity cool down for low level testing and high power conditioning was extremely simple. A 160 liter pressure-building dewar was connected to the liquid nitrogen jacket by means of a vacuum insulated line. A pressure relief valve, set to 18 psia, was attached with 
an in-line tee prior to entrance of the $l N_{2}$ jacket to prevent an overpressure situation which could have permanently deformed the cavity. A second port on the $l N_{2}$ jacket exhausted the liquid into a vaporizer, and the resulting nitrogen gas was vented to the atmosphere. A flow meter was placed in the gaseous portion of the vent line to quantify the nitrogen flow. The cavity temperature was monitored by means of a type $\mathrm{K}$ thermocouple fixed to an exposed portion of the cavity copper near the input coupler. The dewar pressure and liquid exhaust valves were iteratively adjusted while monitoring the flow meter and temperature. The $l N_{2}$ flow was set just below threshold of the relief valve for initial cool down. The rate of cool down from room temperature to $80 \mathrm{~K}$ was limited to about 1 hour. Once the cavity temperature indicated that the jacket was full of liquid, the flow was throttled back to read 350 Standard Cubic Feet per Hour (SCFH) on the exhaust flow meter. At steady state, the system typically ran for 6 hours without need for adjustment. Pressure fluctuations in the system caused the cavity's resonant frequency to shift $\pm 40 \mathrm{kHz}$ with a time constant measured in seconds; ultimately pressure regulation improvements were incorporated into the permanent installation.

After the testing phase was complete, the $T M_{110}$ Mode Cavity was installed in the EEX beamline. The permanent $l N_{2}$ supply system was designed to continuously maintain the five cell $T M_{110}$ Mode Cavity at $80 \mathrm{~K}$ while preserving the Photoinjector cave's oxygen deficiency hazard $(\mathrm{ODH})$ classification at 0 [65]. The source of $l N_{2}$ came from an existing 1500 gallon, 250 PSI pressure building dewar located outside of the A0 building. The $l N_{2}$ was piped to a feed box which houses a cryogenic electric valve that in turn provides regulation of $l N_{2}$ flow. Following the electric valve, a flexible vacuum jacketed line transported the $l N_{2}$ to the five cell $T M_{110}$ Mode Cavity . The exhaust line was of similar construction to the test installation discussed above - except that aperture requirements were increased to maintain the ODH classification. A pressure transducer was connected into the $l N_{2}$ supply line immediately before the five cell $T M_{110}$ Mode Cavity $l N_{2}$ jacket; the pressure signal regulated the electric valve. In addition to the type-K thermocouple, two platinum resistors were mounted to the exterior of the $l N_{2}$ jacket for temperature monitoring. The pressure and temperatures were permanently data-logged.

As expected, during the first cold tests, thermal contraction increased the frequency 


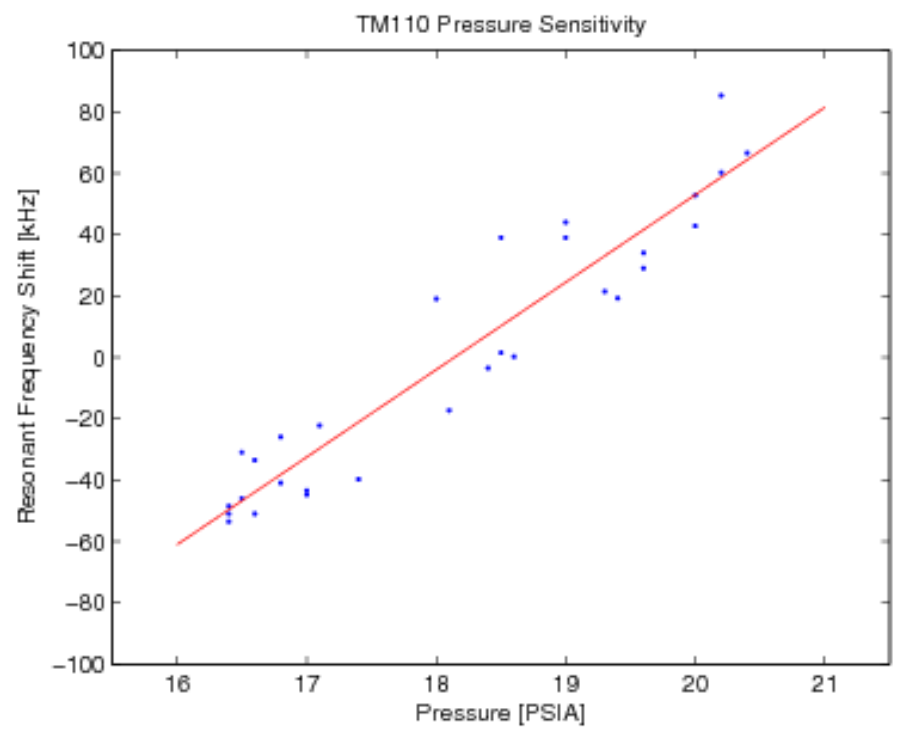

Figure 5.11: $T M_{110} \pi$ mode sensitivity to $l N_{2}$ bath pressure change

approximately $11.8 \mathrm{MHz}$ to $3.89930 \mathrm{GHz}$. Adjustment of the three threaded rods brought the structure into resonance. For occasional facility maintenance, the $T M_{110}$ Mode Cavity had to be thermally cycled. It was found, that after cool down equilibrium ( 24 hours ) each cycle shifted the $\pi$ mode resonant frequency up to $100 \mathrm{kHz}$.

Pressure sensitivity was measured to be about $29 \mathrm{kHz} / \mathrm{PSI}$ and is shown in Figure 5.11. After the cavity was cold for 24 hours, the fine tuning was complete, the final tuning goal was $3.9 \mathrm{GHz} \pm 30 \mathrm{kHz}$, however, up to $\pm 50 \mathrm{kHz}$ was considered acceptable.

\section{9 $T M_{110}$ Mode Cavity First Beam}

Although, at the time of this writing, the original design of this $3.9 \mathrm{GHz}$ deflecting $T M_{110}$ Mode Cavity is over a decade old, and several superconducting versions have been built and tested with RF power, this emittance exchange experiment's copper version has been the first to experience beam. On September 18, 2007, a $15 \mathrm{MeV}$ beam was propagated through the EEX line, and the $T M_{110}$ Mode Cavity was energized to a modest gradient with $8.5 \mathrm{~kW}$. While recording the outgoing beam's horizontal position at BPM26 the $T M_{110}$ Mode Cavity RF phase was scanned 360 degrees in 5 degree steps. The resulting deflection is plotted in Figure 5.12, a maximum deflection of $\pm 7 \mathrm{~mm} 520 \mathrm{~mm}$ down stream of the 
center of the cavity was noted, resulting in an effective $V_{\perp}$ kick of $200 \mathrm{kV}$.

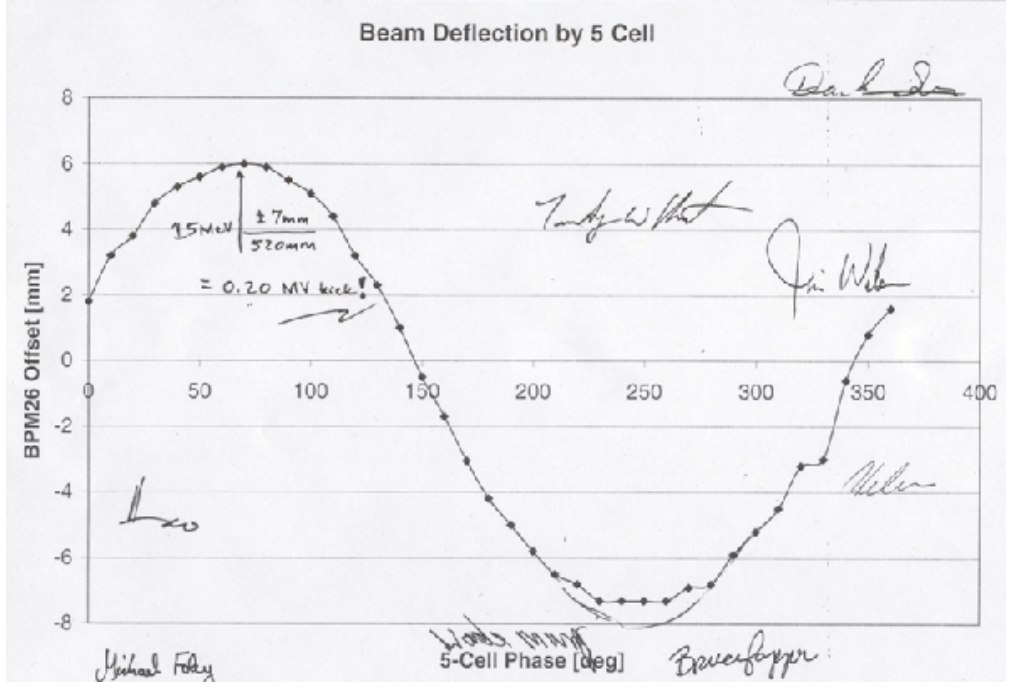

Figure 5.12: Signed plot of first beam deflection using a FNAL built $T M_{110}$ Mode Cavity structure September 18, 2007.

It was quickly observed that the transverse gradient was not as strong as anticipated for the amount of power being delivered to the cavity: $8.5 \mathrm{~kW}$ at unity coupling with a $Q_{o}=35,600$ a transverse kick of $290 \mathrm{kV}$ was expected. This was attributed to the less than unity coupling, $\beta=0.7$, the sub-optimal length, and the suspected reduction of field flatness under cold conditions. The two contingency cells of the five cell structure and increased RF drive power brought the cavity to the needed gradient. Careful tuning of the cavity, the LLRF controls, and with sufficient RF power to the cavity ( $55 \mathrm{~kW}$ ), the gradient needed for the emittance exchange condition was achieved. The most dramatic display of the cavity reaching the required gradient will be shown in Figure 6.13.

The $3.9 \mathrm{GHz}$ operational support systems, HLRF and LLRF, have been described previously in section 4.2 .

\subsection{Beam Based Electrical Center}

A beam-based alignment similar to the method developed at DESY [66] was used to determine the electrical center of the $T M_{110}$ Mode Cavity with respect to beam position monitors immediately before and after the cavity, BPM25 and BPM26 respectively. While still at room temperature, a a $15 \mathrm{MeV}$ electron bunch train of twenty 1-nC bunches was 


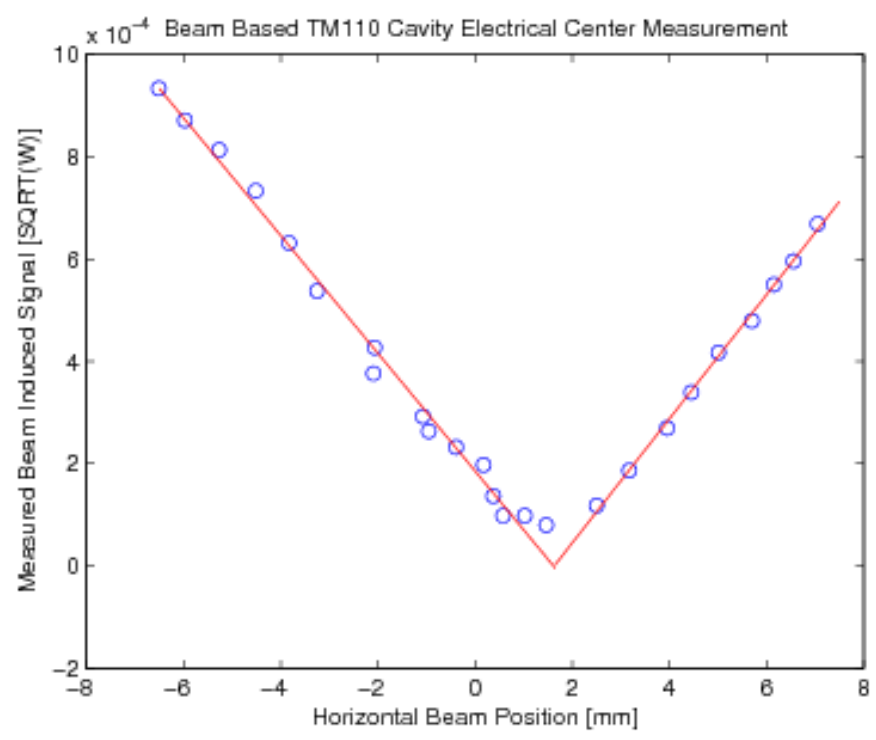

Figure 5.13: Beam based determination of $T M_{110}$ Mode Cavity electrical center.

propagated through the EEX beamline and the $T M_{110}$ Mode Cavity . A spectrum analyzer, in zero-span mode tuned to the warm $\pi$-mode frequency $(\approx 3.885 \mathrm{GHz})$, was connected to the cavity's RF pickup port. Using two horizontal correctors upstream to generate horizontal offsets, the electron beam was horizontally stepped across the aperture of the $T M_{110}$ Mode Cavity in $1 \mathrm{~mm}$ increments while the peak amplitude of the induced signal on the pickup port was recorded. Figure 5.13 shows the resulting plot with linear fits indicating the electrical center of the $T M_{110}$ Mode Cavity corresponds to a combined horizontal BPM25, BPM26 reading of $1.6 \mathrm{~mm}$. This procedure was again attempted after the $T M_{110}$ Mode Cavity was cooled to $l N_{2}$ temperatures, however, the cool down brought the $f_{o}=3.9$ GHz. The gun's unwieldy dark current, having $1.3 \mathrm{GHz}$ structure, greatly excited the $\pi$ mode. The dark current signal swamping the beam induced signal, thereby making the cold measurement too difficult to pursue. Ultimately, a measurement of the change in the beam's central momentum between the $T M_{110}$ Mode Cavity on and off states indicated that the cold electrical center of the cavity did not significantly differ from the warm state.

The horizontal tilt of the cavity with respect to BPM25 and BPM26 could be similarly determined, again by using the upstream correctors to generate a composite angle and offset while recording the induced signal. This was deemed unnecessary and hence not performed. 


\section{Chapter 6 Measurement of EEX Transport Matrix}

\subsection{EEX Transport Matrix Measurement Introduction}

After the installation and initial commissioning of the emittance exchange apparatus the first priority was a verification of the emittance exchange transport matrix. The method of difference orbits was employed to measure the emittance exchange beamline matrix. The reader is referred to Figure 4.11 as an atlas to the EEX beamline and the relative placement of components used in the acquisition of the EEX matrix. Additionally, Table A.2 of Appendix A contains the longitudinal position of these elements. Diagnostic and beam control limitations required that a fully measured matrix include a $2.1 \mathrm{~m}$ drift prior to the first EEX dipole magnet and a $0.2 \mathrm{~m}$ drift following the fourth EEX dipole. Thus, the beginning of the EEX beam line was defined to be at the center of trim magnet HTBX03, located at $s=3706 \mathrm{~mm}$ from the cathode, and the end of the EEX beamline was defined as being at BPM28, $s=9782 \mathrm{~mm}$ from the cathode. At least partial transport matrix elements can be measured at every BPM location, which may be a useful future endeavor, however, the initial goal was to obtain a full measure of the collective $6 \times 6$ emittance exchange transport matrix.

To aid the reader throughout this chapter, I describe matrix elements by both their indices as well by partial derivatives. This will serve to emphasize the element's 'function;' for example, the $R_{12}$ element is a measure of $\partial x_{o u t} / \partial x_{i n}^{\prime}$, or the change in output horizontal offset with a change in the input horizontal angle. 


\subsection{Measurement Scheme}

The difference orbit matrix measurement procedure established a reference (nominal) 14.3 $\mathrm{MeV}, 5^{\circ}$ off-crest, $1 \mathrm{nC}$ beam orbit through the EEX beamline and measure both the 6-D input and output vectors. Then one of the 6-D input vector components was varied: $\Delta x_{i n}, \Delta x_{i n}^{\prime}, \Delta y_{i n}, \Delta y_{i n}^{\prime}, \Delta z_{i n}$, or $\Delta \delta_{i n} ;$ while all of the changes in components of the 6D output vector were measured, $\Delta x_{\text {out }}, \Delta x_{\text {out }}^{\prime}, \ldots$, and $\Delta \delta_{\text {out }}$. As an example, by only changing the input horizontal angle, $\Delta x^{\prime}$, and by measuring all of the resulting changes in the output parameters, the entire $2^{\text {nd }}$ column of the transport matrix can be inferred.

$$
\left(\begin{array}{c}
\Delta x \\
\Delta x^{\prime} \\
\Delta y \\
\Delta y^{\prime} \\
\Delta z \\
\Delta \delta
\end{array}\right)_{\text {out }}=\left(\begin{array}{cccccc}
R_{11} & R_{12} & R_{13} & R_{14} & R_{15} & R_{16} \\
R_{21} & R_{12} & R_{13} & R_{14} & R_{15} & R_{16} \\
R_{31} & R_{12} & R_{13} & R_{14} & R_{15} & R_{16} \\
R_{41} & R_{12} & R_{13} & R_{14} & R_{15} & R_{16} \\
R_{51} & R_{12} & R_{13} & R_{14} & R_{15} & R_{16} \\
R_{61} & R_{12} & R_{13} & R_{14} & R_{15} & R_{16}
\end{array}\right)\left(\begin{array}{c}
0 \\
\Delta x^{\prime} \\
0 \\
0 \\
0 \\
0
\end{array}\right)_{\text {in }}
$$

To demonstrate the 'turn-on' evolution of the EEX matrix, this procedure was repeated with the $T M_{110}$ Mode Cavity off, at the full strength for the complete EEX condition, as well as at three intermediate strengths. The BPM readings gave $\Delta x, \Delta x^{\prime}, \Delta y$, and $\Delta y^{\prime}$, while the streak camera provided the $\Delta z$ information, and the vertical bending spectrometer in conjunction with the subsequent vertical BPM position reading provided the output $\Delta \delta$ data. The selection of $14.3 \mathrm{MeV}$ was based on the anticipated operating momentum of the initial EEX program; the bunch charge of $1 \mathrm{nC}$ was to ensure sufficient intensity to trigger the BPM system. Finally, the 9-Cell cavity was operated 5 degrees off crest where minimum bunch momentum spread occurs, yet still sufficiently near crest to minimize phase induced momentum jitter. 


\subsection{Input Parameter Changes}

The beam input parameter changes consisted of offsets in transverse position, transverse angle, beam time of arrival (longitudinal offset), and central momentum.

Both input vertical and horizontal position offsets, $\Delta x_{i n}$ and $\Delta y_{i n}$, were generated by a pair of horizontal and vertical corrector dipoles, H/VTAX9C followed by H/VTBX03. Both transverse input angles, $\Delta x_{i n}^{\prime}$ and $\Delta y_{i n}^{\prime}$, were controlled by correctors H\&VTBX03 only.

To avoid the complexities of adjusting the Photoinjector's electron bunch arrival time, the phase of the $T M_{110}$ Mode Cavity was simply adjusted to produce differing bunch timeof-arrival $\Delta t$, and correspondingly, $\Delta z_{i n}$. This is justified since except for the $T M_{110}$ Mode Cavity, all of the EEX beamline components are static, thus a phase advance of $1^{\circ}$ of the $T M_{110}$ Mode Cavity is equivalent to $0.7 \mathrm{ps}$ earlier arrival time, or a $0.2 \mathrm{~mm}$ longitudinal offset.

Finally, the change in input momentum, $\Delta \delta$ was generated by adjusting the gradient of the superconducting 9-cell cavity.

\subsection{Data Collection \& Reduction}

\subsubsection{Measuring Matrix Elements using BPMs}

It this section we describe the BPM based measurement techniques used in obtaining matrix elements. As discussed in Chapter 4, the BPMs measure the transverse centroid of the electron bunch, and thus were used for measuring the beam's transverse offsets. Each subsection describes the measurement of a representative matrix element in a given column. The columns are introduced in order of increasing measurement complexity.

All of the $6 \times 6$ matrix elements, except for the fifth row, could be obtained through BPM measurements. The fifth row's elements are measures of output longitudinal position (or electron bunch time of arrival with respect to the reference particle) dependence on the input parameters and thus were determined with the streak camera that are addressed in Section 6.4.2. During BPM data acquisition, for thoroughness and universality, each triggered BPM beam event was recorded. Each event record contained information from 


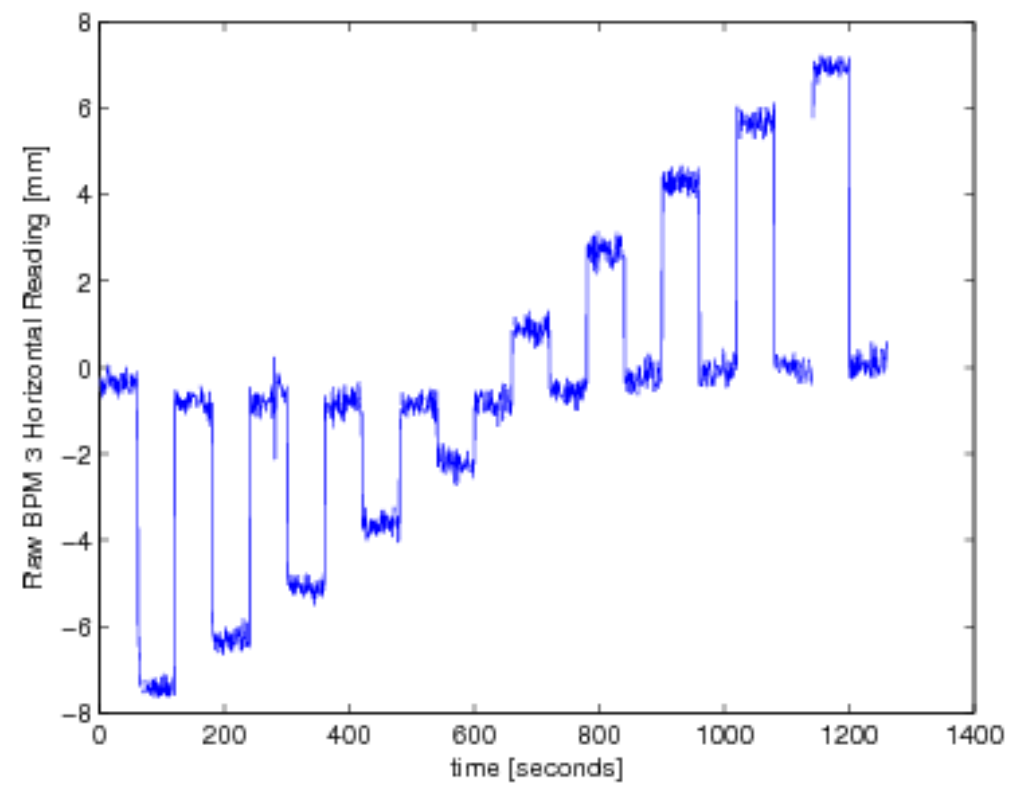

Figure 6.1: Strip chart of raw BPM 3 horizontal signal during $\Delta x^{\prime}$ input scan, BPM2 and BPM28 signals are similar.

the ten BPM locations: the calculated transverse beam position in $\mathrm{mm}$, the raw signal strength from all four BPM buttons, and each BPM's ADC attenuation setting. This yields a pulse by pulse transverse snapshot of the entire beam line.

\section{Measurement of the $2^{\text {nd }}$ Column}

The measurement of the $R_{12} \equiv \partial x_{\text {out }} / \partial x_{\text {in }}^{\prime}$ element is representative of all of the BPM difference orbit measurements, and will serve to describe the method employed. We start with a measurement in the $2^{\text {nd }}$ column, $\left(\partial x_{i n}^{\prime}\right)$, since it is one of the more 'pure' column measurements able to be made in the sense that only one beamline element is changed.

The elements $R_{12}\left(x_{\text {out }}=R_{12} x_{\text {in }}^{\prime}\right)$, can in principle, be measured by noting the ratio of the horizontal change at BPM28 against a change in input angle obtained from the telescope formed by BPM2 and BPM3 separated by distance $l_{3-2}$ :

$$
R_{12}=\frac{\partial x_{\text {out }}}{\partial x_{\text {in }}^{\prime}}=\frac{\Delta B P M 28_{x}}{\left(\triangle B P M 3_{x}-\Delta B P M 2_{x}\right) / l_{3-2}}
$$

To establish linearity, this was repeated for several points in $\left(x_{i n}^{\prime}, x_{o u t}\right)$ coordinate space.

The data collection began after establishing the nominal central orbit. To establish a 
baseline, 60 BPM beam events (1 minute of RF pulses) were recorded. A control script stepped the horizontal dipole corrector, HTBX03, through a series of currents imparting a sequence of input offset angles. For statistics, 60 beam events were recorded for each input angle offset, after which the dipole corrector magnet was returned to the nominal orbit setting where another 60 events were taken. The return to the nominal orbit in between each offset value monitored for potential drifts during the angle scan. Figure 6.1 is a plot of the BPM3 horizontal reading throughout the duration of the angle scan, BPM2 and BPM28 plots are similar. The range of angles was limited to keep the beam in the linear response region of all of the BPMs, $\pm 10 \mathrm{~mm}( \pm 15 \mathrm{~mm}$ for BPM8 and BPM30). Of the gathered data, the pertinent data set for each $\left(\Delta x_{i n}^{\prime}, \Delta x_{\text {out }}\right)$ measurement can be represented by Table 6.1. There are three column pairs: the offset measurement followed by the background for BPM2, BPM3, and BPM28 for 60 beam events.

\begin{tabular}{|c|c||c|c||c|c|}
$X_{2}^{o n}[1]$ & $X_{2}^{o f f}[1]$ & $X_{3}^{o n}[1]$ & $X_{3}^{o f f}[1]$ & $X_{28}^{o n}[1]$ & $X_{28}^{o f f}[1]$ \\
$X_{2}^{o n}[2]$ & $X_{2}^{o f f}[2]$ & $X_{3}^{o n}[2]$ & $X_{3}^{o f f}[2]$ & $X_{28}^{o n}[2]$ & $X_{28}^{o f f}[2]$ \\
$X_{2}^{o n}[3]$ & $X_{2}^{o f f}[3]$ & $X_{3}^{o n}[3]$ & $X_{3}^{o f f}[3]$ & $X_{28}^{o n}[3]$ & $X_{28}^{o f f}[3]$ \\
$\vdots$ & $\vdots$ & $\vdots$ & $\vdots$ & $\vdots$ & $\vdots$ \\
$X_{2}^{o n}[i]$ & $X_{2}^{o f f}[i]$ & $X_{3}^{o n}[i]$ & $X_{3}^{o f f}[i]$ & $X_{28}^{o n}[i]$ & $X_{60}^{o f f}[i]$ \\
$\vdots$ & $\vdots$ & $\vdots$ & $\vdots$ & $\vdots$ & $\vdots$ \\
$X_{2}^{o n}[60]$ & $X_{2}^{o f f}[60]$ & $X_{3}^{o n}[60]$ & $X_{3}^{o f f}[60]$ & $X_{28}^{o n}[60]$ & $X_{60}^{o f f}[60]$
\end{tabular}

Table 6.1: Representation of $R_{12}$ data set

In Table 6.1 $X_{B P M}$ indicates a horizontal reading at indexed subscript 'BPM' and the index $[i]$ is the beam event. The superscript on implies the input offset is active, while off refers to the background state.

As can be seen in Figure 6.1, there is variation during the measurement, so the first step in the post-processing analysis of the horizontal angle offset data was to subtract the mean value of the $60 \mathrm{BPM}$ background readings for each BPM event. The background in this measurement of $R_{12}$ was the mean value of the nominal 60 events subsequent to the corrector change. Since this is a delta measurement, using the preceding nominal 60 events would have been equally legitimate. However, due to hysteresis in the iron based corrector element, the prior and post nominal input angles were different, therefore extrapolating from their means would be erroneous. 


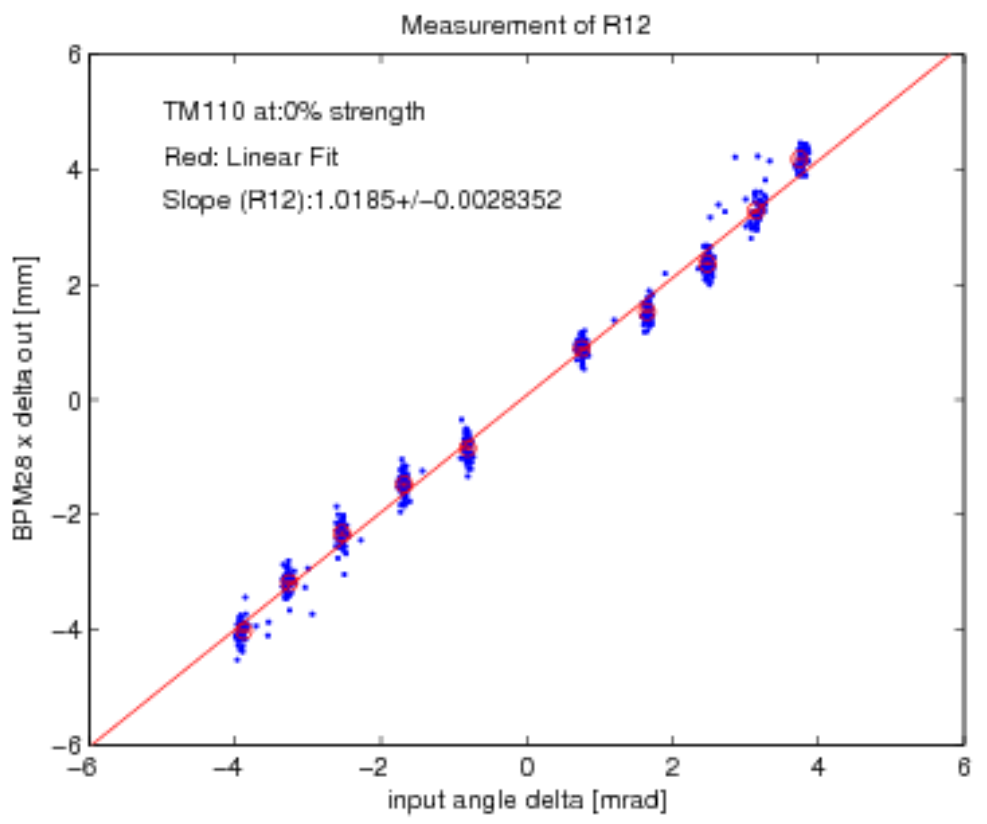

Figure 6.2: $\left.\left(x_{i n}^{\prime}, x_{o u t}\right)\right|_{x_{i, j}^{\prime}}$ points, $\left.\left(x_{i n}^{\prime}, x_{o u t}\right)\right|_{x_{j}^{\prime}}$ means and standard deviation of the mean, and a weighted least squares fit to the mean values.

Equation 6.2 illustrates the algorithm used to calculate $\left(x_{i n}^{\prime}, x_{\text {out }}\right)$ for each offset-background pair. To reduce the BPM common mode noise, the summation calculates $R_{12}$ for each beam event simultaneously, but subtracts the mean of the backgrounds:

$$
\left(x_{\text {in }}^{\prime}, x_{\text {out }}\right)=\left(\sum_{i=1}^{N} \frac{\left(X_{28}^{o n}[i]-\frac{\sum_{B=1}^{k} X_{28}^{\text {off }}[B]}{k}\right)}{\left\{\left(X_{3}^{o n}[i]-\frac{\sum_{B=1}^{k} X_{3}^{o f f}[B]}{k}\right)-\left(X_{2}^{o n}[i]-\frac{\sum_{B=1}^{k} X_{2}^{o f f}[B]}{k}\right)\right\} / l_{3-2}}\right) / N
$$

At each $\left.\left(x_{i n}^{\prime}, x_{\text {out }}\right)\right|_{x_{j}^{\prime}}$ an rms was calculated for a weighted linear fit, where subscript $j$ indicates each offset value. Figure 6.2 shows the the the individual calculated $\left.\left(x_{i n}^{\prime}, x_{o u t}\right)\right|_{x_{i, j}^{\prime}}$ points (where subscript $i$ indicates each beam event), their mean values, $\left.\left(x_{i n}^{\prime}, x_{o u t}\right)\right|_{x_{j}^{\prime}}$ and the standard deviation of the mean. A weighted linear least squares fit to the $\left.\left(x_{i n}^{\prime}, x_{o u t}\right)\right|_{x_{j}^{\prime}}$ data is also plotted in Figure 6.2,its slope provides the measure of $R_{12}$ with its uncertainty: $1.02 \pm 0.003 \mathrm{~mm} / \mathrm{mrad}$.

This scan was repeated for the five $T M_{110}$ Mode Cavity strengths $k_{0}=0 \%, 25 \%, 50 \%$, $75 \%$, and $100 \%$. Figure 6.3 plots the evolution of $R_{12}$ against the $T M_{110}$ Mode Cavity 


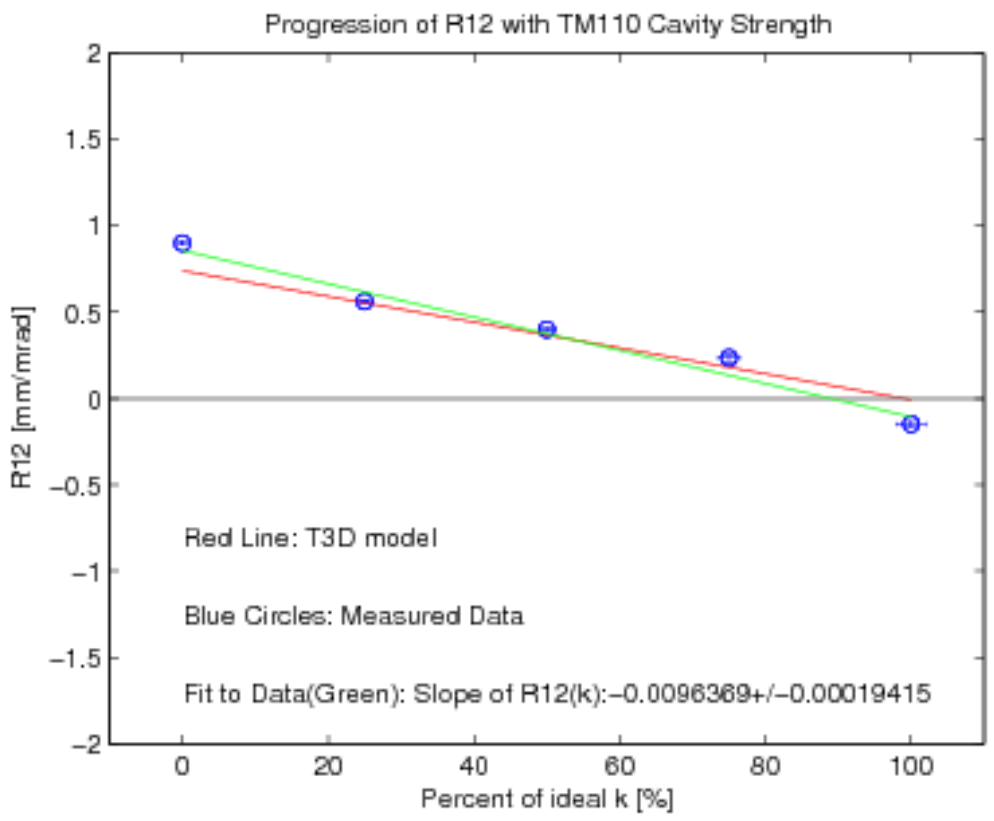

Figure 6.3: Evolution of the $R_{12}$ element as the $T M_{110}$ Mode Cavity strength is ramped. The vertical error bars indicate the standard deviation of the mean.

strength. A least squares linear fit was made to the cavity data and is compared to our T3D-MatLab matrix calculations.

The recorded BPM delta data provides sufficient information to deduce the entire $2^{\text {nd }}$ column of the EEX transport matrix, with the exception of the element $R_{52}, \partial z_{\text {out }} / \partial x_{\text {in }}^{\prime}$ which required measurement by the streak camera. Thus this analysis procedure was repeated to measure the $R_{22} \equiv \partial x_{\text {out }}^{\prime} / \partial x_{i n}^{\prime}, R_{32} \equiv \partial y_{\text {out }} / \partial x_{i n}^{\prime}, R_{42} \equiv \partial y_{\text {out }}^{\prime} / \partial x_{i n}^{\prime}$, and the $R_{62} \equiv \partial \delta_{\text {out }} / \partial x_{\text {in }}^{\prime}$ elements. The output angles, $\Delta x_{\text {out }}^{\prime}$ and $\Delta y_{\text {out }}^{\prime}$, in determining $R_{22}$ and $R_{42}$, were obtained through the telescope formed by BPM28 and BPM29, similar to the BPM2-BPM3 input telescope. In measuring $R_{62}$ the output fractional momentum offset change, $\Delta \delta_{\text {out }}$ was obtained through the vertical position measured at BPM30, located in the dispersive section after EEX spectrometer magnet, where the vertical dispersion at BPM30 was measured to be $865 \pm 10 \mathrm{~mm}$.

\section{$R_{12}$ Trajectories}

During the above $R_{12}$ measurement, complete $\Delta x$ and $\Delta y$ data was recorded at all ten BPM locations. Figures 6.4 through 6.8 show plots of both the horizontal (left) and 
vertical (right) difference BPM values. Vertical lines are drawn to indicate the longitudinal position of key components. In ascending order they are Dipole 1, Dipole 2, the $T M_{110}$ Mode Cavity , Dipole 3, Dipole 4, and the EEX spectrometer. The first longitudinal data point is BPM2, and follows through to BPM30's located after the spectrometer. These plots effectively demonstrate the beam's trajectories for the different values of $\Delta x_{i n}^{\prime}$. For comparison, the T3D-MatLab expected values are plotted as solid lines. The dashed lines are included to provide a connect-the-dot convenience, and should not be considered as the actual trajectory. Figure 6.4 starts with the $T M_{110}$ Mode Cavity off, $k=0 \%$, the subsequent plots are of increasing cavity strength, with Figure 6.8 displaying the $T M_{110}$ Mode Cavity at full strength. This sequence illustrates the conversion of an input transverse parameter to a longitudinal output parameter. As the $T M_{110}$ Mode Cavity is ramped, the output angles, $\Delta x_{\text {out }}^{\prime}$, are reduced while the output momentum offset, $\Delta \delta_{\text {out }}$ increases. This is seen by the change in the vertical trajectory at BPM30 which resides in the vertical dispersive line following the spectrometer. Most notably, at the $T M_{110}$ Mode Cavity strength of $k=k_{o}$, the output angles are almost completely suppressed, while large momentum changes are observed. This shows that input transverse parameters map to output longitudinal parameters, indicating features of emittance exchange.

These trajectory measurements were used to determine the effective bending radius used in T3D, as described in Section 4.3.7. With a bending radius of $\rho_{o}=900 \mathrm{~mm}$ there is good agreement with the T3D-MatLab calculations. In the $k=0.75 k_{o}$ data set, the last two trajectories of the positive offsets have poor agreement with the T3D-MatLab model, and depart from the characteristic behavior, of the previous five trajectories. The trajectories first depart from the expected values at BPM24, located between Dipoles 1 and 2, the first BPM located in a dispersive section $(D \approx 165 \mathrm{~mm})$. The offset departs even more at BPM25, located in a region of greater dispersion $(D \approx 330 \mathrm{~mm})$, just before the entrance into the $T M_{110}$ cavity. These excursions were most likely due to either a sudden change in either the laser, gun, or 9-Cell phase, creating a sudden change (drop) in momentum, effecting the 'purity' of the input $\Delta x^{\prime}$. At this point $k=0.75 k_{o}$, it is difficult to use the EEX spectrometer information as the function of the EEX line compensates for input momentum differences. However the resulting horizontal output offset and angle are telling. As a result, 
close monitoring of the laser, RF Gun, and 9-Cell systems was maintained for subsequent measurements 

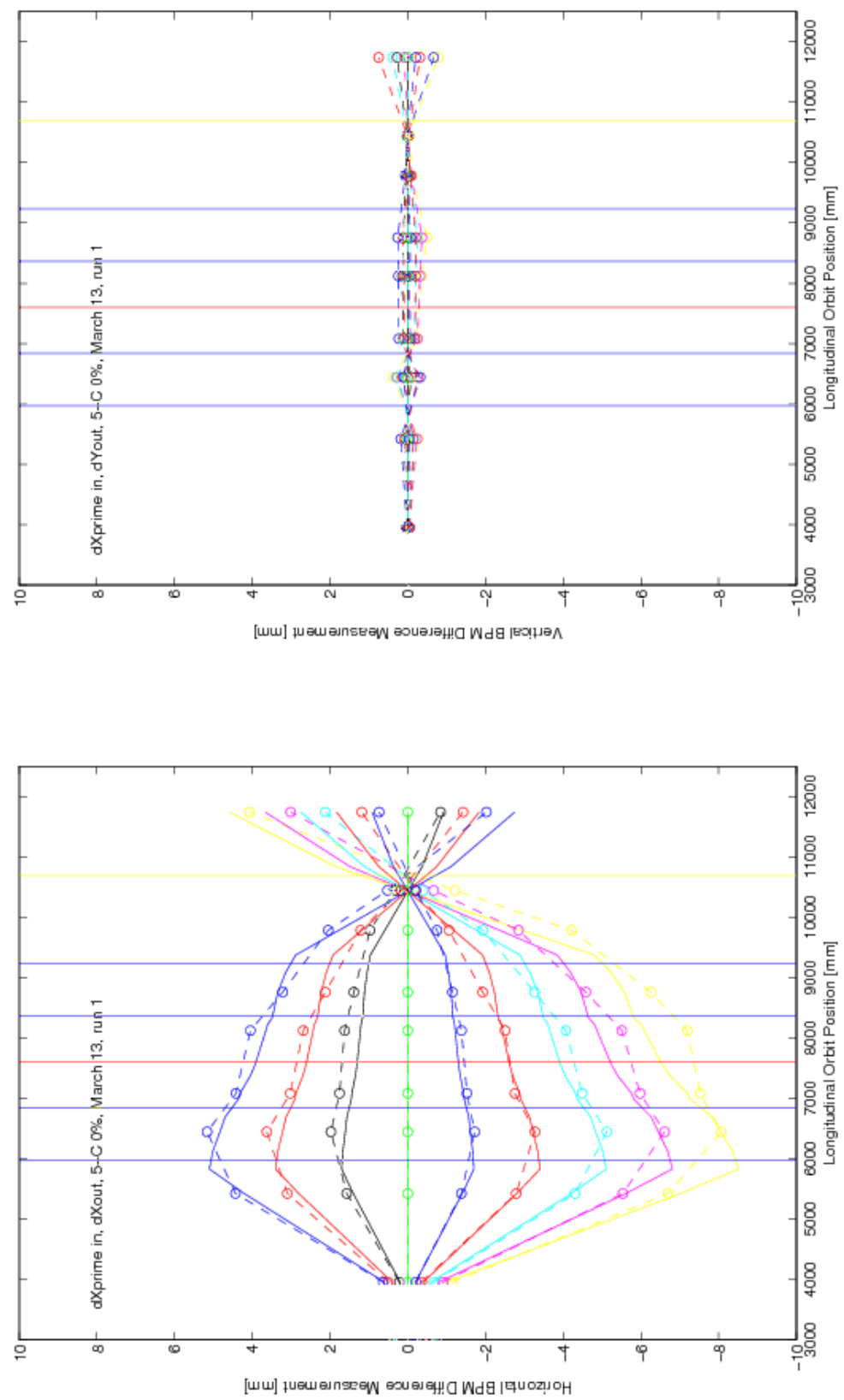

Figure 6.4: Horizontal \& vertical BPM delta measurements for differing input $\Delta x^{\prime}$ plotted against longitudinal beamline position for $k=0 \% k_{o}$, forming horizontal trajectories. Solid lines are calculated T3D-Matlab values for comparison. Dashed lines only 'connect the data dots' and are not to be taken as the trajectories.(Note image is rotated $90^{\circ}$ for viewing) 

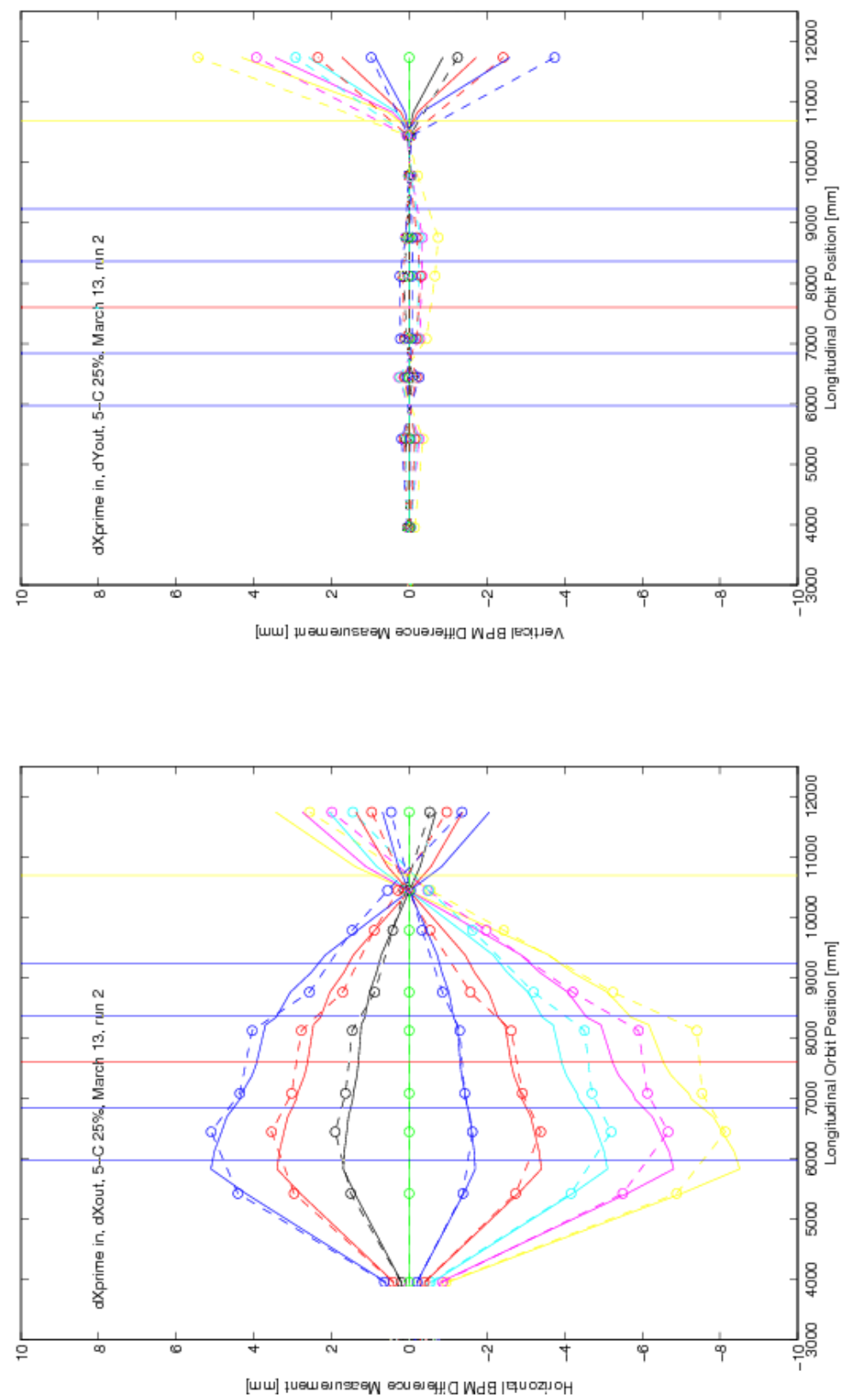

Figure 6.5: Horizontal \& vertical BPM delta measurements for differing input $\Delta x^{\prime}$ plotted against longitudinal beamline position for $k=25 \% k_{o}$, forming horizontal trajectories. Solid lines are calculated T3D-Matlab values for comparison. Dashed lines only 'connect the data dots' and are not to be taken as the trajectories.(Note image is rotated $90^{\circ}$ for viewing) 

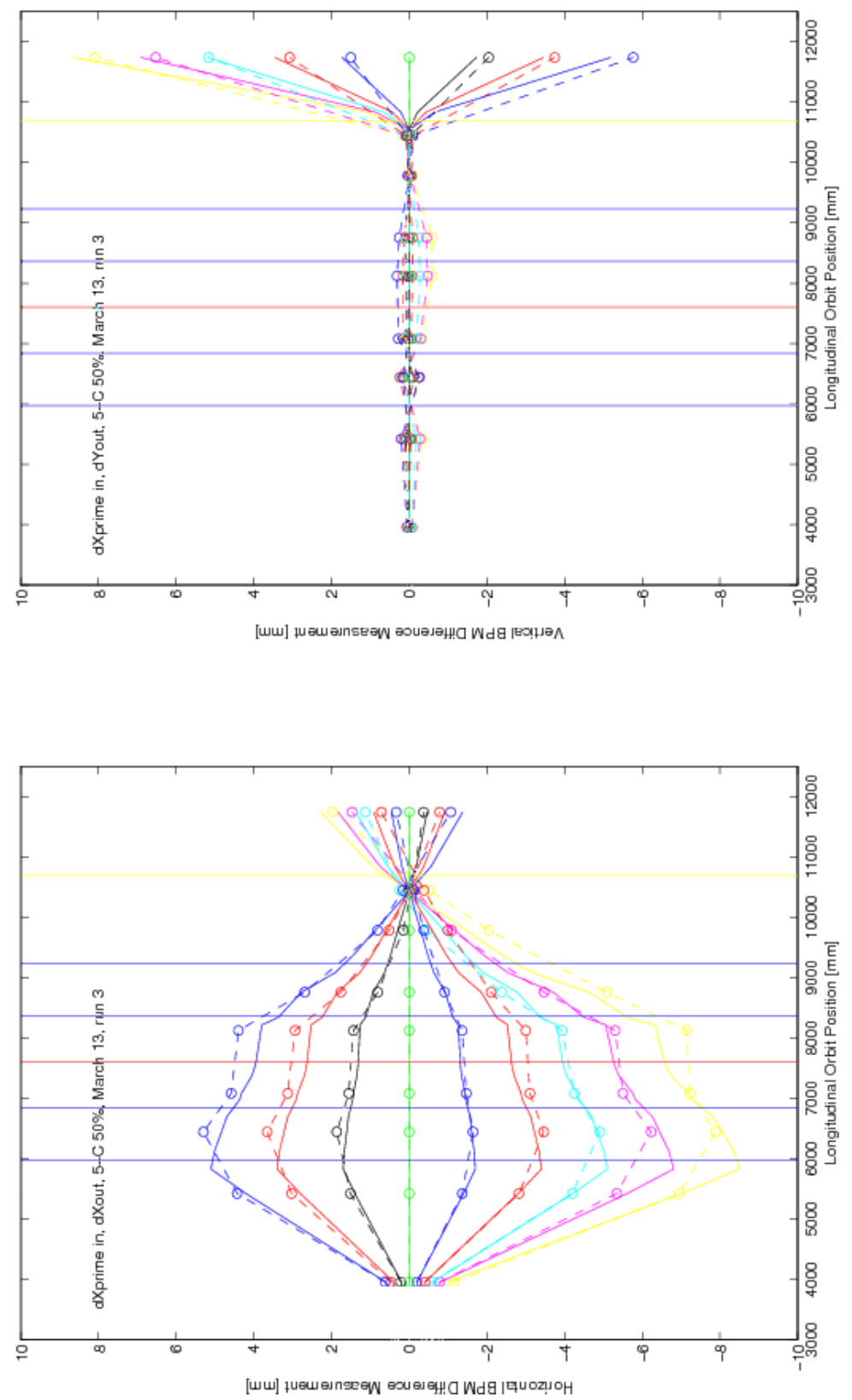

Figure 6.6: Horizontal \& vertical BPM delta measurements for differing input $\Delta x^{\prime}$ plotted against longitudinal beamline position for $k=50 \% k_{o}$, forming horizontal trajectories. Solid lines are calculated T3D-Matlab values for comparison. Dashed lines only 'connect the data dots' and are not to be taken as the trajectories.(Note image is rotated $90^{\circ}$ for viewing) 

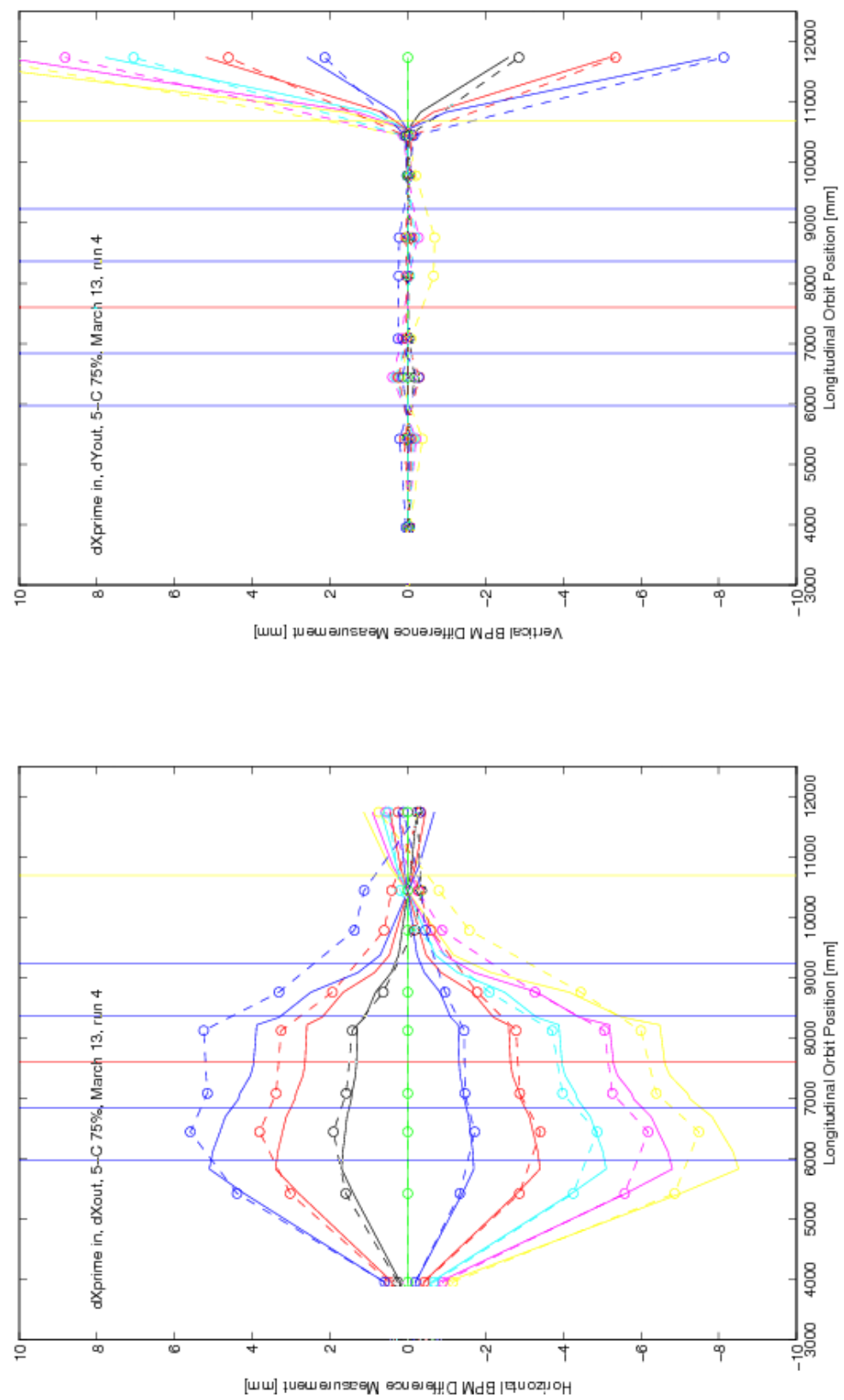

Figure 6.7: Horizontal \& vertical BPM delta measurements for differing input $\Delta x^{\prime}$ plotted against longitudinal beamline position for $k=75 \% k_{o}$, forming horizontal trajectories. Solid lines are calculated T3D-Matlab values for comparison. Dashed lines only 'connect the data dots' and are not to be taken as the trajectories.(Note image is rotated $90^{\circ}$ for viewing) 

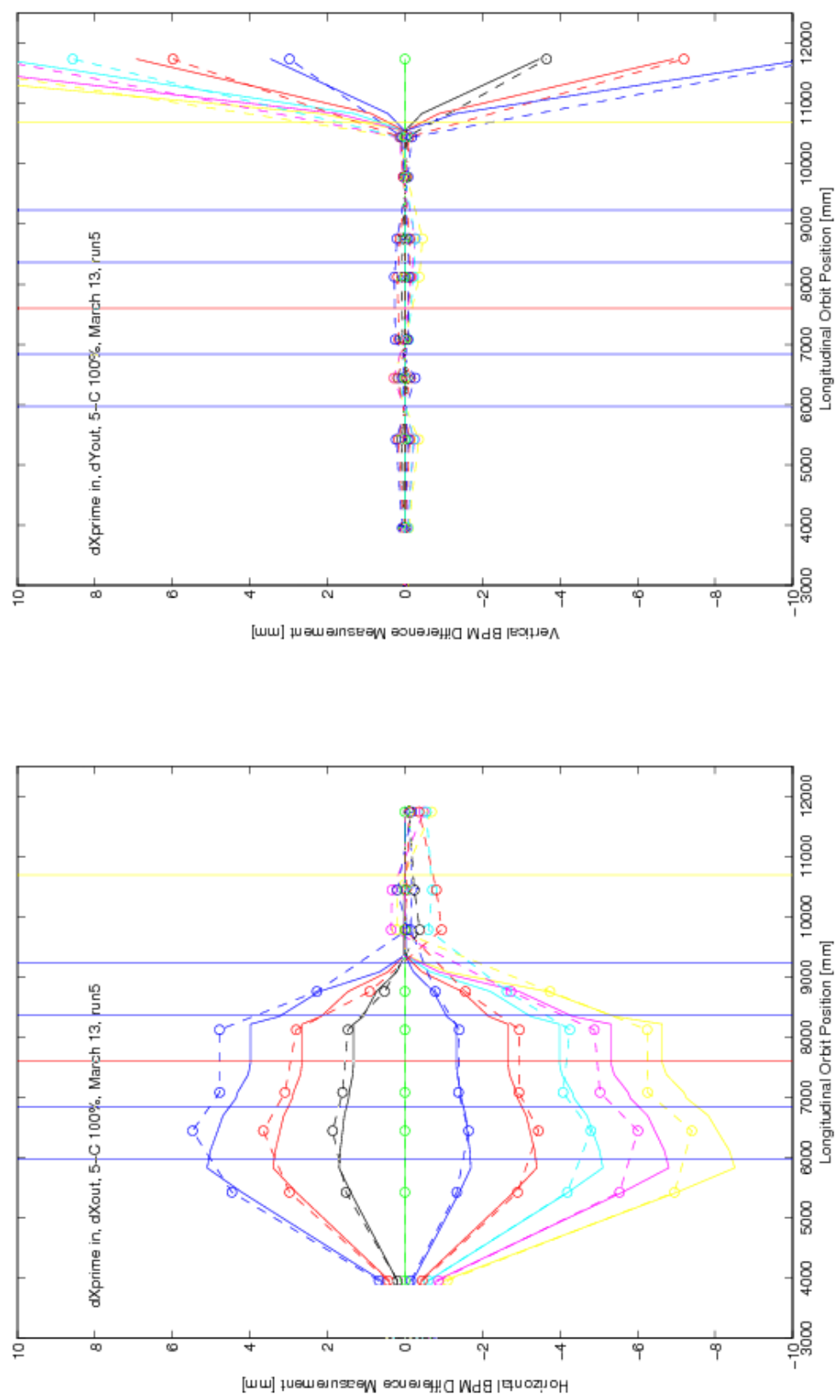

Figure 6.8: Horizontal \& vertical BPM delta measurements for differing input $\Delta x^{\prime}$ plotted against longitudinal beamline position for $k=100 \% k_{o}$, forming horizontal trajectories. Solid lines are calculated T3D-Matlab values for comparison. Dashed lines only 'connect the data dots' and are not to be taken as the trajectories.(Note image is rotated $90^{\circ}$ for viewing) 


\section{$\mathbf{R}_{12}$ Reproducibility}

$R_{12}$ was remeasured several months later. The results of the two measurements are shown in Figure 6.9. A variation larger than the statistical error bars was observed, indicating a systematic change. Since a direct observation of the emittance exchange was deemed more pertinent, as well as good agreement at $k=k_{o}$, this was not further explored. The variation between measurements is used to assign uncertainties in the final matrix values.

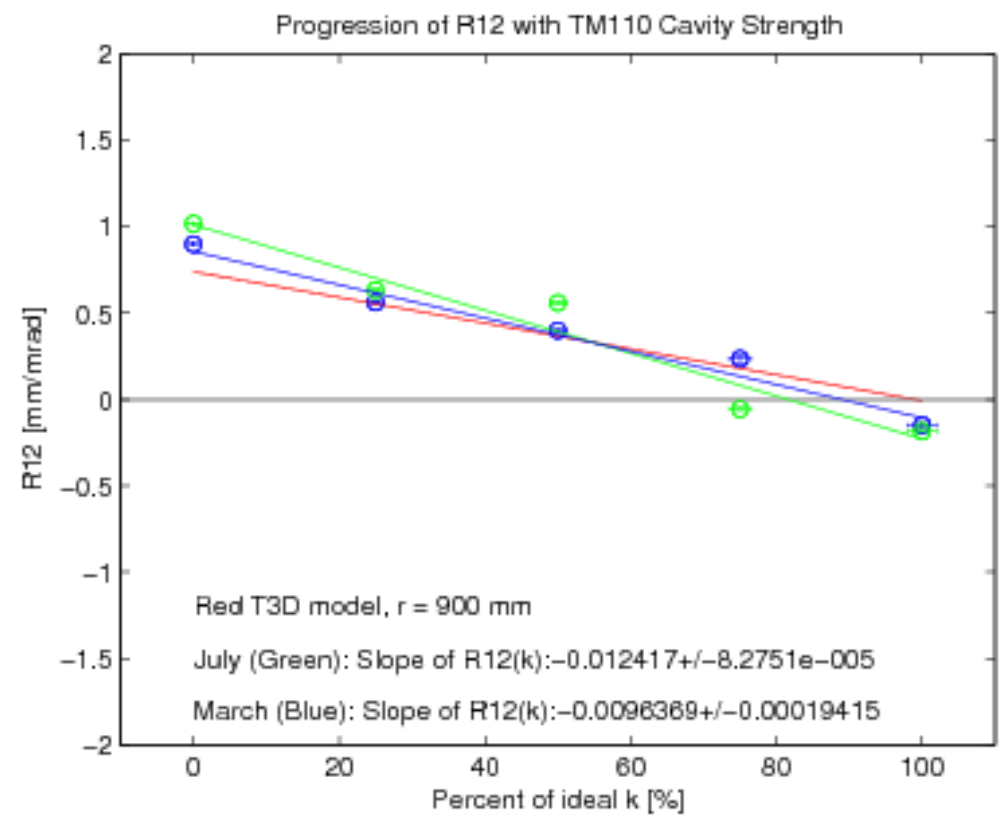

Figure 6.9: Comparison of two $R_{12}$ measurements. The vertical error bars indicate the standard deviation of the mean.

\section{Measuring Matrix Column 1}

In the above section, we considered the measurements of the second column elements to be 'pure' in the sense that using a single corrector dipole we could obtain an input angle without incurring an unwanted transverse offset as well. However, in the case of measuring the first column, due to the manual nature of setting the two longitudinally spaced correctors (H\&VTAX9C and H\&VTBX3) to reproducibly generate a horizontal input offset, $\Delta x_{i n}$, this made it difficult to avoid some input contamination angle offset, denoted as $\theta_{i n}$.

Because of the manual nature and difficulty in reproducibility of returning to a specific 
nominal beam trajectory, a slightly different measurement approach was taken in measuring the first column. Before data collection began, effort was expended to ensure that BPM2 and BPM3 on average read within $5 \%$ of each other. Data collection started with $\Delta x_{i n}=$ $-6 \mathrm{~mm}$ stepping to $+6 \mathrm{~mm}$ in $2 \mathrm{~mm}$ increments, taking the standard 60 beam events at each incremental step. This was repeated for the usual five $T M_{110}$ Mode Cavity strengths. The distributions of input angles for each cavity strength are displayed in Figure 6.10. Then BPM28 $\Delta x_{\text {out }}$ vs. BPM2 $\Delta x_{\text {in }}$ was plotted in $\left(x_{i n}, x_{o u t}\right)$ coordinate space. A single data set near $x_{i n}=0$ was used as the background value otherwise the analysis was identical to the previously discussed $R_{12}$ data. The slope from a weighted linear least squares Fit to the means of $\left.\left(x_{i n}, x_{o u t}\right)\right|_{x_{j}}$ data is a measure of the $R_{11}$ matrix element. The evolution of $R_{11}$ with the $T M_{110}$ Mode Cavity strength isplotted in Figure 6.12.
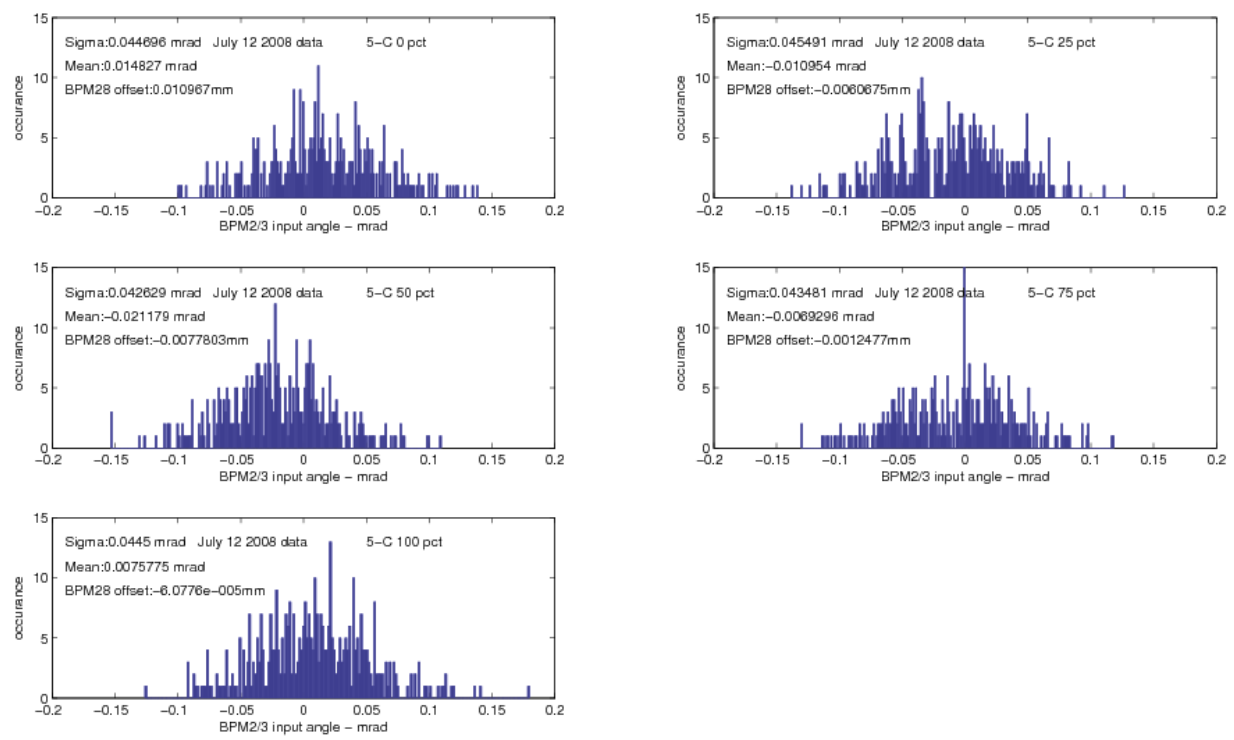

Figure 6.10: Distribution of input angles during $R_{11}$ measurement 

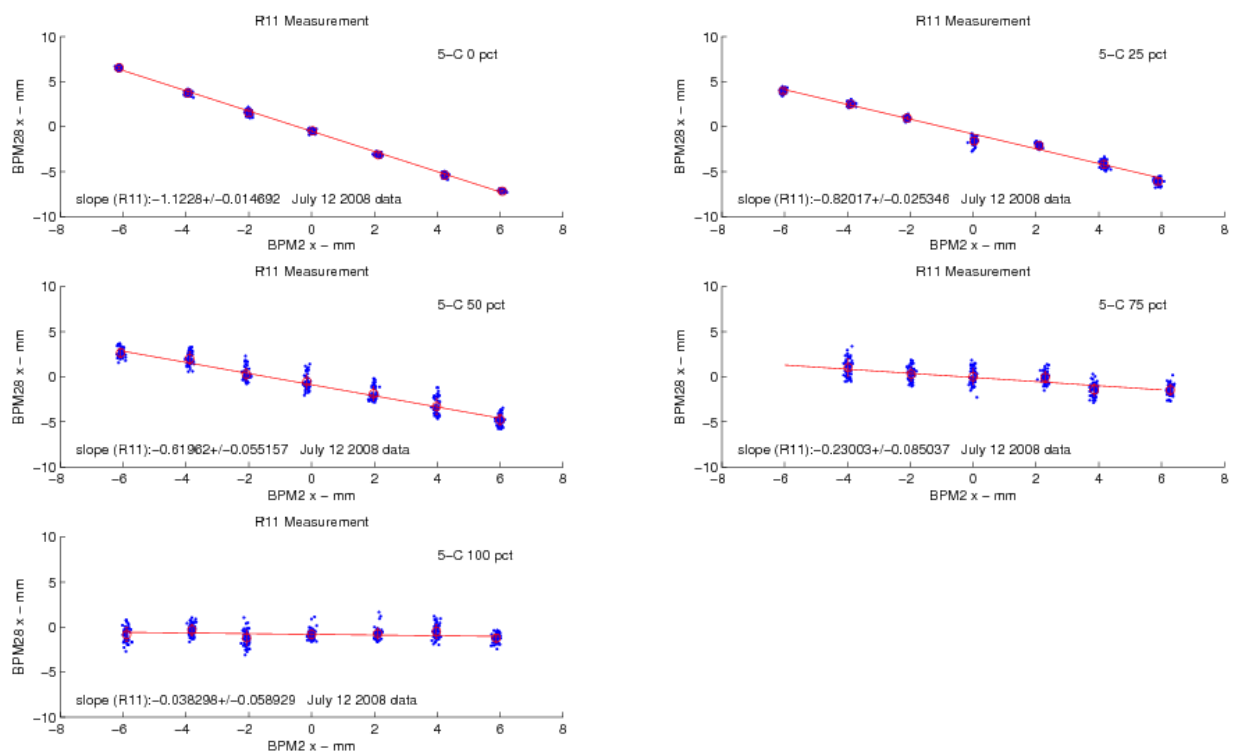

Figure 6.11: Measuring $R_{11}$ : horizontal BPM28 reading plotted against horizontal BPM3 reading

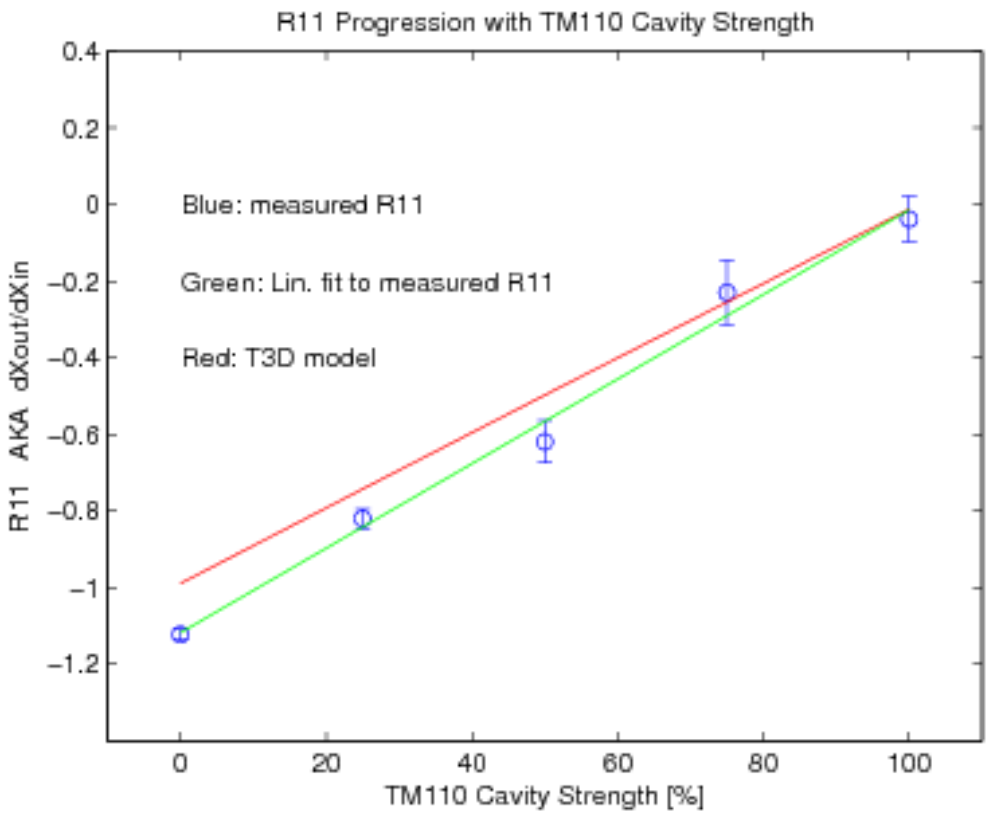

Figure 6.12: Evolution of the $R_{11}$ element as the $T M_{110}$ Mode Cavity strength is ramped. A linear fit (green) to the evolution data is compared to the T3D model (red). Vertical error bars indicate the standard deviation of the mean. 
With a measure of the input angle offset $\theta_{i n}$, where we use $\theta_{i n}$ to indicate that it is not the primary input $\Delta$ value sought, where as $\Delta x_{i n}$ is. From the previously measured $R_{12}$, a statement about the $\theta_{i n}$ contribution can be made. As the following shows, the initial setup effort was worthwhile and the effect of $\theta_{i n}$ can be neglected for these data sets.

$$
\Delta x_{\text {out }}=R_{11} \Delta x_{i n}+R_{12} \Delta x_{i n}^{\prime}+R_{13} \Delta z_{\text {in }}+R_{14} \Delta \delta_{\text {in }}
$$

Equation 6.3 re-iterates the collective effect of the input vector $\Delta$ 's on $\Delta x_{\text {out }}$. Since we consider the longitudinal input parameters fixed, the last two input delta terms become zero, and then rearranging for $R_{11}$ and including the effects of the mean input angle, $\theta_{\text {in }}$ we can write:

$$
R_{11}=\frac{\Delta x_{o u t}-R_{12} \theta_{\text {in }}}{\Delta x_{\text {in }}}
$$

from which we can then solve for the fractional contribution of the 'contaminating' input angle, $\eta$ :

$$
\eta=\frac{R_{12} \theta_{\text {in }}}{\Delta x_{\text {out }}-R_{12} \theta_{\text {in }}}
$$

Thus, the input angle's contribution is the largest in $\left.\left(x_{i n}, x_{o u t}\right)\right|_{x_{j}}$ measurements where $\Delta x_{\text {out }}$ is small. Justified by the agreement with the T3D-MatLab model, we can use the previously measured matrix element $R_{12}$ and the mean measured $\theta_{i n}$ offset to calculate the fraction contribution, $\eta$ at all $T M_{110}$ Mode Cavity strengths. $\eta$ is calculated for each $\left.\left(x_{i n}, x_{\text {out }}\right)\right|_{x_{j}}$ measured point at each $T M_{110}$ Mode Cavity strength. Table 6.2 shows the calculation summary; the instance of greatest $\eta$ per cavity strength is reported. In each data set the contribution of $\theta_{\text {in }}$ is less than $1 \%$ at most; $\eta$ drops below $1 \times 10^{-3}$ for larger values of $\Delta x_{\text {out }}$.

This procedure was followed to measure the remaining elements of the $1^{\text {st }}$ column: $R_{21} \equiv$ $\partial x_{\text {out }}^{\prime} / \partial x_{\text {in }}, R_{31} \equiv \partial y_{\text {out }} / \partial x_{\text {in }}, R_{41} \equiv \partial y_{\text {out }}^{\prime} / \partial x_{\text {in }}$, and the $R_{61} \equiv \partial \delta_{\text {out }} / \partial x_{\text {in }}$ elements, with exception of the $R_{51} \equiv \partial z_{o u t} / \partial x_{i n}$ which required the streak camera. 


\begin{tabular}{|c|c|c|c|c|}
\hline $\mathbf{k}[\%]$ & $\Delta x_{\text {out }}, \sigma[\mathbf{m m}]$ & $\theta_{\text {in }}, \sigma[\mathbf{m r a d}]$ & $R_{12}, \sigma$ & $\eta$ \\
\hline \hline 0 & $1.5 \pm 0.27$ & $0.015 \pm 0.04$ & $1.02 \pm 0.02$ & 0.010 \\
25 & $0.95 \pm 0.21$ & $0.011 \pm 0.05$ & $0.64 \pm 0.04$ & 0.007 \\
50 & $-1.87 \pm 0.75$ & $0.021 \pm 0.04$ & $0.56 \pm 0.06$ & 0.006 \\
75 & $0.29 \pm 0.62$ & $-0.007 \pm 0.04$ & $-0.05 \pm 0.06$ & 0.008 \\
100 & $-0.48 \pm 0.76$ & $0.008 \pm 0.04$ & $-0.18 \pm 0.09$ & 0.003 \\
\hline
\end{tabular}

Table 6.2: Summary of input angle fluctuations, $\theta_{i n}$ and their fractional contribution, $\eta$ to the measurement of $R_{11}$

\section{Measuring Matrix Column 6}

A description of the measurement, data reduction and analysis of the $R_{66}$, matrix element, $\partial \delta_{\text {out }} / \partial \delta_{\text {in }}$ serves as an illustration of the technique utilized in measuring the $6^{\text {th }}$ column: matrix elements $R_{16} \equiv \partial x_{\text {out }} / \partial \delta_{\text {in }}, R_{26} \equiv \partial x_{\text {out }}^{\prime} / \partial \delta_{\text {in }}, R_{36} \equiv \partial y_{\text {out }} / \partial \delta_{\text {in }}$, $R_{46} \equiv \partial y_{\text {out }}^{\prime} / \partial \delta_{\text {in }}$, with the exception of the $R_{56} \equiv \partial z_{\text {out }} / \partial \delta_{\text {in }}$ which required the streak camera. The measurement of this column was also considered 'pure;' since the 9-cell gradient could be changed without affecting the other input parameters. Further, the operating phase point of the 9 -Cell was $5^{\circ}$ off crest to reduce phase-induced momentum jitter. Prior to data collection, the RF gun and 9-Cell RF controls were tuned for maximum beam stability. The input beam's momentum was measured in the straight ahead spectrometer to have a fractional rms jitter of $5 \times 10^{-4}$ or less over a 30 second period. 

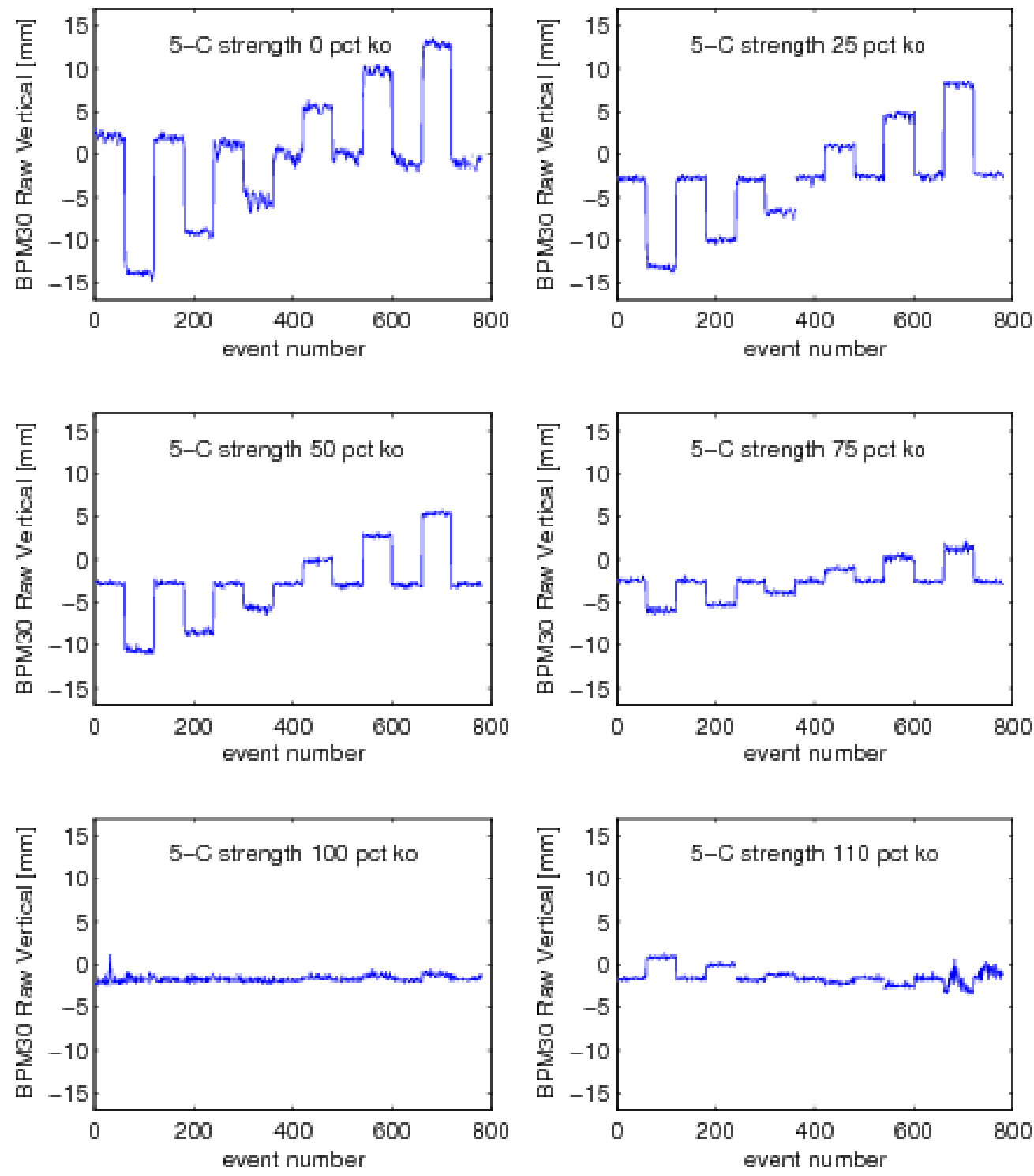

Figure 6.13: BPM30 vertical readings throughout a $\Delta \delta$ input momentum program sequence for six differing $T M_{110}$ Mode Cavity strengths. Note over-compensation at $110 \% k_{o}$ 
The change in output momentum, $\Delta \delta_{\text {out }}$ was measured by taking advantage of the placement of BPM30 in the dispersive section after the EEX spectrometer. It should be made clear that the defined transport matrix ends at BPM28, however the momentum information is obtained at BPM30. Assuming no significant sources of energy gain or loss between BPM28 and BPM30, the change in momentum at BPM28 was inferred by measuring the change in momentum at BPM30. The assumption of zero energy loss may not be correct, as coherent synchrotron radiation (CSR) may reduce the momentum. However, since this is a $\Delta$ measurement the pedestal of the CSR loss could not be measured. The vertical dispersion at BPM30, $D_{y, 30}$ was measured to be $865 \pm 10 \mathrm{~mm}$, thus the change in momentum is calculated from Equation 6.6:

$$
\Delta \delta_{\text {out }}=\frac{\Delta y_{\text {out }, 30}}{D_{y, 30}}
$$

A control script was written to step the 9 -Cell gradient through a $\pm 3 \%$ range in $1 \%$ increments. Accounting for the $4 \mathrm{MeV}$ pedestal gradient of the RF Gun, this is an absolute range of $\pm 2.17 \%$ in $0.72 \%$ increments. The energy step calibration was first verified in the straight ahead spectrometer section using BPM8 located after the spectrometer with horizontal dispersion of $240 \mathrm{~mm}$.

The nominal $14.3 \mathrm{MeV}$ beam was established through the EEX beamline, and data collection started by recording the standard nominal reference BPM beam events. The control script began sequencing through the 9-Cell momentum input offsets, $\Delta \delta_{i n}$ while recording $60 \mathrm{BPM}$ events per $\Delta \delta_{i n}$ offset. After each $\Delta \delta_{i n}$ offset, the 9-Cell gradient was returned to the nominal value for another sixty beam events. This was repeated for the five standard $T M_{110}$ Mode Cavity strengths. An additional measurement at the Klystron's maximum capacity, corresponding to $110 \%$ of $k_{o}$ was performed, where an overcompensation of the momentum offset was observed. The raw BPM30 vertical data is shown in Figure 6.13. The suppression of output momentum is dramatic; this reduction is another indication of emittance exchange.

The data reduction and analysis of the $6^{\text {th }}$ column is similar to the analysis of the $2^{\text {nd }}$ column. Since there were no hysteresis effects in this measurement, the background was 


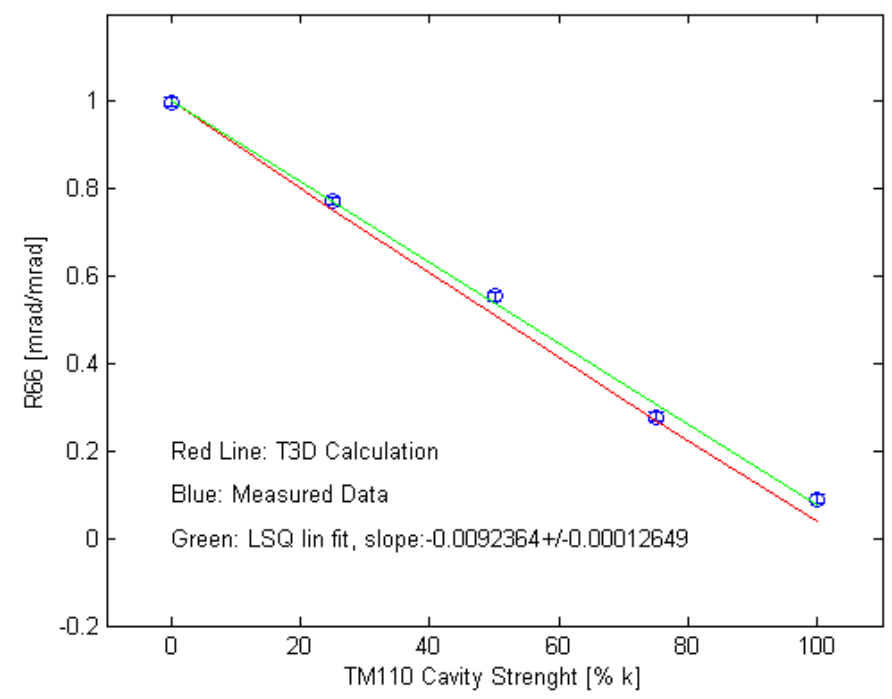

Figure 6.14: Measurement of $R_{66}$ evolution with the $T M_{110}$ Mode Cavity strength. Vertical error bars indicate standard deviation of the mean. A T3D calculation is shown for comparison.

calculated as the mean of the nominal inputs before and and the offset data, thus accounting for any linear drift during the sequence run. The $R_{66}$ evolution as a function of $T M_{110}$ Mode Cavity strength is plotted in Figure 6.14. For comparison, the T3D calculated values are plotted along with a linear least squares fit of the data. Good agreement is seen.

\section{Measuring Matrix Column 5}

We conclude the discussion of the BPM-based matrix element measurements with a description of an element in $5^{\text {th }}$ column. The $5^{\text {th }}$ column required the ability to longitudinally change the incident bunch's time of arrival with respect to the synchronous bunch. This was done by changing the the UV Laser fire time, the phase of the RF Gun, and the Phase of the 9-Cell in all concert - a herculean effort. A simpler option availed. Since all of the EEX beamline optics are static, it is equivalent to change only the phase, $\Delta \phi$ in degrees, of the $T M_{110}$ Mode Cavity to effect a time of arrival difference, thus longitudinal offset from the reference bunch. This is demonstrated in Equation 6.7:

$$
\Delta z_{i n} \equiv \frac{c \Delta \phi}{f_{o} 360^{\circ}}
$$



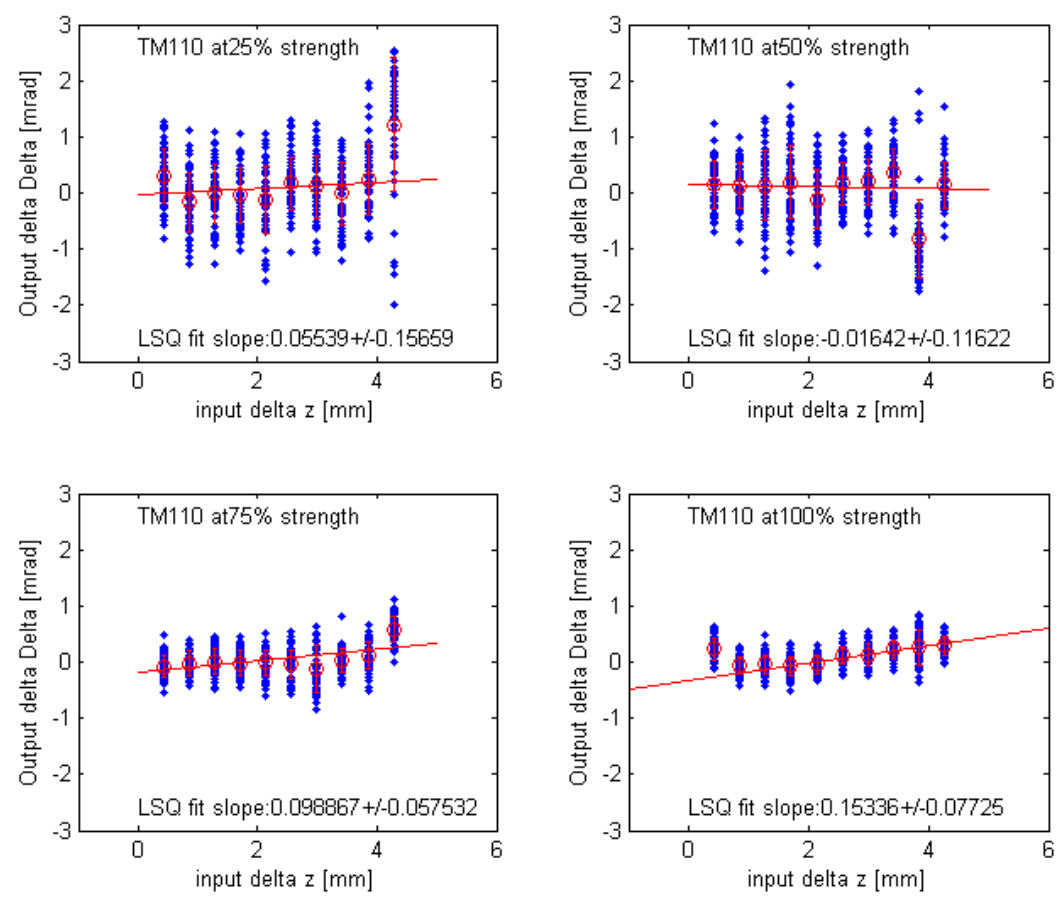

Figure 6.15: $R_{65}\left(R_{43}\right.$ in $4 \times 4$ presentation) analysis of the four standard $\mathrm{TM}_{110}$ cavity on states. Vertical error bars indicate standard deviation of the mean. The red line is a weighted least squares fit to the mean values.

I will illustrate the measurement of the $R_{65}$ element, $\partial \delta_{\text {out }} / \partial z_{i n}$. This serves to describe the technique used for the entire $5^{\text {th }}$ column, except for the $R_{55}$ which is addressed in the streak camera measurement section. The $R_{65}$ element, $\partial \delta_{\text {out }} / \partial z_{\text {in }}$, is of particular interest as it is the on-diagonal sub-block element that arises from the finite length $T M_{110}$ Mode Cavity previously calculated in Chapter 3 . The change in output momentum was measured by the procedure outlined in Section 6.4.1, where use is made of BPM30 that is located in the dispersive section after the EEX spectrometer.

The $T M_{110}$ Mode Cavity was setup for the nominal EEX operating phase, and sixty background BPM beam events were recorded. A control script sequenced the $T M_{110}$ Mode Cavity phase in $2^{\circ}$ increments. The range was $\pm 10^{\circ}$, resulting in a $\Delta z_{\text {in }}$ range of \pm 2.1 mm. The cavity phase was returned to the nominal EEX operating phase after each offset. The analysis follows the procedure used for the $R_{12}$ measurement with the exception that the mean of the nominal events before and after phase offset are used as the background value, since this procedure did not suffer from hysteresis. This accounts for any linear drifts 


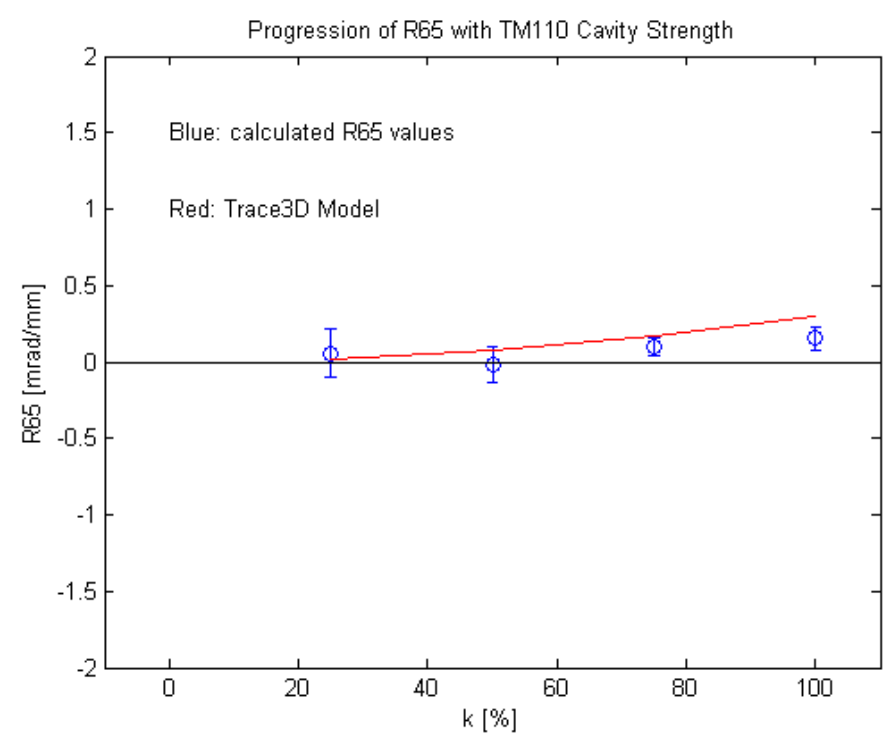

Figure 6.16: The evolution of the $R_{65}$ ( $R_{43}$ in $4 \times 4$ presentation) with $T M_{110}$ Mode Cavity strength. Vertical error bars indicate standard deviation of the mean.

during the measurement.

\subsubsection{Measuring Difference Orbits Using the Streak Camera}

Measuring the EEX matrix's $5^{\text {th }}$ row of elements, the dependence of longitudinal output position offset on the input parameters, $\partial z_{\text {out }} / \partial \Delta x, x^{\prime}, \ldots, \delta_{\text {in }}$, can be completed by measuring changes in beam arrival times. Since the $14.3 \mathrm{MeV}$ electron beam is traveling at the speed of light, a non-synchronous bunch longitudinally offset by $\Delta z$ will differ in arrival at a longitudinal reference point by $\Delta t=\frac{\Delta z}{c}$ with respect to the synchronous bunch. The Hamamatsu H6880 streak camera was employed to measure such changes in the electron bunch time of arrival on the picosecond time scale. The streak camera utilized the prompt optical transistion radiation (OTR) light generated at X24 for the longitudinal difference orbit measurements. The drift between BPM28 and X24 does not effect the arrival time differences.

Unlike the BPM measurements, data acquisition with the streak camera is manually intensive, requiring a minimum of a two-person data gathering team. The duties of the two-person team were assigned as to maximize expediency in both gathering and recording of the data; the first individual operated the streak camera controls while the second ad- 

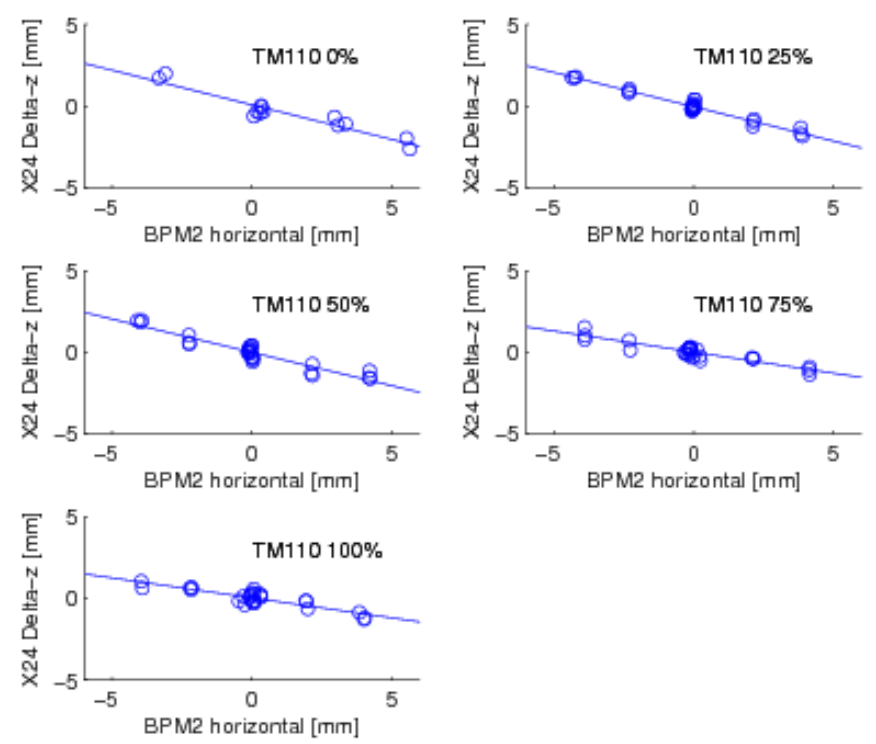

Figure 6.17: Measuring $R_{51}$ : streak camera measurement of $\Delta z$ at X24 against input (BPM2) horizontal offset at increasing $T M_{110}$ Mode Cavity strengths.

justed the desired input beam parameters, the $T M_{110}$ Mode Cavity settings and recorded the data. Due to a frequent loss of the internal streak camera phase synchronization it was imperative to use the iterative delta measurement technique with prior- and postnominal background measurement while obtaining the complete data set in the shortest time possible. A measurement of five streak measurements per $T M_{110}$ Mode Cavity setting was a compromise between gathering statistics and the mean time between streak camera operations failure.

Examples of streak camera time difference data, from the measurements of $R_{51} \equiv$ $\partial z_{\text {out }} / \partial x_{\text {in }}$, and $R_{52} \equiv \partial z_{\text {out }} / \partial z_{\text {in }}^{\prime}$ are plotted in Figures 6.17 and 6.19 respectively. In every streak camera based matrix element measurement, the output mean and variance is calculated for each input and weighted linear least squares fit is performed.

A quick check verified that $R_{53} \equiv \partial z_{\text {out }} / \partial y_{\text {in }}$ and $R_{54} \equiv \partial z_{\text {out }} / \partial y_{\text {in }}^{\prime}$, the vertical offset and angle dependencies respectively, had no measurable change as a function of $T M_{110}$ Mode Cavity strength.

Measuring the $R_{55}$ element utilized the previously described technique of affecting a change in electron bunch time of arrival by adjusting the $T M_{110}$ Mode Cavity phase. A 


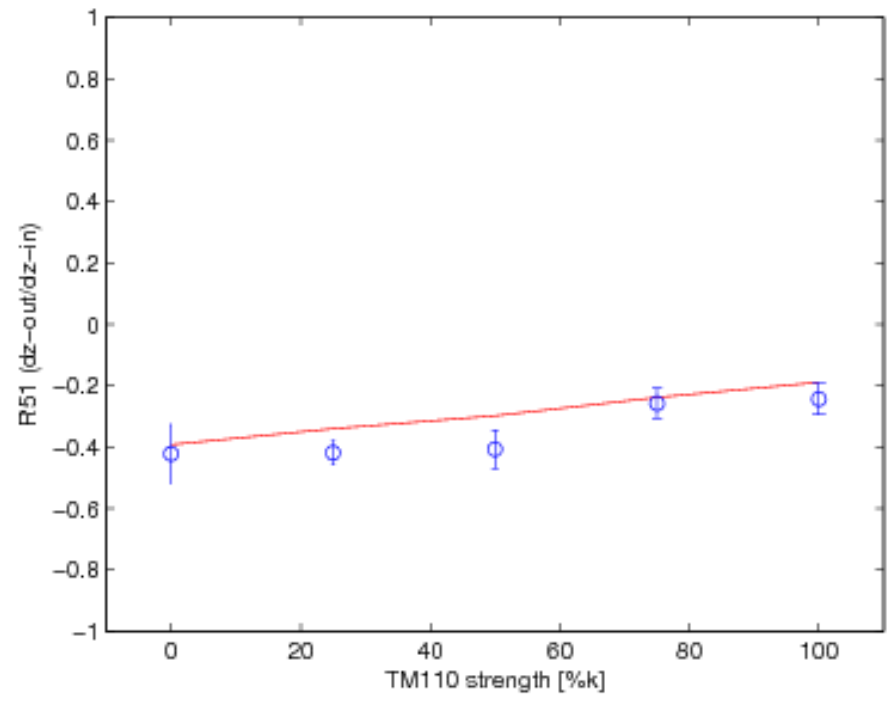

Figure 6.18: Measuring $R_{51}$ : Evolution of $R_{51}$ as a function of $T M_{110}$ Mode Cavity strength. Vertical error bars indicate standard deviation of the mean. The red line is a T3D calculation for comparison.
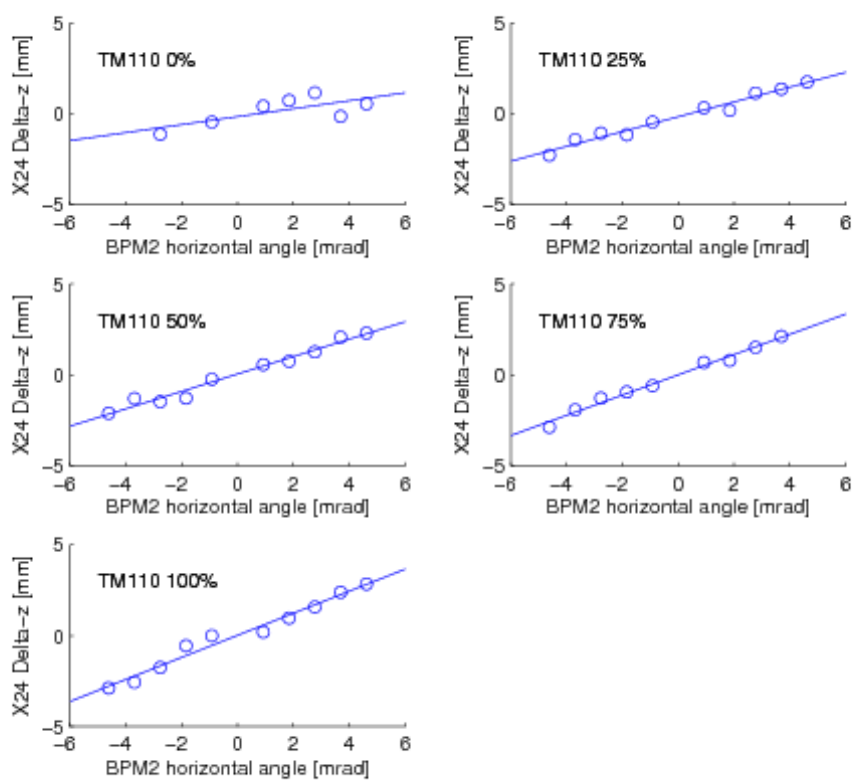

Figure 6.19: Measuring $R_{52}$ : streak camera measurement of $\Delta z$ at X24 against input horizontal angle offset for increasing $T M_{110}$ Mode Cavity strengths. The blue line is a linear fit to the data points 


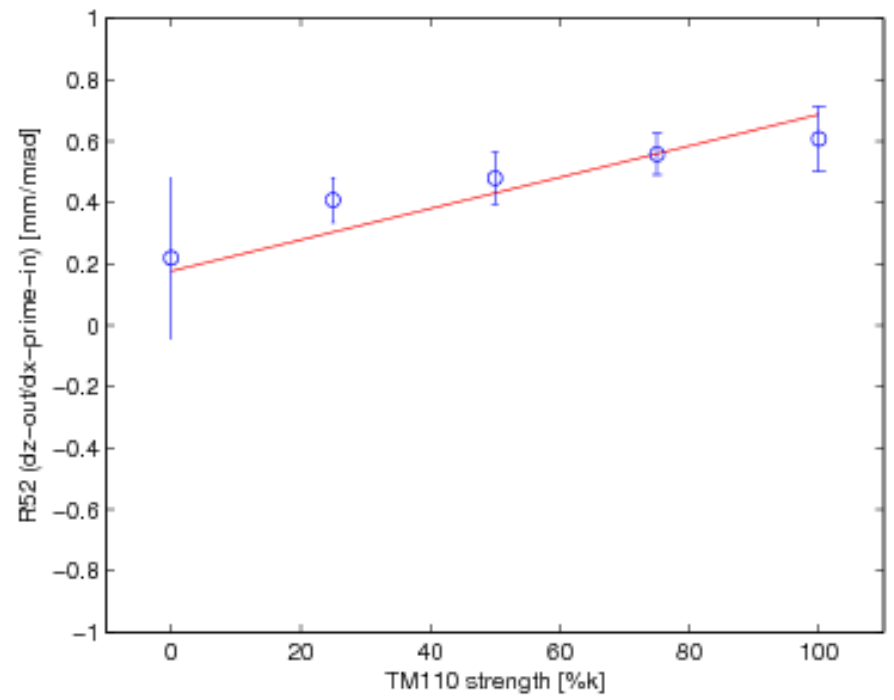

Figure 6.20: The evolution of the $R_{52}$ with the cavity strength. The red line is the expected value from the T3D-MatLab calculations.

complication arose because the cavity phase was changed rather than the arrival time. Since the change in arrival time at the X24 measurement location is expected to be inversely proportional to the cavity strength, to obtain the $R_{55}$ element for a given $T M_{110}$ Mode Cavity strength the following formula was used.

$$
R_{55}=1-\frac{\left(\frac{c \Delta \phi}{f_{0} 360^{\circ}}\right)}{\Delta z_{\text {out }}}
$$

where $f_{0}$ is $3.9 \mathrm{GHz}$, and $\Delta \phi$ is the $T M_{110}$ Mode Cavity phase offset in degrees from the ideal EEX phase.

The measurement of the $R_{56} \equiv \partial z_{\text {out }} / \partial \delta_{\text {in }}$ element was straight forward. The gradient of the 9-Cell superconducting cavity was again sequenced while the resulting change in bunch arrival was measured with the streak camera. 


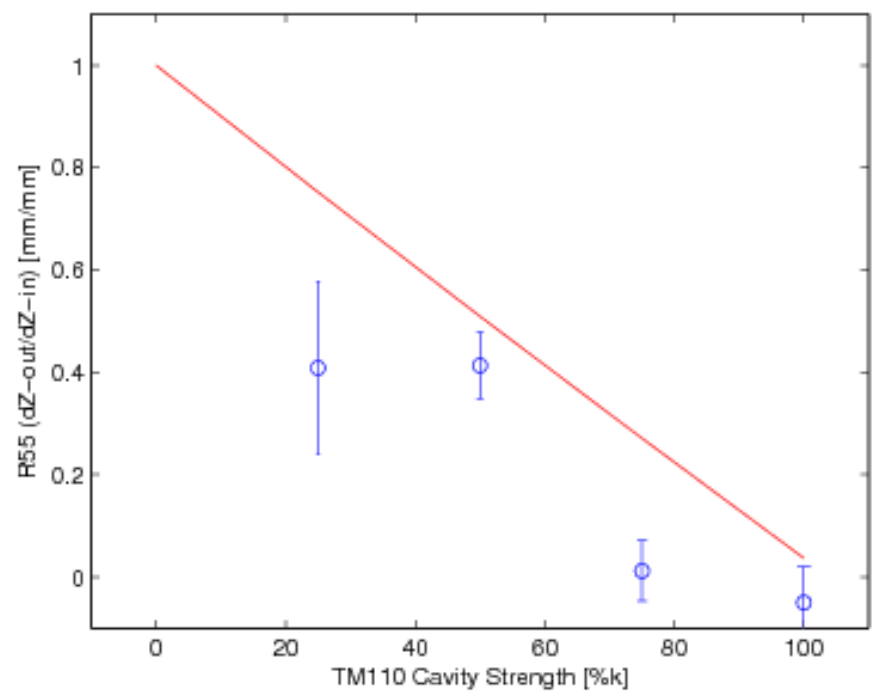

Figure 6.21: The evolution of the $R_{55}$ with the cavity strength. The red line is the expected value from the T3D-MatLab calculations.

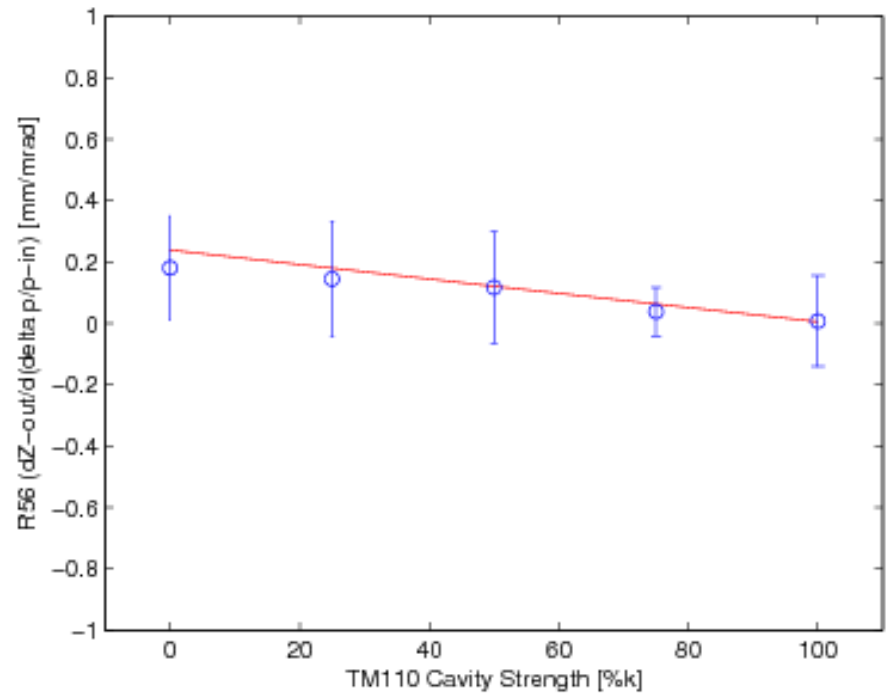

Figure 6.22: A measurement of the momentum compaction factor, $R_{56}$, as a function of cavity strength. 

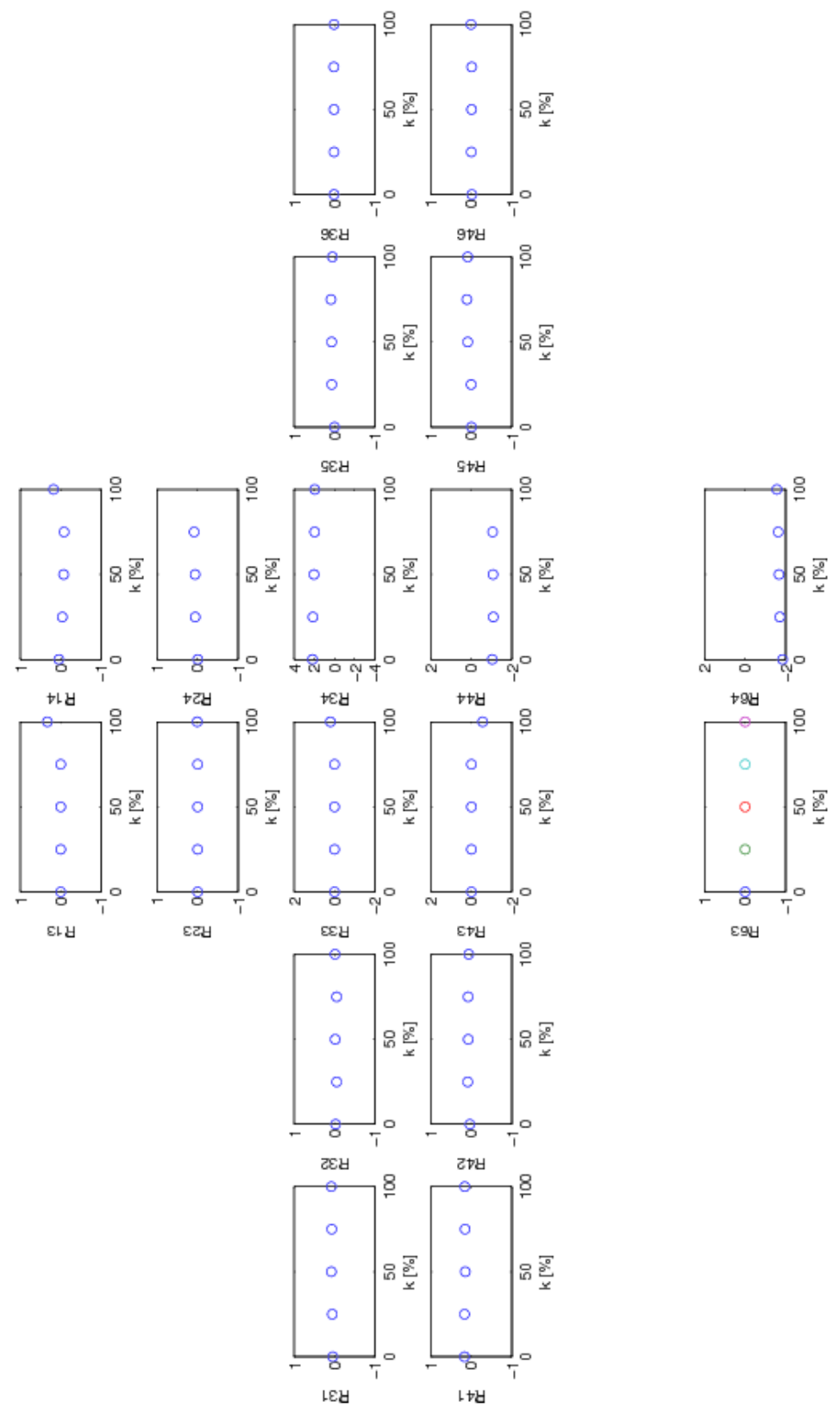

Figure 6.23: A survey measurement of the vertical matrix elements to verify inactivity. (note image is rotated $90^{\circ}$ for viewing) 


\subsection{Survey Measurement of Columns 3 \& 4 and Rows $3 \& 4$}

A quick survey measurement of the EEX matrix columns 3 and 4 were performed as well as the matrix rows 3 and 4 . Dedicated beam time for a measurement of $R_{53}$ and $R_{54}$ beyond the quick check was not possible due to the required time consuming streak camera method. As expected, the response of the vertical based elements showed no measurable correlation with the $T M_{110}$ Mode Cavity state, thus their further pursuit was deemed unnecessary. Figure 6.23 shows the cursory analysis.

\subsection{Compilation of Matrix Measurements}

The goal of verifying the $6 \times 6$ emittance exchange transport matrix was accomplished; the preceding sections describe the varied measurement and analysis techniques for the individual matrix elements. In bringing the individual matrix elements together in the $4 \times 4, x-z$ plane format, the desired emittance exchange transport matrix form becomes evident as the $T M_{110}$ Mode Cavity strength is ramped to full strength. The final figure of this chapter, Figure 6.24, compares the measured matrix elements against the previously discussed T3D-MatLab beamline model with increasing cavity strength. The circles are the measured quantities, and the solid red lines reflect the model.

Lastly, Equation 6.9 lists the measured numeric values of the $k_{o}=100 \%$ case, i.e. the Emittance Exchange values. We make a numerical comparison between the T3D-MatLab calculated EEX Matrix Elements using the 5-cell pillbox cavity model, Equation 6.10, and the measured EEX matrix Elements, Equation 6.9 for the EEX, $k=k_{o}$ state.

The measured EEX values are:

$$
M_{E E X_{\text {measured }}}=\left(\begin{array}{cccc}
-0.02 \pm 0.059 & -0.23 \pm 0.089 & 4.75 \pm 0.350 & 0.40 \pm 0.003 \\
-0.02 \pm 0.167 & 0.11 \pm 0.041 & -0.02 \pm 0.540 & 0.21 \pm 0.002 \\
0.23 \pm 0.051 & 0.63 \pm 0.107 & -0.21 \pm 0.312 & 0.00 \pm 0.148 \\
-0.09 \pm 0.017 & 4.89 \pm 0.047 & 0.13 \pm 0.080 & 0.08 \pm 0.010
\end{array}\right)
$$

Next, the T3D-MatLab EEX matrix element calculated values, repeated from Equation 
3.45:

$$
M_{E E X_{\text {calc'd }}\left(k_{o}\right)}=\left(\begin{array}{cccc}
-0.013 & -0.008 & 4.832 & 0.345 \\
0.014 & 0.006 & -0.642 & 0.161 \\
0.190 & 0.687 & 0.037 & 0.004 \\
-0.058 & 5.059 & 0.300 & 0.035
\end{array}\right)
$$

Equation 6.11 shows the difference of these two sets:

$$
M_{\text {difference }}=\left(\begin{array}{cccc}
0.03 \pm 0.059 & 0.17 \pm 0.089 & 0.34 \pm 0.350 & -0.02 \pm 0.003 \\
0.02 \pm 0.167 & -0.07 \pm 0.041 & -0.61 \pm 0.540 & -0.01 \pm 0.002 \\
-0.05 \pm 0.051 & 0.08 \pm 0.107 & 0.13 \pm 0.312 & -0.01 \pm 0.148 \\
0.03 \pm 0.017 & 0.49 \pm 0.047 & 0.15 \pm 0.080 & -0.06 \pm 0.010
\end{array}\right)
$$

Overall, there is good agreement between the calculated and the measured EEX matrices. The most significant discrepancies are with elements $R_{23} \equiv \partial x_{\text {out }}^{\prime} / \partial z_{\text {in }}$ being a factor of 2 two large and with $R_{43} \equiv \partial \delta_{\text {out }} / \partial z_{i n}$. From the $R_{23}$ evolution plot of Figure 6.24 , it is apparent that the final data point, at $k_{o}$, is uncharacteristic of the trend set by the previous three data points. This behavior is consistent with a large pressure jump in the $T M_{110}$ Mode Cavity $l N_{2}$ bath. Unfortunately this element was not remeasured, and future future plans should consider remeasurement. The $R_{43}$ element displayed a turn on evolution, but not at the expected rate, leaving the final value about one half that anticipated. $R_{43}$ is biggest non-zero term in the on-diagonal sub-block of the $M_{E E X}$ transport matrix, thus it is responsible for the transport optics' contribution to the emittance dilution. Future research should revisit this element to determine why it is significantly lower than expected. A ratio inspection of the elements whose values become small are seen to be sensitive to the $T M_{110}$ Mode Cavity strength, thus a slight change in $k$ effects the ratio greatly. 

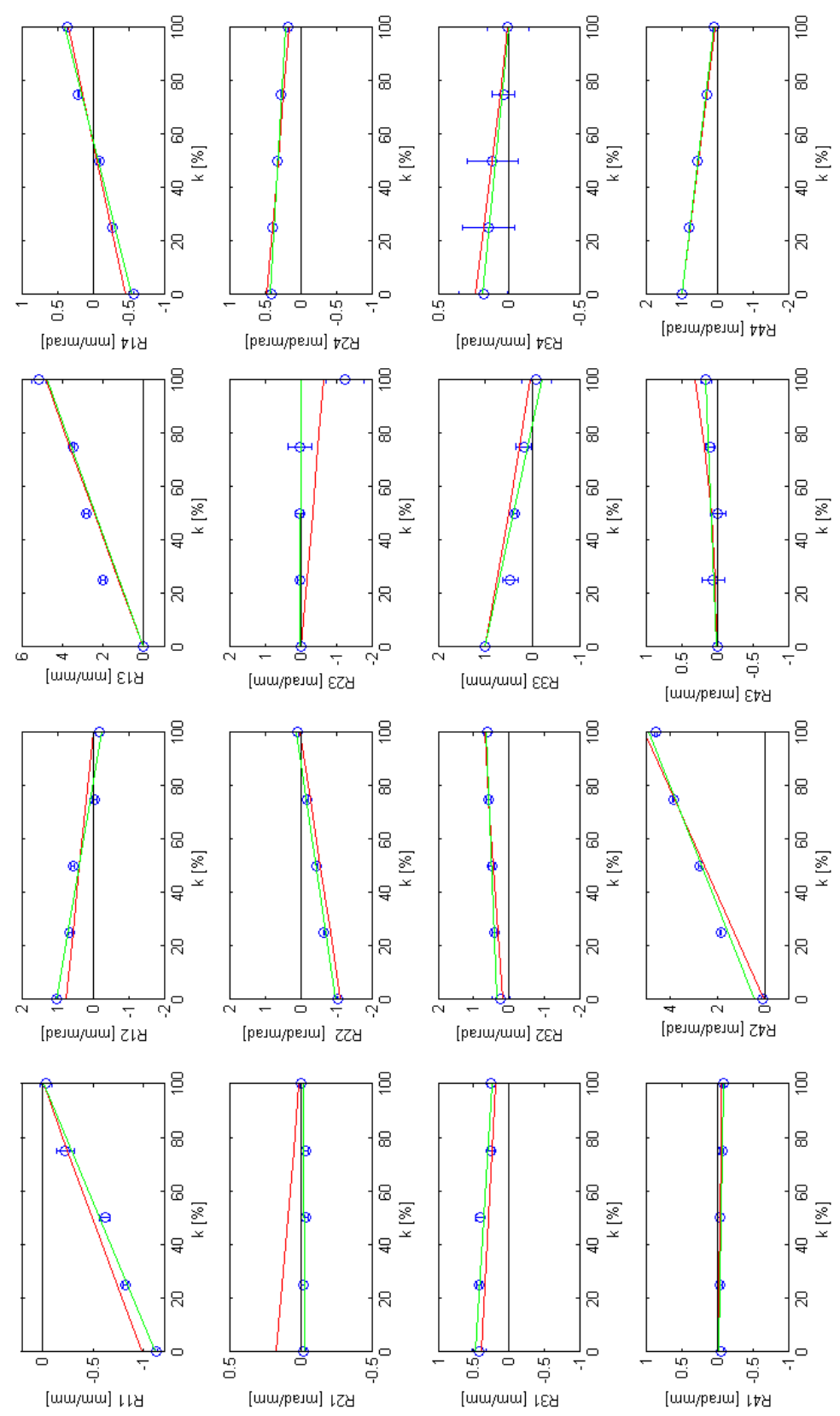

Figure 6.24: The evolution of the $4 \mathrm{x} 4$ (horizontal and longitudinal) EEX transport matrix as a function of $T M_{110}$ Mode Cavity strength (note image is rotated $90^{\circ}$ for viewing) 


\section{Chapter 7 Initial Observation of Emittance Exchange}

After completing a measure of the emittance exchange transport optics, several direct emittance exchange measurements were performed. The observed emittance exchange utilized a 14.3 MeV $250 \mathrm{pC}$ electron beam generated by the A0 Photoinjector. A bunch charge of $250 \mathrm{pC}$ was chosen as a compromise between diagnostic requirements and space charge effects. Calculations by H. Edwards [68] provided an initial starting point for input beam conditions. The beam parameters at the observed emittance exchange point were empirically found by searching near the suggested input values for a minimum output bunch length-momentum spread product, $\sigma_{z} \cdot \sigma_{\delta}$. During the EEX experiments, sub-picosecond bunch lengths, as well as very small energy spreads, $\approx 10 \mathrm{keV}$, could be obtained; however, not simultaneously. Effects such as space charge and coherent synchrotron radiation may be significant sources of emittance dilution and will be the focus of future EEX studies at the Photoinjector. This chapter discusses the conditions under which the first transverse to longitudinal emittance exchanges has been observed and compares the results against a simple calculation.

For reproducibility between experimental runs, all iron based magnets were degaussed and restored to nominal operating conditions from the control system's Save \& Restore file. A degauss was performed at the beginning of every experimental run, and as needed as a result of experimental operations.

\subsection{Input Beam Parameters}

A large ratio of the input transverse to longitudinal emittance is desirable for contrasting the input to output exchange. However, transverse output diagnostics, such as the screen 
size at X23, placed limitations on the input longitudinal emittance. For instance, since the input longitudinal emittance is transferred to the transverse output emittance, too large an $\epsilon_{z, \text { in }}$ will partially manifest itself as a transverse spot too large to view on the X23 viewing screen. To make reliable measurements, we had to ensure that the transverse spot was fully contained on the screen, thereby limiting the input longitudinal emittance. The converse also applies. In principle, a smaller transverse input emittance becomes a small output longitudinal emittance. Indeed, extremely short bunch lengths and energy spreads were measured during the experiments. Measured input emittance conditions in the approximate ratio of 5:5:21 $\left(\epsilon_{x}, \epsilon_{y}, \epsilon_{z}\right)$ were repeatedly measured under a transverse to longitudinal emittance exchange.

At a $250 \mathrm{pC}$ bunch charge, a scan of the superconducting 9-cell cavity phase identified a minimum energy spread at 28 degrees off crest, measured in the straight ahead spectrometer at XS3, Figure 7.1. A 9-cell phase scan, with the beam propagating through the EEX beamline with the $T M_{110}$ Mode Cavity off, indicated maximum bunch compression at the exit of the exchange beamline. Since the dispersion at the cavity is approximately a $50 \%$ of the dispersion at the exchanger exit, the bunch compression was also 50 percent the cavity location. Since we were operating at $250 \mathrm{pC}$, and the laser pulse duration was fixed, and thus the electron bunch length, the choice of 9-Cell operating phase is the only 'knob' remaining to adjust for minimum energy spread. After the minimum energy spread operating point was empirically located, attention was turned to the transverse emittances. A solenoid scan was performed to obtain a minimum projected transverse emittance at X3 utilizing the slit method, typically $5 \mathrm{~mm} . \mathrm{mrad}$. The beam was then restored to the straight ahead spectrometer, where quadrupoles Q1AX14 through Q3AX14 were used to minimize the horizontal $\beta$-function's contribution to the spot width $\sigma_{x, \beta}$ at XS3. The bunch energy spread, $\delta=\sigma_{p} / p_{o}$, was calculated from the remaining horizontal spot size, $\sigma_{x}$, divided by the dispersion, $D_{X S 3}=324 \pm 10 \mathrm{~mm}$, at the viewing screen.

$$
\sigma_{p}=p_{o} \frac{\sigma_{x}}{D_{X S 3}}
$$

With the input beam parameters set, the beam was propagated through the EEX beam- 


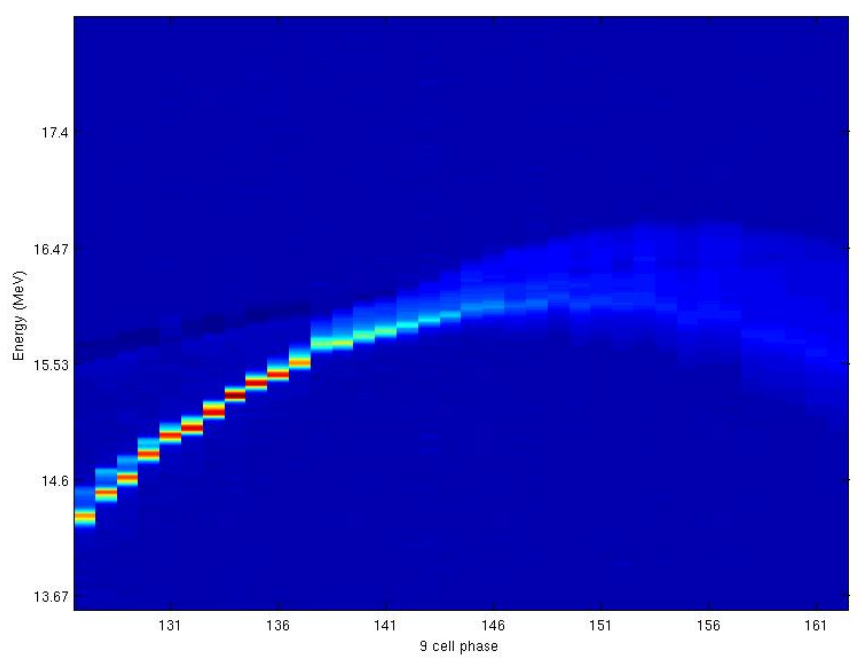

Figure 7.1: Energy and energy spread as a function of the 9-Cell Phase

line; initially with the $T M_{110}$ Mode Cavity off to ensure that the proper reference obit had been established. The $T M_{110}$ Mode Cavity was then energized to the EEX $k_{o}$ gradient and phase (see Appendix $\mathrm{C}$ for details in the cavity operational setup).

\subsection{Input Quadrupole Scans}

As previously mentioned, we searched for input transverse conditions that yielded the minimum output $\sigma_{z} \cdot \sigma_{\delta}$ product, this we take to be the projected output longitudinal emittance, $\epsilon_{z}$. This was accomplished by adjusting the 'input quadrupoles' Q1AX05, and Q2AX06.

\subsubsection{Pyroelectric-Quad Scans}

With Q1AX03 held at a fixed value of -0.23 A, quadrupoles Q1AX05 and Q2AX06 were scanned while recording the raw voltages from the Martin-Puplett (MP) interferometer pyroelectric detectors. During these scans the interferometer was not operating, thus we were only making use of its pyroelectric sensors' ability to measure coherent transition radiation (CTR) intensity. As describe in Chapter 4, the CTR signal is inversely proportional to bunch length, thereby allowing an output bunch length map to be generated. We have termed this use of the interferometers sensors as a 'pyro-scan.' This technique is only a 


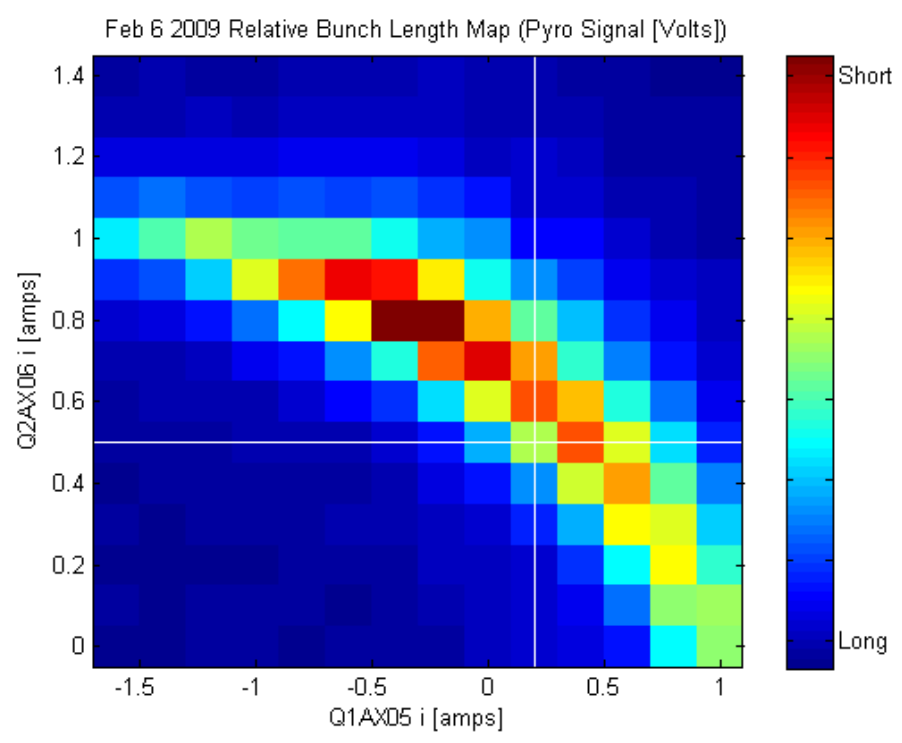

Figure 7.2: An output relative bunch length map against input quadrupoles Q1AX05 and Q2AX06 current settings - Feb 62009 data run. The white cross hairs indicate the EEX operating point of Feb 62009.

relative measurement, as it is difficult to calibrate a single pyroelectric detector against bunch length. The benefit of a pyro-scan is that it is a quick measurement which identifies a region of interest to be revisited with the calibrated interferometer or streak camera. As displayed in Figure 7.2, there is a clear valley of minimum bunch length.

\subsection{Momentum-Quadrupole Scan}

After the completion of a pyro-scan, but before a calibrated interferometer or streak camera bunch length measurement, an output momentum-spread scan was performed. Again, with Q1AX03 held at the fixed value, quadrupoles Q1AX05 and Q2AX06 were scanned while digital images of the XS4 spectrometer screen were taken. Before commencing the momentum scan output Q1AX23, Q2AX23, Q3AX23 quadrupoles were set to give an approximate minimum vertical spot on the XS4 screen; the output quads were not changed during the scan. As part of the automated momentum scan the laser shutter is closed and a 'background' image is recorded and subtracted. A fitting routine determines the vertical rms spot size, that allowed an energy spread to be calculated using an equation similar to Equation 7.1, with the dispersion value of $944 \pm 10 \mathrm{~mm}$ at the XS4 viewing screen. Figure 


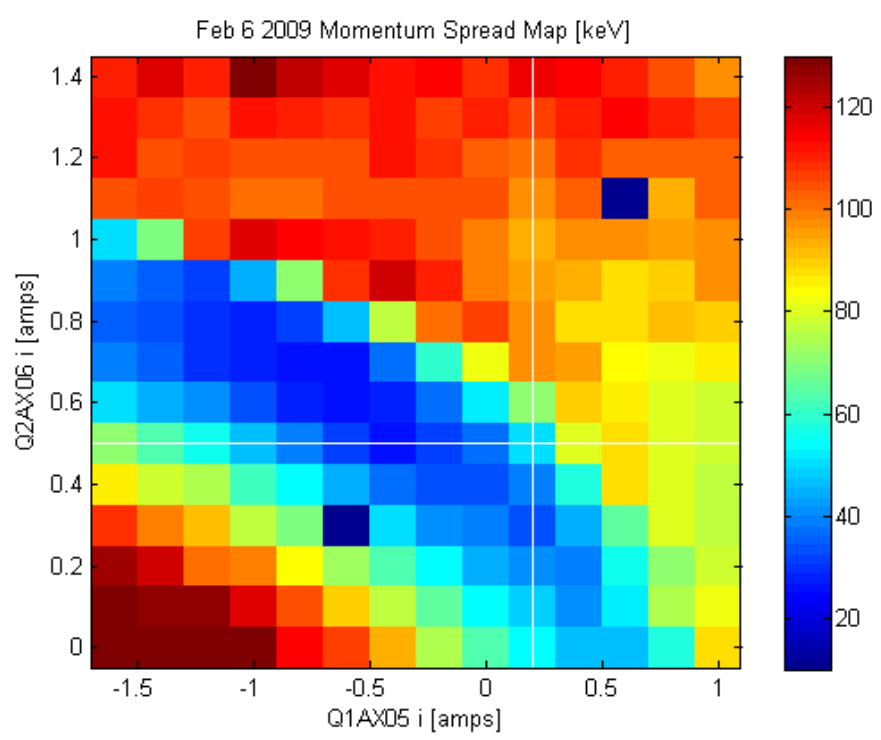

Figure 7.3: An output momentum map against input quadrupoles Q1AX05 and Q2AX06 current settings - Feb 62009 data run. The white cross hairs indicate the EEX operating point of Feb 62009.

7.3 shows a map of the output energy and indicates a valley of minimum energy spread. However, note that the bunch length valley and the momentum spread valley do not overlap.

With the pyro-scan and momentum spread maps, we identify a region of interest. For a fixed Q1AX05 value, we performed a scan along Q2AX06 that covered both the energy spread and bunch length minimums and the region in between. In addition to performing calibrated interferometer scans, for each Q2AX06 setting, a momentum spread measurement was also made. The 'EEX output quadrupoles', Q1AX23 through Q3AX23, were used to minimize the vertical spot on the XS4 viewing screen, thus minimizing the $\beta$-function contribution. The output energy spreads were then determined from the XS4 rms vertical spot size. For very small energy spreads, the $\beta$-functions' contribution may not be negligible. This is presently being studied.

It is instructive to normalize both the momentum spread and the reciprocal of the pyroscan maps to a maximum value of 1 and then map their product. Figure 7.4 shows the result indicating a region of minimum output bunch length energy spread product. We find the minimum $\sigma_{z} \cdot \sigma_{\delta}$ and take this to be the worst case output longitudinal emittance as we are neglecting the correlation $\sigma_{z \delta}$, remembering that the longitudinal rms emittance is $\epsilon_{z}=\sqrt{\sigma_{z}^{2} \sigma_{\delta}^{2}-\sigma_{z \delta}^{2}}$ 


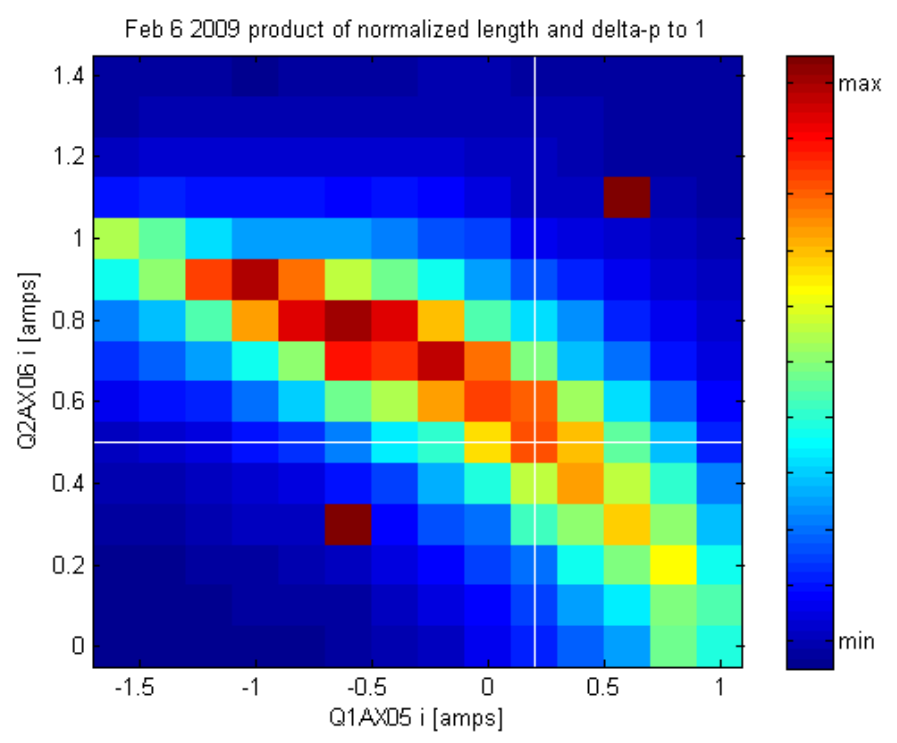

Figure 7.4: A map of the product between $1 / \sigma_{\delta}$ normalized to 1 and $1 / \sigma_{z}$ also normalized to 1 from the Feb 62009 data. The white cross hairs indicate the EEX operating point of Feb 62009.

\subsection{Calibrated Output Projected Longitudinal Emittance}

Finally a calibrated bunch length measurement along the narrowed region of interest was performed. Quadrupole Q2AX06 was scanned in 0.1 ampere steps, e.g. along the vertical line of the white cross hairs in Figures 7.2 and 7.3. At each increment, a calibrated interferometer scan was taken. Before moving onto the next quadrupole value, another momentum spread measurement was also taken. For each of the momentum spread measurements of this sequence, the output quadrupoles were adjusted for a minimum vertical spot size and then a background subtracted image was recorded. The product of the output longitudinal parameters were then plotted as a function Q2AX06 strength. Figure 7.5 plots the output bunch length and output energy spread. Their product, the projected longitudinal emittance, is normalized to $\gamma=28$, and is plotted in Figure 7.6. I reiterate, that being unable to determine the time-energy correlation, the product is taken to be the projected (worst case) value for the output longitudinal emittance. 


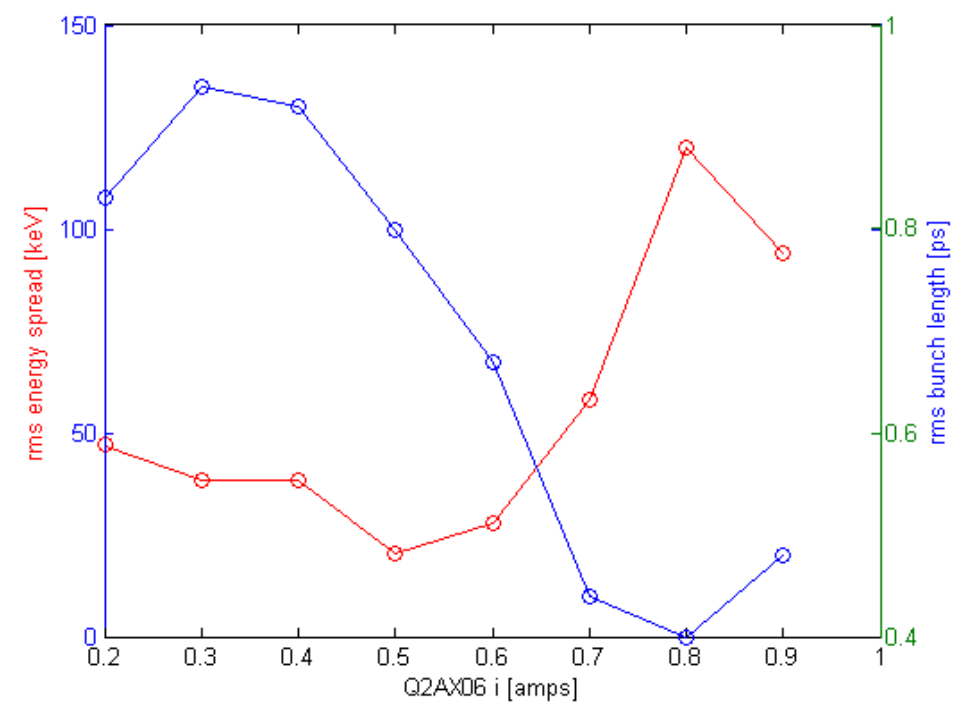

Figure 7.5: Calibrated bunch length and energy spread measured during Q2AX06 scan.

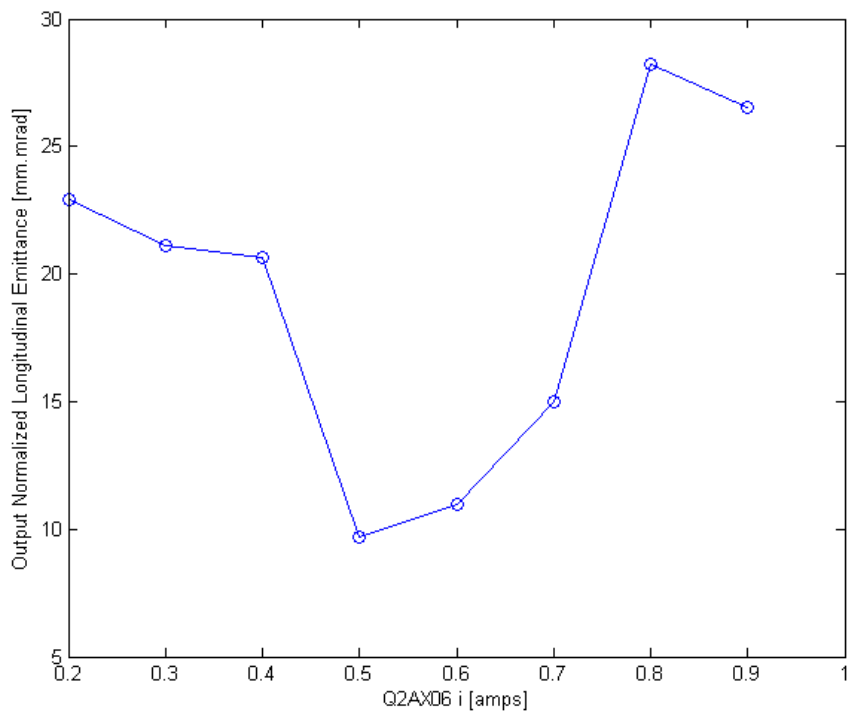

Figure 7.6: Finding minimum $\sigma_{\delta_{\text {out }}} \cdot \sigma_{z_{\text {out }}}$ product, taken to be worst case projected ouput longitudinal emittance. 


\subsection{Observed Emittance Exchange}

After the above quadrupole scan was completed, Q2AX06 was returned to the measured point of the minimum output projected longitudinal emittance. Then the transverse output projected emittance was measured at X23/X24 slit emittance measurement station. The Tables 7.1 and 7.2 are two data samples comparing the measured input and output beam conditions and their calculated normalized emittances.

\begin{tabular}{|c|c|c|c|}
\hline Coordinate & $\sigma_{i}[\mathrm{~mm}]$ & $\sigma_{i}^{\prime}[\mathrm{mrad}]$ & $\epsilon_{i, N}[\mathrm{~mm} . \mathrm{mrad}]$ \\
\hline \hline$x_{\text {in }}:$ & $0.71 \pm 0.03$ & $0.28 \pm 0.01$ & $5.59 \pm 0.27$ \\
\hline$y_{\text {in }}:$ & $0.58 \pm 0.03$ & $0.36 \pm 0.01$ & $5.89 \pm 0.24$ \\
\hline$z_{\text {in }}:$ & $0.89 \pm 0.04$ & $0.85 \pm 0.06$ & $21.4 \pm 1.49$ \\
\hline \hline$x_{\text {out }}:$ & $2.43 \pm 0.14$ & $0.32 \pm 0.03$ & $21.7 \pm 2.08$ \\
\hline$y_{\text {out }}:$ & $0.85 \pm 0.04$ & $0.37 \pm 0.02$ & $8.85 \pm 0.62$ \\
\hline$z_{\text {out }}:$ & $0.24 \pm 0.04$ & $1.44 \pm 0.07$ & $9.68 \pm 1.53$ \\
\hline
\end{tabular}

Table 7.1: Summary of emittance exchange data set of Feb 62009 taken by A0 Emittance Exchange team. Data at minimum $\epsilon_{z, \text { out }}$ determined from Q1AX05 $=0.2 \mathrm{amp}$ scan of Q2AX06. Minimum $\epsilon_{z, \text { out }}$ point at Q1AX03 $=0.23$ amps, Q1AX05=0.2 amps, Q2AX06=0.5 amps (reported emittances are normalized, $\gamma=28.0$ )

\begin{tabular}{|c|c|c|c|}
\hline Coordinate & $\sigma_{i}[\mathrm{~mm}]$ & $\sigma_{i}^{\prime}[\mathrm{mrad}]$ & $\epsilon_{i, N}[\mathrm{~mm} . \mathrm{mrad}]$ \\
\hline \hline$x_{\text {in }}:$ & $0.73 \pm 0.03$ & $0.23 \pm 0.01$ & $4.67 \pm 0.22$ \\
\hline$y_{\text {in }}:$ & $0.78 \pm 0.03$ & $0.23 \pm 0.01$ & $5.11 \pm 0.21$ \\
\hline$z_{\text {in }}:$ & $0.75 \pm 0.01$ & $1.00 \pm 0.07$ & $21.1 \pm 1.50$ \\
\hline \hline$x_{\text {out }}:$ & $2.92 \pm 0.16$ & $0.26 \pm 0.02$ & $20.8 \pm 2.00$ \\
\hline$y_{\text {out }}:$ & $0.70 \pm 0.04$ & $0.31 \pm 0.02$ & $6.00 \pm 0.42$ \\
\hline$z_{\text {out }}:$ & $0.26 \pm 0.04$ & $0.97 \pm 0.05$ & $7.06 \pm 0.43$ \\
\hline
\end{tabular}

Table 7.2: Summary of emittance exchange data set of Feb 112009 taken by A0 Emittance Exchange team. Data at minimum $\epsilon_{z, \text { out }}$ determined from Q1AX05=-0.4 amp scan of Q2AX06. Minimum $\epsilon_{z, \text { out }}$ point at Q1AX03=0.23 amps, Q1AX05=-0.4 amps, Q2AX06=0.6 amps (reported emittances are normalized, $\gamma=28.0$ )

Table 7.1 indicates that $\epsilon_{z, \text { in }}$ became $\epsilon_{x, \text { out }}$ in a 1:1 ratio, however there was a significant increase in the $\epsilon_{z, \text { out }}$ when compared to the $\epsilon_{x, i n}$, in the ratio of 1:1.7. There was also a measured increase in the projected vertical emittance, in the ratio of 1:1.5. In comparing input and output emittances of the February 11 data set, Table 7.2 reports a reduction in longitudinal emittance and a significant increase in transverse horizontal emittance. Within errors, $\epsilon_{z_{\text {in }}}$ was transported to $\epsilon_{x_{\text {out }}}$. There was about a $1: 1.5$ increase in $\epsilon_{z_{\text {out }}}$ when compared 
with $\epsilon_{z_{i n}}$. Also, there was approximately a $20 \%$ increase in the measured vertical emittance, which is not yet understood. The larger than $1: 1 \epsilon_{x_{i n}}: \epsilon_{z_{\text {out }}}$ ratio most likely stems from the combined effects of the non-ideal emittance exchange transport matrix, discussed in the next section, resolution in the measurement of $\sigma_{p}$, and as well as space charge, which will be discussed in the final Chapter.

In both the February 6 and 11 data sets, transverse emittance uncertainties are assigned from a statistical analysis of a previous, but representative, data set of September 15, 2009. During the September 152008 EEX experimental run, five measurements of each output parameter were recorded. Fractional rms uncertainties were calculated from the statistical variation of each measurement and are applied to the measured parameters of subsequent data sets.

A clear 1:1 exchange of $\epsilon_{z, \text { in }} \Leftrightarrow \epsilon_{x, \text { out }}$ is observed in the above data, however, both sets lack a preservation of $\epsilon_{x, \text { in }}$ going to $\epsilon_{z, \text { out }}$. There are at least four contributing factors to the apparent emittance growth; first the resolution of $\sigma_{p}$ which includes the vertical $\beta$-function's contribution to the spot size at the EEX spectrometer. Secondly, emittance growth from space charge; third, emittance coupling from the non-zero $R_{4,3}$ element. Finally, the inability to measure the longitudinal correlation. We can understand the last two factors by calculating the expected EEX behavior in even a simple model. The following, Section 7.6, explores the input quadrupole scans through a MatLab model and their effects on the measure output values.

\subsection{T3D MatLab Calculations}

The resulting $\sigma_{\text {out }}$ beam matrix of Equation 2.42 was used to model the quadrupole scans in MatLab. In the case where the correlation terms of the $\sigma_{\text {out }}$ matrix vanish, the determinant of the $\sigma_{z_{\text {out }}}$ sub-block is the projected emittance. However, the $\sigma_{z_{\text {out }}(1,1)}$ term is the square of the projected longitudinal bunch length, and the $\sigma_{z_{\text {out }}(2,2)}$ is the square of the projected fractional momentum spread. Equivalently, $\sigma_{x_{\text {out }}(1,1)}$ is the square of the rms transverse spot size and $\sigma_{x_{\text {out }}(2,2)}$ is the transverse rms divergence. Several interesting calculations are made from these quantities. 
We start with a description of calculation's optics setup. The 'input' section of the EEX beamline began at X3 and was assembled with three thin lens quadrupoles matrices representing Q1AX03, Q1AX05, and Q2AX06 spaced by appropriate drifts and terminated at the start of Dipole 1. The 'second half' of the beamline was a single matrix calculate by the existing T3D-MatLab model (5 cell pillbox) with the $T M_{110}$ Mode Cavity at $k=$ $100 \% k_{o}$, which began at Dipole 1 and ended at X24. The choice of X3 as the starting point, permitted input of experimentally measured and derived quantities, i.e. $\alpha_{x}, \beta_{x}, \epsilon_{x, i n}$, etc. The output choice of X24 is that it is the experimental measurement point of $\sigma_{z}$. This model used thin lens approximations for Q1AX03 through Q2AX06 of effective $106 \mathrm{~mm}$ effective length, and their current-to-gradient calibrations discussed in Section 4.3.2. During the scan, the MatLab code evaluated the total beamline matrix, then propagated the input sigma matrix from X3 through to X24. Several informative plots can be made. The output bunch length was calculated from $\sigma_{z, \text { out }}=\sqrt{\epsilon_{z, \text { out }} \beta_{z, \text { out }}}$ as square root of $\sigma_{\text {out }(3,3)}$ element. For contrasting the small bunch length, the inverse $1 / \sigma_{z, \text { out }}$ is plotted in 7.7. Cross hairs indicate the Feb 6 (white) and 11 (green) operating locations. While there was not an exact overlap, there is close agreement. Similarly, the calculated inverse $1 / \sigma_{\delta_{\text {out }}}$ is plotted in Figure 7.8. Again, while there is not direct overlap, the model does show consistent behavior with the measured $\sigma_{\delta_{\text {out }}}$. This model does not include the defocusing effects of space charge. It is interesting to note that in both the measured as well as the calculated quadrupole scans, the valleys of minimum $\sigma_{z, \text { out }}$ and $\sigma_{\delta_{\text {out }}}$ do not overlap. 


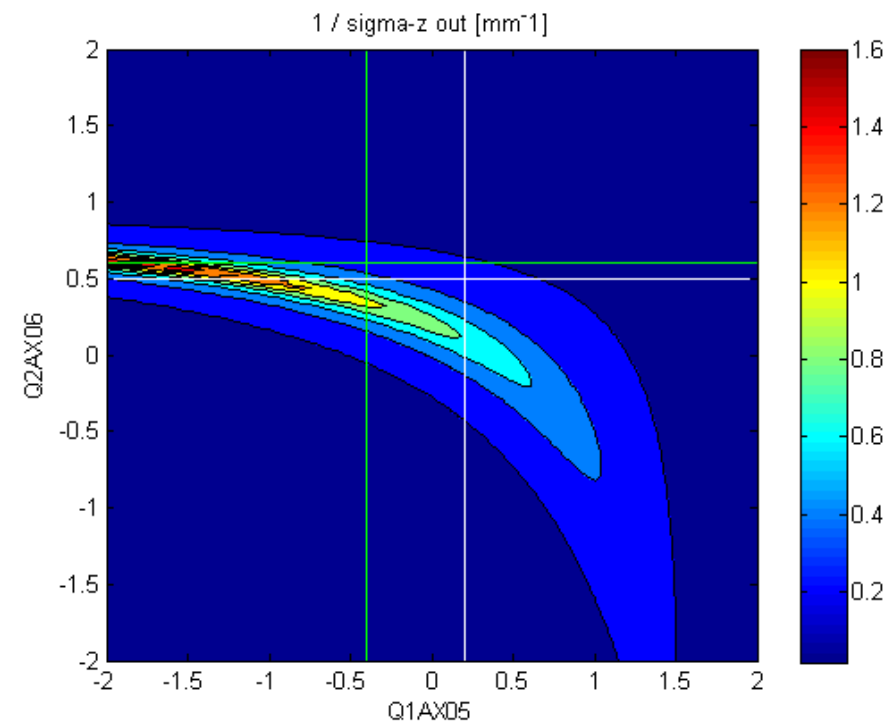

Figure 7.7: T3D MatLab Calculated $\sigma_{z}$ Q1AX05-Q2AX06 quadrupole scan, plotting 1/ $\sigma_{z}$ to contrast the short bunch length. The white cross hairs indicate the Feb 62009 data set operating point, while the green cross hairs indicate the Feb 112009.

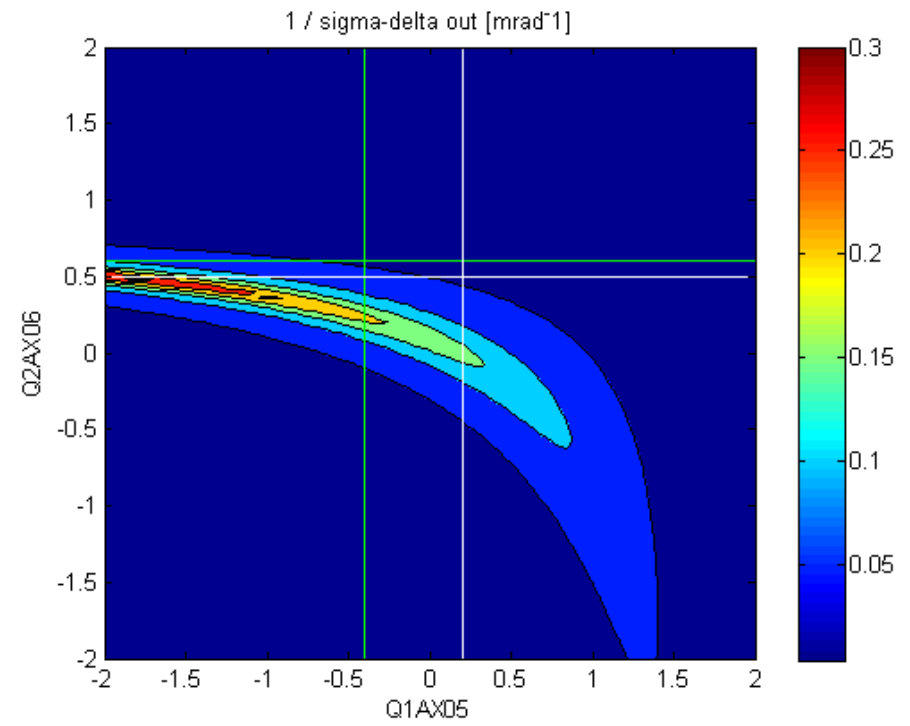

Figure 7.8: T3D MatLab Calculated $\sigma_{z}$ Q1AX05-Q2AX06 quadrupole scan, plotting $1 / \sigma_{\delta}$ to contrast the small energy spreads. The white cross hairs indicate the Feb 62009 data set operating point, while the green cross hairs indicate the Feb 112009. 
It is interesting to plot the calculated ratio of $\epsilon_{z_{\text {in }}} / \sigma_{x_{\text {out }}} \cdot \sigma_{x_{\text {out }}^{\prime}}$, Figure 7.9 , and $\epsilon_{x_{\text {in }}} / \sigma_{\delta_{\text {out }}}$. $\sigma_{z_{\text {out }}}$, Figure 7.10, which is equivalent to our measured data's presentation. These plots clearly demonstrate that $\epsilon_{x_{i n}} / \sigma_{\delta_{\text {out }}} \cdot \sigma_{z_{\text {out }}}$ is very sensitive to the choice of input operating conditions; conversely $\epsilon_{z_{\text {in }}} / \sigma_{x_{\text {out }}} \cdot \sigma_{x_{\text {out }}^{\prime}}$ is relatively insensitive. These calculations are consistent with our measured projected emittance ratios in the February 6 and 11 data sets.

Further, these calculations show that the most critical input parameter to the $\epsilon_{x_{\text {in }}} / \sigma_{\delta_{\text {out }}}$. $\sigma_{z_{o u t}}$ ratio the was $\alpha_{x_{i n}}$. Thus, the quadrupole scan calculation was repeated for several values of $\alpha_{x_{i n}}$ while holding all of the other input beam characteristics fixed, the calculated quadrupole scan was performed for six values of $\alpha_{x_{i n}}$ and are shown in Figure 7.11. February 6 and 11 data sets were both taken with $\alpha_{x_{i n}}=0$.

As a check, these calculations were repeated for the ideal emittance exchange line. Specifically, the $T M_{110}$ Mode Cavity was replaced with a perfect thin lens cavity, 3.5 with $k=-1 / D$, equally flanked by two drift lengths to otherwise maintain the exact same beamline optics described above. As was expected, the ratio's of $\epsilon_{x_{\text {in }}} / \epsilon_{z_{\text {out }}}$ and $\epsilon_{z_{\text {in }}} / \epsilon_{x_{\text {out }}}$ were 1:1 over all of the input quadrupole space. The ratio of $\epsilon_{z_{\text {in }}} / \sigma_{x_{\text {out }}} \cdot \sigma_{x_{\text {out }}^{\prime}}$ was nearly 1:1 over most of the quad space, departing at most a few percent as shown in Figure 7.12. However, calculating $\epsilon_{x_{\text {in }}} / \sigma_{\delta_{\text {out }}} \cdot \sigma_{z_{\text {out }}}$, thus neglecting the correlation terms, still showed a strong and similar region of minimum $\sigma_{z} \cdot \sigma_{\delta}$ dependant on the choice of input quadrupole settings and is shown in Figure 7.13. Thus, by only taking the minimum product projections, $\sigma_{z} \cdot \sigma_{\delta}$, and neglecting the correlation terms we may not be operating at the location that minimizes the residual coupling from the thick lens deflecting cavity. Clearly, this demonstrates that having the ability to resolve the output longitudinal correlation is critical to the measurement of direct emittance exchange. 


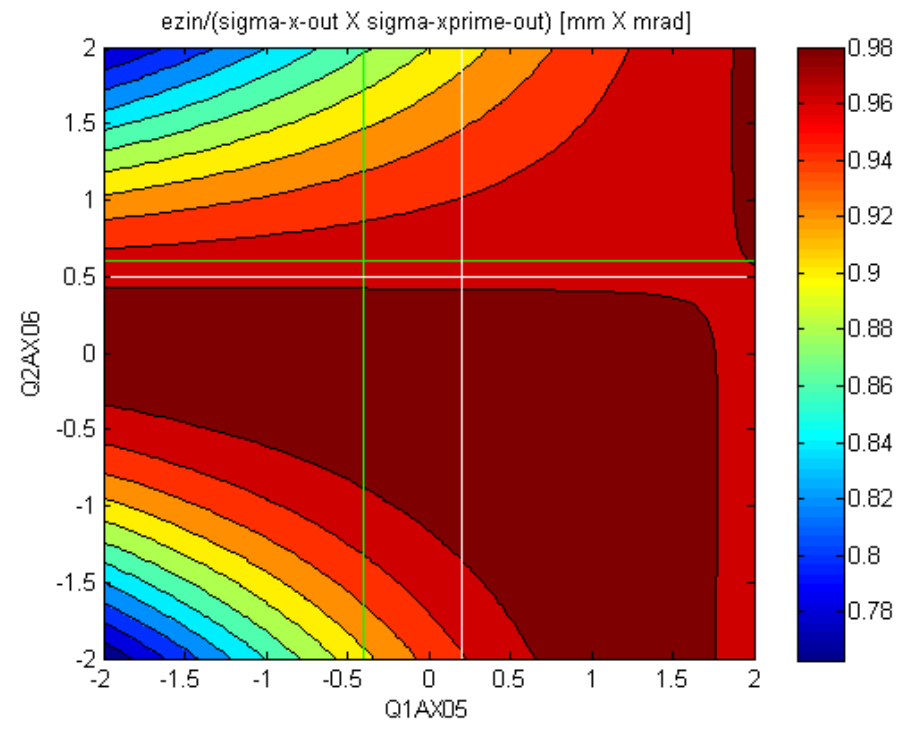

Figure 7.9: T3D MatLab Calculated Q1AX05-Q2AX06 quadrupole scan, plotting $\epsilon_{z_{\text {in }}} / \sigma_{x_{\text {out }}}$. $\sigma_{x_{\text {out }}^{\prime}}$. The white cross hairs indicate the Feb 62009 data set operating point, while the green cross hairs indicate the Feb 112009.

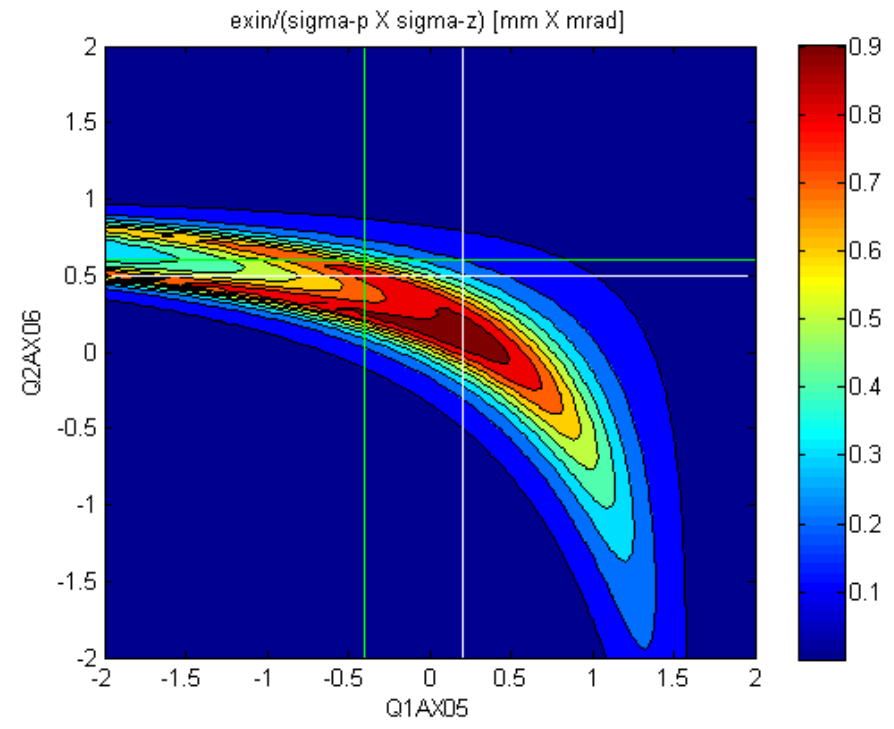

Figure 7.10: T3D MatLab Calculated Q1AX05-Q2AX06 quadrupole scan, plotting $\epsilon_{x_{\text {in }}} / \sigma_{z_{\text {out }}} \cdot \sigma_{\delta_{\text {out }}}$. The white cross hairs indicate the Feb 62009 data set operating point, while the green cross hairs indicate the Feb 112009. 

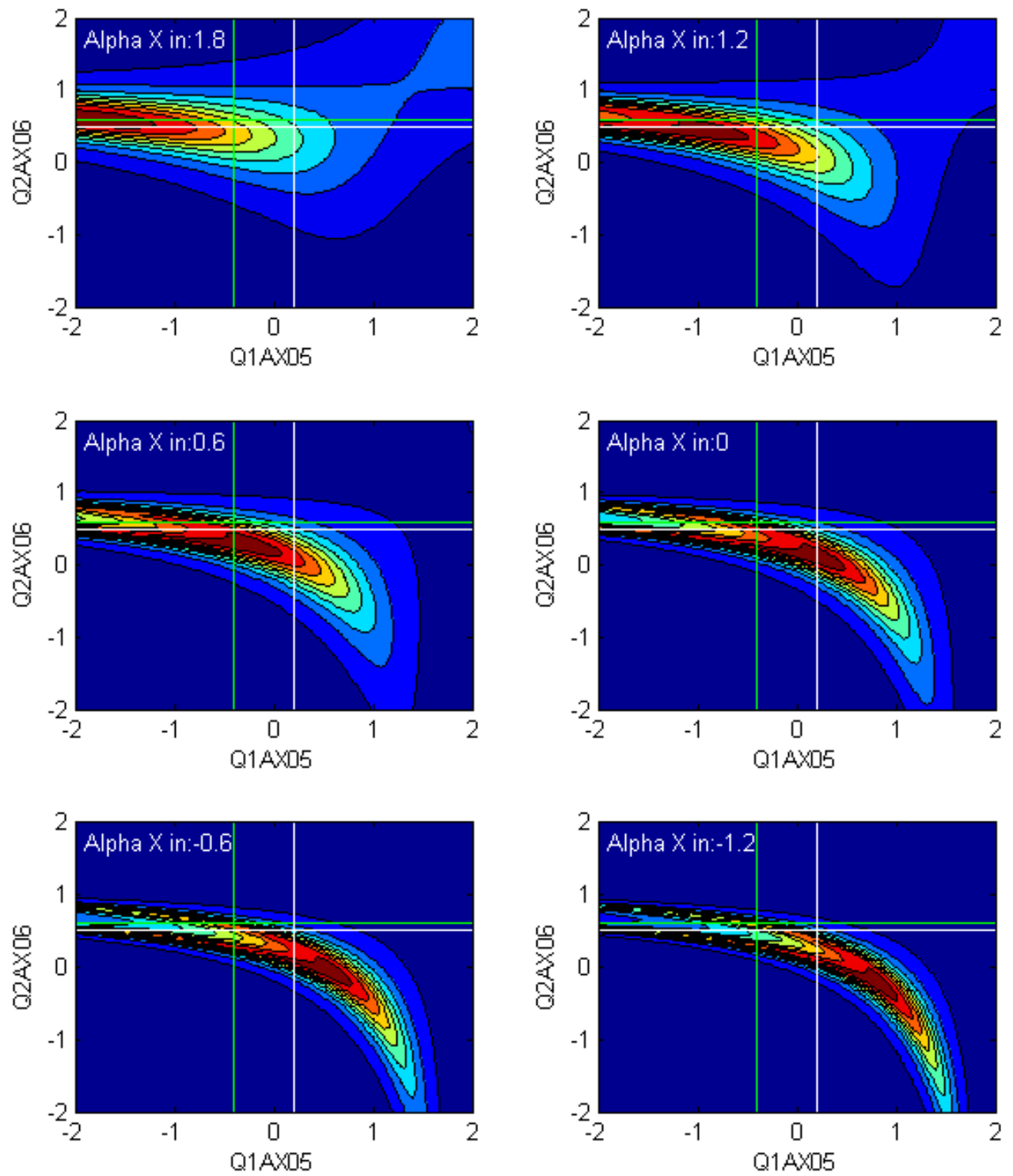

Figure 7.11: T3D MatLab Calculated Q1AX05-Q2AX06 quadrupole scan, plotting $\epsilon_{x_{\text {in }}} / \sigma_{z_{\text {out }}} \cdot \sigma_{\delta_{\text {out }}}$ for Several $\alpha_{x_{\text {in }}}$ values - Feb 6 and Feb 11 data sets were taken with $\alpha_{x_{i n}} \approx 0$. The dark red indicates the region of a 1:1 ratio. The white cross hairs indicate the Feb 62009 data set operating point, while the green cross hairs indicate the Feb 11 2009. 


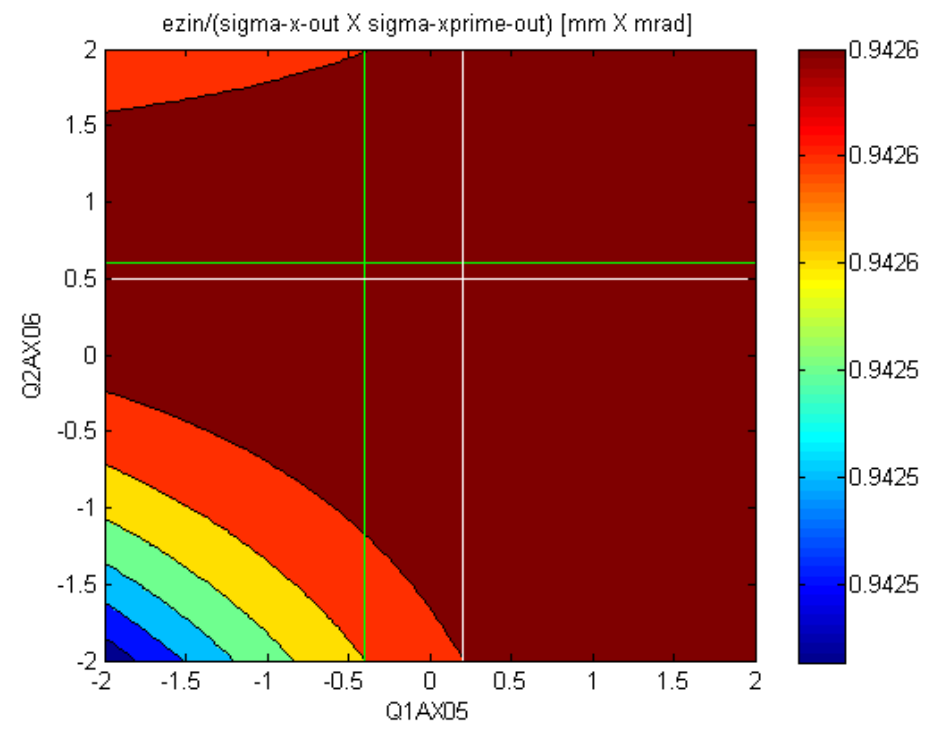

Figure 7.12: Using a thin lens cavity producing an ideal $M_{E E X}$, a T3D MatLab calculated Q1AX05-Q2AX06 quadrupole scan plotting $\epsilon_{z_{\text {in }}} / \sigma_{x_{\text {out }}} \cdot \sigma_{x_{\text {out }}^{\prime}}$. The white cross hairs indicate the Feb 62009 data set operating point, while the green cross hairs indicate the Feb 11 2009 .

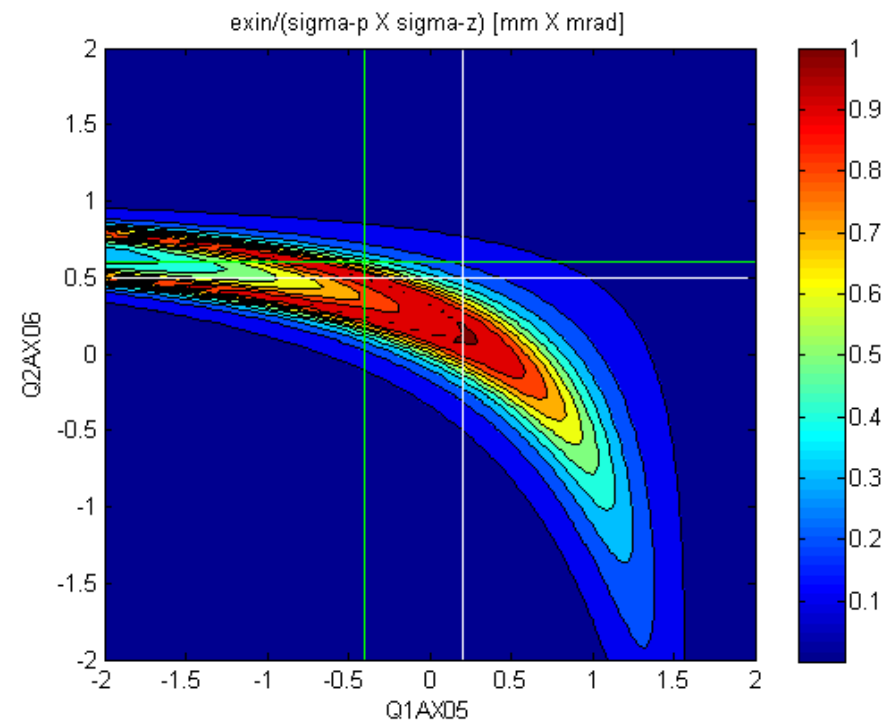

Figure 7.13: Using a thin lens cavity producing an ideal $M_{E E X}$, a T3D MatLab calculated Q1AX05-Q2AX06 quadrupole scan, plotting $\epsilon_{x_{\text {in }}} / \sigma_{z_{\text {out }}} \cdot \sigma_{\delta_{\text {out }}}$. The white cross hairs indicate the Feb 62009 data set operating point, while the green cross hairs indicate the Feb 11 2009 . 


\subsection{Conclusions}

The proof of principle for an emittance exchange has been demonstrated by observing, within uncertainties, a 1:1 projected emittance exchange as $\epsilon_{z_{i n}}$ of $21.1 \pm 1.5 \mathrm{~mm} \cdot \mathrm{mrad}$ to an $\epsilon_{x_{\text {out }}}$ of $20.8 \pm 2.00 \mathrm{~mm} \cdot \mathrm{mrad}$ and a $\epsilon_{x_{\text {in }}}$ of $4.67 \pm 0.22 \mathrm{~mm} \cdot \mathrm{mrad}$ exchanged to a $\epsilon_{z_{\text {out }}}$ of $7.06 \pm 0.43 \mathrm{~mm} \cdot \mathrm{mrad}$. Plausible explanations for the apparent projected emittance growth in the $\epsilon_{x_{i n}} \Rightarrow \epsilon_{z_{\text {out }}}$ are the effects of the non-ideal emittance exchange matrix with a nonzero $R_{4,3}$ value, the choice of input operating beam characteristics, and resolution in the measurement of $\sigma_{p_{\text {out }}}$. Additionally, space charge forces and coherent synchrotron radiation, that latter of which are discussed in the final chapter, may significantly contribute to the emittance dilution.

Our reported emittance exchange observations are only at two operating locations of transverse input conditions, each with the same fixed input longitudinal beam parameters and bunch charge. Based on these initial findings, supported by calculation, an extensive program will begin to map the large parameter space. As demonstrated by calculation, there is a need for further development of the transverse and especially the longitudinal diagnostics that would allow for a direct measurement of the $z-\delta$ correlation in the bunch. 


\section{Chapter 8}

\section{Conclusions}

An experimental program to perform a proof of principle transverse to longitudinal emittance exchange has been completed at the Fermilab A0 Photoinjector. A new beamline and unique $T M_{110}$ deflecting mode RF cavity has been constructed and implemented at the Photoinjector to create the emittance exchange channel. The method of difference orbits was used to measure the beamline transport matrix. The emittance exchange beamline was measured to produce an optics transport that produced transverse to longitudinal emittance exchanger. The finite length of the $T M_{110}$ Mode Cavity resulted in a non-ideal emittance exchange transport matrix with a non-zero $R_{43}$ element. The measured $R_{43}$ element did not match expected values. The value of the $R_{23}$ element also did not agree with the expected value. These two elements require a remeasurement, especially $R_{43}$, since it holds a significant impact on the emittance dilution.

Upon verification of the emittance exchange transport matrix, an initial observation of an emittance exchange was performed. Beam parameters of a $14.3 \mathrm{MeV}$ electron beam were measured at the input and output of the emittance exchange beamline. Operating at a bunch charge of $250 \mathrm{pC}$, a 1:1 ratio of input longitudinal to output horizontal emittance, $\epsilon_{z, \text { in }} \Leftrightarrow \epsilon_{x, \text { out }}$, exchange was observed. An input-transverse to output-longitudinal emit-

tance, $\epsilon_{x, \text { in }} \Leftrightarrow \epsilon_{z, \text { out }}$, exchange was observed with projected emittance growth: $4.67 \pm 0.22$ $\mathrm{mm} \cdot \mathrm{mrad}$ exchanged to a $\epsilon_{z_{\text {out }}}$ of $7.06 \pm 0.43$. In addition to the non-ideal emittance exchange matrix, i.e. effects of the thick lens cavity, this dilution can be a result of choice of operating input conditions, space charge forces, and potential coherent effects and resolution of the measurements. Calculation showed that the ratio of $\epsilon_{x, \text { in }} \Leftrightarrow \epsilon_{z, \text { out }}$ is sensitive to the input conditions, while $\epsilon_{z, \text { in }} \Leftrightarrow \epsilon_{x, \text { out }}$ is not as significant.The apparent $\epsilon_{z_{\text {out }}}$ growth is 
consistent with calculated values in which the correlation term is neglected.

\subsection{Emittance Exchange Future}

The following subsections are brief discussions of future investigations for both further understanding as well as expanding upon the installed emittance exchange program.

\subsubsection{Space Charge \& CSR Studies}

The high charge bunches encountered in our emittance exchange experiment experience strong internal space charge forces, which give rise to energy spreads along the bunch. From calculations in Section 7.6, we can expect output rms bunch lengths on order of $0.2 \mathrm{~mm}$ and bunches having momentum spreads as low as $10 \mathrm{keV}$. H. Edwards has estimated the longitudinal electric fields for two different bunch geometries, both with uniform charge densities: an ellipsoidal bunch and a cylindrical bunch, for differing transverse beam radii and longitudinal bunch lengths.[70] Both methods calculate linear space charge forces within the bunch. The ellipsoidal bunch calculation follows the Lapostolle space charge treatment,[71] which is form used in Trace 3D's space charge calculation.

$$
E_{z}=\frac{Q}{e \pi \varepsilon_{o}} \frac{3 f}{r_{x} r_{y} r_{z}} z
$$

where $f$ is form factor that varies from 0 to 1 dependant on $p=\gamma r_{z} / \sqrt{r_{x} r_{y}}$

The estimate with a cylindrical bunch shape utilized Ferrario's HOMDYN calculation gives similar $E_{z}$ values.[72] From these calculations, an approximation of the energy spread due to the space charge can be made after conversion to an rms size is made. Figure 8.1. As shown in Tables 7.1 and 7.2 experimental measurements at X24 have yielded output $\sigma_{z}$ on order of $0.24 \mathrm{~mm}$. The geometric average of the transverse spot sizes are on the order of $1.4 \mathrm{~mm}$. Thus from Figure 8.1 we can anticipate an rms longitudinal electric field of $18 \mathrm{kV} / \mathrm{m}$. Since the energy spread measurement occurs 1.4 meters downstream, we might anticipate energy spreads on order $25 \mathrm{keV}$ from space charge alone. However, in actuality we measure smaller energy spreads, on the order of $20 \mathrm{keV}$. Thus there seems to be a slight overestimation. The second calculation performed by H. Edwards approximated the bunch 


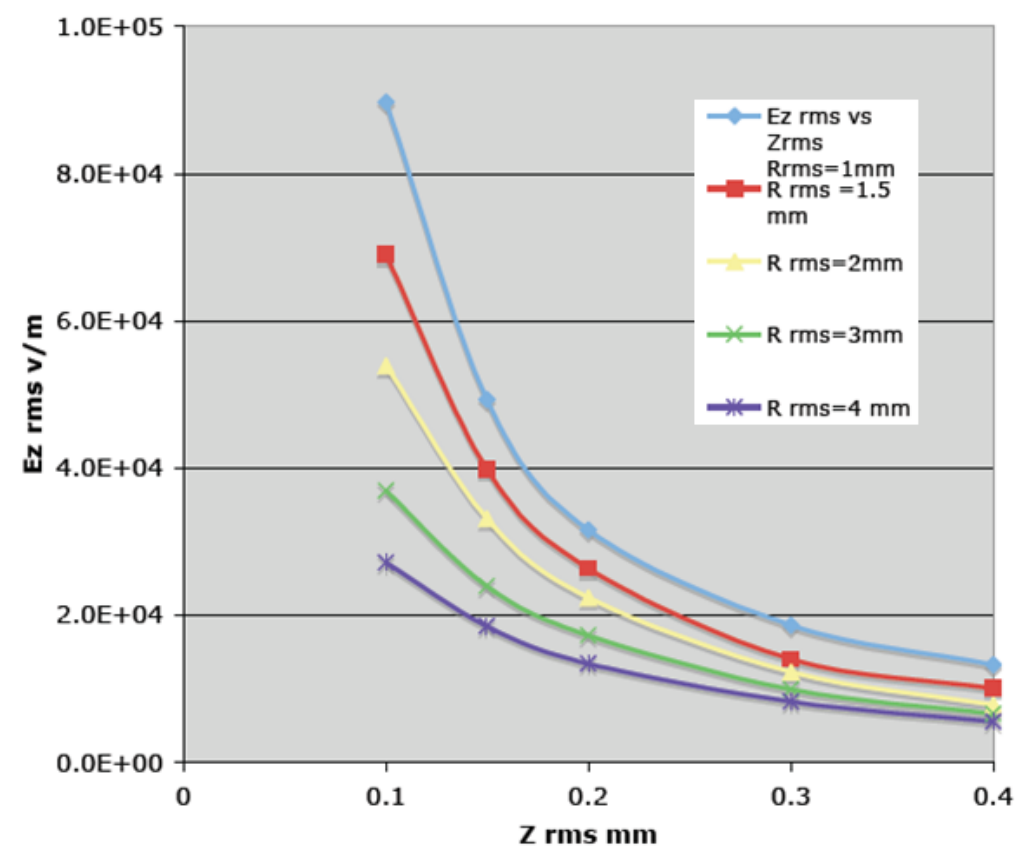

Figure 8.1: Longitudinal space charge field calculation for a $250 \mathrm{pC}$ ellipsoidal bunch of unform charge density.

as a cylinder and likewise returned a result of an $\mathrm{rms} E_{z}$ of $18 \mathrm{kV} / \mathrm{m}$.

A similar space charge calculation was performed by Yin-E Sun using the code GPT.[6]. The preliminary GPT results returned a value of approximately $6 \mathrm{kV} / \mathrm{m}$ for, thus suggesting an energy spread of $10 \mathrm{keV}$ due to space charge - smaller than our measured quantities.

To better benchmark the space charge calculation the emittance exchange experiment needs to be performed with varying bunch charge, i.e. $500 \mathrm{pC}$ and at $1 \mathrm{nC}$ if possible. Additionally, the experiment needs to be performed at several input operating conditions at the bunch charge of $250 \mathrm{pC}$.

Synchrotron radiation wavelengths on the order of the electron bunch length, thus called coherent synchrotron radiation (CSR) may introduce head-tail interaction in the bunch through dispersive sections. Simply stated, during a $|v| \approx c$ electron bunches passage through a bending magnet, CSR emitted from the tail of the bunch, traveling along the chord of the bunches arc, can catch up to the head of the bunch. The interaction of the CSR fields along the bunch can deleteriously effect the energy spread. The A0 Photoinjector group will be attempting to estimating the impact CSR may have. 


\subsubsection{Spatial to Temporal Modulation}

Manipulation of the transverse-to-longitudinal phase space may be able to produce ultrashort bunch trains, $\approx 55 \mathrm{fs}$ rms bunches spaced 330 fs apart.[74] Such bunch trains may open possibilities for future light sources. In the proposed experiment by Y. Sun, the input bunch is transversely modulated by destructively intercepting the beam with a mask - akin to the slits used for the transverse emittance measurements. Through the action of the emittance exchanger, the horizontal spatial modulation can be transferred to a longitudinal (temporal) modulation. By referring to the matrix, Equation 3.9, for the thin lens emittance exchange:

$$
M_{E E X}\left(k=k_{o}\right)=\left(\begin{array}{cccc}
0 & 0 & -\frac{1}{\alpha}-\frac{l}{D} & -\alpha l \\
0 & 0 & -\frac{1}{D} & -\alpha \\
-\alpha & -\alpha l & 0 & 0 \\
-\frac{1}{D} & -\frac{1}{\alpha}-\frac{l}{D} & 0 & 0
\end{array}\right)
$$

we can relate the output and input beam parameters as:

$$
\left(\begin{array}{c}
x \\
x^{\prime} \\
z \\
\delta
\end{array}\right)_{\text {out }}=M_{E E X}\left(\begin{array}{c}
x \\
x^{\prime} \\
z \\
\delta
\end{array}\right)_{i n}=\left(\begin{array}{c}
-z_{i n} \frac{1}{\alpha}-z_{i n} \frac{l}{D}-\delta_{i n} \alpha l \\
-\frac{1}{D} z_{i n}-\delta_{i n} \alpha \\
-x_{i n} \alpha-x_{i n}^{\prime} \alpha l \\
-x_{i n} \frac{1}{D}-x_{i n}^{\prime} \frac{1}{\alpha}-x_{i n}^{\prime} \frac{l}{D}
\end{array}\right)
$$

thereby converting the input horizontal to the longitudinal output. To preserve the horizontal bunch structure in the longitudinal output the contribution from $x_{i n}^{\prime}$ needs to be minimized. This can be achieve by proper setup of the input beam, outlined in [74]. Figure 8.2 display a simulation of the proposed experiments by Y.-E Sun and P. Piot demonstrating the spatial to temporal bunch conversion.

\subsection{3 'Poor Man's' Deflector}

A proposed experiment, reported by P. Emma and O. Shevchenko at the 2008 Workshop of X-Ray FELs, utilizes a skew quadrupole inserted in the mid section of a horizontal 

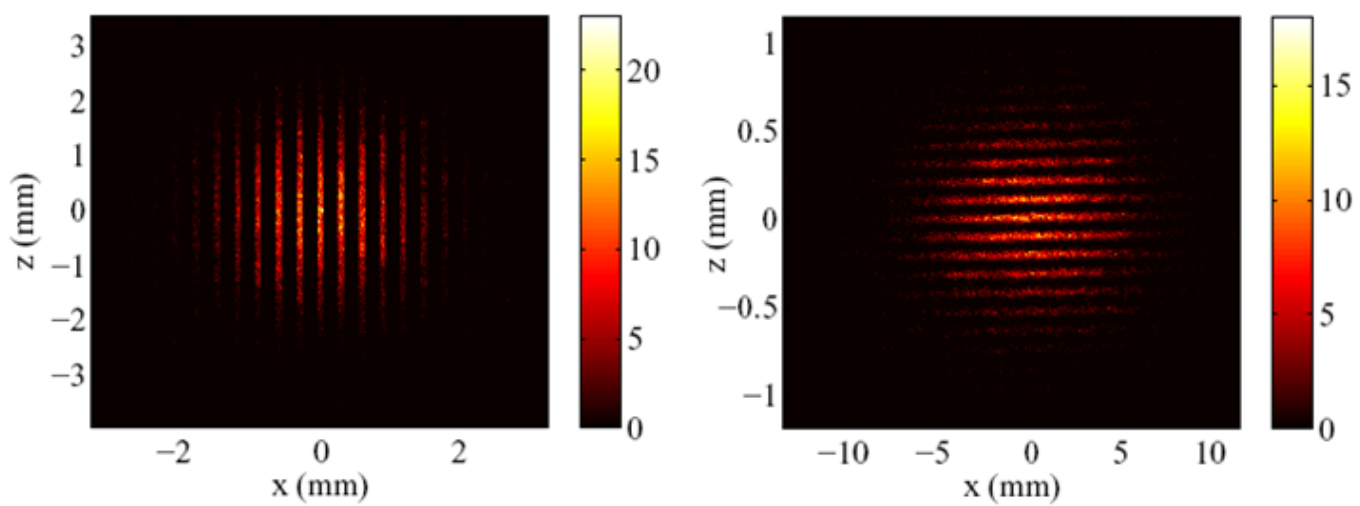

Figure 8.2: Simulations run by Y.-E Sun and P. Piot demonstrating conversion of spatial modulation to temporal modulation using the emittance exchange beamline. The left image shows the $x-z$ beam projection prior to the EEX line, while the right shows the $x-z$ projection after.

deflecting magnetic chicane.[75] As a result, the skew quadrupole couples the horizontal and vertical coordinates, imparting a vertical-time correlation. This comes about by injecting a beam of known chirp into the chicane, from which the electron bunch's time-position correlation can be ascertained from the bunch's horizontal position-momentum correlation. The skew quadrupole couples the time-position correlation into the non-dispersive vertical plane, thereby preserving the correlation in the vertical through the remainder of the horizontal magnetic chicane. Deleterious effects such as space charge and coherent synchrotron radiation can longitudinally (therefore temporally) modulate the bunch energy. Changes in momentum along the bunch are then revealed in a $x-y$ projection of the bunch. A longitudinal-time correlation can also be imparted by utilizing an expensive deflecting $T M_{110}$ Mode Cavity system, which will be discussed shortly.

With a simple modification to our EEX beamline, a skew quadrupole can be inserted immediately after the $T M_{110}$ cavity, enabling investigations into space charge and CSR effects.

\subsection{A $T M_{110}$ Mode Cavity for Bunch Length Measurement}

Finally, we will discuss the application of a 'deflecting' $T M_{110}$ cavity to the measurement of bunch lengths beyond the streak camera or interferometer's capabilities. The technique has enjoyed great success at DESY through use of a Stanford Linear Accelerator Center's 

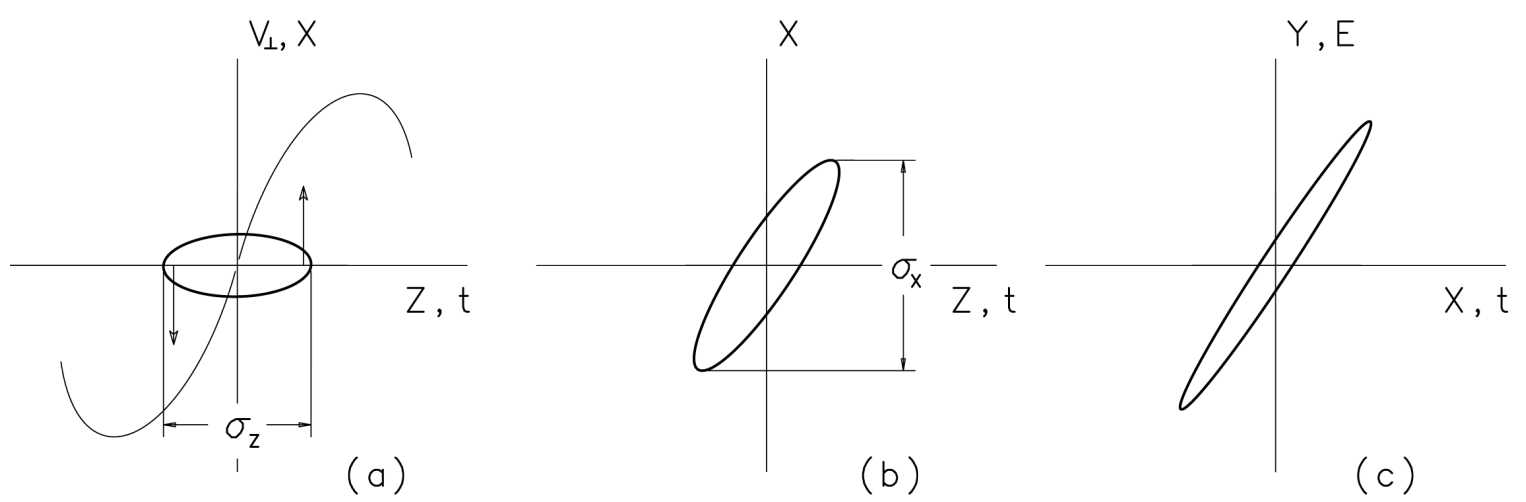

Figure 8.3: Concept for using a $T M_{110}$ Mode Cavity to impart a $z$-position dependent transverse kick to a short bunch, 'smearing' in the horizontal coordinate to obtain a longitudinal bunch length measurement.

'LOLA' cavity.[73] Further, using a $T M_{110}$ Mode Cavity in conjunction with a static momentum spectrometer magnet, a time-energy (longitudinal position-energy) correlation can be obtained. The bunch length measurement is performed at the same $T M_{110}$ Mode Cavity phase as in the emittance exchange experiment, that is the synchronous particle crosses the center of the cavity, defined as $z=0$ at $t=0$. Consequently, as depicted in Figure 8.3, the head of the electron bunch length will arrive before the vertical magnetic field has gone through zero and will undergo a horizonal deflection. The synchronous particle goes through un-deflected, and finally the tail of the bunch experiences a horizontal kick in the opposite direction. After a drift of length $l$, the beam becomes 'smeared.' The beam is transversely profiled, now having a horizontal profile to time-of-arrival correlation. We assume that the transverse kick is small compared to $\left|v_{s}\right|$ and that the bunch length is small compared to the RF wavelength $\sigma_{z} \ll \lambda_{R F}$. The following utilizes equation Equation 3.4 repeated here:

$$
\sigma_{x^{\prime}} \approx k \sigma_{z}
$$

After a drift of length $l$ the transverse spot is smeared out, the contribution to the horizontal spot size from the bunch length is $\sigma_{x_{\text {defl }}}$ :

$$
\sigma_{x_{d e f l}}=l \sigma_{x^{\prime}} \approx l k \sigma_{z}
$$

Although it is not necessary to utilize our specific $T M_{110}$ Mode Cavity described in 
Chapter 5 , it is interesting to calculate the bunch length resolution obtainable from deflection by our cavity. We take the cavity to be operating at the emittance exchange strength, $k=-1 / D$ where $\mathrm{D}$ is 0.33 meters. For reasonable Photoinjector numbers, we image the beam after a drift length $l$ of 2 meters. Assuming we can resolve a $\sigma_{x_{\text {defl }}}$ of $1 \mathrm{~mm}$, we can then resolve a bunch length of $0.16 \mathrm{~mm}$, already exceeding the streak camera and on par with the interferometer. It is certainly feasible to construct a $T M_{110}$ Mode Cavity of greater deflecting field through the use of superconducting materials.

If a spectrometer magnet is installed after the deflecting cavity, bunch length, momentum spread, and the momentum- $z,(t)$ correlation all can be obtained. To maximize the isolation between the bunch length and momentum measurements, the spectrometer is rotated to deflect $90^{\circ}$ with respect to the cavity's deflection, e.g. in the final transverse beam projection, the horizontal location would relate to time, while the vertical location would correlate momentum. Thus linear time momentum chirp would appear as a diagonal transverse beam image. 


\section{Appendix A Beam Line Element Locations}

\section{A.1 Longitudinal Element Positions w.r.t the Photocathode}

The following are tables of the longitudinal position, $s$, of pertinent beamline elements in the Straight Ahead and EEX beamlines. The detailed mechanical drawings are archived at Fermilab as drawing number 2782.000-MD-345400.

The beamline element naming convention is intended to descriptively identify the device and its approximate location. For example, Q1AX03 is the first (1) Quadrupole After Cross (X) 03.

The photo cathode is located at $s=0$. There are two types of quadrupoles encountered, denoted by their color, and mainly differ in their strength as a function of energizing current.

For a given energizing current, $i[A]$, strength of the green quadrupoles are $0.778\left[\frac{T}{m \cdot A}\right]$ while the blue quadrupoles are $0.461\left[\frac{T}{m \cdot A}\right]$.

The most critical eye will find that BPM9 and BPM10 appear in table A.2, but not table A.1, as those two BPM occur in an unused portion of the straight ahead line following the spectrometer magnet.

Only two of the correctors (trim) dipoles are listed as they are directly used in the measurement of the EEX matrix, however, there are many correctors located throughout the beamlines which are utilized for minute transverse beam corrections.

Note: the soft edge approximation extends the field of magnet a distance one half of the gap height away from the edge of the pole tip's physical (hard) edge. 


\begin{tabular}{|c|c|c|}
\hline ELEMENT & $s$ Position [mm] & Notes \\
\hline Photocathode & 0 & \\
\hline BPM1 & 305 & not shared \\
\hline H\&VTA9C & 3662 & H \& V Trim dipole \\
\hline H\&VTBX03 & 3706 & H \& V Trim dipole \\
\hline X3 & 3765 & OTR \& slits \\
\hline Q1AX03 & 3893 & Type: blue \\
\hline BPM2 & 3956 & not shared \\
\hline $\mathrm{X} 4$ & 4149 & pneumatic OTR \\
\hline $\mathrm{X} 5$ & 4563 & pneumatic YAG \\
\hline Q1AX05 & 4739 & Type: blue \\
\hline $\mathrm{X} 6$ & 5059 & pneumatic OTR \\
\hline Q2AX06 & 5358 & Type: green \\
\hline BPM3 & 5424 & not shared \\
\hline $\mathrm{X} 7$ & 5623 & OTR \& slits \\
\hline $\mathrm{X} 8$ & 7927 & YAG \\
\hline Q1AX08 & 8061 & Type: green \\
\hline Q2AX08 & 8210 & Type: green \\
\hline BPM4 & 8292 & shared with BPM24 \\
\hline Q3AX08 & 8359 & Type: green \\
\hline $\mathrm{X} 9$ & 8492 & pneumatic OTR, streak camera \\
\hline $\mathrm{X} 10$ & 8754 & pneumatic OTR \\
\hline $\mathrm{X} 12$ & 9131 & pneumatic OTR \\
\hline $\mathrm{X} 13$ & 9294 & pneumatic OTR \\
\hline $\mathrm{X} 14$ & 9939 & pneumatic OTR \\
\hline Q1AX14 & 10065 & Type: green \\
\hline Q2AX14 & 10230 & Type: green \\
\hline BPM6 & 10304 & shared with BPM25 \\
\hline Q3AX14 & 10396 & Type: green \\
\hline $\mathrm{X} 15$ & 10504 & pneumatic OTR \\
\hline BPM7 & 10786 & shared with BPM26 \\
\hline Spectrometer input edge & 11036 & soft edge value \\
\hline Spectrometer output edge & 11327 & soft edge value \\
\hline BPM8 & 11468 & Disp. $240 \mathrm{~mm}$, shared with BPM30 \\
\hline XS3 & 11574 & YAG, Dispersion $324 \mathrm{~mm}$ \\
\hline
\end{tabular}

Table A.1: Longitudinal position of beamline elements in the straight ahead beamline. 


\begin{tabular}{|c|c|c|}
\hline ELEMENT & $s$ Position [mm] & Notes \\
\hline Photocathode & 0 & \\
\hline BPM1 & 305 & not shared \\
\hline H\&VTA9C & 3662 & H \& V Trim dipole \\
\hline H/VTBX03 & 3706 & 'start of EEX line' \\
\hline X3 & 3765 & OTR \& slits \\
\hline Q1AX03 & 3893 & Type: blue \\
\hline BPM2 & 3956 & not shared \\
\hline $\mathrm{X} 4$ & 4149 & pneumatic OTR \\
\hline $\mathrm{X} 5$ & 4563 & pneumatic YAG \\
\hline Q1AX05 & 4739 & Type: blue \\
\hline $\mathrm{X} 6$ & 5059 & pneumatic OTR \\
\hline Q2AX06 & 5358 & Type: green \\
\hline BPM3 & 5424 & not shared \\
\hline $\mathrm{X} 7$ & 5623 & OTR \& slits \\
\hline D1 input edge & 5828 & soft edge value \\
\hline D1 output edge & 6121 & soft edge value \\
\hline BPM24 & 6445 & shared with BPM4 \\
\hline D2 input edge & 6694 & soft edge value \\
\hline D2 output edge & 6986 & soft edge value \\
\hline BPM25 & 7081 & shared with BPM6 \\
\hline $\mathrm{X} 21$ & 7195 & OTR \& YAG \\
\hline$T M_{110}$ & 7602 & center of mid cell \\
\hline $\mathrm{X} 22$ & 8007 & OTR \& YAG \\
\hline BPM26 & 8122 & shared with BPM7 \\
\hline D3 input edge & 8217 & soft edge value \\
\hline D3 output edge & 8509 & soft edge value \\
\hline BPM27 & 8758 & shared with BPM10 \\
\hline D4 input edge & 9082 & soft edge value \\
\hline D4 output edge & 9374 & soft edge value \\
\hline $\mathrm{X} 23$ & 9582 & OTR \& slits \\
\hline Q1AX23 & 9713 & Type: blue \\
\hline BPM28 & 9782 & shared with BPM9 \\
\hline Q2AX23 & 9852 & Type: blue \\
\hline Q3AX23 & 9993 & Type: blue \\
\hline $\mathrm{X} 24$ & 10147 & OTR \& YAG, streak camera \\
\hline BPM29 & 10444 & shared with BPM5 \\
\hline EEX Spectrometer input edge & 10546 & soft edge value \\
\hline EEX Spectrometer output edge & 10836 & soft edge value \\
\hline BPM30 & 11740 & shared with BPM30, Disp. $865 \mathrm{~mm}$ \\
\hline $\mathrm{XS} 4$ & 11845 & pneumatic OTR, Disp. $944 \mathrm{~mm}$ \\
\hline
\end{tabular}

Table A.2: Longitudinal position of beamline elements in the EEX beamline. 


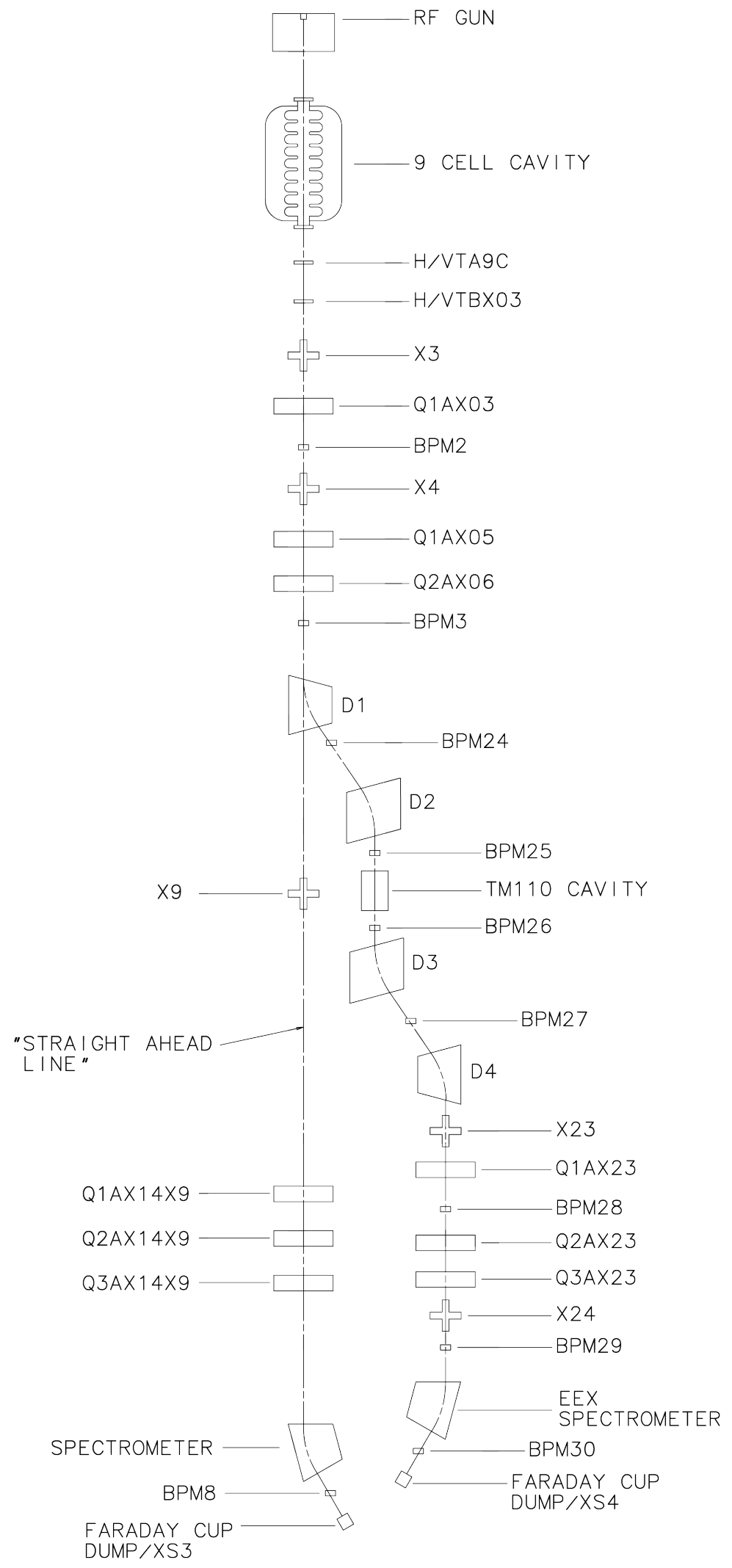

Figure A.1: This is a schematic layout of pertinent beamline components and their relative positions (Not to scale). 


\section{Appendix B Conditioning of a TESLA Input Coupler}

In 2006 a second TESLA style 1.3 GHz 9-Cell superconducting cavity was brought into operation at Fermilab, it was named Capture Cavity II, and is intended to upgrade the Photoinjector to $40 \mathrm{MeV}$. As eluded to in Section 4.1.2, the natural $Q_{o}$ of a superconducting has to be externally reduced to a more manageable $Q_{\text {loaded }} \approx 10^{6}$. This is accomplished by significantly over coupling, i.e. $\beta=Q_{o} / Q_{e x t} \approx 2000$, the input power coupler to the resonant structure resulting in a greatly reduced $Q_{\text {loaded }}$ :

$$
\frac{1}{Q_{L}}=\frac{1}{Q_{e}}+\frac{1}{Q_{o}}
$$

The required $Q_{\text {loaded }}$ is dictated by several factors. First, the required response of the control system as well as the inherent latency of the control system. Further, sensitivity to mechanical motions that the cavity will undergo during operation, either from externally induced vibrations or high gradient Lorentz Force deformations. The factors contribute to a final bandwidth that is considered practical, which in turn sets the $Q_{\text {loaded: }}$

$$
\frac{\Delta f_{F W H M}}{f} \equiv \frac{1}{Q_{\text {loaded }}}
$$

. The drawback to de-Qing a cavity is the RF power requirement to achieve a given gradient increases. For Capture Cavity II to achieve a gradient of $30 \mathrm{MV} / \mathrm{m}$ in a fill-time of $700 \mu \mathrm{s}$, an incident RF power of $200 \mathrm{~kW}$ was needed. Thus the actual hardware of the input coupler had to be able to withstand this power, and further, under conditions of $100 \%$ reflected power.

As part of the authors duties in overseeing the complete installation and commissioning 
of Capture Cavity II at Fermilab, the input coupler had to be installed and conditioned. Although the input coupler, a TESLA type III waveguide to coaxial transition, supplied with Capture Cavity II was used during the original high power horizontal test, exposure during disassembly, shipping, and reassembly prescribed re-processing. The conditioning process started by slowly introducing $\mathrm{RF}$ power under differing conditions. Initially short RF pulses of low power at a frequency away from cavity resonance. Both the pulse duration and peak RF power level were increased to the maximum of both the RF system's pulse length and power capacity.

\section{B.1 A Low Power Multipactoring Study}

Multipactoring is the term given to the multiplicative buildup of surface electron emission in RF structures. If an electron is drawn from a surface and is accelerated by a time varying electric field multifactorial (MP) can occur. If the trajectory of the electron in the $\mathrm{RF}$ electric and magnetic fields is such that it is returned to a surface with a secondary electron emission coefficient greater than 1 in an integral RF period MP will occur. Upon impact, the intial electron will launch several secondary electrons which in turn following suite. Upon their impact, the secondary electrons each generate several electrons, and so it goes. Substantial emission currents can result, drawing from the available RF power and locally delivering it to the impact sites. If enough power is available, damage to the RF component will inevitably occur. It is the goal of conditioning to identify MP behavior and to gently process away the offending electron emitters without harming the RF component

In a coaxial geometry the resonant MP state can be interrupted by the application of a DC bias. With that in mind, the design of TESLA type III input coupler incorporated a floating center conductor. The DC bias can be used either to steer clear of MP or to assist in the processesing procedure. DESY has mapped out MP behavior at relatively high RF powers, $\geq 1 \mathrm{MW}$. Capture Cavity II RF power system was limited to a maximum of 225 $\mathrm{kW}$, thus a previously unexplored power region was mapped out.

During the conditioning of Capture Caviy II's input coupler, the coupler displayed electron emission phenomena, a significant portion of which was identified as multipactoring 


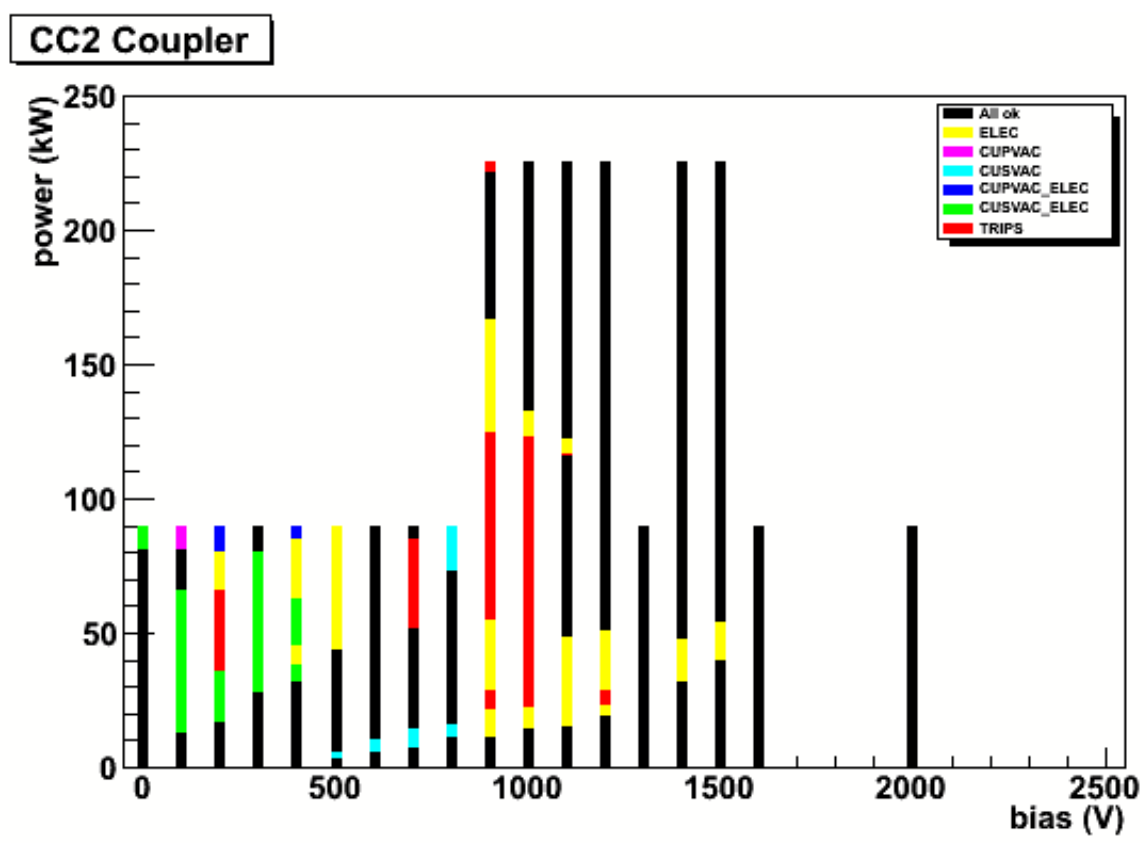

Figure B.1: The final multipactoring map of CCII Input coupler.

(MP). As expected, the MP displayed dependency on RF power and DC bias placed on the coaxial center conductor. Caution and education dictated a fully manual processing program. A retrofitted interlock system monitoring electron emission, breakdown light, temperatures, and vacuum provided the necessary fast RF inhibits during conditioning. Initially $20 \mu \mathrm{s}$ RF pulses off cavity resonance were administered at a $1 \mathrm{~Hz}$ repetition rate. The power level was increased at the rate of $1000 \mathrm{~W} / \mathrm{min}$ until maximum power was achieved, then a dwell period of 1 hour was maintained before proceeding to a longer pulse width. This continued until a pulse length of $1.4 \mathrm{~ms}$ at full RF power could be sustained without MP. Processing began with the onset of MP, at which time the RF power metered by a variable inhibit delay such that no more than 1 Joule could be delivered to the MP event. Repetitive RF power pulses narrowed the power regions where MP could be sustained. Persistent RF conditioning greatly reduced the MP but did not completely mitigate it. The operators identified that a center conductor DC bias of +1500 VDC permitted RF operation of the complete power span up to $225 \mathrm{~kW}$ with an infinite VSWR without MP. Figure B.1 shows the resulting multipactoring map of the TESLA type III in a low power region. 


\section{Appendix C $T M_{110}$ Mode Cavity Operational Notes}

This appendix is intended to serve as a guide for subsequent experimenters at the A0 Photoinjector utilizing the $T M_{110}$ Mode Cavity for beam manipulation.

Since the liquid nitrogen supply was shared with other Photoinjector R\&D efforts, even with pressure regulation, pressure fluctuations changed the tune, it was important to check the $T M_{110}$ Mode Cavity resonant frequency before applying high power RF, especially after several days of not running. The $T M_{110}$ Mode Cavity frequency measurement was accomplished in one of two ways. The easiest, was to replace the LLRF drive of ESECon with a phase stable synthesizer, such as the IFR-2042, initially set to 3.900,000,000 GHz. With the Klystron on, the synthesizer output amplitude was slowly increased until measurable $P_{f w d}, P_{r e f}$, and $P_{\text {trans }}$ diode signals were obtained on an oscilloscope, for instance Figure 5.7. The synthesizer drive frequency was then tuned for a minimum reflected power - the location of resonance - the drive frequency was then noted. This was an iterative processes, as the drive power was increased as resonance was neared.

The second method of measuring the resonant frequency was the more standard $S_{21}$ measurement, previously discussed, performed with a network analyzer. This was more time consuming as waveguide needed to be disconnected.

The tune of the superconducting 9-cell cavity dictated the overall facility's operating frequencies; typically the 9-Cell is resonant at $1.3 \mathrm{GHz}$, with fluctuates over long times of $\pm 300 \mathrm{~Hz}$. The $T M_{110}$ Mode Cavity should be at the $3^{\text {rd }}$ harmonic of this, however, as shown earlier the $T M_{110}$ Mode Cavity bandwidth is $80 \mathrm{KHz}$, so for all practical purposes,

it should always be centered on 3.900,000 GHz. Experience showed that excursions of up to $50 \mathrm{kHz}$ were tolerable if sufficient $\mathrm{HV}$ could be safely applied to the Klystron gun and 
the LLRF feed back was properly adjusted.

Once it was determined that the $T M_{110}$ Mode Cavity was operational, the ESECon LLRF drive signal was reconnected. Still without beam in the EEX line, the LLRF Fead Foward $(\mathrm{FF})$ only drive was enabled. The DOOCS magnitude setting was increased while monitoring the analog $P_{\text {trans }}$ trace on the control room's oscilloscope for an amplitude of 100 $\mathrm{mV}$, the value indicating the EEX $k_{o}$ gradient. Next was to set the Feed Back parameters, assuming that a previously generated save and restore file was not to be used. While only in FF, the operator adjusted the DOOCS displayed signal trace (black) to closely match the displayed program (green) trace for both the magnitude and phase. They were adjusted by using the respective 'channel gain' and 'channel' phase adjustments, refer to Figure 4.10. After good alignment, the operator would engage the Feed Back (FB) option. If the FB parameters were correctly set, the magnitude and phase displayed traces would snap to their green program traces. Both the proportional and integral gains should be adjusted for maximum stable values. If the FB parameters were not set correctly, or if gain values were too high, obvious magnitude and phase oscillations occurred, FB was disabled immediately, and the FB tune up procedure refined. It should be noted that several sufficiently large pressure changes in the $l N_{2}$ bath during the day would cause the cavity's frequency to wander outside the FB stability band, thus the FB had to be frequently reset.

Once the $T M_{110}$ Mode Cavity was properly operating with the phase and amplitude regulation (FB), the next step was to establish beam through the cavity. Both the $T M_{110}$ Mode Cavity FB and FF were disabled at the DOOCS panel so as the beam could be propagated through the EEX line free of the deflecting fields. The beam was steered such that BPM25 and BPM26 both read 1.6 $\pm 0.2 \mathrm{~mm}$ in the horizontal plane (see section 5.10), and both read $0 \pm 0.2$ in the vertical plane. The the LLRF drive was then re-enabled with FB; referring to figure 5.12, the proper EEX phase was set such that the BPM26 horizonal again read $1.6 \mathrm{~mm}$, while the zero crossing was in the negative direction with a positive increase in phase adjustment, or $\left.\frac{d x}{d \phi}\right|_{B P M 26}$ is negative, i.e. $\phi \approx 150 \mathrm{deg}$ in Figure 5.12.

In principle, at this point the emittance exchanger beam line was active. 


\section{Appendix D}

\section{Trace 3D Input File}

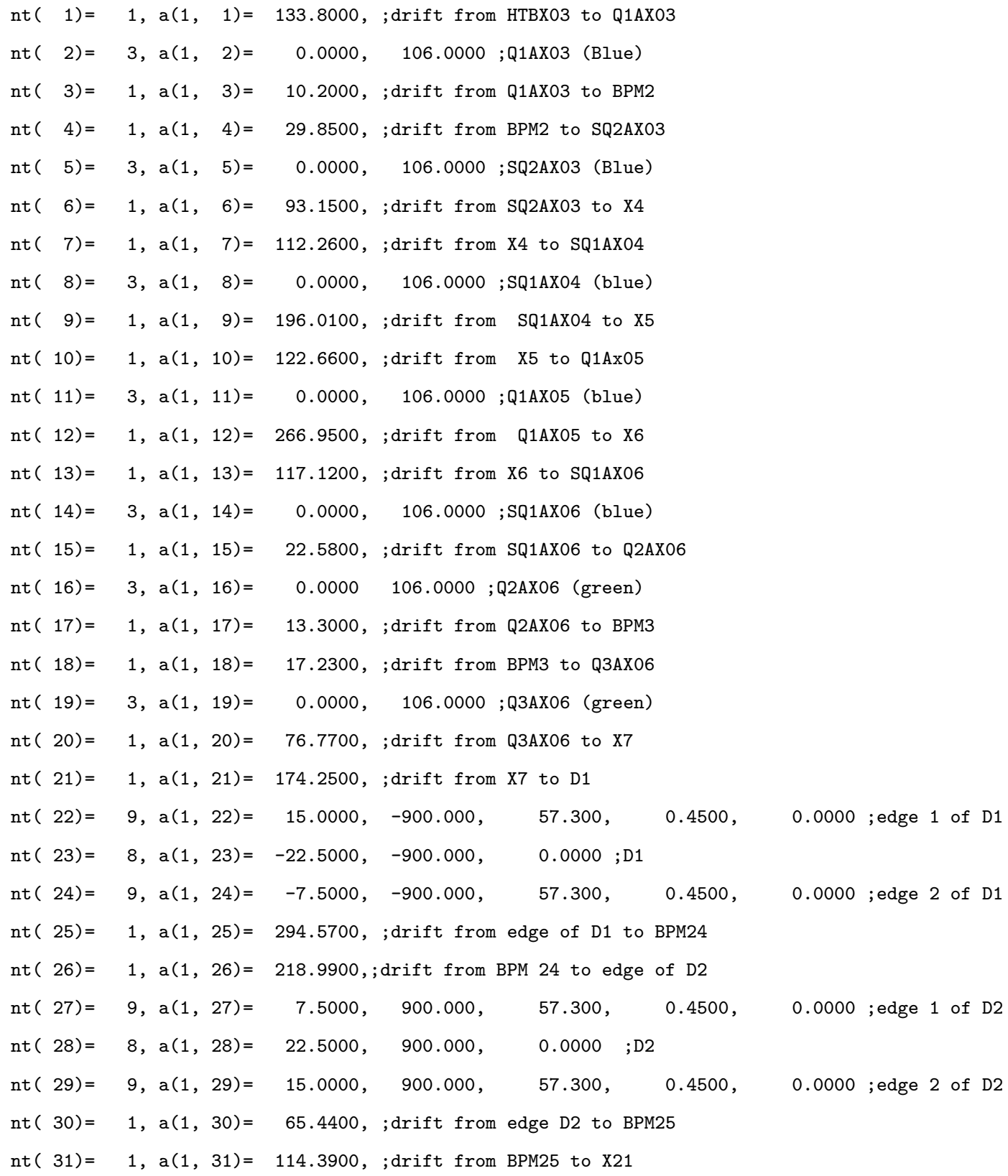




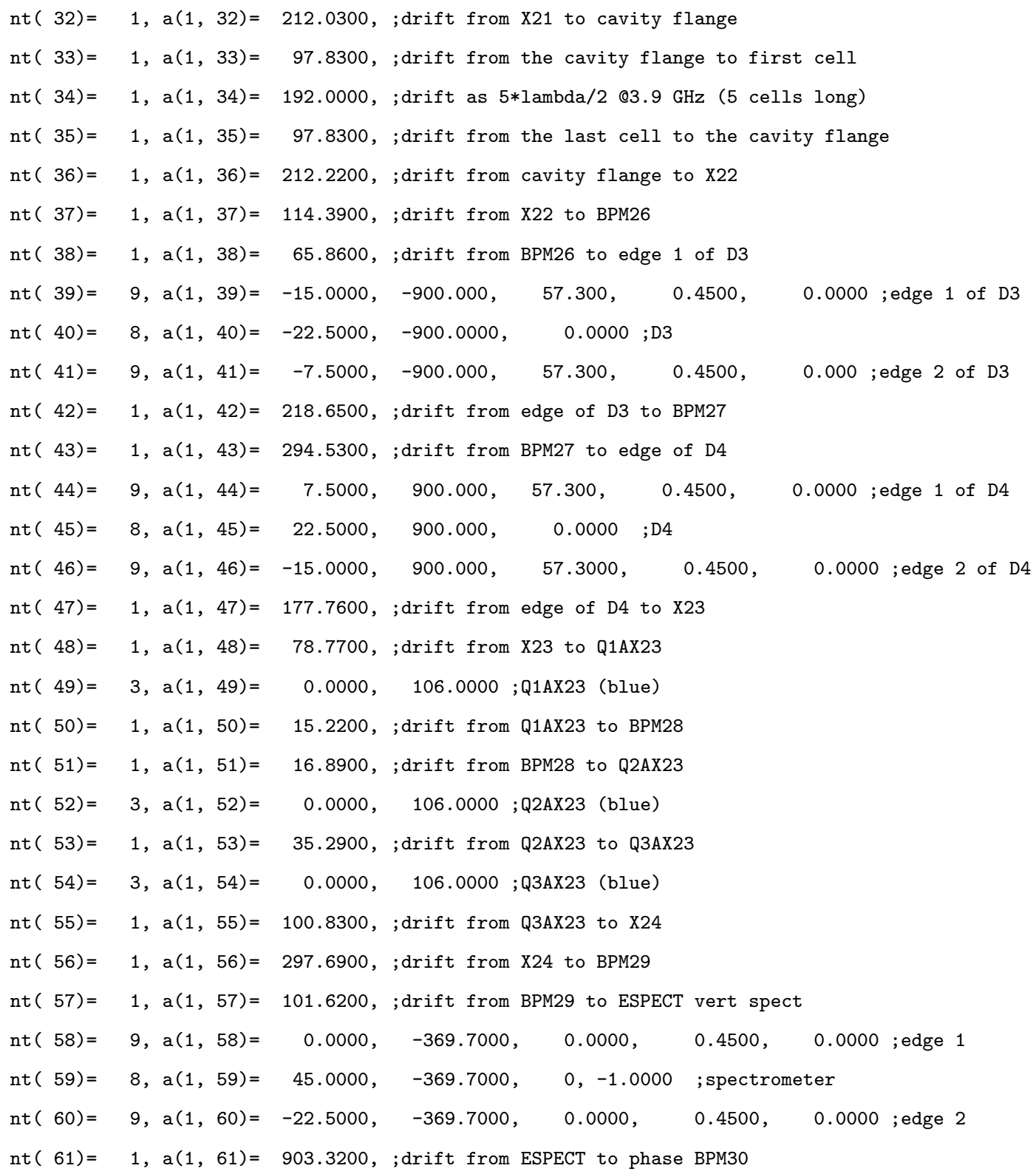




\section{Bibliography}

[1] Cornacchia, M., Emma, P., Transverse to Longitudinal Emittance Exchange Phys. Rev. Special Topics - Accelerators and Beams, Vol. 5, 084001, 2002.

[2] Kim, K.-J, Sessler, A., Transverse to Longitudinal Emittance Exchange Proceedings of the International Workshop on Beam Cooling and Related Topics, Galena IL, AIP Conf. Proc. No. 821, 2006

[3] , Kim, K.-J, Transverse-transverse and transverse-longitudinal phase-space converters for enhanced beam applications., PAC2007 Albuquerque, New Mexico, 2007

[4] Emma, P., Huang, Z., Kim, K.-J., Transverse to Longitudinal Emittance Exchange to Improve Performance of high Gain Free Electron Lasers. Phys. Rev. ST Accel. Beams Vol. 9, 100702, (2006)

[5] Edwards, D, et al. The Flat Beam Experiment at the FNAL Photoinjector, Proceedings of LINAC2000, 122, 2000

[6] Sun, Y.E. Angular-Momentum-Dominated Electron Beams and Flat-Beam Generation, PhD Thesis, University of Chicago, 1997

[7] Piot, P., Angular-Momentum-Dominated Electron Beams and Flat-Beam GenerationPhys. Rev. ST AB 9, 031001, 2006

[8] Linac Coherent Light Source Conceptual Design Report, SLAC-R-593, 2002

[9] TESLA XFEL Technical Design Report Supplement, TESLA FEL 2002-09, 2002

[10] ILC Reference Design Report, http://media.linearcollider.org/rdr-draft-v1.pdf

[11] Edwards, D. A., Syphers, M. J., An Introduction to the Physics of High Energy Accelerators Wiley, New York, 1993

[12] Lee, S.-Y., Accelerator Physics, World Scientific, River Edge, New Jersey, 1999

[13] Wille, K., The Physics of Particle Accelerators Oxford University Press, Oxford, 2000

[14] Courant, E. D., and Snyder, H. S., Theory of the Alternating Gradient Synchrotron, Annals of Physics: 3, 1-48 (1958)

[15] Fliller, R. P. General Solutions for a Transverse to Lonitudinal Emittance Exchange Beamline Fermilab Beams Document, Beams-docs-2553-V1, 2006

[16] Edwards, D. A. Notes on Transit in a Deflecting Mode Pillbox Cavity unpublished, 2007 
[17] Cornacchia, M. Emma. P Transverse to longitudinal Emittance Exchange Physics Review Special Topics - Accelerators and Beams, Vol. 5. 084001 (2002)

[18] Branlard, E., Study of a 5 Cell Copper Cavity at 3.9 GHz Unpublished, August, 2007

[19] Trace 3D, available at http://laacg1.lanl.gov/laacg/services/traceman.pdf

[20] MATLAB, http://www.mathworks.com/

[21] Fliller, R. P. Start to end simulation of transverse to longitudinal emittahce exchange at the AO Photoinjector Proceedings of European Accelerator Conference, Genoa, Italy 2008

[22] Fliller, R. P. Transverse to Longitudinal Emittance Exchange beamline at the AO Photoinjector PAC2007 Albuquerque, New Mexico, 2007

[23] ASTRA distibution website http://www.desy.de/mpyflo/

[24] Colby, E. R. Design, Construction, and Testing of a Radiofrequency Electron Photoinjector for the Next Generation Linear Collider, PhD Thesis, Univerity of California at Los Angeles, 1997

[25] Harding, D. Dttp:/tdserver1.fnal.gov/AcceleratorSupport/pbarmagnets/Magnets/NDx/NDx.html

[26] Carlsten, B.,New photoelectric injector desigh for the Los Almos Nation Laboratory XUV FEL Accelerator, NIM A285 (1998) 313-3190

[27] Aune B., et al. The superconducting TESLA cavities, Phys. Rev. ST AB 3,092001 (2000)

[28] Simrock, S. N., et al. Digital RF Control System for The DESY FLASH Linear Accelerator EUROCON, September 2007

[29] Bishopberger, S. FNPL Spectrometer Recalibration unpublished, available at http://nicadd.niu.edu/fnpl/docs/kip-spectrometer.pdf

[30] Carneiro, J.-P.et al. First Results of the Fermilab High-Brightness RF Photoinjector, in Proceedings of the 1999 Particle Accelerator Conference, A. Luccio and W. MacKay, Editors, IEEE Publishing, Piscataway, New Jersey, 1999, p. 2027-2029.

[31] Carneiro, J.-P.et al. Beam Transport, Acceleration and Compression Studies in the Fermilab High-Brightness Photoinjector, in Proceedings of the XIX International Linac Conference, ANL-98/28, Argonne National Laboratory, Argonne, Illinois, 1998, p. 878880

[32] Colestock, P., et al. Towards a Plasma-Based Wake-Field Acceleration-Based Linear Collider, Nuclear Instruments and Methods in Physics Research A 410, p. 532-543 (June 1998).

[33] Carrigan, R. A., et al.Channeling Radiation as a Probe for New Physics in the Solid State Plasma Accelerator Regime using the Fermilab A0 Photoinjector presented at the Workshop on Second Generation Laser and Plasma Accelerators (Kardamyli, Greece, June 1999) 
[34] Chao, A. W., Tigner, M., Handbook of Accelerator Physics and Engineering World Scientific,1998, pp. 191

[35] Moszynski, M.et al. Properties of the YAG:Ce scintillator Nuclear Instr. and Methods A, 345, 1994, pp.461

[36] Lumpkin, A. H., et al. Time Resolved Imaging for the APS Linac Beams Proceedings of the 1998 Linear Accelerator Confernce, 1998

[37] Tikhoplav, R. Low Emittance Electron Beam Studies PhD Thesis, Univerity of Rochester, 2006

[38] SASE FEL at TESLA Facility, Phase 2, TESLA-FEL 2002-01, 2002

[39] Piot, P. Review of experimental results of high-brightness photo-emission electron sources, in The physics and applications of high brightness electron beams, World Scientific, 2003

[40] Carneiro, J.-P. Etude Expérimentale du Photo-Injecteur de Fermilab, PhD Thesis, Université Paris XI, Orsay, France, 2001

[41] Bovet, C. et al.A selection of formulae and data useful for the design of A.G. Synchrotrons European Organization for Nuclear Research, 1970

[42] Bergoz Instrumentation, 01630 Saint Genis Pouilly, France. http://www.bergoz.com

[43] Wang, C. H., et al. Slits Measurement of Emittance on TTF Internation Conference on Accelerator and Large Experimental Physics Control Systems, Trieste, Italy, 1999

[44] Lumpkin, A. H., Ruan J., Thurman-Keup R., Notes on Streak Camera Image Processing for Bunch Length Unpublished A0 Photoinjector document

[45] Thurman-Keup, R. Bunch Length Measurement at the Fermilab AO Photoinjector Using a Marten-PupLett Interferometer, Beam Instrumentation Workshop, 2008

[46] Frolich, L., Bunch Length Measurements Using a Martin-Puplett Interferometer at the VUV-FEL PhD Thesis DESY-THESIS 2005-011, June 2005

[47] Lumpkin, A. H., Advanced, time-resolved imaging techniques for electron-beam characterizations Accelerator Instrumentation Workshop, AIP Conference Proceedings No. 229, Batavia Illinois, 1990

[48] Advanced Energy Systems, 27 Industrial Blvd., Medford NY, 11763

[49] Bellantoni, L. CKM 27 Fermilab

[50] Li, J. et al. RF Design and Processing of a Power Coupler for Third Harmonic Supeconducting Cavities Proceedings of PAC07, Alburuerque, New Mexico, 2007

[51] Hopkins, G. M. Experimental Science, Vol. 1 Munn \& Company, 1911

[52] Poisson-Superfish, available at http://laacg1.lanl.gov/laacg/services/download_sf.phtml

[53] MAFIA 3D E \& M Solver, http://www.cst.com/Content/Products/MAFIA/Overview.aspx 
[54] HFSS 3D E \& M Solver, http://www.ansoft.com/products/hf/hfss/

[55] Padamsee, H., Knobloch, J., Hays, T. RF Superconductivity for Accelerators WileyInterscience, 1998

[56] Wangler, T. Principles of RF Linear Accelerators Wiley, New York, 1998

[57] Turner, S. Editor CERN Accelerator School: Superconductivity in Particle Accelerators CERN CAS 96-03, 1995

[58] Panofsky, W.K.H., Wenzel, W.A. Some consideration concerning the transverse deflection of charge particles in radio-frequency field Rev. Sci. Inst. Vol. 27, 11 (1956), 967

[59] Edwards, D. A., Editor An RF Separated Kaon Beam from the Main Injector: Superconducting Aspects Fermilab TM-2060, 1998

[60] Koeth, T.W., A Copper 3.9 GHz TM110 Cavity for Emittance Exchange PAC2007 Albuquerque, 2007

[61] Slater, J. C. Microwave Electroncis Van Nostrand, New York, 1950

[62] Terman, F. E. Radio Engineers Handbook McGraw-Hill Book Company, New York, 1943

[63] M. McAshan and R. Wanzenberg. RF Design of a Transverse Mode Cavity for Kaon Separation Fermilab Technical TM-2144 (2001).

[64] CPI Inc., http://www.cpii.com/

[65] B. de Graff AO South Cave ODH Analysis FNAL Accelerator Division Cryogenics Document.

[66] N. Baboi, et. al Preliminary Study on HOM-Base Beam Alignment in the Tesla Test Facility MOP36 Proceeding of the LINAC 2004 Conference, Lubeck, Germany.

[67] Koeth, T. W., et al. Emittance Exchange at the Fermilab A0 Photoinjector Proceedings of European Accelerator Conference, Genoa, Italy 2008

[68] Edwards, H. T., Initial Beam Parameters for EEX at the AO Photoinjector, Unpublished

[69] Koeth, T. W., et al. Emittance Exchange at the Fermilab A0 Photoinjector Proceedings of Linear Accelerator Conference, Victoria, British Columbia, Canada, 2008

[70] Edwards, H. T., AO Photoinjector EEX Space Charge Calculations, Unpublished

[71] Lapostolle, P., CERN report AR/Int. SG/65-15, Genevea, Switzerland, 1965

[72] http://www.nicadd.niu.edu/fnpl/homdyn/manual.pdf

[73] Huening, M. et al. Observation of Femtosecond Bunch Length Using a Transverse Deflecting Structure Proceedings of FEL2005 Conference, Palo Alto, California, August 2005 
[74] Sun, Y.-E, and Piot, P., Generation of femtosecond bunch trains using a longitudinalto-transverse phase space manipulation technique, Proceedings of Linear Accelerator Conference, Victoria, British Columbia, Canada, 2008

[75] Emma, P., Shevchenko, O. A Poor Man's Tranverse Deflector Proceedings of 200 Workshop on X-Ray FEL Research and Development, LBNL, Berkeley, California, October 2008 


\section{Vita}

Timothy W. Koeth

\section{Education}

2002-2009

1993-1997

Ph.D. in Physics

Rutgers, the State University of New Jersey

New Brunswick, New Jersey

B.S. in Physics

Rutgers, the State University of New Jersey

New Brunswick, New Jersey

\section{Positions Held}

1999-2002

1998-1999

\section{Significant Publications}

- T. Czarski, W. Koprek, K. T. Pozniak, R. S. Romaniuk, S. Simrock, A. Brandt, B. Chase, R. Carcagno, G. Cancelo, T. Koeth "Superconducting Cavity Driving with FPGA Controller", Nucl.Instrum.Meth.A568:854-862,2006

- X.Xin, F. Yan, T.W. Koeth, C. Joseph, J. Hu, J. Wu, J. H. Zhao "Demonstration of 4H-SiC Visible-Blind EUV and UV Detector with Large Detection Area", Electronic Letters, Vol. 41, No. 21, October 2005 